Aus der Klinik für

Psychosomatische Medizin und Psychotherapie

(Prof. Dr. med. Ch. Herrmann-Lingen)

im Zentrum Psychosoziale Medizin

der Medizinischen Fakultät der Universität Göttingen

\title{
Psychophysiologische Stressreagibilität bei Frauen mit posttraumatischer Belastungsstörung (PTBS) sowie der Einfluss einer ausgeprägten Borderline-Symptomatik
}

\author{
INAUGURAL-DISSERTATION \\ zur Erlangung des Doktorgrades \\ der Medizinischen Fakultät \\ der Georg-August-Universität zu Göttingen
}

vorgelegt von

Juliane Albrecht

aus Erfurt

Göttingen 2013 
Dekan:

1. Berichterstatter:

2. Berichterstatterin:
Prof. Dr. rer. nat. H. K. Kroemer

Prof. Dr. med. Ch. Herrmann-Lingen

Prof. Dr. rer. nat. A. Rodenbeck

Tag der mündlichen Prüfung: 22.01.2014 


\section{Inhaltsverzeichnis}

1

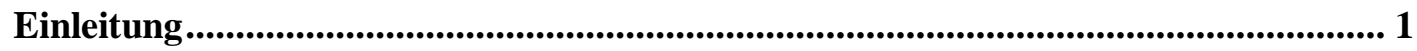

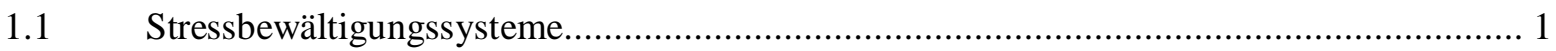

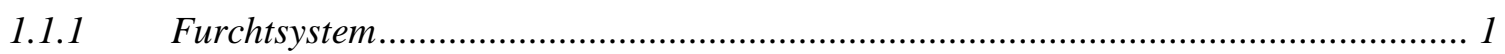

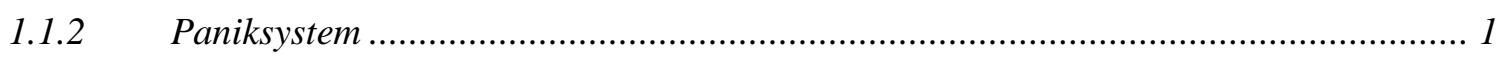

1.1.3 Erstarrungssystem und Dissoziation ...................................................................... 2

1.2 Das autonome Nervensystem und sympathovagale Dysbalance ........................................ 2

1.2.1 Indizes sympathischer und vagaler Aktivität ......................................................... 3

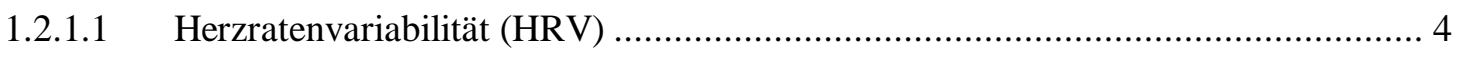

1.2.1.2 Baroreflexsensitivität (BRS) ….............................................................. 5

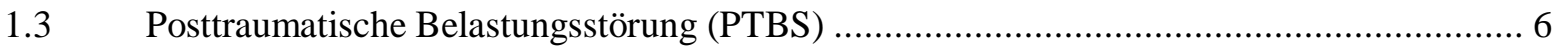

1.3.1 Klinische Merkmale der PTBS - Maladaptive Stressbewältigung............................... 6

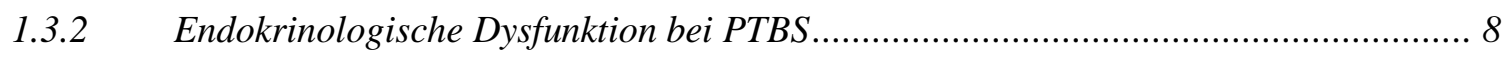

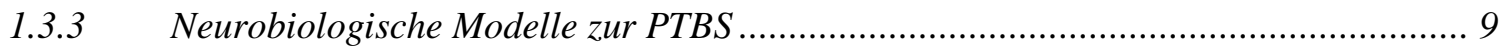

1.3.4 Gestörte physiologische Stressreagibilität bei PTBS .............................................. 9

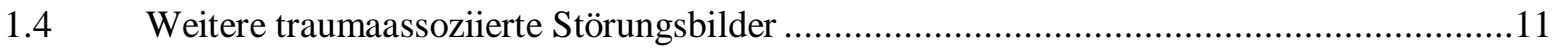

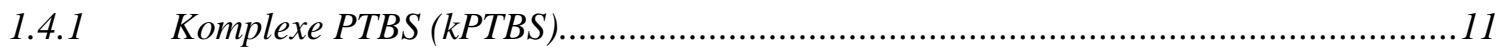

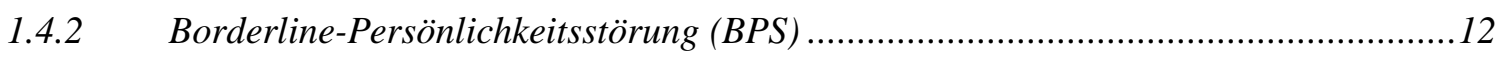

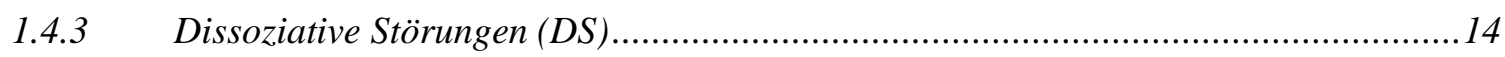

1.5 Traumazentrierte Therapie der kPTBS bzw. BPS auf der Station 9 des Asklepios

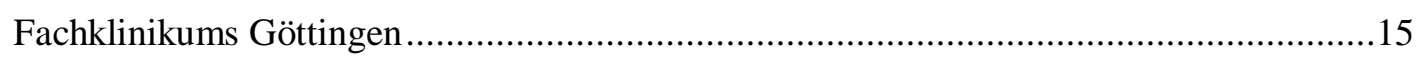

$2 \quad$ Zielsetzung und Hypothesen ........................................................................................17

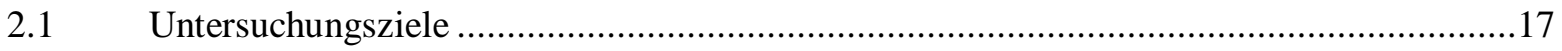

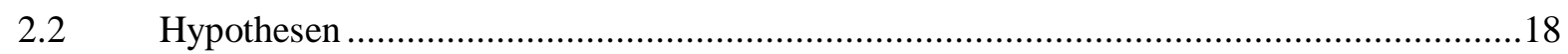

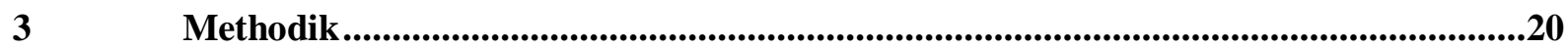

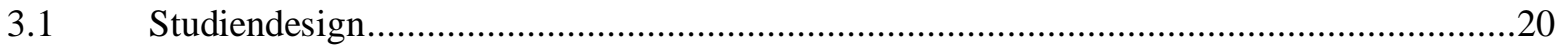

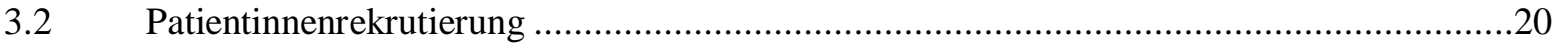

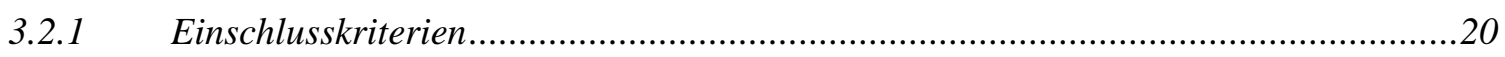

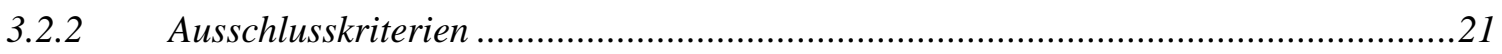

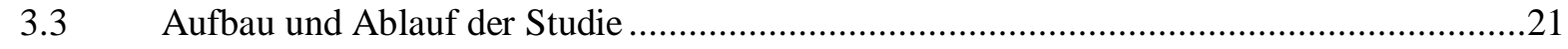

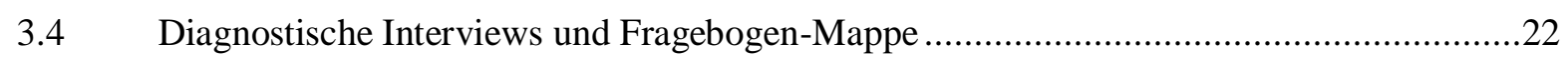

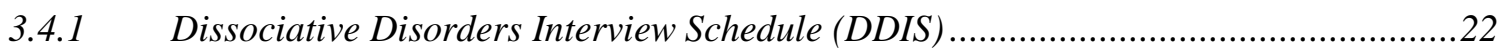

3.4.2 International Personality Disorder Examination (IPDE) .........................................23

3.4.3 Das Interview zur Diagnostik der komplexen posttraumatischen Belastungsstörung

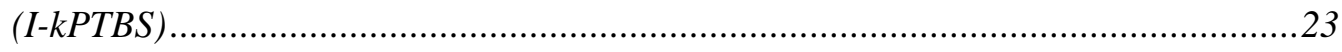

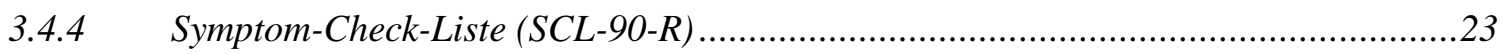

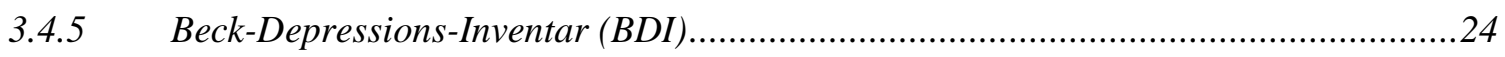




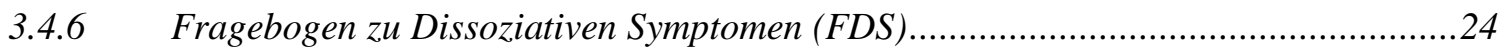

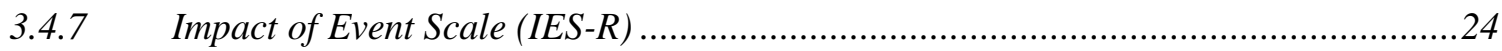

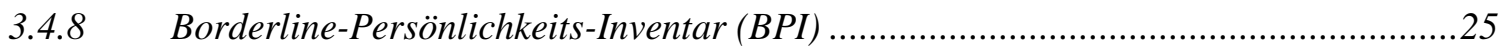

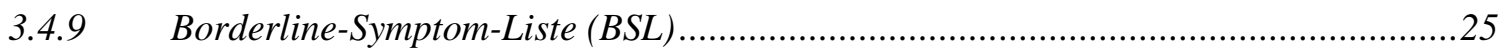

3.4.10 Allgemeiner Fragebogen zur Erfassung soziodemographischer Daten und

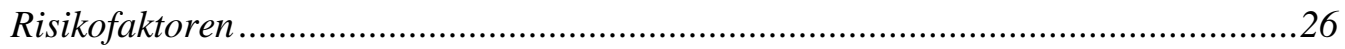

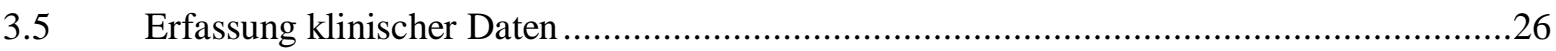

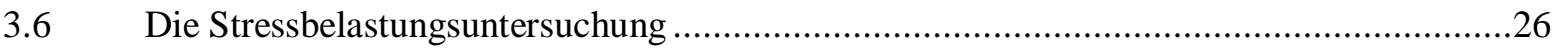

3.6.1 Die Stresstests ,,Rechnen “ und , Babyschreien “.....................................................26

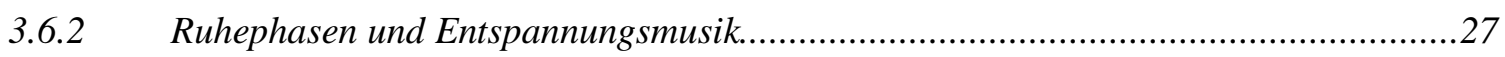

3.6.3 Analogskala subjektives Stressempfinden und beobachtbare emotionale bzw.

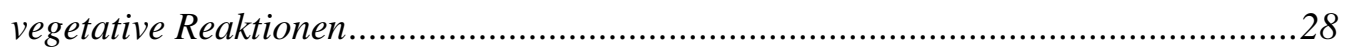

3.7 Der Task Force Monitor (Kardiovegetative Funktionsmessung) .......................................29

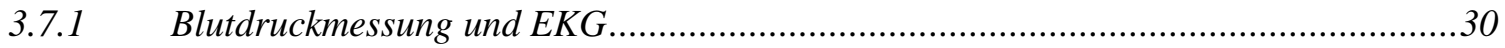

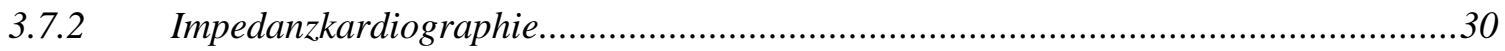

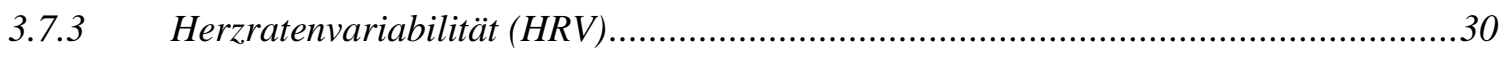

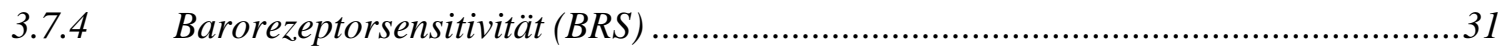

3.7.5 Auswahl der TFM-Parameter für die Auswertung der Stressreagibilität .....................31

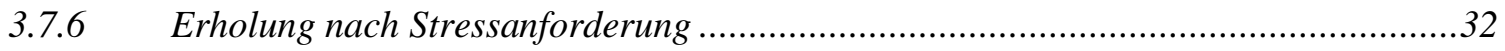

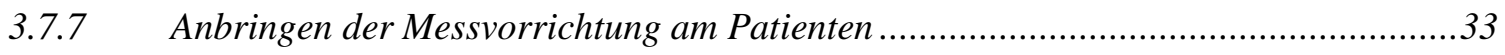

3.7.8 Auswertung und Ausschluss von Task-Force-Monitor-Daten .........................................

3.8 Bildung der Patientinnen-Subgruppe „hoch Borderline“ .................................................34

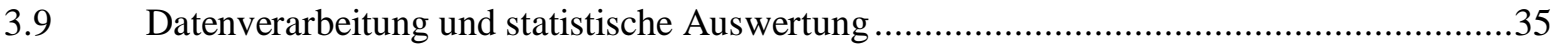

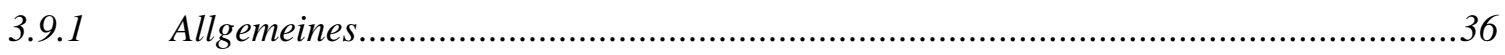

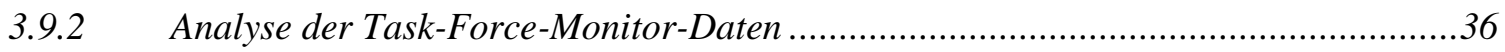

3.9.3 Auswertung der subjektiven Aktivierung (Analogskala „, Stressempfinden “ und emotionale bzw. vegetative Reaktionen)..................................................................

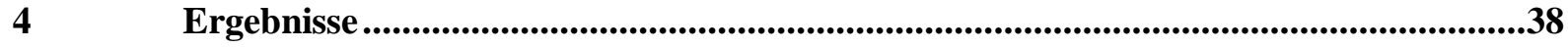

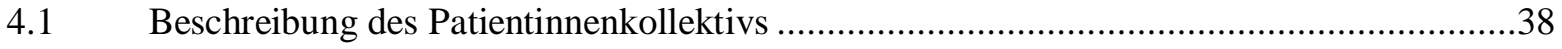

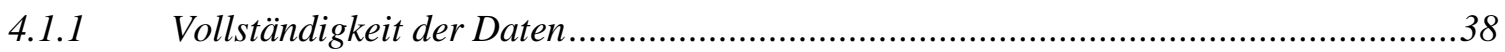

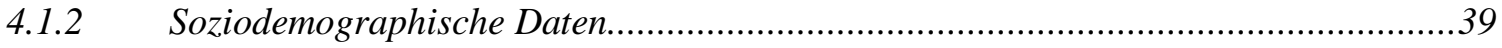

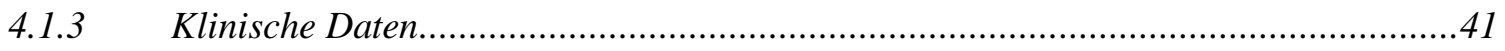

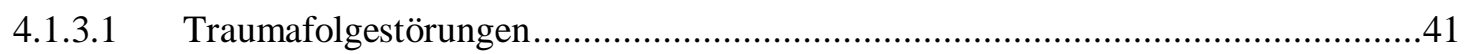

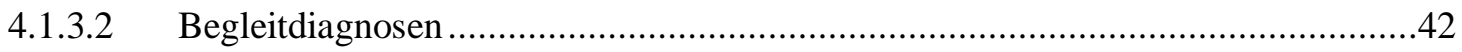

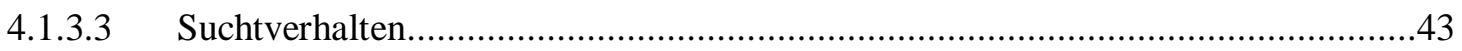

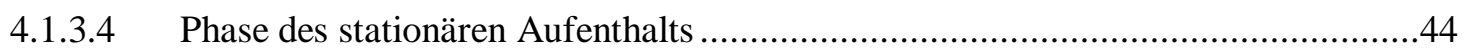

4.1.3.5 Traumaursache/Art der Traumatisierung ...........................................................4

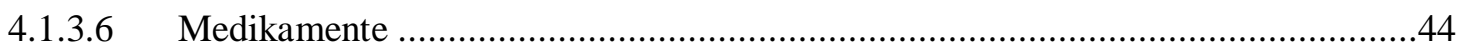

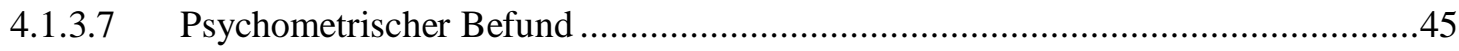

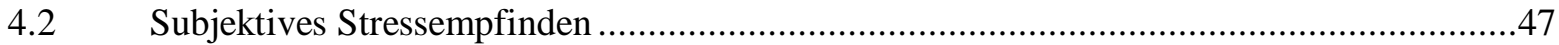


4.3 Beobachtbare emotionale bzw. vegetative Reaktionen .50

4.4 Auswertung physiologischer Stressparameter während der Stressbelastungsuntersuchung (Task-Force-Monitor-Messung)

4.4.1 Verlauf der Task-Force-Monitor-Parameter auf kollektiver Ebene..............................53

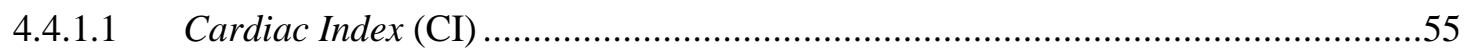

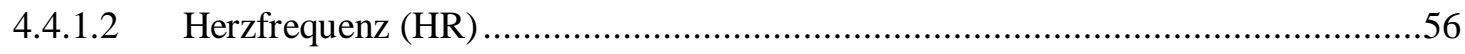

4.4.1.3 Totaler peripherer Widerstands-Index (TPRI) ….............................................57

4.4.1.4 Systolischer, diastolischer und mittlerer arterieller Blutdruck (sBP, dBP, mBP) ...58

4.4.1.5 Standardabweichung aller RR-Intervalle (SDNN) ..........................................59

4.4.1.6 Hochfrequenzband der Herzratenvariabilität (HF-HRV) ......................................59

4.4.1.7 Niederfrequenzband der Herzratenvariabilität (LF-HRV) ......................................60

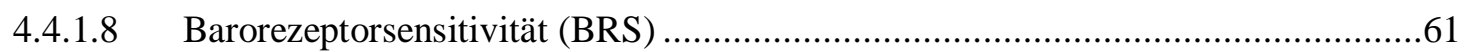

4.4.2 Vergleich der beiden Stresstests ,, Rechnen “ und ,, Babyschreien “ ...........................62

4.4.3 Erholung nach Stressanforderung , Babyschreien “ .................................................63

4.5 Patientinnen-Subgruppe ,hoch Borderline“ ......................................................................64

4.5.1 Psychometrischer Befund der Subgruppe , hoch Borderline “..................................64

4.5.2 Subjektives Stressempfinden und vegetative bzw. emotionale Reaktionen ...................66

4.5.3 Reagibilitätsmuster während der Stressbelastungsuntersuchung ..............................68

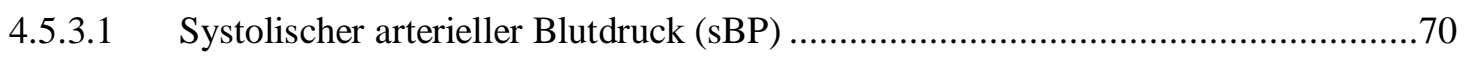

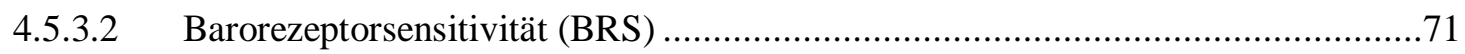

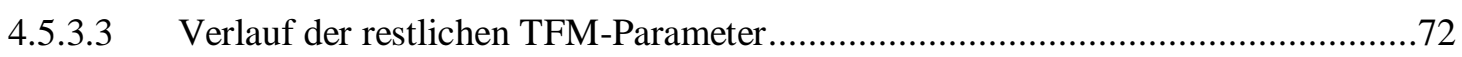

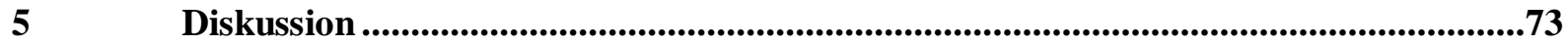

5.1 Das Patientinnenkollektiv vor dem Hintergrund der Komplexität traumaassoziierter

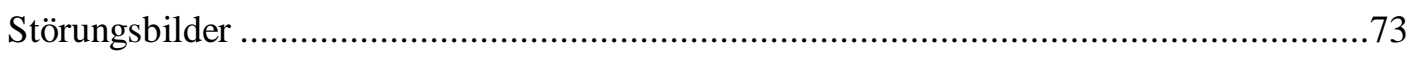

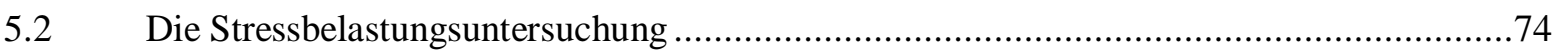

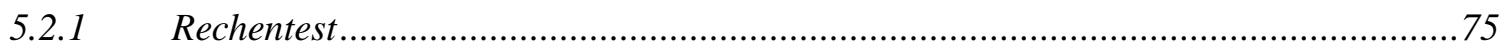

5.2.1.1 Hämodynamische Parameter während Rechnen ................................................... 76

5.2.1.2 Autonome Parameter während Rechnen ............................................................ 79

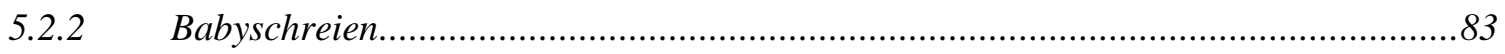

5.2.2.1 Hämodynamische Parameter während Babyschreien............................................84

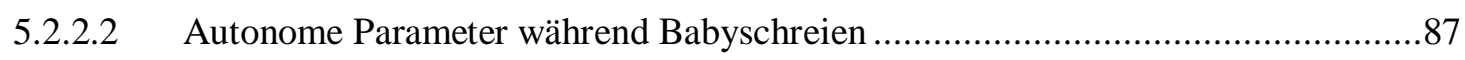

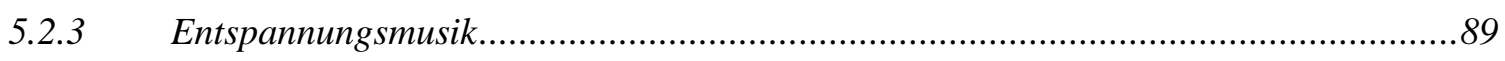

5.2.3.1 Hämodynamische Parameter während Musik ......................................................90

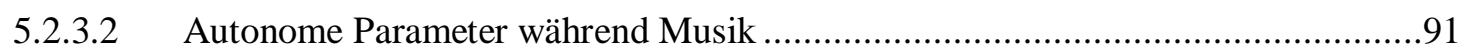

5.2.4 Rückbildungsfähigkeit nach Stressanforderung (Recovery) .....................................93

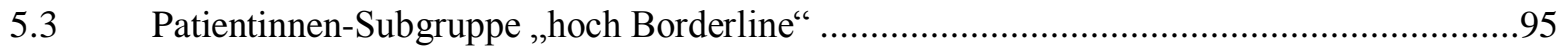

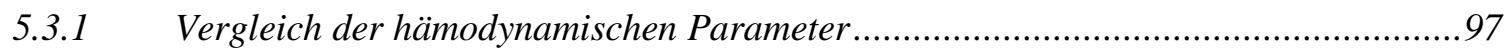

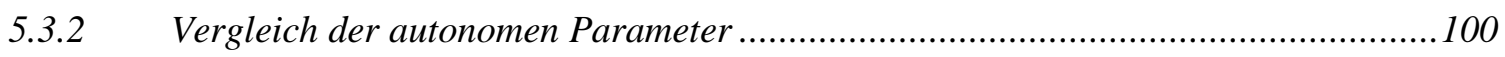

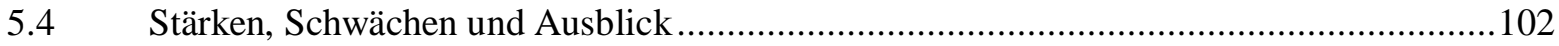

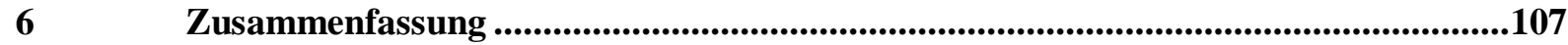




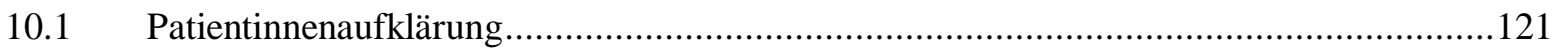

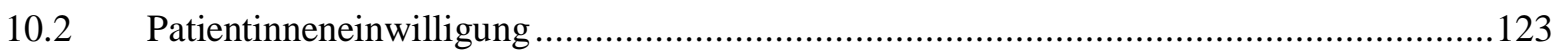

10.3 Allgemeiner Fragebogen zur Erhebung soziodemographischer Daten ...........................125 


\section{Abkürzungsverzeichnis}

AAR Adaptive Autoregressive

ACTH Adrenokortikotrophes Hormon

AFG Asklepios Fachklinikum Göttingen

ANOVA Analysis of variance

ANS

Autonomes Nervensystem

APA

American Psychiatric Association

BDI

Beck-Depressions-Inventar

BMI

Body Mass Index

$\mathrm{BP}$

Blood Pressure

BPI

Borderline-Persönlichkeits-Inventar

BPS

Borderline-Persönlichkeitsstörung

BPV

Blood Pressure Variability

BRS

Baroreceptor Reflex Sensitivity

BSL

Borderline-Symptom-Liste

$\mathrm{CO}$

Cardiac Output

CI

Cardiac Index

CRF

Corticotropin-Releasing-Factor

DA

Dissoziative Amnesie

dBP

diastolic Blood Pressure

DBT

Dialektisch-behaviorale Therapie

DDIS

Dissociative Disorders Interview Schedule

DES

Dissociative Experiences Scale

DESNOS Disorders of Extreme Stress Not Otherwise Specified

DIB Diagnostisches Interview für Borderline-Störungen

DIPS Diagnostisches Interview bei psychischen Störungen

DIS Dissoziative Identitätsstörung

DS Dissoziative Störungen

DSM Diagnostic and Statistical Manual of Mental Disorders

EKG Elektrokardiogramm

EMDR Eye Movement Desensitization and Reprocessing

FDA Food and Drug Administration

FDS Fragebogen dissoziativer Symptome

GSI Global Severity Index

HF High Frequency $(0.15-0.4 \mathrm{~Hz})$

HF-HRV Hochfrequenzband der Herzratenvariabilität 
HR

HRV

HZV

ICD-10

IES-R

IKG

I-kPTBS

IPDE

kPTBS

LF

LF-HRV

LF/HF

$\mathrm{mBP}$

NICE

PAG

PDE

PEP

PITT

PS

PTBS

PTSD

PWV

RMSSD

RRI

RSA

sBP

SCID-D

SCL-90-R

SIDDS

SIDES

SDNN

SI

SUD

SV

SVV
Herzrate

Heart Rate Variability

Herzzeitvolumen

International Statistical Classification of Diseases and Related Health Problems, 10. Revision

Impact of Event Scale, revidierte Version

Impedanzkardiographie

Interview zur Diagnostik der komplexen posttraumatischen Belastungsstörung International Personality Disorder Examination

komplexe posttraumatische Belastungsstörung

Low Frequency $(0.04-0.15 \mathrm{~Hz})$

Niederfrequenzband der Herzratenvariabilität

Herzratenvariabilitäts-Ratio

mean Blood Pressure

National Institute of Clinical Excellence

periaquaeduktales Höhlengrau

Personality Disorder Examination

Pre Ejection Period

Psychodynamische Imaginative Traumatherapie

Persönlichkeitsstörung

Posttraumatische Belastungsstörung

Posttraumatic Stress Disorder

Pulse Wave Velocity

Root Mean Square of Successive Differences

RR-Intervall

respiratorische Sinusarrhythmie

systolic Blood Pressure

Structured Clinical Interview for DSM-IV Dissociative Disorders

Symptom-Check-Liste 90, revidierte Version

Strukturierter Interviewleitfaden zur Diagnostik dissoziativer Störungen

Structured Clinical Interview for Disorder of Extreme Stress

Standardabweichung aller RR-Intervalle

Schlagindex

Subject Units of Discomfort

Schlagvolumen

Selbstverletzendes Verhalten 
TFM

TPR

TPRI

UMG

VLF

VLM

WHO
Task Force Monitor

Total Peripheral Resistance

Total Peripheral Resistance Index

Universitätsmedizin Göttingen

Very Low Frequency $(0.0-0.04 \mathrm{~Hz})$

ventrolaterale Medulla oblongata

World Health Organization/Weltgesundheitsorganisation 


\section{$1 \quad$ Einleitung}

\section{$1.1 \quad$ Stressbewältigungssysteme}

In Stresssituationen stehen dem Körper verschiedene, im Rahmen der Evolution als überlebenssichernd bewährte Anpassungsmöglichkeiten zur Verfügung. Entgegen alten Lehrmeinungen sind heute mindestens drei verschiedene Stressbewältigungssysteme des Menschen bekannt: das Furcht-oder Kampf-Flucht-System, das Panik- oder Bindungssystem sowie das Erstarrungssystem, welche an unterschiedliche Hirnzentren gekoppelt sind, aber gleichzeitig auch eng interagieren (Panksepp et al. 1991).

\subsubsection{Furchtsystem}

Das Furchtsystem ist an die Hirnregionen Amygdala im limbischen System, Locus Coeruleus im Hirnstamm und den präfrontalen Kortex auf kortikaler Ebene gebunden und wird durch die Neurotransmitter Noradrenalin und Adrenalin erregt. In Gefahrensituationen reagiert das System mit Aktivierung des Sympathikus und bereitet uns auf Kampf oder Flucht vor. Aufmerksamkeit, Muskelspannung und Reaktionsbereitschaft steigen. Es wird daher auch als Kampf-Flucht-System bezeichnet und wurde zunächst von Cannon und später von Selye beforscht (Cannon 1914, Selye 1953, Sachsse 2003). Im Idealfall kann man auf bereits erprobte Lösungsstrategien zurückgreifen und das Furchtzentrum wird durch Dopamin und Opiate beruhigt. Das Furchtsystem leistet daher einen entscheidenden Beitrag für unsere Kognitionsfähigkeit und Lernprozesse (Hüther 1996). Findet sich jedoch keine Problemlösung und das Furchtsystem bleibt zu lange oder zu intensiv erregt, dann wird über den Hypothalamus die humorale Stressachse aktiviert. Über Corticotropin-Releasing-Factor (CRF) aus dem Hypothalamus wird eine Kaskade mit ACTH (Adrenokortikotrophes Hormon)- und Betaendorphin-Freisetzung aus der Hypophyse in Gang gesetzt, die letztendlich in einer CortisolAusschüttung der Nebennierenrinde mündet. Cortisol wirkt beruhigend auf das Furchtsystem, kann jedoch bei langanhaltender Ausschüttung durch Dauerstress auch negative Auswirkungen wie beispielsweise den Verlust bewährter Lösungsstrategien und Immunschwäche zur Folge haben (Yehuda 2002).

\subsubsection{Paniksystem}

Das Paniksystem hingegen wird aktiviert, wenn sich Säuglinge oder Kleinkinder von der Mutter alleingelassen fühlen. Solch eine Hilflosigkeit hervorrufende Situation führt bei Tieren zu klagenden Lauten und beim Menschen zu Weinen bzw. Schreien. Diese sogenannten Distress Vocalisations rufen in der Mutter eine Stresssituation hervor, die sie durch ihre Anwesenheit, Körperkontakt, „Stillen“ und Nähe beenden kann, womit sie auch die Bindung zum Kind fördert (Hüther und Sachsse 2007). Panksepp et al. (1991) vermuten in diesem System sowohl eine Verbindung zur sozialen Bindung als 
auch zur Ausprägung des Herdenverhaltens, weshalb es auch als Bindungssystem bezeichnet wird. Wesentlich beteiligte Hirnzentren sind das periaquaeduktale Höhlengrau (PAG) in der Formatio reticularis, das laterale Septum sowie der Gyrus cinguli. Aktiviert wird das Paniksystem durch den Neurotransmitter Glutamat, wodurch die Aktivität des Parasympathikus gesteigert wird. Als Erwachsene spüren wir dann die vegetativen Symptome der Angst: Kloß im Hals, Druck auf der Brust, weiche Knie, Zittern und Verfallen in eine unterwürfige Demutshaltung (Hüther und Sachsse 2007). Beruhigend wirken Oxytocin, Endorphine, Opioide aber auch Nikotin und Heroin. In neueren empirischen Studien konnte eine Korrelation des Oxytocin-Plasmaspiegels bei Müttern und deren Kindern mit dem Ausmaß von sozialem Bindungsverhalten nachgewiesen werden (Feldman et al. 2007). Eine Geborgenheit spendende Interaktion zwischen Mutter und Kind ist daher entscheidend für die Entwicklung kindlicher Stressbewältigungsstrategien.

Die beiden Alarmsysteme für Furcht und Panik sind sehr eng miteinander verknüpft und interagieren intensiv. Das Furchtsystem ist ein wichtiger Beruhigungsmechanismus für das Paniksystem, da wir mit Hilfe unserer Kognitionen mit Furcht besser umgehen können als mit Panik (Hüther und Sachsse 2007).

\subsubsection{Erstarrungssystem und Dissoziation}

Kann in lebensbedrohlich empfundenen Situationen nicht gekämpft oder geflüchtet bzw. auf Hilfe durch Bindungen zurückgegriffen werden, geraten wir in den Zustand der Ohnmacht, für den wir kein gutes angeborenes Verarbeitungssystem besitzen (Sachsse 2003). Aus dem Tierreich kennt man die Freeze-Reaktion, bei der die Tiere zwar vegetativ hochgradig erregt sind, jedoch im Totstellreflex erstarren sowie ihr Laut-/Sprachzentrum abschalten und möglichst leise atmen. Dieser sympathikotone Status des Hyperarousal wird auch mit Panikattacken des Menschen in Verbindung gebracht (Brandao et al. 2008).

Menschen in Lebensgefahr steht noch ein weiterer Mechanismus zur Verfügung: die Dissoziation (siehe Kapitel 1.4.3, Seite 14). Darunter versteht man sowohl einen Rückzug von der Außenwelt als auch eine Reduktion der Reize nach innen. Die eigene Person (Depersonalisation) und die AußenWirklichkeit (Derealisation) werden verändert wahrgenommen. Diese parasympathikotone, hypometabolische Regulationsstrategie dient in hilflosen Stresssituationen dem Abschalten und der Schmerzlinderung, indem endogene Opiate vermehrt freigesetzt werden. Distress Vocalisations, also Hilferufe, werden jedoch auch unterdrückt (Lanius et al. 2006, Hüther und Sachsse 2007).

\subsection{Das autonome Nervensystem und sympathovagale Dysbalance}

Aufgabe des autonomen Nervensystems (ANS) ist es, eine homöostatische Regulation des Organismus mit adäquater Anpassung u.a. von Herzfrequenz und Blutdruck an innere und äußere Einflüsse zu gewährleisten. Dies erfordert eine komplexe Interaktion zwischen Sympathikus und Parasympathikus, welche Ausdruck der sogenannten sympathovagalen Balance ist (Thayer und Lane 2000). Sympathische und vagale Einflüsse auf die Sinusknotenfrequenz werden durch verschiedene, über 
Rückkopplungsmechanismen kontrollierte Oszillatoren moduliert. Dem dienen Regelsysteme, wie das respiratorische und das vasomotorische Zentrum in der Medulla oblongata, mit dem Hypothalamus als übergeordnetem Zentrum. Somit beeinflussen die Oszillatoren direkt die autonome Aktivität beispielsweise durch mechanische Abläufe (Atmung) oder andere Reflexmechanismen wie den Blutdruck regulierenden Baroreflex (siehe Kapitel 1.2.1.2, Seite 5) (Task Force 1996). Sind Sympathikus und Parasympathikus in einem dynamischen Gleichgewicht mit parasympathischer Dominanz und guter Anpassungsfähigkeit an physische sowie psychische Belastungen, so gilt dies als günstig für den gesundheitlichen Zustand (Thayer und Lane 2000). Viele somatische und psychische Krankheitsbilder werden mit einer Dysregulation des ANS in Verbindung gebracht. Dabei gilt Stress als entscheidender Faktor bei der Beeinflussung der psychophysiologischen Homöostase und damit auch bei der Entstehung, Manifestation und Aufrechterhaltung von Erkrankungen (Herrmann-Lingen 2000). Auch für die posttraumatische Belastungsstörung (PTBS) gibt es Hinweise auf eine gestörte sympathovagale Balance (siehe Kapitel 1.3.4, Seite 9).

Zur Beurteilung der Modulation der autonomen Aktivität werden hämodynamische und autonome Parameter sowohl in Ruhe als auch deren Reaktivität unter Stressbedingungen sowie die Rückbildungsfähigkeit nach Anforderung betrachtet. Als besonders ungünstig gilt eine sympathovagale Dysbalance mit Überwiegen der sympathischen Aktivität und gleichzeitig erniedrigtem Vagotonus (Thayer und Lane 2000):

- dauerhafte sympathische Hyperaktivität in Ruhesituationen (Baseline)

- hyperreaktive Sympathikusaktivierung bei Stressanforderung (Reactivity)

- nach Stressoreinwirkung keine adäquate Herabregulierung des Sympathikus bzw. eine verzögerte Abnahme des Erregungsniveaus (Recovery).

\subsubsection{Indizes sympathischer und vagaler Aktivität}

Es gibt eine Vielzahl von nicht-invasiven Markern, um den vegetativen Tonus und eine kardiovaskuläre Aktivierung zu überprüfen und darzustellen. Herrmann-Lingen et al. (2010) weisen in einer aktuellen Studie auf die Vielseitigkeit der stressinduzierten, autonomen Aktivierung hin und warnen davor, den allgemein üblichen Begriff „sympathische Aktivierung“ undifferenziert zu verwenden. Vielmehr sollte der Effekt von Stress auf die kardiovaskuläre Regulation auf vier verschiedenen Ebenen betrachtet werden. Dabei sollte zunächst zwischen alpha- und beta-adrenerger Sympathikus-Aktivierung unterschieden werden und sowohl frequenzabhängige als auch nichtrhythmische, zeitabhängige Analysen der Herzratenvariabilität in die Betrachtung eingeschlossen werden. Vorwiegend alpha-adrenerge Marker sind unter anderem systolischer Blutdruck (sBP) und peripherer Gefäßwiderstand (TPR), eine vorwiegend beta-adrenerge Aktivierung wird beispielsweise durch die Herzfrequenz (HR), das Herzzeitvolumen(HZV) oder die Preejektionsperiode (PEP) widergespiegelt. Die Variabilität der Herzrate (HRV) sowie des systolischen Blutdrucks (BPV) können sowohl im Zeit- als auch im Frequenzbereich analysiert werden. Als Indizes für die autonome 
Aktivität haben sich vor allem das Hoch- und das Niederfrequenzband der Herzratenvariabilität (HFbzw. LF-HRV) und im Zeitbereich die Standardabweichung aller RR-Intervalle (SDNN) bewährt. Ein weiterer Marker für die sympathovagale Balance ist die Barorezeptorsensitivität (BRS).

Im Rahmen von Stressreagibilitätsuntersuchungen hat sich der Task Force Monitor, ein gut evaluiertes, nicht-invasives Monitoringsystem zur Erfassung hämodynamischer und autonomer Parameter (Fortin et al. 2001), bewährt (Lache 2006, Herrmann-Lingen et al. 2010). Allgemein übliche und in zahlreichen psychophysiologischen Studien eingesetzte Stressoren sind Rechentests (Mental Arithmetic Stress Test) und Ärgerexposition (Anger Recall), wobei das Kopfrechnen einen kognitiven Stressstimulus darstellt und durch die Ärgerexposition eine emotionale Stressbelastung ausgelöst werden soll (Lache 2006). Im Rahmen von Studien zur Stressphysiologie an PTBSKollektiven sind trauma-relevante Stimuli (Trauma Recall) weit verbreitet (Cohen et al. 2000, Keary et al. 2009).

Während eines Rechentests zeigten nicht-psychiatrische Patientengruppen eine Aktivierung des sympathischen Systems in Form einer Zunahme von Herzfrequenz, Blutdruck und Gefäßwiderstand sowie eine Herabregulation der vagalen Aktivität in Form einer Abnahme der HF-HRV sowie der SDNN (Lache 2006). In der vorliegenden Arbeit wird abgeleitet aus diesen Ergebnissen ein vergleichbares Reaktivitätsmuster auch bei einem psychotherapeutischen Patientinnenkollektiv im Rahmen eines Rechentests erwartet.

Im Vergleich zum Reaktionsmuster während einer kognitiven Stressbelastung (Rechnen) löste eine Ärgerexposition als emotionaler Stimulus bei nicht-psychiatrischen Patienten eine stärkere Zunahme der vorwiegend alpha-adrenerg modulierten Parameter Blutdruck und Gefäßwiderstand und einen deutlicheren vagalen Rückzug mit Abnahme von HF-HRV und SDNN aus. Die eher beta-adrenerg modulierten Indizes Herzfrequenz und Herzzeitvolumen wurden hingegen weniger stark gesteigert (Lache 2006).

In der vorliegenden Studie wurde als emotionaler Stressor „Babyschreien“ gewählt. Bei gesunden Frauen stellt Babyschreien im Sinne von Distress Vocalisations einen potenten Stimulus dar, um eine emotionale physiologische Stressreaktion auszulösen (Del Vecchio et al. 2009). Es wurde daher vermutet, dass Babyschreien insbesondere bei den hier untersuchten, vulnerablen PTBS-Patientinnen eine emotionale Stressbelastung hervorrufen kann. Es gilt zu überprüfen, ob durch Babyschreien ein vergleichbares Reaktivitätsmuster wie während einer Ärgerexposition aktiviert wird.

\subsubsection{Herzratenvariabilität (HRV)}

Eine gesunde autonome Funktion spiegelt sich in einem Gleichgewicht zwischen Sympathikus und Parasympathikus wider. Dabei zeigen die RR-Intervalle bei normalem Sinusrhythmus unter Einfluss des ANS spontane Fluktuationen im EKG. Diese Variation der Herzfrequenz bezeichnet man als Herzratenvariabilität (HRV) und diese lässt sich als eine permanente Anpassungsreaktion der Herzfrequenz an externe und interne Reize und somit an die Bedürfnisse des Gesamtorganismus verstehen. Die HRV ist demnach eine Messgröße der neurovegetativen Aktivität und der autonomen 
Funktionalität und Adaptationsfähigkeit des Herzens (Malik und Camm 1993). Zur Bestimmung der HRV werden im EKG die Variationen der Abstände zweier aufeinander folgender QRS-Komplexe (RR-Intervall) beurteilt. Im Rahmen einer Zeitreihenanalyse der RR-Intervalle lässt sich die HRV sowohl im Zeit- (Time-Domain) als auch im Frequenzbereich (Frequency-Domain) berechnen (siehe Kapitel 3.7.3, Seite 30). Eine gesunde Herzschlagfolge ist durch eine ausgeprägte Variabilität der Herzfrequenz geprägt, wohingegen eine sehr eingeschränkte HRV als pathologisch im Sinne einer Dysfunktion des ANS anzusehen ist. In Ruhe dominiert der vagale Einfluss auf das Herz, während Stresserleben zu einem Absinken der HRV durch Steigerung der sympathischen bzw. Abfall der parasympathischen Aktivität führt (Task Force 1996). Inzwischen sind viele Faktoren bekannt, die in der Lage sind, die HRV bzw. die sympathovagale Balance zu beeinflussen. Nach Löllgen (1999) müssen u.a. Körperlage, Alter, Geschlecht, Atmung, Trainingszustand, Belastungen, Valsalva- und ähnliche Manöver, Tageszeit und Medikamente als Einflussfaktoren beachtet werden. Chronischer Stress führt zu einer geringeren Anpassungsfähigkeit an Belastungen und kann sich in einer reduzierten HRV äußern. Darüber hinaus haben psychologische Faktoren und persönlichkeitseigene Verarbeitungsmechanismen langfristig einen Einfluss auf die HRV (Herrmann-Lingen 2000). Bei vielen kardiovaskulären Erkrankungen, v.a. Myokardinfarkt, koronarer Herzerkrankung, Hypertonus oder Arrhythmien aber auch bei psychiatrischen Krankheitsbildern wie Depression (Ehrenthal et al. 2010a) oder Panikstörungen (Blechert et al. 2007) ist die HRV verringert und reflektiert eine Verschiebung des sympathovagalen Gleichgewichtes in Richtung Sympathikus mit einem entsprechenden Abfall des Vagotonus. Dass solch eine chronische Imbalance des ANS mit einer erhöhten Morbidität sowie einem gesteigerten Mortalitätsrisiko einhergeht, konnten zahlreiche Studien belegen (La Rovere et al. 1998, Tsuji et al. 1996, Ladwig et al. 2008).

\subsubsection{Baroreflexsensitivität (BRS)}

Zur Aufrechterhaltung der Organperfusion bei kurzfristigen Blutdruckschwankungen dient der über Pressorezeptoren vermittelte Kreislaufreflex, auch Barorezeptorreflex genannt. Die in der Wand des Karotissinus und des Aortenbogens befindlichen Barorezeptoren registrieren sowohl die Höhe des arteriellen Mitteldrucks als auch Änderungen des Blutdrucks und werden in Abhängigkeit von der Gefäßdehnung erregt. Die Afferenzen der Barorezeptoren üben einen ständigen inhibitorischen Einfluss auf sympathische Kerngebiete in der ventrolateralen Medulla oblongata (VLM) aus. Zusätzlich werden vagale Efferenzen, die dem Nucleus ambiguus in der Medulla oblongata entspringen, stimuliert. Ein Blutdruckanstieg bewirkt über den Baroreflex eine Hemmung des Sympathikus sowie eine Aktivierung des Parasympathikus mit dem Effekt der Abnahme des arteriellen peripheren Widerstandes und der Herzfrequenz. Durch eine unterschiedliche Intensität in der Aktivierung der Barorezeptoren wird der reziproke Effekt auf Sympathiko- und Vagotonus moduliert (Klinke et al. 2005). Die Baroreflexsensitivität (BRS) stellt die Empfindlichkeit des oben beschriebenen Kreislaufreflexes dar und gilt als Index des kardialen sympathovagalen Gleichgewichtes. Die BRS spiegelt die Beziehung zwischen Veränderungen von Bludruck und RR- 
Intervall wider und gibt Auskunft über den autonomen Funktionsstand. Eine erniedrigte BRS ist mit einer eingeschränkten vagalen Modulation assoziiert und wird daher als prognostisch ungünstiger Faktor diskutiert (La Rovere et al. 1995, 1998). Als wichtigster, potentiell steigernder Einflussfaktor auf die BRS ist die Atmung zu nennen (Bernardi et al. 2002). Die BRS lässt sich u.a. mittels Sequenzmethode (Parati et al. 1988) bestimmen (siehe Kapitel 3.7.4, Seite 31).

\subsection{Posttraumatische Belastungsstörung (PTBS)}

Bei der Entstehung psychoreaktiver Störungen geht man davon aus, dass es infolge einer Überflutung von plötzlichen, bedrohlichen und aversiven Reizen zur Überforderung der individuellen Bewältigungsmöglichkeiten kommt. Daher lässt sich die posttraumatische Belastungsstörung (PTBS) heute als psychosomatische, vielleicht sogar als somatopsychosomatische Erkrankung des Stressverarbeitungssystems bezeichnen und diese hat in den letzten Jahren verstärkt das klinische und wissenschaftliche Interesse geweckt. Die angloamerikanische Wortwahl Posttraumatic Stress Disorder (PTSD) charakterisiert dieses Störungsbild als Stress-Verarbeitungsstörung präziser als die deutsche Bezeichnung.

\subsubsection{Klinische Merkmale der PTBS - Maladaptive Stressbewältigung}

Ein klar definiertes Krankheitskonzept unter dem Begriff PTBS wurde erstmals 1980 in das Diagnostic and Statistical Manual of Mental Disorders DSM-III (APA 1980) aufgenommen und beschreibt eine gravierende psychische Störung, die nach außergewöhnlichen belastenden Erfahrungen auftreten kann. In den aktuellen Diagnosesystemen des DSM-IV (APA 2003) und der ICD-10 (WHO 2006) wird für die Diagnose PTBS als ätiologisch gesicherter Faktor das Erleben eines schwer belastenden Ereignisses vorausgesetzt (Kriterium A). Die betroffene Person muss eine Situation erlebt und/oder beobachtet haben, die Tod, Lebensgefahr, schwere Körperverletzung oder Gewalt beinhaltete oder bei der die körperliche Unversehrtheit der eigenen oder einer anderen Person bedroht gewesen ist. Jedoch führt nicht jede Extrembelastung bei allen Betroffenen zu einer manifesten oder länger anhaltenden psychischen Störung. Das traumatisierende Potential hängt von bestimmten Merkmalen des Stressors und dessen Kontext ab (NICE 2005). Dazu zählen vor allem die Dauer der Einwirkung (punktuell oder wiederholt), der Betroffenheitsgrad (direkt oder indirekt) und die Art des Ursprungs (menschlich bzw. sozial oder nicht-menschlich). Die Wahrscheinlichkeit, eine PTBS zu entwickeln, ist nach Traumata, die intentional durch Mitmenschen verursacht werden, deutlich höher als beispielsweise nach Naturkatastrophen. Des Weiteren spielen der unerwartete oder unvermeidbare Charakter des Traumas sowie die fehlende Kontrolle über zukünftige Wiederkehr bzw. mangelnde Möglichkeiten der Verhinderung eine entscheidende Rolle. Oftmals ist dies verbunden mit Gefühlen massiver Angst und Hilflosigkeit. Fischer und Riedesser definieren das psychische und psychosomatische Trauma als „ein vitales Diskrepanzerlebnis zwischen bedrohlichen Situationsfaktoren und individuellen Bewältigungsmöglichkeiten, das mit Gefühlen von Hilflosigkeit und schutzloser Preisgabe einhergeht und so eine dauerhafte Erschütterung von Selbst- und 
Weltverständnis bewirkt“ (Fischer und Riedesser 1998, Seite 79). Diese Aussage unterstreicht die Bedeutung des subjektiven Erlebens während traumatischer Situationen. In der Regel können Menschen nach traumatisierenden Ereignissen auf ein angeborenes soziales Selbstheilungsprogramm bestehend aus Bindungserfahrungen und sozialer Sicherheit mit Unterstützung und Geborgenheit zurückgreifen. Sind diese Verarbeitungsmechanismen jedoch gestört oder nicht vorhanden, so kann sich abhängig vom traumatisierenden Potential des Stressors eine chronische posttraumatische Stressverarbeitungsstörung entwickeln (NICE 2005). Zwar sind die Reaktionen auf ein Trauma vielfältig, können jedoch übergeordneten Symptommustern zugeordnet werden, deren Vorliegen mindestens einen Monat nach dem traumatisierenden Ereignis fortbestehend für die Diagnose PTBS unabdingbar ist (APA 2003, WHO 2006). Die Kernsymptome der PTBS stellen eine Symptomtrias dar (Abbildung 1, Seite 7).
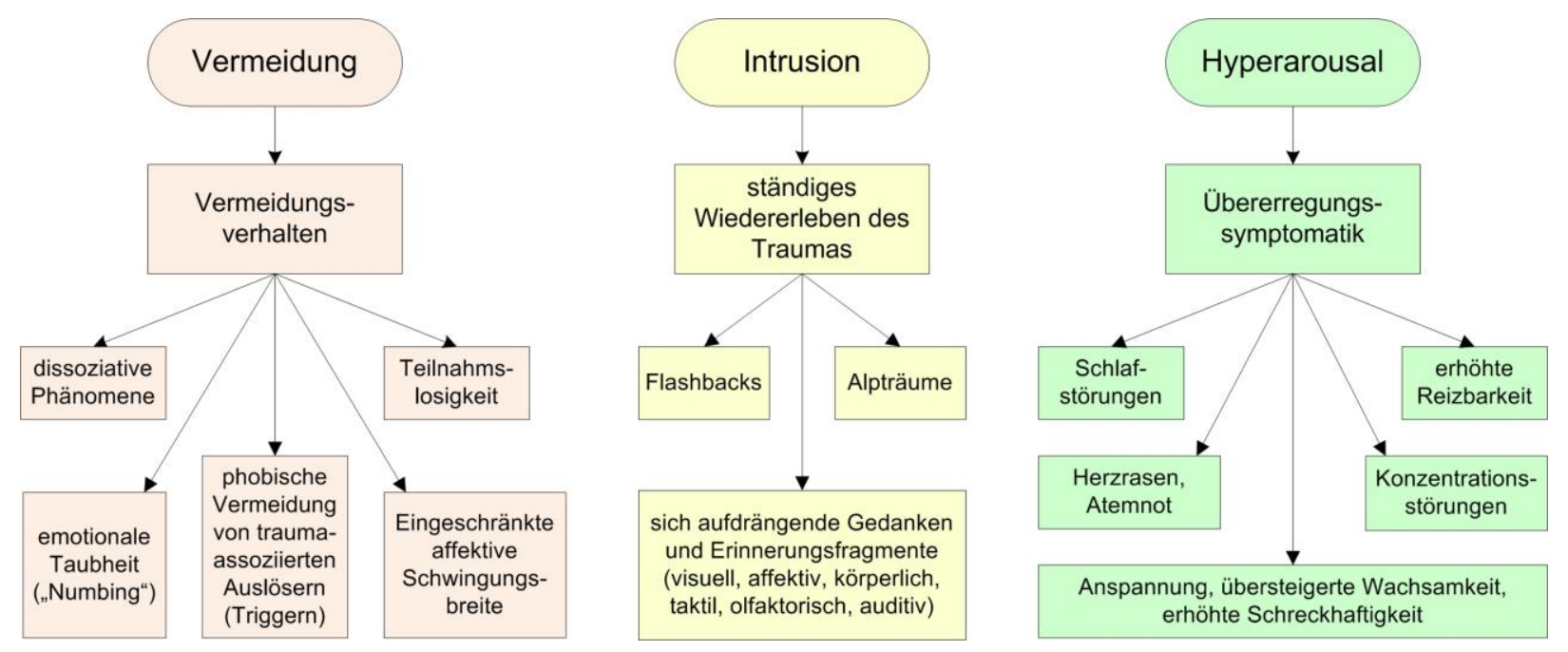

Abbildung 1: Hauptsymptomatik der posttraumatischen Belastungsstörung

Der Alltag der PTBS-Patienten ist geprägt von immer wiederkehrenden Flashbacks (Bilder) und Intrusionen (Gedanken und Affekte). Dies beruht auf einer nur fragmenthaften Speicherung der traumatisierenden Erfahrungen, ohne den typischen Prozess der Langzeitgedächtnisbildung zu durchlaufen. Zudem wird das Priming-Gedächtnis aktiviert, welches grobrastig auf Ähnlichkeiten und Ergänzungen anspricht, wodurch eine Sensibilisierung auf Trigger-Reize erfolgt (Sachsse et al. 2009). Die Problematik besteht darin, dass solche Trigger-Reize generalisieren können und somit ubiquitär werden. Die Patienten zeigen ein typisches Erscheinungsbild mit erhöhter Reizbarkeit und Schreckhaftigkeit, ein sogenanntes Hyperarousal, und sind von einem Vermeidungsverhalten dominiert, um nicht ständig durch Trigger-Reize in Hyperarousal-Situationen zu kommen.

Als Folge langjähriger unverarbeiteter Traumatisierungen in der Kindheit und Jugend kann sich die posttraumatische Störung als Persönlichkeitsstörung, insbesondere als BorderlinePersönlichkeitsstörung (BPS) manifestieren (Sachsse et al. 1997, Sachsse 2002). Nicht jede BPS ist eine Trauma-Folgestörung, aber Traumatisierungen sind zumindest bei 50 bis $70 \%$ der BPS-Patienten 
ein wesentlicher ätiologischer Faktor (Zanarini et al. 1997). Eine langfristig bestehende Symptomatik birgt die Gefahr, komorbide psychische Störungen zu entwickeln, wie beispielsweise Depressionen, Angst- oder Panikstörungen, somatoforme Störungen, dissoziative Störungen oder Suchterkrankungen. Neben dem bei der PTBS typischen Abwehrstil, der durch Hyperarousal gekennzeichnet ist, beschreibt Lanius einen dissoziativen Abwehrstil, welcher demgegenüber durch Depersonalisation, Derealisation und ein „Ausklinken“ charakterisiert ist (Lanius et al. 2006). Im Sinne einer Freeze-Reaktion berichten PTBS-Patienten oft über emotionale Erstarrung (Numbing). Weitere häufige Begleiterscheinungen der PTBS sind Konzentrationsschwierigkeiten, emotionale Labilität, Kopfschmerzen und Schwindelgefühle. Mit der Vermeidungstendenz gehen oftmals Impulshandlungen wie z.B. plötzliche Ortswechsel einher. Substanzmissbrauch (Alkohol, beruhigende Medikamente) lässt sich als Versuch verstehen, die unerträglichen Erinnerungen zu unterdrücken und die innere Angespanntheit zu reduzieren, Suizidhandlungen als Folge überstarker Schuldgefühle. Interessenverlust, sozialer Rückzug, Erschütterung des Bedeutungs- und Wertesystems bezüglich (Selbst-)Sicherheit und (Selbst-)Vertrauen, Scham- und/oder Schuldgefühle sind häufige Merkmale einer PTBS. Diese funktionellen und sozialen Einschränkungen betreffen auch in erheblichem Maße die Ebenen Beruf, Familie und Teilhabe am sozialen Leben (Sachsse 2002).

In der Folge von psychischen Traumatisierungen werden hormonelle, neuroanatomische sowie psychophysiologische Veränderungen beforscht, welche in den folgenden Abschnitten kurz vorgestellt werden sollen. Schwere Traumata scheinen somatische Veränderungen zu hinterlassen. Dieser Problematik ist auch die vorliegende Dissertation gewidmet.

\subsubsection{Endokrinologische Dysfunktion bei PTBS}

Zum klinischen Bild des Hyperarousal wären permanent erhöhte Stresshormonspiegel zu erwarten gewesen, jedoch stellte die Arbeitsgruppe um Yehuda bei PTBS-Patienten mit lange zurück liegenden traumatisierenden Ereignissen signifikant erniedrigte Cortisolwerte im Plasma bzw. 24-StundenSammelurin im Vergleich zu Gesunden und einer psychiatrischen Vergleichspopulation fest (Yehuda et al. 1995, Stein et al. 1997, Yehuda 2002). Ein Dexamethasonhemmtest mit der Hälfte der Standarddosis ergab signifikant niedrigere Cortisol-Plasmakonzentrationen bei den PTBS-Patienten. Während akute Stressreaktionen zu einer verstärkten Glukokortikoidfreisetzung führen, scheinen chronische Stressreaktionen eine dauerhafte Senkung des basalen Cortisolspiegels und eine rasche Absenkung nach erneuter Stressbelastung hervorzurufen. Möglicherweise liegt hier wie bei anderen chronischen Stresssyndromen im Verlauf eine Erschöpfung der kortikotropen Achse und damit das Fehlen eines wichtigen Feedbackmechanismus zur Dämpfung der Stressreaktion bei PTBS vor.

Des Weiteren wird diskutiert, inwiefern der verfügbare Cortisolspiegel während einer Traumatisierung die Entstehung einer akuten sowie auch einer chronischen PTBS beeinflusst und es wird nach einer Verbindung zwischen (neuro-)endokrinen Merkmalen und bestimmten Copingmustern gesucht (Yehuda 2002). 


\subsubsection{Neurobiologische Modelle zur PTBS}

Erste Studien der Arbeitsgruppe Bremner et al. $(1995,1997)$ an Vietnam-Veteranen und an in der Kindheit missbrauchten Frauen mit chronifizierter PTBS-Symptomatik konnten eine deutliche, mittels Magnetresonanztomographie festgestellte Verkleinerung des Hippokampus bei den Betroffenen nachweisen. Diese bahnbrechenden Ergebnisse konnten durch zahlreiche Studien bestätigt werden (Karl et al. 2006a) und warfen die zentrale Frage auf, ob Traumatisierungen obligatorisch zu messbaren strukturellen Gehirnveränderungen führen. Im Rahmen weiterer Untersuchungen konnte gezeigt werden, dass eine Atrophie des Hippokampus keineswegs Trauma- bzw. PTBS-spezifisch ist, sondern auch Personen mit anderen psychischen Störungen wie beispielsweise Depressionen, Zwangsstörungen, Schizophrenie, Alkoholismus oder verschiedenen neurologischen Erkrankungen sowie einer Cortison-Therapie betroffen sind (Geuze et al. 2005). Schädigungen der Hippokampusformation sind im Allgemeinen mit kognitiven Defiziten vergesellschaftet. Auch bei der PTBS leiden die Patientinnen oft unter erheblichen Gedächtnisstörungen. Das Ausmaß der Hippokampusatrophie geht dabei mit dem Schweregrad der Gedächtnisdefizite einher (Bremner et al. 1995, 1997; Irle et al. 2009; Weniger et al. 2008). Signifikante hippokampale Volumenreduktionen treten erst nach einer gewissen Latenzzeit von mehr als 10 Jahren PTBS-Symptomatik auf, korrelieren dann jedoch mit dem Ausmaß der Belastung (Irle et al. 2009). Es könnte daher vermutet werden, dass eine ausgeprägte Hippokampusatrophie durch eine lange Zeit persistierende und schwere PTBSSymptomatik begünstigt wird.

Neben dem Hippokampus stehen auch andere Hirnregionen bei der PTBS im Fokus der Aufmerksamkeit, wie die Amygdala und der Gyrus cinguli, die mit unseren Stressverarbeitungssystemen in Verbindung gebracht werden (siehe Kapitel 1.1, Seite 1). Eine Verkleinerung von Amygdala sowie cingulärem Kortex konnte im Rahmen einer PTBS bzw. komorbider BPS festgestellt werden (Schmahl et al. 2003, Irle et al. 2009). Des Weiteren konnten bei Personen mit PTBS bzw. BPS mittels funktioneller Magnetresonanz-Untersuchungen Veränderungen neuronaler Aktivitätsmuster nachgewiesen werden. Während die für die Furchtreaktion verantwortliche Amygdala im Rahmen von Provokationsstudien erhöhte Aktivitätsmuster zeigt, sind frontale und cinguläre Kortexareale sowie die Broca-Region (Sprachzentrum) vermindert aktiviert (Lanius et al. 2006). Diese Minderaktivierung des Sprachzentrums könnte als Korrelat einer traumareaktiven Sprachlosigkeit sowie der Unfähigkeit, die traumatischen Erlebnisse in ein verbal vermitteltes Langzeitgedächtnis zu überführen, interpretiert werden.

\subsubsection{Gestörte physiologische Stressreagibilität bei PTBS}

Bereits seit 1871 ist durch die Forschungsergebnisse von Da Costa an Soldaten bekannt, dass traumatisierte Patienten ein ausgeprägtes Muster an erhöhten psychophysiologischen Reaktionen zeigen (Da Costa 1871). Mittlerweile gibt es viele empirische Daten, die erhöhte Basisparameter sowie eine gesteigerte Reaktivität bei PTBS-Patienten bestätigen. In einer aktuellen Meta-Analyse von 
Pole (2007) wurden einige Studien zur psychophysiologischen Reaktivität bei Patienten mit PTBS sowohl in Ruhesituationen als auch unter Traumaexposition verglichen. Zusammenfassend stellte sich bei den PTBS-Patienten im Vergleich zu Gesunden ein erhöhtes psychophysiologisches Erregungsniveau - insbesondere eine erhöhte Herzfrequenz - heraus. Orr und Roth (2000) fanden beispielsweise bei PTBS-Patienten eine erhöhte Ruhe-HR sowie eine gesteigerte Reaktivität der HR mit überschießender Sympathikusantwort bei Traumakonfrontation. Ähnliche Ergebnisse konnten auch für Vietnam-Veteranen (Keane et al. 1998) und Motorradunfallopfer (Blanchard et al. 1996) mit PTBS gezeigt werden. In beiden Studien wurde darüber hinaus über die Vorhersagbarkeit einer PTBS anhand der HR-Reaktion bei Traumaexposition diskutiert. Andere oft replizierte Ergebnisse offenbarten eine erhöhte Schreckreaktion auf laute Töne oder andere unangenehme Stimuli (Metzger et al. 1999) sowie eine verminderte Habituationsfähigkeit bei wiederholter Stimuli-Exposition (Shalev et al. 1992).

Hyperarousal können als fehlende inhibitorische Kontrolle mit niedriger Parasympathikus-Aktivität gegenüber einem erhöhten Sympathikotonus verstanden werden (Sack et al. 2004, Hopper et al. 2006). Ein hoher Parasympathikotonus kann daher ein Indikator für einen intakten inhibitorischen Feedbackmechanismus sein. Er ist assoziiert mit einer besseren Kapazität zur Selbstregulation und Stressbewältigung. Im Umkehrschluss hat ein erniedrigter Vagotonus eine gestörte Stressbewältigung zur Folge. Im letzten Jahrzehnt rückte daher zunehmend die Spektralanalyse der Herzfrequenzvariabilität (HRV) bei Angststörungen und insbesondere bei der PTBS in das Interesse der Forschung. Verschiedene Studien haben einen erniedrigten Vagotonus bei Patienten mit PTBS nachgewiesen (Cohen et al. 1997, 1998, Sack et al. 2004, Keary et al. 2009, Hopper et al. 2006) und dabei erwies sich die Spektralanalyse der HRV als geeignete, nicht-invasive Methode um kardiovaskuläre autonome Regulationsmechanismen beurteilen sowie die dynamische Interaktion von Sympathikus und Parasympathikus abbilden zu können. Cohen et al. $(1997,1998)$ fanden als Zeichen eines autonomen Hyperarousal bei einer kleinen Stichprobe von neun PTBS-Patienten eine erhöhte Herzfrequenz sowie eine erniedrigte HRV mit Sympathikusdominanz (hohe LF-HRV) und abgeschwächtem Vagotonus (niedrige HF-HRV) in Ruhe. Darüber hinaus zeigten die Patienten bei der Aufforderung, über ihr traumatisches Erlebnis zu berichten, keine weitere autonome kardiovaskuläre Reaktion (Cohen et al. 1998). Die autonome Überaktivität der Patienten in Ruhe war dabei vergleichbar mit der Reaktion der gesunden Kontrollgruppe unter Stressanforderung. Cohen et al. (1998) interpretierten die Ergebnisse als Unfähigkeit, eine adäquate kardiovaskuläre Anpassung an Stresssituationen hervorrufen zu können, da bereits in Ruhe eine enorm erhöhte autonome Aktivierung mit fehlendem vagalen Einfluss vorliegt. Eine Studie von Sack et al. aus dem Jahre 2004 untersuchte den Einfluss einer niedrigen respiratorischen Sinusarrythmie (RSA) als Indikator des kardialen Vagotonus sowie die Reaktion der HR bei Traumakonfrontation. Alle Studienteilnehmer mit PTBSDiagnose zeigten einen signifikanten HR-Anstieg und gleichzeitig einen Abfall der RSA bei traumaassoziiertem Stimulus. PTBS-Patienten mit niedriger RSA in Ruhe wiesen zudem einen 
stärkeren und zeitlich verlängerten HR-Anstieg bei Traumakonfrontation und einen langsameren Rückgang der Werte in der Erholungsphase auf (Sack et al. 2004). Auch Keary et al. (2009) registrierten bei Vorliegen einer PTBS eine reduzierte parasympathische Regulationsfähigkeit im Sinne einer erniedrigten HF-HRV während Stressanforderungen (Trauma Recall und Rechentest). Der erniedrigte kardiale Vagotonus scheint demnach für die bei der PTBS typischen pathophysiologischen Arousals sowie gestörte Affektregulation verantwortlich zu sein.

Zusammenfassend muss festgehalten werden, dass alle bisherigen Studien zur Regulationslage bei PTBS nur eine generelle sympathische Aktivierung, hauptsächlich bezüglich der Herzfrequenz, beschrieben und nicht zwischen alpha- und beta-adrenerger Aktivierung unterschieden. Zudem sind die Studienergebnisse zur sympathovagalen Dysregulation bei PTBS nicht einheitlich. Der Stellenwert der HRV zur Beurteilung der sympathovagalen Balance wurde nach anfänglicher Euphorie etwas relativiert. Dass dieses Verfahren methodische Grenzen und Fehlerquellen aufweist, sollte bei einer Interpretation stets bedacht werden.

\subsection{Weitere traumaassoziierte Störungsbilder}

\subsubsection{Komplexe PTBS (kPTBS)}

Andauernde Persönlichkeitsveränderungen nach Extrembelastungen werden auch als komplexe posttraumatische Belastungsstörung (kPTBS) konzeptualisiert. Herman entwickelte 1992 erstmals das Konzept der kPTBS. Die Symptommuster, welche in Tabelle 1 (Seite 11) zusammenfassend dargestellt sind, zeigen hierbei eine hohe phänotypische Variabilität. Besonders gefährdet sind Opfer lang anhaltender, intentionaler Traumatisierung in der Kindheit durch nahe stehende Bindungspersonen (Heffernan und Cloitre 2000).

Tabelle 1: Kriterien der kPTBS nach Herman 1992

\section{Symptombild bei der komplexen posttraumatischen Belastungsstörung}

- $\quad$ veränderte Affektregulation (SVV, Suizidgedanken, Wut, Dysphorie)

- Bewusstseinsveränderungen (dissoziative Phänomene, Intrusionen)

- veränderte Selbstwahrnehmung (Scham-/Schuldgefühle)

- $\quad$ veränderte Wahrnehmung des Täters

- Veränderungen in der Sexualität und Beziehungsgestaltung (sozialer Rückzug, Misstrauen, unzureichender Selbstschutz)

- veränderte persönliche Werte und Glaubensvorstellungen (Hoffnungslosigkeit)

- $\quad$ Somatisierung

Charakteristisch sind eine gestörte Affekt- und Impulsregulation sowie dissoziative Phänomene, die zu erheblichen Problemen im Lebensalltag der Betroffenen führen (Sack 2004). Neben Substanzmissbrauch ist auch selbstverletzendes Verhalten (SVV) bei der kPTBS weit verbreitet. Es dient nicht nur dem Stressabbau und der Selbsttröstung, sondern vor allem auch der Beendigung 
dissoziativer Zustände und emotionaler Taubheit (Sachsse 2002). Somatische Beschwerden, Vereinsamung, Scham- und Schuldgefühle, Misstrauen gegenüber Mitmenschen, Beschäftigung mit dem Trauma als Lebensinhalt und allgemeiner Sinnverlust prägen das Bild. Hinzu kommt, dass solche Personen besonders anfällig für Retraumatisierungen sind, da sie nicht in der Lage sind, sich ausreichend selbst zu schützen (Herman 2003). Das breite Spektrum kognitiver, affektiver und psychosozialer Beeinträchtigungen wurde begrifflich gefasst als Disorder of Extreme Stress Not Otherwise Specified (DESNOS) in das DSM-IV als Forschungsdiagnose aufgenommen (APA 2003).

Miller und Resick (2007) unterscheiden zwei Verhaltensmuster bei der kPTBS: „Externalisierer“ und „Internalisierer“.

- Bei der externalisierenden Symptomatik dominiert eine gestörte Impulskontrolle, Substanzmissbrauch sowie Züge einer Cluster-B-Persönlichkeitsstörung. Dieses Bild passt zum expressiven Verhalten bei der Borderline-Persönlichkeitsstörung und wäre auf stressphysiologischer Ebene dem Kampf-Flucht-System zuzuordnen.

- Internalisierer hingegen kennzeichnet stark depressives und vermeidendes Verhalten mit Tendenz zur Selbstverletzung und Dissoziation. Dies scheint dem emotionalen Numbing im Sinne einer Erstarrungsreaktion ohne BPS-Symptomatik nahe zu kommen und wäre Hermans Konzept der kPTBS entsprechend.

Es besteht eine deutliche Überschneidung der Symptomatik zwischen der kPTBS und der BorderlinePersönlichkeitsstörung sowie dissoziativen Störungen. Auch eine gemeinsame Traumaätiologie ist zu vermuten (Zanarini et al. 1997, Overkamp und Helmke-Jaeger 2010, Sack et al. 2012). Gemeinsamkeiten und Abgrenzungsmöglichkeiten zwischen kPTBS und den beiden Krankheitsbildern werden im Folgenden näher beleuchtet.

\subsubsection{Borderline-Persönlichkeitsstörung (BPS)}

Die Abgrenzung einer (k)PTBS von einer Borderline-Persönlichkeitsstörung (BPS) ist häufig schwer, da sich die Symptome erheblich überschneiden und sehr viele Borderline-Patienten von frühen Traumatisierungen berichten. So wurden in zahlreichen Studien bei Patienten mit BPS eine extrem hohe Prävalenz an traumatischen Erfahrungen in der Kindheit in Form von sexuellem oder körperlichem Missbrauch und/oder Vernachlässigung nachgewiesen (Sachsse et al. 1997, Zanarini et al. 1997, Zanarini et al. 2006, Sack et al. 2012).

Kernberg beschrieb die BPS als eigenständige Persönlichkeitsorganisation (Kernberg 1967, Kernberg 2000). Das Symptombild einer BPS ist bunt und keinesfalls einheitlich. Kennzeichnend ist eine Instabilität der zwischenmenschlichen Beziehungen, des Selbstbildes und der Affektregulation. Laut DSM-IV müssen für die Diagnose BPS mindestens 5 von 9 Kriterien aus Tabelle 2 (Seite 13) erfüllt sein (APA 2003). Eine Komorbidität bzw. Überlappung von BPS und traumaassoziierten Störungen und die daraus resultierenden negativen Auswirkungen auf Verlauf und Prognose sind bekannt (Zanarini et al. 2006). Spitzer et al. (2000) untersuchten 30 stationär behandelte Patienten mit BPS 
hinsichtlich traumatischer Kindheitserfahrungen, komorbider PTBS, dissoziativer Psychopathologie und selbstverletzenden Verhaltens (SVV). Die Borderline-Patienten wurden mit einer alters- und geschlechtsparallelisierten Kontrollgruppe stationär behandelter Patienten mit neurotischen und Anpassungs-Störungen verglichen. Sexueller und körperlicher Missbrauch sowie schwere emotionale Vernachlässigung waren bei den Borderline-Patienten signifikant häufiger zu finden als bei den Patienten der Kontrollgruppe. Die Kriterien einer PTBS erfüllten 67\% der Borderline-Patienten, hingegen nur 13\% der Vergleichsgruppe. Dissoziative Symptome waren in der Borderline-Gruppe signifikant stärker ausgeprägt. Anhand des Ausmaßes der dissoziativen Symptomatik und dem Vorliegen einer PTBS konnte selbstverletztendes Verhalten vorhergesagt werden (Spitzer et al. 2000).

Tabelle 2: Diagnostische Kriterien der Borderline-Persönlichkeitsstörung nach DSM-IV (APA 2003)

Symptommuster der Borderline-Persönlichkeitsstörung

- verzweifeltes Bemühen, tatsächliches oder vermutetes Verlassenwerden zu vermeiden

- ein Muster instabiler, aber intensiver zwischenmenschlicher Beziehungen, die durch einen Wechsel zwischen den Extremen der Idealisierung und Entwertung gekennzeichnet ist

- Identitätsstörung: ausgeprägte und andauernde Instabilität des Selbstbildes und der Selbstwahrnehmung

- Impulsivität in mindestens zwei potentiell selbstschädigenden Bereichen (Substanzmissbrauch, Geldausgaben, Sexualität, Kriminalität, risikohaftes Fahren, Fressanfälle)

- wiederkehrendes suizidales Verhalten, Suizidandrohungen oder selbstverletzendes Verhalten

- affektive Instabilität bei erheblichen Gefühlsschwankungen

- chronisches Gefühl der inneren Leere

- unangemessene, starke Wut oder Schwierigkeiten, Wut zu kontrollieren

- vorübergehende, belastungsabhängige paranoide Gedanken/Wahnvorstellungen oder schwere dissoziative Symptome

Die Borderline-Symptomatik wird von einigen Autoren als Ensemble von Copingstrategien zur Bewältigung von chronischen, nicht verarbeiteten posttraumatischen und dissoziativen Symptomen verstanden (Sachsse et al. 1997, Driessen et al. 2002). Aktuell wird diskutiert, inwiefern die BPS als traumaassoziierte Störung aufgefasst werden kann. Unter Berücksichtigung der allgemeinen Stressforschung und ähnlicher neuropsychologischer Defizite bei BPS und PTBS sprechen darüber hinaus erste psychoendokrinologische und bildgebende Studien für eine teilweise gemeinsame Pathogenese (Driessen et al. 2002). Insbesondere für die komplexe PTBS besteht bereits beim Vergleich der Diagnosekriterien eine erhebliche Überschneidung zur BPS. Eine aktuelle Multi-CenterStudie hat ergeben, dass eine Differenzierung der diagnostischen Cluster kPTBS und BPS nicht eindeutig möglich ist und beide Entitäten vermutlich die gleiche Patientengruppe zu erfassen versuchen (Sack et al. 2012). Auch das Muster an Komorbiditäten, welches von Angststörungen und depressiven, somatoformen, dissoziativen sowie substanzbezogenen Störungen geprägt ist, ist für kPTBS und BPS vergleichbar (Driessen et al. 2002, Sack et al. 2012). Vor diesem Hintergrund erheblicher Konvergenzen zwischen BPS und traumaassoziierten Störungen ist es offensichtlich, wie 
bedeutsam eine gründliche Auseinandersetzung mit posttraumatischen Zuständen für das Verständnis und die Therapie der Borderline-Persönlichkeitsstörung ist.

Das klinische Bild der BPS mit einer generellen emotionalen Vulnerabilität wurde bisher nur in wenigen psychophysiologischen Studien anhand hämodynamischer und autonomer Parameter fassbar gemacht und die Ergebnisse sind zum Teil inkonsistent. Einige Studien fanden überraschenderweise ein physiologisches Hypoarousal bei BPS-Patienten (Herpertz et al. 1999, Schmahl et al. 2004), während andere Arbeitsgruppen überschießende Reaktionsmuster auf Borderline-spezifische Stimuli nachweisen konnten (Lobbestael und Arntz 2010, Elices et al. 2012, Limberg et al. 2011). Der Vergleich von BPS- und PTBS-Patienten in ihrer Stressphysiologie (Schmahl et al. 2004) bzw. der Einfluss einer komorbiden PTBS auf die Reagibilität bei Borderline-Patienten (Limberg et al. 2011) wurde bisher nur in zwei Studien betrachtet. In der vorliegenden Studie soll deshalb der Einfluss einer ausgeprägten Borderline-Symptomatik auf die Stressphysiologie von (k)PTBS-Patientinnen untersucht werden, um diese Forschungslücke etwas zu füllen. Eine anhand psychometrischer Merkmale gebildete Patientinnen-Subgruppe "hoch Borderline" und deren Reaktionsmuster während der Stressbelastungsuntersuchung wird mit den restlichen (k)PTBS-Patientinnen des Ausgangskollektivs ohne ausgeprägte Borderline-Symptomatik verglichen (siehe Methodenteil Kapitel 3.8, Seite 34 ).

\subsubsection{Dissoziative Störungen (DS)}

Dissoziative Reaktionen dienen primär als Schutzmechanismen, um der Traumatisierung durch verminderte Reizwahrnehmung nicht in vollem Ausmaß ausgeliefert zu sein. Nach traumatischem Stresserleben sind häufig dissoziative anamnestische Zustände zu beobachten (Bremner et al. 1993, Zucker et al. 2006).). Jedoch können sie auch über die akute Belastungsreaktion hinaus persistieren und durch erschwerte Traumaverarbeitung und -integration zu einer Chronifizierung der Symptomatik führen. Typisch ist das Auftreten sehr unterschiedlicher und gegeneinander teilweise isolierter Erlebniszustände, mit dem Ziel ein Abgleiten in den traumatischen Erlebniszustand mit quälenden Erinnerungen auf jede nur mögliche Weise zu vermeiden. Es besteht eine gestörte Fähigkeit, Erinnerungen, Selbst- und Fremdwahrnehmung sowie Emotionen kohärent zu integrieren. Dissoziative Störungen sind durch Depersonalisation und Derealisation gekennzeichnet und können nach den Diagnosesystemen DSM-IV und ICD-10 differenziert klassifiziert werden (u.a. Dissoziative Amnesie (DA), Dissoziative Identitätsstörung (DIS); APA 2003, WHO 2006). Insbesondere langfristiger, gewalttätiger sexueller Missbrauch in der Kindheit und/oder Vernachlässigung stellen signifikante Prädiktoren für die Entwicklung einer dissoziativen Störung dar. Da das Bindungssystem der Kinder im Rahmen der Traumaerfahrungen stark beeinträchtigt wird, werden dissoziative Schutzmechanismen langfristig verankert (Lyons-Ruth 2008). Emotionale Abgestumpftheit und Amnesien können auch dissoziative Störungen andeuten. In Abgrenzung hierzu sind jedoch Intrusionen, Vermeidungsverhalten und Hyperarousal häufiger bei der PTBS vorhanden. Personen mit einer komplexen PTBS scheinen im Gegensatz zu Betroffenen mit einer einfachen, chronischen PTBS 
unter deutlich stärker ausgeprägten dissoziativen Episoden zu leiden (Zucker et al. 2006). Dissoziative Phänomene sind heute im DSM-IV (APA 2003) als charakteristische Symptome einer BorderlinePersönlichkeitsstörung beschrieben (Kriterium 9). Oftmals besteht eine Komorbidität einer dissoziativen Störung mit der BPS (Dammann und Overkamp 2004). Bei beiden Krankheitsbildern liegen zu einem hohen Prozentsatz frühe Traumatisierungen zugrunde, weshalb vielfach eine Klassifizierung als Traumafolgestörung diskutiert wird. Zur Unterscheidung zwischen den sich vielfach symptomatisch überschneidenden Störungsbildern BPS und DS kann man die Qualität der Affektregulation und der Beziehungsgestaltung betrachten. Während BPS-Patienten expressive, emotionale Schwankungen und intensive, aber instabile Beziehungsmuster aufweisen, behelfen sich hoch dissoziative Personen bei der Emotionsregulierung der Dissoziation und inneren Persönlichkeitswechseln und sind in der Lage langfristige Beziehungen zu führen (Overkamp und Helmke-Jaeger 2010). Es wird angenommen, dass Patienten in dissoziativen Zuständen kaum lernfähig sind und deshalb in diesen Zuständen auch nicht von Psychotherapie profitieren. Daher ist es entscheidend für das therapeutische Vorgehen bei Traumafolgestörungen, den Schweregrad einer vorliegenden komorbiden dissoziativen Störung abzuschätzen und ggf. diese als Hauptdiagnose und somit im Behandlungskonzept als vorrangig zu bewerten (Wöller und Kruse 2003).

\subsection{Traumazentrierte Therapie der kPTBS bzw. BPS auf der Station 9 des Asklepios Fachklinikums Göttingen}

Jede Form der psychotherapeutischen Hilfestellung erfordert als unverzichtbare Voraussetzung eine qualifizierte Diagnostik. Auf der spezialisierten Trauma-Station des Asklepios Fachklinikums Göttingen (AFG) erfolgt diese anhand strukturierter Interviews (DDIS, IPDE, I-kPTBS) und Fragebogendiagnostik mittels SCL-90-R, BDI, IES-R, FDS/DES, BSL sowie BPI (siehe Kapitel 3.4, Seite 22). Ziel der Diagnostikphase ist es, das Vorliegen einer psychischen Störung mit Krankheitswert zu überprüfen, diese im Kontext des Traumas, der Lebenssituation der betroffenen Person mit deren möglichen Risikofaktoren und Ressourcen psychodiagnostisch zu bewerten und abhängig vom Schweregrad die therapeutische Vorgehensweise festzulegen. Das therapeutische Konzept der Station 9 des AFG beruht auf dem Drei-Phasen-Modell nach Pierre Janet und Judith Herman (Reddemann und Sachsse 1997, 1998), arbeitet methodenintegrativ und umfasst, adaptiert an die konkrete Lebenssituation und Bedürfnisse der Patientin, Stabilisierungs- und Konfrontationsanteile. Als traumaspezifische Verfahren werden sowohl die psychodynamische Imaginative Traumatherapie (PITT, Reddemann 2004), Eye Movement Desensitization Reprocessing (EMDR, Shapiro 1995) sowie Elemente der Dialektisch-behavioralen Therapie (DBT, Linehan 1993) in eine Gesamtstrategie eingebettet. Behandelt werden ausschließlich Patientinnen mit dem Symptommuster einer (k)PTBS. Komorbiditäten wie beispielsweise BPS, SVV, Depressionen und/oder DS sind vielfach gegeben. Die Behandlung besteht aus den Phasen traumaspezifische Stabilisierung, Traumasynthese sowie Trauer und Neuorientierung, die mehrfach dynamisch durchlaufen werden (Müller und Sachsse 2009). Das traumazentrierte Behandlungskonzept zielt 
darauf ab, beide bei der kPTBS beeinträchtigten Distress-Systeme, das Panik- und das Furchtsystem, in die Therapie einzubeziehen. Eine Integration dieser Therapieverfahren im stationären Setting erwies sich als erfolgreich für die Behandlung der (k)PTBS sowie der BPS (Sachsse et al. 2006). 


\section{$2 \quad$ Zielsetzung und Hypothesen}

\subsection{Untersuchungsziele}

Zahlreiche Untersuchungen $\mathrm{zu}$ neurobiologischen Prozessen der PTBS weisen auf gestörte Stressverarbeitungsmechanismen hin, die wiederum zu einer gestörten Affekt- und Impulsreaktion der Patienten führen. Banale Alltagsbelastungen führen bei den Patienten zu Übererregungssymptomen (Hyperarousal) oder Dissoziation. Dass solche Reaktionsmuster psychisch traumatisierter Patienten u.a. eine veränderte sympathische und parasympathische Innervation des Herzens durch das autonome Nervensystem (ANS), etwa im Sinne einer verminderten Herzratenvariabilität, umfassen, ist zu vermuten. Einige Studien geben Hinweise darauf, dass bei der PTBS Veränderungen des sympathovagalen Gleichgewichtes mit vermindertem Vagotonus bzw. einer überaktivierten Sympathikusreaktion v.a. unter psychischer Stressbelastung vorliegen (Cohen et al. 1998, Sack et al. 2004, Hopper et al. 2006, Keary et al. 2009). Dass dies auch prognostisch relevant sein könnte, legen Befunde von Ladwig et al. (2008) nahe, die eine erhöhte Mortalität kardiologischer Patienten mit posttraumatischer Symptomatik fanden. Trotz vorliegender Einzelbefunde bleibt nach wie vor offen, ob und wenn ja in welchem Ausmaß eine (k)PTBS bzw. BPS die Innervation des HerzKreislaufsystems durch das ANS beeinflusst. Bei dieser vulnerablen Patientengruppe ist bisher kaum umfassend untersucht worden, welche hämodynamischen und autonomen Auswirkungen akuter psychischer Stress hat, und keine Studie prüfte bislang, ob sich unterschiedliche Effekte beim Einsatz eines kognitiven Stressors (z.B. Kopfrechnen) im Vergleich zu einer emotionalen Belastung (z.B. Babyschreien) nachweisen lassen. Die Fallzahlen der wenigen Studien bei PTBS-Patienten waren meist relativ gering und die differenzierte Beschreibung der hämodynamischen und autonomen Parameter sowie der zugrunde liegenden Regulationsmechanismen war nicht immer primäres Ziel der Untersuchung. Die vorliegende Studie schließt in die Beurteilung der psychophysiologischen Aktivierung sowohl alpha- und beta-adrenerge Reaktionsparameter als auch HRV-Parameter aus dem Zeit- sowie Frequenzbereich ein. Darüber hinaus soll eine Patientinnen-Subgruppe mit ausgeprägter Borderline-Symptomatik untersucht werden, um ggf. unterschiedliche Stressreagibilitätsmuster in Abgrenzung zu Patientinnen ohne oder fast ohne ausgeprägte BPS aufzudecken. Bisherige Ergebnisse zur Stressphysiologie bei Patienten mit BPS sind teilweise widersprüchlich und lediglich zwei Studien untersuchten die psychophysiologische Reaktivität von PTBS und BPS in Gegenüberstellung (Schmahl et al. 2004, Limberg et al. 2011). Generell wurde eine differenzierte Analyse von PTBSSubtypen bislang weitestgehend vernachlässigt.

An dieser Stelle wollen wir einen Forschungsbeitrag leisten und im Rahmen der vorliegenden, teilweise explorativen Studie folgenden Untersuchungszielen nachgehen: 
- Charakterisierung der psychophysiologischen Stressreagibilität bei Frauen mit (k)PTBS bzw. BPS unter Einwirkung verschiedener Stressoren und in Ruhe- bzw. Erholungssituationen

- Vergleich der Effekte zweier unterschiedlicher Stressoren (Babyschreien und Kopfrechentest)

- Klassifizierung des Patientinnenkollektivs in eine Subgruppe „hoch Borderline“ mit spezifischem Stressreagibilitätsmuster in Gegenüberstellung zum Rest des Ausgangskollektivs ohne ausgeprägte Borderline-Symptomatik.

\subsection{Hypothesen}

Basierend auf den o.g. Zielsetzungen wurden folgende Hypothesen herausgearbeitet:

1. Bei den PTBS-Patientinnen zeigen sich sowohl in Ruhe als auch unter Stressbelastung verschiedene pathophysiologische Modulationsmechanismen.

a) Die beiden spezifischen Stresstests rufen bei den Patientinnen im Vergleich zur

Ruhephase (Baseline) eine autonome kardiovaskuläre Aktivierung hervor, die sich in folgenden Werten zeigt:

- Zunahme des peripheren Widerstandes (TPRI)

- Steigerung des Cardiac Index (CI)

- Zunahme der LF-HRV sowie Abnahme der HF-HRV

- eingeschränkte SDNN.

b) Der emotionale Stressor „Babyschreien“ wird anders verarbeitet als der kognitive Stressor „Kopfrechnen“. Unter Babyschreien kommt es im Vergleich zum Kopfrechnen $\mathrm{zu}$ einer stärkeren Zunahme des TPRI mit einhergehender Blutdrucksteigerung. Der CI wird weniger gesteigert, die HF-HRV sowie die SDNN nehmen deutlicher ab. Somit wird beim Babyschreien eine andere Achse der sympathischen Aktivierung stimuliert.

c) In der Erholungsphase nach dem Babyschreien sind die erhobenen Erholungs-Werte dBP, CI, TPRI und RMSSD nicht signifikant niedriger als während des Babyschreiens, was auf eine verzögerte Rückbildungsfähigkeit nach emotionaler Stresseinwirkung hindeutet.

d) In der abschließenden meditativen Entspannungsphase erreichen die Patientinnen ähnliche Werte wie in Phase 1 (Baseline). 
2. Durch Simulation alläglicher Stressbelastungen ist bei Patientinnen mit (k)PTBS bzw. BPS eine subjektive Aktivierung im Vergleich zum subjektiven Stressempfinden in Ruhe sowie ein beobachtbares Auftreten vegetativer bzw. emotionaler Reaktionen induzierbar.

a) Das Babyschreien löst bei den Patientinnen ein stärkeres subjektives Stressempfinden als der Kopfrechentest aus.

b) Die beiden Stresstests rufen unterschiedliche emotionale, vegetative Symptomatiken hervor.

3. Eine durch spezifische psychometrische Merkmale abgrenzbare Patientinnen-Subgruppe „hoch Borderline“ zeigt eine gegenüber dem Restkollektiv unterschiedliche Ausprägung der Stressreaktion. 


\section{Methodik}

\subsection{Studiendesign}

Die Durchführung der vorliegenden diagnostischen Studie erfolgte im Zeitraum Oktober 2009 bis 2012 in der Klinik für Psychosomatische Medizin und Psychotherapie der Universitätsmedizin Göttingen (UMG). Nach Erhalt des zustimmenden Votums der zuständigen Ethik-Kommission der Medizinischen Fakultät der Universität zu Göttingen begann die konsekutive Patientinnenrekrutierung. Das Untersuchungsteam bestand aus den verantwortlichen Projektleitern Prof. Dr. C. HerrmannLingen und Prof. Dr. U. Sachsse, zwei Doktorandinnen sowie dem medizinischen Team der Station 9 des Asklepios Fachklinikums Göttingen (AFG).

Der Schwerpunkt dieser Dissertation liegt in der Auswertung der Stressreaktivität von Frauen mit (k)PTBS bzw. BPS. Dazu erfolgte nach stationärer Diagnostik und ausführlicher Aufklärung über die Studienziele sowie den Untersuchungsablauf eine psychophysiologische Stresstestung mittels Task Force Monitor (TFM). Diese einmalige Basisuntersuchung mit der nicht-invasiven TFM-Messung und den sie begleitenden Tests dauerte ca. eine Stunde.

Der Gruppe der Patientinnen mit (k)PTBS bzw. BPS wurde im Rahmen einer zweiten parallelen Doktorarbeit von G. Bornschein eine gesunde Kontrollgruppe vergleichend gegenübergestellt, welche unter identischen Untersuchungsbedingungen gemessen wurde. Somit ergeben sich zwangsläufig methodische Schnittstellen und bestimmte Datensätze sind Bestandteil beider Dissertationen. Eine Follow-Up-Untersuchung der Patientinnen ist in Vorbereitung.

\subsection{Patientinnenrekrutierung}

Das Untersuchungskollektiv umfasste 54 stationäre Patientinnen, die von ihren niedergelassenen betreuenden Psychotherapeuten mit dem klinischen Verdacht auf eine (k)PTBS bzw. BPS auf die spezialisierte Trauma-Station des AFG überwiesen wurden. Die rekrutierten Teilnehmerinnen sind weiblichen Geschlechts und im Alter zwischen 20 und 56 Jahren. Die Patientinnen befanden sich entweder zur ein- bzw. zweiwöchigen Diagnostikphase oder bereits zum ersten Therapieintervall in stationärer Behandlung. Die auszuwertenden Interviews und Fragebogendaten sind Bestandteil der Routinediagnostik und Qualitätssicherung des AFG, sodass für die Patientinnen hierdurch keine zusätzliche Belastung entstand. Im Rahmen der Studie erfolgte keine Einflussnahme auf die laufende Behandlung oder Diagnostik der Patientinnen.

\subsubsection{Einschlusskriterien}

Eingeschlossen wurden konsekutiv stationär in der Klinik für Psychotherapie des AFG befindliche Patientinnen mit Verdacht auf eine (k)PTBS bzw. BPS, vorausgesetzt, sie stimmten nach erfolgter 
Aufklärung der Untersuchung durch Unterschreiben der Einverständniserklärung zu. Weitere Einschlusskriterien waren ein Alter zwischen 18 und 59 Jahren (im Interesse einer Vermeidung von altersbedingten Veränderungen der zu bestimmenden Herzfunktionsparameter), ausreichende kognitive Fähigkeiten und Beherrschung der deutschen Sprache.

\subsubsection{Ausschlusskriterien}

Als Ausschlusskriterien galten eine fehlende schriftliche Einwilligung in die Studienteilnahme sowie ein Alter jünger als 18 Jahre bzw. älter als 59 Jahre. Auch bekannte schwere Herzerkrankungen wie beispielsweise hochgradige Herzrhythmusstörungen (v.a. Vorhofflimmern) oder schwere Herzinsuffizienz sowie eine diabetische Polyneuropathie führten zum Ausschluss von der Studienteilnahme. Schwere Herzerkrankungen sind auch eine relative Kontraindikation für physiologisch belastende Traumaexpositionen.

Medikamente, die die Herzaktivität und insbesondere den Vagotonus stark beeinträchtigen, wurden dokumentiert, führten jedoch nicht zum Studienausschluss. Die Befunde dieser Patientinnen wurden in der parallel durchgeführten Studie von G. Bornschein gezielt analysiert.

\subsection{Aufbau und Ablauf der Studie}

Die Studie bestand aus zwei aufeinander folgenden Untersuchungsabschnitten:

Im ersten Untersuchungsabschnitt wurden anhand der routinemäßig durchgeführten strukturierten Interviews und Fragebogendiagnostik (siehe Kapitel 3.4, Seite 22) auf Station 9 des AFG bei den Patientinnen präzise DSM-IV-Diagnosen gestellt und eine psychometrische Charakterisierung durchgeführt. Zusätzlich wurden soziodemographische Daten mit Hilfe eines selbst erstellten, allgemeinen Fragebogens erhoben.

Im zweiten Untersuchungsabschnitt wurde eine psychophysiologische Messung mit einem nichtinvasiven Monitoringsystem zur Erfassung hämodynamischer sowie autonomer Parameter, dem Task Force Monitor, durchgeführt. Diese erfolgte sowohl in Ruhe als auch unter psychischer Stressbelastung. Ausgewählt wurden zwei alltägliche Stressoren, denen niemand im Alltag auf Dauer ausweichen kann. Die Stressbelastung wurde dabei durch zwei aufeinander folgende Tests hervorgerufen: ein als Stresstestverfahren weit verbreiteter Rechen-Stresstest (Kopfrechenaufgabe unter Zeitdruck), der als überwiegend kognitiv-mentale Belastung verstanden werden kann, und eine Exposition gegen Babyschreien (mittels Wiedergabe einer standardisierten Tonaufnahme), die als überwiegend emotionale Belastung verstanden werden kann. Daran anschließend erfolgte eine Ruhephase, in der den Versuchsteilnehmerinnen eine entspannende Musik (Ludwig van Beethoven: Klaviersonate Nr. 14, „Mondscheinsonate“) vorgespielt wurde.

Im Rahmen einer Follow-Up-Untersuchung sollen die Patientinnen nach einigen Behandlungsintervallen bzw. nach Abschluss der Psychotherapie nach ca. 2 bis 3 Jahren erneut mit der gleichen Methode untersucht werden. Der gesamte zeitliche Verlaufsplan der einzelnen 
Untersuchungsabschnitte ist in Abbildung 2 (Seite 22) dargestellt.

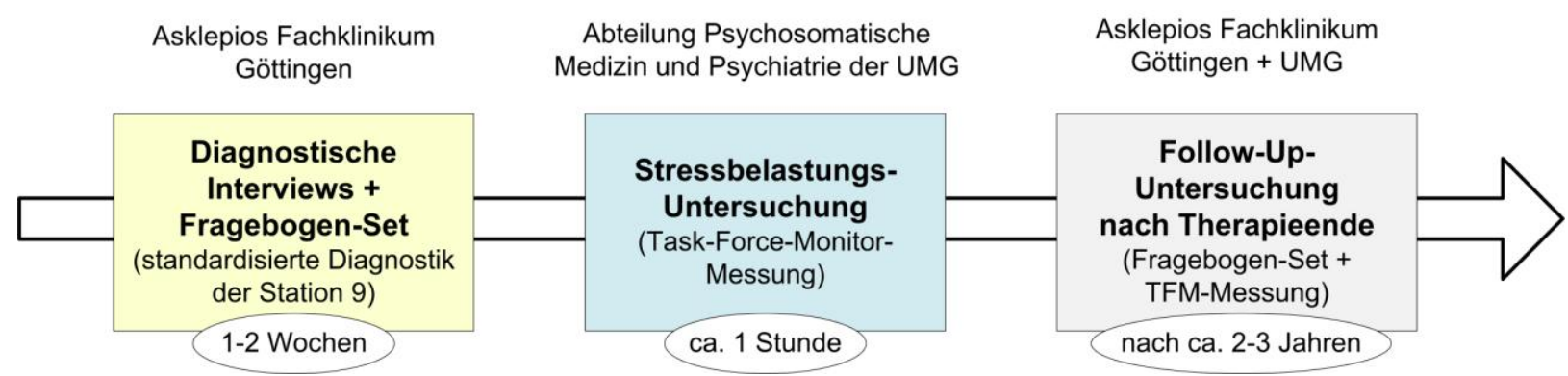

Abbildung 2: Studienablauf: einzelne Untersuchungsabschnitte für Patientinnen

\subsection{Diagnostische Interviews und Fragebogen-Mappe}

Die auf Station 9 des AFG routinemäßig durchgeführten strukturierten Interviews (DDIS, IPDE, IkPTBS) und Fragebogendiagnostik (SCL-90-R, BDI, FDS, IES-R, BSL, BPI) dienten der standardisierten DSM-IV-Diagnosestellung bei den Patientinnen sowie der detaillierten psychometrischen Charakterisierung. Darüber hinaus füllten die Patientinnen vor Durchführung der Stressbelastungsuntersuchung einen allgemeinen Fragebogen zur einheitlichen Erhebung soziodemographischer Daten aus.

\subsubsection{Dissociative Disorders Interview Schedule (DDIS)}

Beim Dissociative Disorders Interview Schedule (DDIS) von Ross et al. (1989) handelt es sich um einen 131 Items umfassenden, hochstrukturierten diagnostischen Interviewleitfaden, der sich in der aktuellen Fassung an den DSM-IV-Diagnosen für dissoziative Störungen, Somatisierungsstörung, Major-Depression, Schizophrenie und BPS orientiert (APA 2003). Darüber hinaus erfasst der DDIS bei Patienten mit dissoziativer Identitätsstörung (DIS) häufig anzutreffende Symptome und Erfahrungen wie Substanzabusus, Gewalterfahrungen in der Kindheit, das Auftreten von SchneiderSymptomen ersten Ranges und Trancephänomene sowie sekundäre Anzeichen einer DIS (z.B. außersinnliche Wahrnehmungen, Erinnerungslücken, Hören von Stimmen). Die Erfassung von Traumatisierungserfahrungen wird auf der Trauma-Station des AFG dabei erst nach ausreichender Stabilität zu Beginn der zweiten Therapiephase vorgenommen, um überfordernden Intrusionen und Flashbacks vorzubeugen. Der Fragentypus des Interviews ist geschlossen. Die Interviewdauer kann von 30 bis 90 Minuten variieren. Die deutsche Fassung, der strukturierte Interviewleitfaden zur Diagnostik dissoziativer Störungen (SIDDS), wurde von Frör und Overkamp validiert (Frör 2000, Overkamp 2005).

Der DDIS verfügt über gut getestete Reliabilität und klinische Validität sowohl in ambulantem und stationärem Setting mit sehr hoher Sensitivität und Spezifität für dissoziative Persönlichkeitsstörung als auch in der Differentialdiagnose zu anderen dissoziativen Störungen (Ross et al. 1990). 


\subsubsection{International Personality Disorder Examination (IPDE)}

Mit der International Personality Disorder Examination (IPDE; Loranger 1999) können Persönlichkeitsstörungen nach den Kriterien der ICD-10 (WHO 2006) sowie des DSM-IV Klassifikationssystems (APA 2003) erfasst werden. Es handelt sich um eine Erweiterung der Personality Disorder Examination (PDE) von Loranger (1988). Die Untersuchung gliedert sich in einen Screening-Fragebogen und ein anschließendes semistrukturiertes Interview. Der von den Patientinnen auszufüllende IPDE-Fragebogen beinhaltet 77 mit „richtig“ oder „falsch“ zu beantwortende Selbstaussagen. Die Beantwortung der Fragen dauert in der Regel ca. 15 Minuten und dient dem Ausschluss von Personen ohne Persönlichkeitsstörung. Ergibt sich aus dem ScreeningFragebogen ein auffälliger Wert, folgt das IPDE-Interview, welches insgesamt 67 (ICD-10-Modul, WHO 2006) bzw. 99 (DSM-IV-Modul, APA 2003) Fragen aus den sechs Bereichen Arbeit, Selbst, zwischenmenschliche Beziehungen, Affekte, Realitätsbeurteilung und Impulskontrolle umfasst. Ein Verhalten muss in der Regel mindestens fünf Jahre lang bestanden haben, um als Persönlichkeitsmerkmal angesehen $\mathrm{zu}$ werden. Das IPDE-Interview erlaubt die Diagnose unterschiedlicher Persönlichkeitsstörungen (nach ICD-10, WHO 2006: paranoid, schizoid, dissozial, emotional instabil (vom impulsiven Typ bzw. vom Borderline-Typ), histrionisch, anankastisch, ängstlich, abhängig und unspezifisch). Aufgrund zahlreicher Übersetzungen ist das Instrument weltweit einsetzbar. In verschiedenen Studien wurde die Validität und Reliabilität dieses Verfahrens überprüft (Loranger et al. 1994).

\subsubsection{Das Interview zur Diagnostik der komplexen posttraumatischen Belastungsstörung (I-kPTBS)}

Aufgrund der Problematik, das sehr breite und unspezifische Symptomspektrum einer komplexen posttraumatischen Belastungsstörung schwer erfassen zu können, entwickelten Pelcovitz et al. (1997) ein spezielles diagnostisches Interview, das Structured Clinical Interview for Disorder of Extreme Stress (SIDES). Darauf basiert die deutsche Fassung, das Interview zur komplexen posttraumatischen Belastungsstörung (I-kPTBS) von Sack und Hofmann (2001), das inzwischen validiert ist (BoroskeLeiner et al. 2008). Das Interview erfasst Symptome aus den Bereichen Störungen der Affekt- und Impulskontrolle inklusive selbstschädigendem Verhalten, Störungen der Wahrnehmung und des Bewusstseins, Störungen der Selbstwahrnehmung, Beziehungsstörungen, Somatisierungen und Veränderungen von zentralen Lebenseinstellungen und Zukunftsperspektiven.

\subsubsection{Symptom-Check-Liste (SCL-90-R)}

Die Symptom-Check-Liste (SCL-90-R) nach Derogatis et al. (1973) ist ein weit verbreiteter Selbstbeurteilungs-Fragebogen, welcher subjektiv empfundene Beeinträchtigung durch körperliche und psychische Symptome in einem Zeitfenster von sieben Tagen misst. Die deutsche Version wurde 1995 von Franke publiziert (Franke 1995). Durch seine fünfstufig zu gewichtenden 90 Items werden 
neun verschiedene Krankheitsdimensionen betrachtet, wobei durch einen Gesamtindex unter anderem zuverlässig zwischen Kranken und Gesunden unterschieden werden kann. Die neun Skalen beschreiben die Bereiche Somatisierung, Zwanghaftigkeit, Unsicherheit im Sozialkontakt, Depressivität, Ängstlichkeit, Aggressivität/Feindseligkeit, phobische Angst, paranoides Denken und Psychotizismus. Drei globale Kennwerte geben Auskunft über das Antwortverhalten bei allen Items. Dabei werden die grundsätzliche psychische Belastung, die Intensität der Antworten und die Anzahl der Symptome, bei denen eine Belastung vorliegt, gemessen. Die Durchführungszeit beträgt 10 bis 15 Minuten. Das Selbstbeurteilungsinventar kann sowohl als Screening-als auch als Outcome-Instrument eingesetzt werden, ist änderungssensitiv und weist eine gute Reliabilität auf (Franke 2001).

\subsubsection{Beck-Depressions-Inventar (BDI)}

Das Beck-Depressions-Inventar (BDI) von Beck et al. (1961) ist ein gut erforschter, weit verbreiteter Selbstbeurteilungs-Fragebogen, der auf 21 Items depressive Symptomatik abbildet und in ihrem Schweregrad unterscheidbar macht. Die von 0 bis 3 gekennzeichneten Items beziehen sich auf Symptome der Depression wie Hoffnungslosigkeit oder Reizbarkeit, Kognitionen wie Schuldgefühle oder das Gefühl, bestraft zu werden, sowie auch auf physische Symptome wie Müdigkeit, Gewichtsoder Libidoverlust. Das Instrument besitzt eine ausreichende interne und externe Konstruktvalidität, sodass es zur Differential- und Verlaufsbeurteilung von Depression sowie zur Evaluation der Wirksamkeit antidepressiver Therapie einsetzbar ist (Richter et al. 1998).

\subsubsection{Fragebogen zu Dissoziativen Symptomen (FDS)}

Bei dem Fragebogen zu dissoziativen Symptomen (FDS; Freyberger et al. 1999) handelt es sich um die deutsche Adaptation der Dissociative Experience Scale (DES) von Bernstein und Putnam (1986). Während die Originalskala (DES) mit 28 Items die Symptombereiche dissoziative Amnesie, Depersonalisation und Derealisation erfasst, bildet der FDS auf 16 zusätzlichen Items auch Konversionssymptome sowie somatoforme dissoziative Erfahrungen ab. Mit dieser Erweiterung ist der FDS auch an die ICD-10-Klassifikation dissoziativer Störungen angepasst (WHO 2006). Auf einer weiteren Subskala werden dissoziative Alltagserfahrungen (Absorptionserleben) dokumentiert. Der FDS basiert auf der Methode der Selbstbeurteilung und seine Bearbeitungszeit beträgt 5 bis 15 Minuten. Sowohl die DES als auch der FDS wurden umfassend validiert und weisen sehr gute TestGüte-Kriterien auf (van Ijzendoorn und Schuengel 1996).

\subsubsection{Impact of Event Scale (IES-R)}

Die revidierte Version der Impact of Event Scale (IES-R) von Weiss und Marmer (1996) dient als ein Selbstbeurteilungsmaß zur Erfassung posttraumatischer Belastungsreaktionen. Die Items der IES wurden ursprünglich aus dem theoretischen Modell traumatischer Stressfolgen (Horowitz et al. 1979) abgebildet. Die drei Subskalen Intrusion, Vermeidung und Übererregung erfassen typische Formen 
individueller Reaktionen bzw. Symptome auf extrem belastende Ereignisse. Zwei Items beziehen sich zusätzlich auf den Störungsbereich der emotionalen Taubheit (Numbing). Eine Schätzformel erlaubt aus den drei Subskalen das Vorliegen einer PTBS-Diagnose abzuschätzen. Die Screening-Skala für posttraumatische Symptome wird auch im Rahmen von Verlaufskontrollen eingesetzt. Reliabilität, Validität und diagnostische Sensitivität erwiesen sich in Untersuchungen als zufriedenstellend (Maercker und Schützwohl 1998).

\subsubsection{Borderline-Persönlichkeits-Inventar (BPI)}

Bei dem Borderline-Persönlichkeits-Inventar (BPI; Leichsenring 1997) handelt es sich um ein aus 53 Items bestehendes Selbstbeurteilungs-Instrument, welches auf Kernbergs Konzept der BorderlinePersönlichkeitsorganisation (Kernberg 1967) basiert. Das BPI umfasst vier faktorenanalytisch konstruierte Skalen zur Erfassung von Entfremdungserlebnissen und Identitäts-Diffusion, primitiven Abwehrmechanismen und Objektbeziehungen, mangelhafter Realitätsprüfung sowie von Angst vor Nähe. Darüber hinaus wird ein Cut-Off-Wert basierend auf 20 Items (Cut-20) zur Unterscheidung der Borderline-Persönlichkeitsstörung von Neurosen einerseits und Schizophrenien andererseits ermittelt. Bei einem Kriterium Cut-20 $\geq 10$ kann eine BPS als sehr wahrscheinlich angenommen werden, während ein Cut-20 $\leq 10$ als ein mögliches Ausschlusskriterium einer BPS-Diagnose verwendet wird. Die Durchführungszeit des BPI beträgt ca. 20 Minuten. Nach den Ergebnissen zahlreicher Studien ist sowohl interne Konsistenz und die Test-Retest-Reliabilität als auch die Sensitivität, Spezifität und Validität des BPI zufriedenstellend (Leichsenring und Chabrol 2006).

\subsubsection{Borderline-Symptom-Liste (BSL)}

Die Borderline-Symptom-Liste (BSL) wurde von Bohus et al. (2001) zur Quantifizierung subjektiv erlebter intrapsychischer Belastung und Beeinträchtigung von Borderline-Patienten innerhalb der letzten sieben Tage entwickelt. Die BSL basiert auf den Kriterien des DSM-IV (APA 2003), dem Diagnostischen Interview für Borderline-Störungen (DIB, Gunderson et al. 1981), Expertenmeinungen und Patientenaussagen. Dieses etablierte Selbstbeurteilungsinstrument setzt sich aus insgesamt 95 Items zusammen, die anhand einer fünfstufigen Likertskala beurteilt werden. 83 dieser Items werden den sieben Subskalen Selbstwahrnehmung, Affektregulation, Autoaggression, Dysphorie, soziale Isolation, Intrusionen sowie Feindseligkeit zugeordnet. Zusätzlich wird mittels einer visuellen Analogskala die aktuelle psychische Befindlichkeit erhoben. Auf einer BSL-Ergänzungsskala (11 Items) kann das Ausmaß dysfunktionaler Verhaltensweisen erfasst werden. Darüber hinaus kann ein Gesamtfaktor gebildet werden, in den alle 95 Items eingehen. Die Auswertung erfolgt anhand von Prozenträngen, welche die relative Stellung der einzelnen Testperson angeben, die diese bezüglich der Symptomausprägung in einer Normierungsgruppe bestehend aus 308 Borderline-Patientinnen einnimmt. Analysen zu den Testgütekriterien erbrachten gute bis sehr gute Ergebnisse für die innere Konsistenz, Test-Retest-Reliabilität sowie Konstruktvalidität (Bohus et al. 2007). 


\subsubsection{Allgemeiner Fragebogen zur Erfassung soziodemographischer Daten und Risikofaktoren}

Um ergänzende Angaben zu den Untersuchungspersonen zu erhalten, eine bessere Vergleichbarkeit zu gewährleisten und mögliche Confounder zu erfassen, wurden zusätzlich folgende Daten erhoben: Alter, Größe, Gewicht, Familienstand, Zusammenleben mit Partner (ja/nein), Anzahl eigener Kinder, körperliche Aktivität (Stunden Sport pro Woche), Alkoholgenuss (drinks per week), Raucherstatus (packyears), Ausbildungsstand, Beschäftigungsverhältnis, aktuelle Medikamentenanamnese, Vorerkrankungen und Stressempfinden der Untersuchungsperson zum Zeitpunkt der Messung. Dieser Fragebogen ist der Arbeit im Anhang in einem halb so großen Format beigefügt.

\subsection{Erfassung klinischer Daten}

Weitere klinische Daten wie psychiatrische Haupt- und Nebendiagnosen, Suchtverhalten, Vorerkrankungen, Phase des Klinikaufenthalts (Diagnostikphase oder Therapieintervall), Art der Traumatisierung sowie Medikamenteneinnahme wurden den Krankenakten entnommen.

\subsection{Die Stressbelastungsuntersuchung}

Die Untersuchung fand in einem separaten Raum der Klinik für Psychosomatische Medizin und Psychotherapie der UMG mit angenehmer Atmosphäre statt. Die Teilnehmerinnen saßen während der TFM-Messung in einem bequemen Sessel. Es wurde darauf hingewiesen, sich während der gesamten Messung möglichst wenig zu bewegen, um Verfälschungen der Messwerte und ein Ablösen der Elektroden zu vermeiden. Das Angebot, die Untersuchung jederzeit bei Unannehmlichkeiten bzw. zu starkem Stressempfinden abbrechen zu können, wurde den Patientinnen unterbreitet. Parallel zu den einzelnen Phasen der Untersuchung wurde mittels einer Analogskala das subjektive Stressempfinden der Patientinnen erfragt. Das für alle teilnehmenden Patientinnen einheitliche Versuchsprotokoll ist in Abbildung 3 (Seite 28) dargestellt.

\subsubsection{Die Stresstests „Rechnen“ und „Babyschreien“}

Ein besonderes Element der Studie ist der Einsatz zweier unterschiedlicher Alltagsstressoren, mit denen versucht wurde, bei den Teilnehmerinnen verschiedene Formen alltäglichen emotionalen Stresserlebens auszulösen. Die Patientinnen wurden vor Beginn der Messungen darauf hingewiesen, dass sie bei zu großem Stressempfinden die Stressbelastungstests jederzeit abbrechen können. Durch die vorab auf Station stattgefundene Aufklärung waren die Patientinnen gut vorbereitet und nutzten teilweise individuelle Copingmechanismen. Verhalten, Empfindungen sowie emotionale bzw. vegetative Begleitsymptomatiken während der Messung wurden notiert. Eine eventuelle kurzfristige psychische Belastung der Patientinnen auch nach Abschluss der Tests wurde durch ein anschließendes Debriefing auf Station minimiert und konnte ggf. im Rahmen des therapeutischen Prozesses aufgefangen werden. Vor und zwischen den Stresstests wurde jeweils eine fünfminütige Ruhepause 
eingeschoben, in der das subjektive Stresserleben während der Stresstest-Phasen mittels Analogskala erfragt wurde.

Als kognitiver Stressor diente der allgemein übliche und in zahlreichen psychophysiologischen Studien eingesetzte Mental Arithmetic Stress Test (Kop et al. 2004, Lache 2006). Hierbei handelt es sich um einen fünfminütigen fortlaufenden Subtraktionstest, bei dem die Patientin von der vierstelligen Zahl 3505 fortlaufend die Zahl 13 subtrahieren sollte. Die Versuchsteilnehmerinnen wurden darauf hingewiesen, dass es sowohl auf Schnelligkeit als auch auf Fehlerfreiheit ankomme, wodurch Zeit- und Leistungsdruck simuliert wurden. Entsprechend der unterschiedlichen kognitiven Fähigkeiten der Untersuchungspersonen wurde individuell verschieden stark Druck aufgebaut oder auch eher zum Rechnen ermutigt und unterstützt. Ziel war es jedoch stets eine Stresssituation auszulösen.

Sozialer, emotionaler Alltagsstress sollte mittels Babyschreien hervorgerufen werden. Die Exposition erfolgte mit Hilfe einer standardisierten, fünfminütigen Tonbandaufnahme über Kopfhörer. Es wurde noch einmal darauf hingewiesen, dass das Babyschreien bei zu großer Belastung abgebrochen werden kann. Diese Form der Stresstestung wurde bisher noch nicht im Rahmen von PTBS-Studien eingesetzt und wurde als neuer, leicht durchzuführender emotionaler Stresstest bei dieser Patientinnengruppe erprobt. Unter der Annahme, dass Babyschreien bei diesem vulnerablen Untersuchungskollektiv einen potenten Stimulus darstellt, um eine emotionale Stressituation auszulösen, wurde dieser Stresstest in Gegenüberstellung zum Rechentest als eher kognitiver Stimulus gewählt.

\subsubsection{Ruhephasen und Entspannungsmusik}

Am Anfang der Messung stand eine fünfminütige Ruhephase, in der sich die Patientinnen zunächst an die ungewohnte Situation adaptieren konnten. Die Untersuchungspersonen sollten sich während der Ruhepausen möglichst entspannen und ruhig sitzen. Nach den Stresstests konnte in den Ruhephasen jeweils die Abnahme des Erregungsniveaus ermittelt werden. In den Ruhephasen 2 und 3 nach Stressanforderung erfolgte jeweils einheitlich in der dritten Minute ein manueller Wechsel der Fingermanschetten, um einem unangenehmen Kribbelgefühl im Finger vorzubeugen.

Zum Abschluss der Untersuchung erfolgte eine Ruhephase mit entspannender Musik. Es wurde die Klaviersonate Nr. 14 von Ludwig van Beethoven, auch bekannt als Mondscheinsonate, gewählt. Primäres Ziel sollte eine Entspannung sein. Wurde die Musik jedoch als nicht entspannend sondern als unangenehm bzw. emotional erregend empfunden, wurde dies dokumentiert. 
Anbringen der Messvorrichtung und Kalibrierung

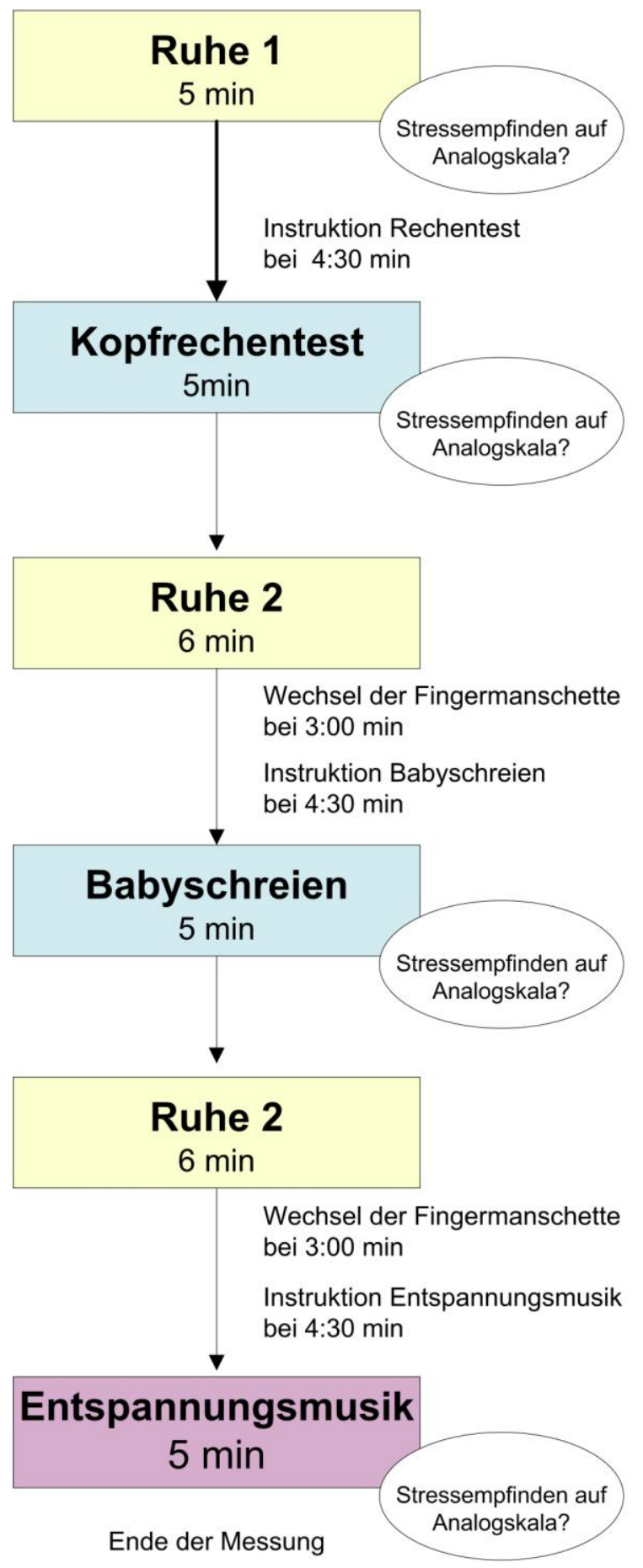

Abbildung 3: Messprotokoll der Stressbelastungsuntersuchung

\subsubsection{Analogskala subjektives Stressempfinden und beobachtbare emotionale bzw. vegetative} Reaktionen

Mit Hilfe einer Analogskala von 0 bis 10 (Subject Units of Discomfort SUD, Wolpe 1958) wurde das subjektive Stressempfinden der Patientinnen zu Beginn der Messung sowie unmittelbar nach dem 
Rechenstresstest, dem Babyschreien und der Entspannungsmusik erfragt. Hierbei entsprach „0“ gar keinem Stress und „10“ dem schlimmsten Stress, den sich die Versuchsteilnehmerin vorstellen konnte. Während der Stressbelastungsuntersuchung zeigten einige Patientinnen beobachtbare emotionale bzw. vegetative Reaktionen. Dabei wurden folgende Reaktionsformen kategorial dokumentiert:

- schwere Atmung: erhöhte Atemfrequenz bzw. -tiefe

- Erregungszustände: Gänsehaut, Schwitzen, Herzklopfen, Muskelzucken

- Weinen bzw. Tränen in den Augen

- Nutzen von Copingmechanismen: Coolpack, Stachelball, „Herunteratmen“

- Numbing-Verhalten: starrer Blick, verzögerte Reaktion auf Ansprechen.

\subsection{Der Task Force Monitor (Kardiovegetative Funktionsmessung)}

Der Task Force Monitor (TFM) der Firma CNSystems Medizintechnik GmbH (Graz, Österreich) ist ein rechnergestütztes Messsystem zum nicht-invasiven Monitoring hämodynamischer und autonomer Parameter. Hierfür bietet das Gerät neben einem Zwei-Kanal-EKG, oszillometrischer Blutdruckmessung und nicht-invasiver kontinuierlicher Blutdruckmessung auch die Möglichkeit der Impedanzkardiographie (IKG). Mit Hilfe der zugehörigen Software stehen folgende Analysemöglichkeiten zur Verfügung: nicht-invasive Beat-to-beat-Blutdruckmessung mit automatischer Korrektur der Absolutwerte durch das oszillometrische Blutdruckmessgerät, Echtzeitanalyse von Sympathikotonus und Vagotonus aus Herzraten- und Blutdruckvariabilität, Barorezeptorsensitivität, nicht-invasive Schlagvolumen- und Herzzeitvolumenbestimmung für jeden Herzschlag, Bestimmung des peripheren Gefäßwiderstands, Ermittlung der positiven Inotropie des Herzens sowie der Präejektionsperiode (Gratze et al. 1998).

Zusammenfassend liefert der TFM vier unterschiedliche Messsignale vom Patienten:

- fingerplethysmographische Blutdruckwerte (kontinuierlich, beat-to-beat)

- oszillometrische Blutdruckwerte (absolut)

- EKG-Potentiale

- Impedanz-Potentiale.

Optional kann eine Pulsoxymetrie an den Task Force Monitor angeschlossen werden.

Abgeleitet aus den Beat-to-beat-Daten der kontinuierlichen Blutdruckmessung sowie des Elektrokardiogramms können autonome Parameter wie die Herzraten- und Blutdruckvariabilität sowie die Barorezeptorsensitivität berechnet werden (Gratze et al. 1998).

Bei dem TFM handelt sich um ein gut evaluiertes (Fortin et al. 2001), durch den TÜV Österreich 2000 zertifiziertes (CE 0408) und seit 2002 auch durch die amerikanische FDA anerkanntes physiologisches Monitoringsystem für hämodynamische Parameter. 


\subsubsection{Blutdruckmessung und EKG}

Bei der nicht-invasiv kontinuierlichen Beat-to-beat-Blutdruckmessung wird der arterielle Fingerblutdruck mit Hilfe des fingerplethysmographischen Prinzips (Vascular Unloading-Technik) ermittelt (Penaz et al. 1976). Hiermit können relative Veränderungen des Blutdruckes kontinuierlich erfasst werden, welche regelmäßig an die absoluten Blutdruckwerte aus der oszillometrischen Blutdruckmessung am kontralateralen Oberarm angepasst werden (Fortin et al. 2006a). Das Elektrokardiogramm ist ein Zwei-Kanal-EKG mit vier Spotelektroden. Das EKG-Signal wird mit $1000 \mathrm{~Hz}$ abgetastet, wodurch eine sehr exakte Ermittlung der RR-Intervalle möglich ist.

\subsubsection{Impedanzkardiographie}

Im Rahmen der transthorakalen Impedanzkardiographie (IKG) wird ausgehend von Impedanzänderungen auf Veränderungen des intrathorakalen Blutvolumens geschlossen (Fortin et al. 2006b). Hieraus können das Beat-to-beat-Schlagvolumen (SV) und das Herzzeitvolumen (HZV) bestimmt werden.

\subsubsection{Herzratenvariabilität (HRV)}

Zur Berechnung der HRV stehen sowohl Analysen im Zeit- (Time-Domain) als auch im Frequenzbereich (Frequency-Domain) zur Verfügung.

Zeitbereich: Die Analyse umfasst die Zeitdauer aufeinander folgender RR-Intervalle und erhebt u.a. folgende Indizes (Hilz und Dütsch 2006):

- SDNN: Standardabweichung aller RR-Intervalle.

- RMSSD: Root Mean Square of Successive Differences.

Die SDNN reflektiert die Gesamtvariabilität der HRV, die sowohl sympathische als auch vagale Einflüsse auf die Herzfrequenz widerspiegelt.

Hinter RMSSD verbirgt sich die mittlere Differenz aufeinander folgender Zyklusdauern, welche v.a. hochfrequente Änderungen erfasst und daher ein selektives Maß für die kardiale tonische Vagusaktivität darstellt. Zur Berechnung der RMSSD muss die Quadratwurzel aus der Summe quadrierter Differenzen aufeinander folgender RR-Intervalle (RRI) genommen werden.

Frequenzbereich: Zur Bestimmung von Sympathikotonus und Vagotonus aus Herzraten- und Blutdruckvariabilität dient die sogenannte Power-Spektralanalyse (Task Force 1996). Im Rahmen einer Frequenzdichteanalyse werden die Werte des RR-Intervalls (RRI) und des Beat-to-beatBlutdrucksignals (systolisch und/oder diastolisch) aus dem Zeitbereich in den Frequenzbereich transformiert. Die Software des TFM verwendet dazu den Algorithmus der Adaptiven Autoregressiven Parameter (AAR; Schlögl et al. 1997), dabei entsprechen die Spektralanteile jeweils dem prozentualen Anteil eines bestimmten Frequenzbandes an der Gesamt-Variabilität.

Das Messspektrum für die HRV lässt sich in drei Frequenzbänder aufteilen: 
- High Frequency HF: $0.15-0.4 \mathrm{~Hz}$

- Low Frequency LF: $0.04-0.15 \mathrm{~Hz}$

- Very Low Frequency VLF: $\leq 0.04 \mathrm{~Hz}$.

Die über längere Messzeiträume dominierenden langsamen Oszillationen (VLF) repräsentieren humoral bedingte Sinusknotenmodulationen sowie thermoregulatorische, zirkadiane und vasomotorische Prozesse. Während kurzer Messzeiträume lassen sich hochfrequente und niederfrequente Rhythmen unterscheiden. Der Hochfrequenzbereich ist Ausdruck der respiratorischen Sinusarrhythmie (RSA), die eine vagal vermittelte permanente Anpassung der Herzfrequenz an den Atemzyklus darstellt. Die HF-HRV korreliert daher mit dem Vagotonus und gilt als Marker für die vagale kardiale Modulation. Die niederfrequenten Anteile der HRV sind sympathisch vermittelte Oszillationen. Jedoch unterliegen die LF-Schwankungen über den Baroreflex auch einem vagalen Einfluss. Somit gibt die LF-HRV Auskunft sowohl über den sympathischen als auch den vagalen Tonus. Das Verhältnis LF/HF, die sogenannte HRV-Ratio, gilt als Marker der sympathovagalen Balance. Eine Erhöhung der HRV-Ratio kann durch eine verminderte vagale oder eine erhöhte sympathische Aktivität bedingt sein. Der sympathische Tonus wird auch durch das Niederfrequenzband der Blutdruckvariabilität (LF-Band $0.04-0.15 \mathrm{~Hz}$ ) repräsentiert (Task Force 1996).

\subsubsection{Barorezeptorsensitivität (BRS)}

Die BRS ermöglicht eine Beurteilung der Blutdrucksteuerung durch das ANS und wird mit Hilfe der Sequenzmethode (Parati et al. 1988) ermittelt. Eine BRS-Sequenz ergibt sich hierbei aus dem Zusammenspiel von systolischen Blutdruckschwankungen und darauf folgender Pulsveränderung. Ein Barorezeptor-Event liegt vor, wenn bei drei aufeinander folgenden Herzschlägen eine Veränderung des systolischen Blutdrucks (sBP) um mindestens $1 \mathrm{mmHg}$ in dieselbe Richtung detektiert wird und auch das RR-Intervall (RRI) eine Verlängerung bzw. Verkürzung um je mindestens 4 ms aufweist.

\subsubsection{Auswahl der TFM-Parameter für die Auswertung der Stressreagibilität}

Zusammenfassend werden folgende hämodynamische und autonome Werte im Rahmen der vorliegenden Studie in die Auswertung eingeschlossen (Abbildung 4, Seite 32).

Die Wahl der aufgeführten hämodynamischen und autonomen Parameter liegt in Erfahrungen vorangegangener Studien begründet (Lache 2006, Herrmann-Lingen et al. 2010). Mit diesen Werten sind alle vier Dimensionen sympathischer und kardiovaskulärer Aktivierung (alpha-adrenerg, betaadrenerg, rhythmische sowie nicht-rhythmische HRV-Analyse) abgedeckt, wie es von HerrmannLingen et al. (2010) empfohlen wurde.

In der detaillierten Analyse der Stressreaktivität sollen im Rahmen der vorliegenden Studie zunächst folgende fünf Parameter in die Hypothesentestung eingeschlossen werden:

- $\quad$ Cardiac Index $(\mathrm{CI}),\left[1 /\left(\min ^{*} \mathrm{~m}^{2}\right)\right]$ 
- totaler peripherer Widerstands-Index (TPRI), [dyne* $\mathrm{s}^{*} \mathrm{~m}^{2} / \mathrm{cm}^{5}$ ]

- Standardabweichung aller RR-Intervalle (SDNN), [ms]

- Niederfrequenzband der Herzratenvariabilität (LF-HRV), $\left[\mathrm{ms}^{2}\right]$

- Hochfrequenzband der Herzratenvariabilität (HF-HRV), [ms $\left.{ }^{2}\right]$.

Durch die Beschränkung auf diese fünf Parameter wird eine gute Übersichtlichkeit gewährleistet. Die übrigen Parameter standen für eine anschließende explorative Betrachtung zur Verfügung.
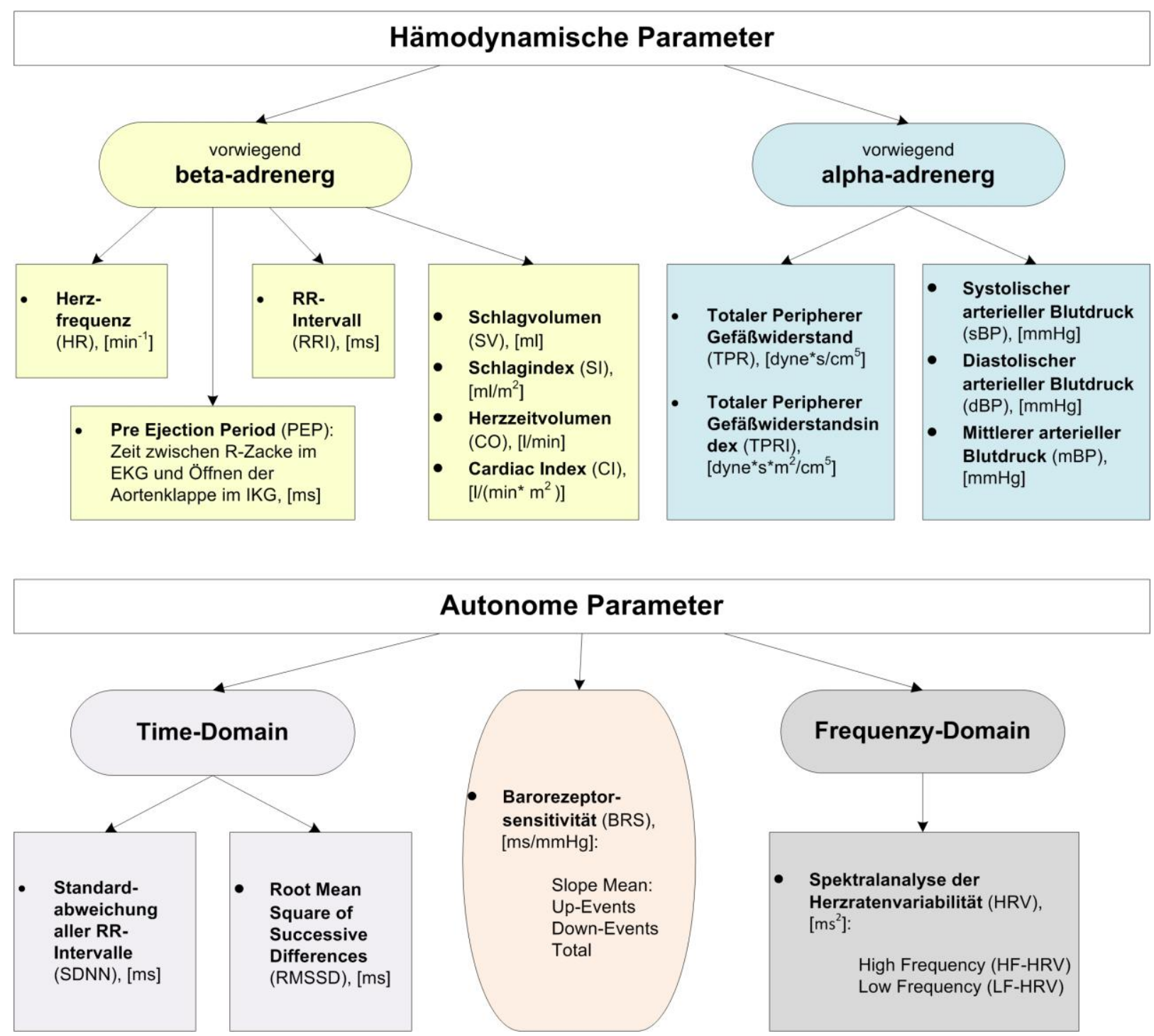

Abbildung 4: Hämodynamische und autonome Task-Force-Monitor-Parameter zur Beurteilung der Stressreaktion der (k)PTBS-Patientinnen

\subsubsection{Erholung nach Stressanforderung}

Bei der posttraumatischen Belastungsstörung wird eine verzögerte Rückbildungsfähigkeit physiologischer Parameter nach Stressanforderung diskutiert (Pole 2007, Sack et al. 2004, Keary et al. 2009). Um zu überprüfen, ob auch bei unserem emotionalen Stressor (Babyschreien) eine inadäquate 
Herabregulation der Stressparameter erfolgt, wurden neben dem Vergleich der Phasenmittelwerte zusätzliche Messzeitpunkte folgender Parameter analysiert:

- Cardiac Index (CI)

- $\quad$ systolischer Blutdruck (sBP)

- $\quad$ totaler peripherer Gefäßwiderstands-Index (TPRI)

- Root Mean Square of Successive Differences (RMSSD).

Anhand dieser vier Parameter sollen beispielhaft kurzfristige Änderungen der kardiovaskulären Erregung auf verschiedenen Ebenen beleuchtet werden. Aus dem Zeitbereich der HRV-Parameter wurde hier die RMSSD gewählt, da sie besonders gut für Kurzzeitanalysen geeignet ist. Die HRVParameter aus dem Frequenzbereich konnten aufgrund der kurzen Messdauer hier nicht ausgewertet werden. Es wurden für die ausgewählten Parameter jeweils 5 Schläge zu einem Mittelwert für die folgenden Messzeitpunkte zusammengefasst:

- Babyschreien: 4:30 min (30 Sekunden vor Ende des Babyschreiens)

- Ruhe 3: 0:30 min (nach 30 Sekunden Erholung)

- Ruhe 3: 1:00 min (nach 60 Sekunden Erholung)

- Ruhe 3: 1:30 min (nach 90 Sekunden Erholung).

\subsubsection{Anbringen der Messvorrichtung am Patienten}

Für die Untersuchung wurden der Patientin zunächst alle Elektroden angelegt. Zur Ableitung des bipolaren Zwei-Kanal-EKGs wurden vier an Klebeelektroden befestigte Clips entsprechend der Ableitungen I und II nach Einthoven am Oberkörper der Patientin angebracht. Für die Impedanzkardiographie wurden spezielle Bandelektroden im Bereich des Nackens und beidseits am Thorax auf Höhe des Xiphoids aufgeklebt und die entsprechenden Kontaktklemmen angeschlossen. Außerdem wurde die Neutralelektrode an das rechte Schienbein der Patientin angelegt. Nach Auswahl der passenden Fingermanschette wurde diese mit dem Task Force Vascular Unloading Monitor verbunden und über zwei benachbarte Finger (II und III) der Patientin geschoben und am rechten Unterarm der Patientin befestigt. Im Anschluss wurde die oszillometrische Oberarmmanschette der passenden Größe am kontralateralen Arm angelegt. Die Patientinnen mussten sich während der Untersuchung nicht vollständig entkleiden.

\subsubsection{Auswertung und Ausschluss von Task-Force-Monitor-Daten}

Für die vorliegende Arbeit wurde das TFM-Programm der Version 2.1 verwendet. Alle aufgenommenen Messwerte wurden an den angeschlossenen Laptop übertragen und dort mit Hilfe der TFM-Software dargestellt und gespeichert. Nach Einweisung und Instrumentierung der Patientin, Anlegen einer neuen Messdatei (Eingabemaske mit Abfrage von Patientinnen-Code, Geschlecht, Geburtsdatum, Größe und Gewicht) und Start der Messung erfolgten die Kalibrierungen automatisch zum Beginn der Messung und nach Manschettenwechsel, sowie bei Bedarf manuell. In verschiedenen 
Darstellungsmodi wurden diverse Kreislaufparameter und Langzeitverläufe während der Messung in Echtzeit dargestellt. Nach Abschluss der Messung wurden die aufgezeichneten Daten in einer *.fefDatei abgelegt. Um die erhobenen Daten weiter verarbeiten zu können, wurden die Daten in zwei verschiedene Microsoft Excel-Dateien (*.xls) sowie in eine *.pdf-Datei exportiert.

In einer anschließenden Plausibilitätskontrolle wurde die Qualität der erhobenen TFM-Daten überprüft. Als Ausschlusskritierien einzelner Phasenmittelwerte galten eine zu geringe Anzahl vorhandener Messwerte in einer Phase oder eine große Streuung der Messwerte, die zumeist auf fehlerhafte bzw. einen Ausfall von Messsignalen (z.B. Elektrodenablösung) oder auf höhergradige Herzrhythmusstörungen zurückzuführen waren. Die HRV-Parameter HF- und LF-HRV sowie RMSSD wiesen keine Normalverteilung auf und wurden deshalb zur weiteren Auswertung logarithmiert.

\subsection{Bildung der Patientinnen-Subgruppe „hoch Borderline“6}

Im Rahmen der vorliegenden Studie soll neben der Betrachtung des Gesamtkollektivs der PTBSPatientinnen auch eine spezifische Subgruppe mit einer stark ausgeprägten Borderline-Symptomatik anhand von psychometrischen Befunden identifiziert werden. Es gilt zu überprüfen, ob diese Subgruppe unterschiedliche Reagibilitätsmuster während der Stressbelastungsuntersuchung im Vergleich zum Rest des Ausgangskollektivs aufweist.

Die Patientinnen-Subgruppe „hoch Borderline“ wurde anhand im Rahmen der Borderline-Diagnostik weit verbreiteter und etablierter Selbstbeurteilungsinstrumente, Borderline-Persönlichkeits-Inventar (BPI; Leichsenring 1997) sowie Borderline-Symptom-Liste (BSL, Bohus et al. 2001), gebildet (Abbildung 5, Seite 35).

Ein auf 20 Items des BPI basierender Cut-Off-Wert (Cut-20) dient im klinischen Alltag der Abgrenzung von Borderline-Patienten von neurotischen bzw. schizophrenen Patienten. Bei einem Kriterium Cut-20 $\geq 10$ kann eine BPS als sehr wahrscheinlich angenommen werden, während ein Cut$20 \leq 10$ als ein mögliches etabliertes Ausschlusskriterium einer BPS-Diagnose gilt. Für die Bildung der Patientinnen-Untergruppe „hoch Borderline“ wurde deshalb als grundlegendes Kriterium ein Cut$20 \geq 10$ des BPI definiert.

Die BSL dient der Quantifizierung subjektiv erlebter intrapsychischer Belastung und Beeinträchtigung von Borderline-Patienten und gibt daher Auskunft über die Schweregradausprägung der BorderlineSymptomatik. Die BPS zeichnet sich durch ein tiefgreifendes Muster von Instabilität der Affektregulation, Impulskontrolle, in zwischenmenschlichen Beziehungen und im Selbstbild aus. Unter der Annahme, dass insbesondere eine gestörte Affektregulation mit impulsiven und autoaggressiven Tendenzen Einfluss auf die Stressphysiologie der (k)PTBS-Patientinnen haben könnte, wurden als zusätzliche Kriterien für die Subgruppen-Bildung die Subskalen Affektregulation und Autoaggression der BSL gewählt. Da durch die Subgruppe „hoch Borderline“ eine besonders stark ausgeprägte Borderline-Symptomatik abgebildet werden sollte, wurde für die Zugehörigkeit zur 
Subgruppe jeweils ein Wert größer als der Median des Gesamtkollektivs festgelegt. Gemäß den Medianen ergaben sich für die BSL-Subskala Affektregulation ein Wert >25 und für die BSLSubskala Autoaggression ein Wert $>17,5$.

Durch diese komplexen, drei Bedingungen erfüllenden Definitionskriterien (BPI: Cut-20 $\geq 10$, BSL: Subskala Affektregulation >25 und Subskala Autoaggression >17,5; Abbildung 5, Seite 35) sollte eine im Vergleich zum Rest des Ausgangskollektivs deutlich stärkere Borderline-Symptomausprägung in der Subgruppe „hoch Borderline“ gewährleistet werden, um der Forschungslücke des Einflusses einer ausgeprägten Borderline-Symptomatik auf die Stressphysiologie von (k)PTBS-Patientinnen näher auf den Grund gehen zu können.

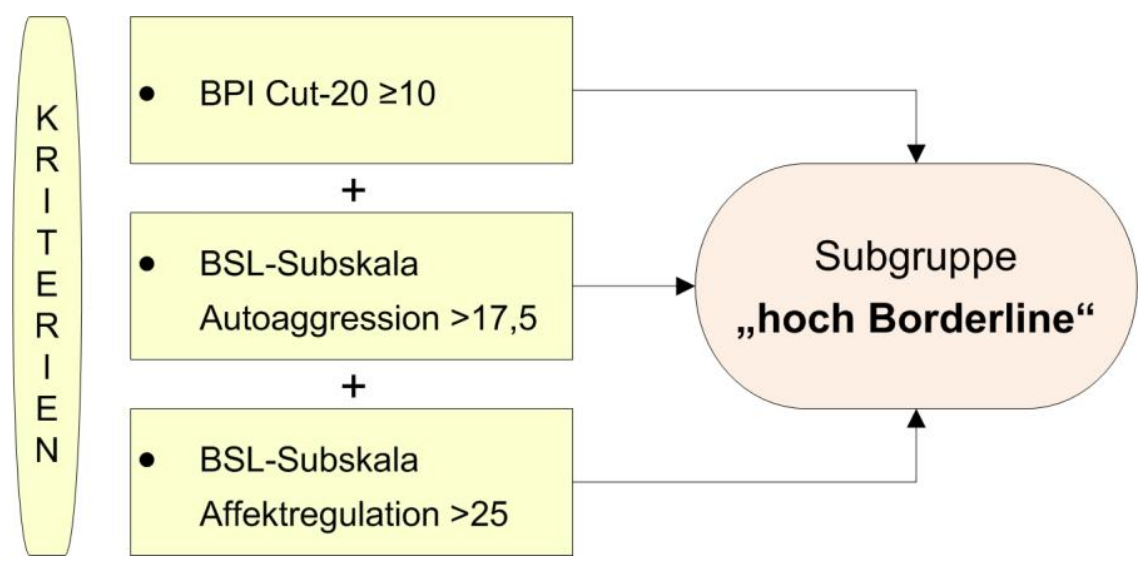

Abbildung 5: Kriterien für die Zugehörigkeit zur Subgruppe „hoch Borderline“، „Hoch Borderline“ wird angenommen, wenn alle drei genannten Kriterien erfüllt sind.

Als Ausgangskollektiv standen für die Bildung der Subgruppe die Daten von 46 Patientinnen zur Verfügung. Sechs Patientinnen konnten nicht berücksichtigt werden, da sie das Fragebogen-Set der Station 9 des Asklepios Fachklinikums nicht ausgefüllt hatten. Zwei weitere Patientinnen wurden aufgrund von höhergradigen Herzrhythmusstörungen von der Betrachtung der physiologischen Parameter ausgeschlossen.

\subsection{Datenverarbeitung und statistische Auswertung}

Die erhobenen Daten wurden auf einem Personalcomputer der Klinik für Psychosomatische Medizin und Psychotherapie der UMG in pseudonymisierter Form als MS-Excel-Dateien gespeichert. Die statistische Auswertung der Untersuchungsergebnisse erfolgte nach Plausibilitätskontrolle unter Zuhilfenahme der Statistik-Software SAS (Version 9.2) und SPSS (Version 20). Die Graphiken für die TFM-Messung wurden mit Statistica (Version 10) erstellt. Die Daten der diagnostischen Interviews, der Fragebögen sowie die klinischen Daten wurden manuell kodiert und unmittelbar als SPSS-Datei gespeichert und mit diesem Programm ausgewertet. 


\subsubsection{Allgemeines}

Die Beschreibung des Patientinnenkollektivs erfolgte deskriptiv mithilfe der Statistik-Software SPSS für MS-Windows, Version 20. Bei der Darstellung von Häufigkeitsverteilungen für soziodemographische und klinische Daten wurden als Prozentwerte grundsätzlich gültige Prozente angegeben. Für die psychometrischen Fragebogen-Skalen wurden zur deskriptiven Statistik nach Ausschluss unvollständiger Datensätze Mittelwerte und Standardabweichung sowie minimaler bzw. maximaler Wert ausgewählt. Die Patientinnen-Subgruppe wurde mit dem Rest des Ausgangskollektivs, welcher die Kriterien der Subgruppenzugehörigkeit nicht erfüllte, bezüglich soziodemographischer und psychometrischer Daten verglichen. Dazu dienten für normalverteilte Daten t-Tests für unverbundene Stichproben, nichtparametrische Daten wurden mittels U-Test nach Mann-Whitney bzw. für kategoriale Variablen mittels $\mathrm{Chi}^{2}$-Test verglichen.

Als Signifikanzkriterium wurde für alle Analysen der Fehler 1. Art von alpha=5\% definiert, wobei der p-Wert jeweils in den drei Klassen $\mathrm{p}<0,05,<0,01$ und $<0,001$ angegeben wird.

\subsubsection{Analyse der Task-Force-Monitor-Daten}

Für die Auswertung der TFM-Daten erfolgte in Zusammenarbeit mit dem Institut für medizinische Statistik eine einfaktorielle Varianzanalyse für Messwiederholungen (repeated measures ANOVA) mit der Statistik-Software SAS, Version 9.2. Mit diesem globalen Test konnte überprüft werden, inwiefern sich die einzelnen physiologischen TFM-Parameter (Phasenmittelwerte pro Patientin) während der Phasen der Stressbelastungsuntersuchung unterschiedlich verhielten. Anschließende Paarvergleiche wurden mittels verbundenem/ unverbundenem t-Test durchgeführt. Eine Adjustierung nach Bonferroni wurde angewandt. Dabei wurden folgende, als wesentlich erachtete sechs Paarvergleiche überprüft:

- Ruhe 1 vs. Rechentest

- Ruhe 1 vs. Ruhe 2

- Ruhe 1 vs. Babyschreien

- Ruhe 1 vs. Ruhe 3

- Rechentest vs. Babyschreien

- Ruhe 1 vs. Entspannungsmusik.

Ergab sich hierbei ein signifikanter Unterschied im Paarvergleich, so wurde die Differenz der Phasenmittelwerte \pm Standardfehler für das entsprechende Messphasen-Paar angegeben. Auf gleiche Weise erfolgte die Auswertung der Erholungsphase (Recovery) nach Babyschreien für die im Methodenteil (Kapitel 3.7.6, Seite 32) beschriebenen festgelegten vier Messzeitpunkte sowie ausgewählten vier TFM-Parameter.

Für einen direkten Vergleich der Effekte beider Stressoren (Rechentest und Babyschreien) wurden jeweils die Phasenmittelwert-Differenzen der Ausgangssituation (Ruhe 1) vs. Rechnen bzw. Babyschreien berechnet und mittels gepaartem t-Test vergleichen. Positive Phasenmittelwert- 
Differenzen werden in der vorliegenden Arbeit als Anstieg bzw. Zunahme des physiologischen Parameters bezeichnet und analog dazu negative als Abfall bzw. Abnahme.

Für die Beurteilung der Stressreagibilitätsmuster der Patientinnen-Subgruppe „hoch Borderline“ wurde eine zweifaktorielle ANOVA für Messwiederholungen gewählt, da die Subgruppe dem Rest des Ausgangskollektivs zum Vergleich gegenübergestellt und eine mögliche Interaktion über die Phasen aufgedeckt werden sollte. Des Weiteren erfolgte ein Vergleich der PhasenmittelwertDifferenzen des Paarvergleichs Rechnen vs. Babyschreien zwischen den Gruppen mittels t-Test.

\subsubsection{Auswertung der subjektiven Aktivierung (Analogskala „Stressempfinden“ und emotionale bzw. vegetative Reaktionen)}

Die Angaben der Patientinnen zum Stresserleben auf der Subjektivskala wurden neben einer deskriptiven Statistik (Mittelwert \pm Standardabweichung, Median, Minimum, Maximum) auch einer globalen ANOVA- für Messwiederholungen mit anschließendem Paarvergleich unterzogen. Somit konnte das Stressempfinden während der Phasen der Stressbelastungsuntersuchung umfangreich charakterisiert werden. Auch die emotionalen bzw. vegetativen Reaktionen während der Messung wurden mittels einfaktorieller ANOVA analysiert und mittels Häufigkeitsverteilung für die einzelnen Messphasen dargestellt.

Die Ergebnisse der Patientinnen-Subgruppe „hoch Borderline“ wurden mittels U-Test nach MannWhitney bzw. Chi ${ }^{2}$-Test nach Pearson mit der Vergleichsgruppe verglichen.

Zudem erfolgte eine Korrelationsanalyse nach Kendall-Tau-b, um einen Zusammenhang zwischen der Anzahl beobachteter vegetativer Reaktionen und den physiologischen Parametern während des Babyschreiens zu untersuchen (für das Gesamtkollektiv und als Subgruppenvergleich). Eine Korrelation von Skalen mit wenigen (ordinalen) Abstufungen und metrischen Daten wird mit dem Konzept der Rangkorrelation gelöst. Hier steht Kendalls Tau oder Spearmans Rho zur Verfügung. Kendalls Tau ist sehr robust und wurde deshalb gewählt. 


\section{Ergebnisse}

\subsection{Beschreibung des Patientinnenkollektivs}

\subsubsection{Vollständigkeit der Daten}

Das Patientinnenkollektiv der Studie umfasste insgesamt 54 Patientinnen mit Verdacht auf eine (komplexe) posttraumatische Belastungsstörung, welche die oben genannten Einschlusskriterien erfüllten und schriftlich eingewilligt hatten.

Von der Gesamtkohorte mussten die TFM-Messungen bzw. einzelne Messwerte oder Phasenmittelwerte von vier Patientinnen aus unterschiedlichen Gründen von der weiteren Betrachtung ausgeschlossen werden. Zwei Patientinnen wiesen höhergradige Überleitungsstörungen im EKG auf, die eine sinnvolle Bestimmung der HRV und anderer Parameter verhinderten. Bei einer weiteren Teilnehmerin konnten die Phasenmittelwerte des Cardiac Index (CI) sowie des Schlagindex (SI) aufgrund $\mathrm{zu}$ vieler Artefakte des Impedanzsignals (vermutlich durch Elektrodenablösung) nicht ausgewertet werden. Eine Patientin brach die Messung vorzeitig ab. Insgesamt standen somit 50 vollständige Datensätze zur weiteren Auswertung der physiologischen Stressparameter zur Verfügung. Sechs Patientinnen lehnten das Ausfüllen des Fragebogensets im Rahmen der Diagnostik auf der Station 9 des AFG ab. Von den verbleibenden 48 Datensätzen sind fünf unvollständig, da einzelne Fragebögen von den Patientinnen nicht ausgefüllt wurden. Von allen 54 Patientinnen lagen die Ergebnisse der diagnostischen Interviews mit erfasster DSM-IV bzw. IDC-10 Diagnose vor (APA 2003, WHO 2006). Abbildung 6 (Seite 38) zeigt die Vollständigkeit der Datensätze für die unterschiedlichen Untersuchungsabschnitte.

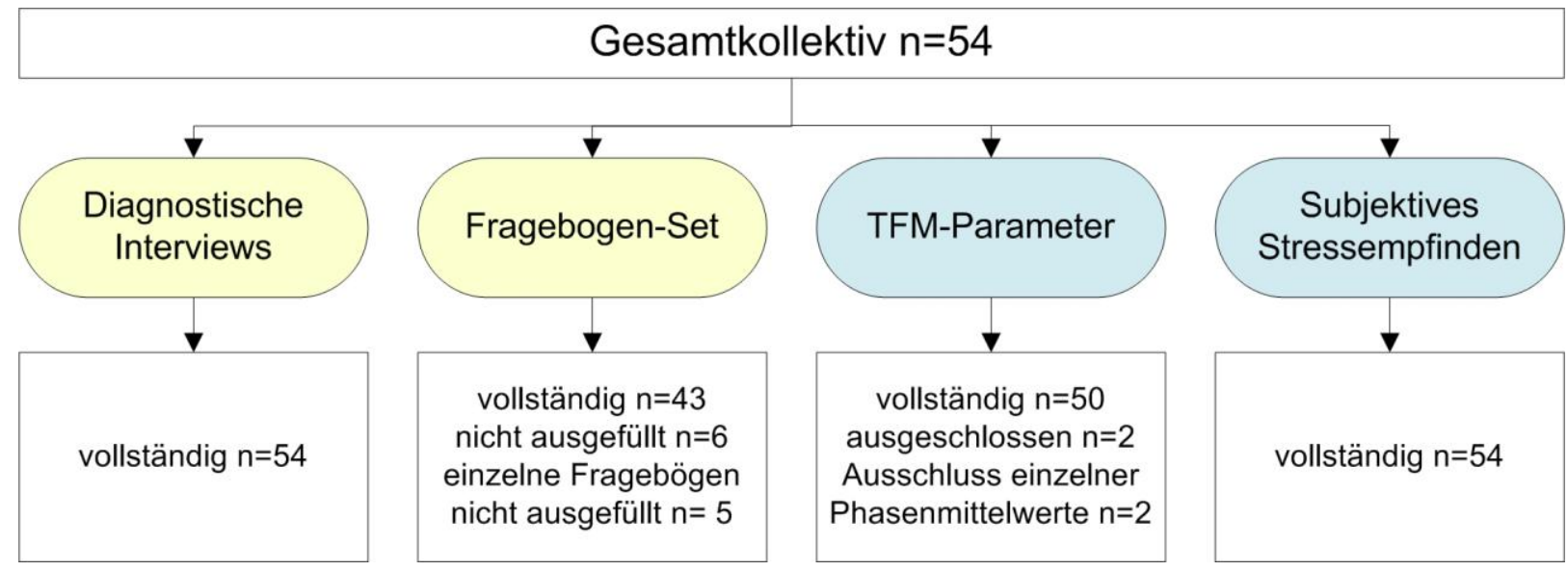

Abbildung 6: Vorhandene Datensätze [n] für die Teiluntersuchungen der Studie (Diagnostische Interviews, Fragebögen, TFM-Messung, Analogskala zum subjektiven Stressempfinden) 


\subsubsection{Soziodemographische Daten}

An der Studie nahmen Patientinnen im Alter von 20 bis 56 Jahren teil mit einem Altersdurchschnitt von 41,4 Jahren (SD=8,69). Das Balkendiagramm der Abbildung 7 (Seite 39) zeigt die Altersverteilung des Patientinnenkollektivs zum Zeitpunkt der Messung.

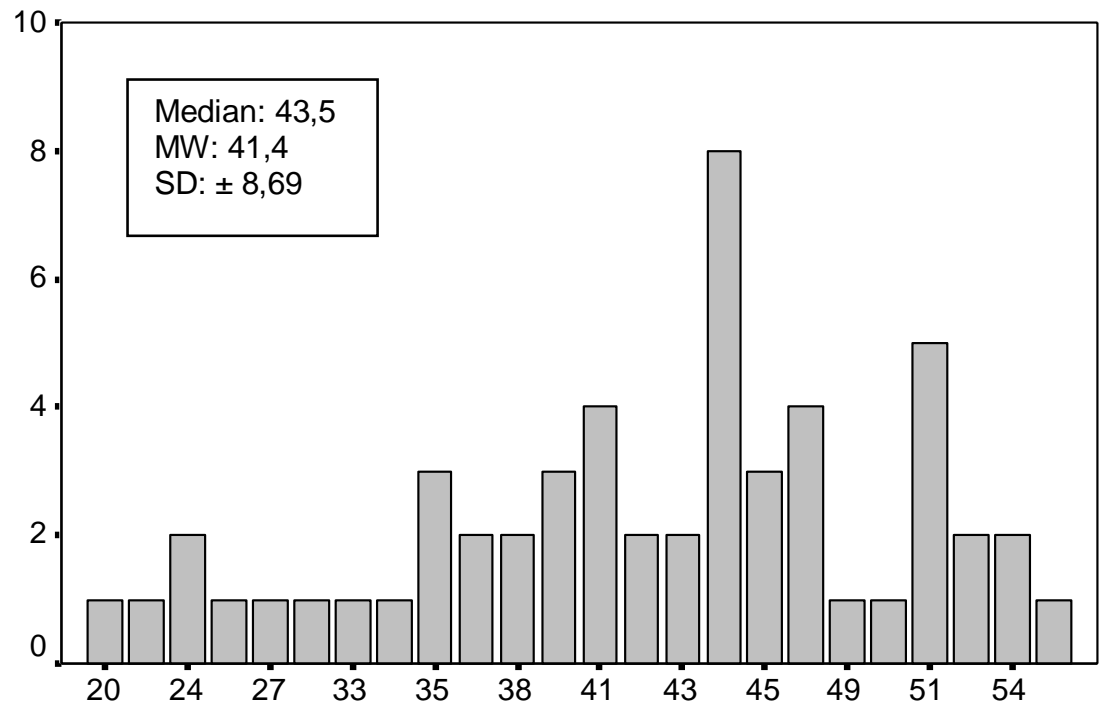

Alter in Jahren

\section{Abbildung 7: Altersverteilung des Gesamtkollektivs (n=54)}

Soziodemographische Fakten zum Familien- und Bildungsstand sind in Tabelle 3 (Seite 40) zusammenfassend dargestellt.

Es wurden folgende Angaben zum Familienstand erhoben: etwa ein Drittel $(35,2 \%)$ der Patientinnen war verheiratet, jeweils 31,5\% gaben an ledig bzw. geschieden zu sein, 1,9\% waren verwitwet. 40,7\% der Patientinnen lebten mit ihrem Partner zusammen, $13 \%$ befanden sich zwar in einer festen Partnerschaft, lebten jedoch nicht mit ihrem Partner zusammen. Die Mehrzahl der befragten Frauen hatte Kinder $(68,5 \%)$.

Hinsichtlich des Bildungsstandes ist folgendes festzuhalten: 14,8\% der Patientinnen beendeten die Hauptschule, etwa ein Drittel hatte einen Realschulabschluss (33,3\%) und 27,8\% absolvierten das Abitur. Betrachtet man den beruflichen Werdegang, so befanden sich 5,6\% der Patientinnen zum Untersuchungszeitpunkt noch in der Ausbildung, 9,3\% hatten keinen berufsqualifizierenden Abschluss. Die Hälfte der Patientinnen hatte einen Lehrberuf erlernt, 14,8\% hatten eine Fach-, Meister bzw. Technikerschule oder Berufs- bzw. Fachakademie besucht, 11,7\% erworben einen Fachhochschul- und 7,1\% einen Hochschulabschluss. Die Mehrzahl der Patientinnen war jedoch zum Zeitpunkt der Messung nicht berufstätig (64,8\%), nur 20,4\% arbeiteten Vollzeit (>35h/Woche). 
Tabelle 3: Soziodemographische Daten des Gesamtkollektivs zum Zeitpunkt der Messung

\begin{tabular}{|c|c|c|}
\hline \multicolumn{2}{|c|}{$\begin{array}{l}\text { Soziodemographische Daten } \\
\text { Gesamtkollektiv, } n=54\end{array}$} & $\begin{array}{c}\text { Häufigkeiten } \\
\text { (Prozente) }\end{array}$ \\
\hline \multirow{4}{*}{ Familienstand } & ledig & $17(31,5 \%)$ \\
\hline & verheiratet & $19(35,2 \%)$ \\
\hline & geschieden & $17(31,5 \%)$ \\
\hline & verwitwet & $1(1,9 \%)$ \\
\hline \multirow{3}{*}{$\begin{array}{c}\text { Feste Partnerschaft/ } \\
\text { Zusammenleben mit Partner }\end{array}$} & nein & $25(46,3 \%)$ \\
\hline & ja/nicht zusammenlebend & $7(13,0 \%)$ \\
\hline & ja/zusammenlebend & $22(40,75)$ \\
\hline \multirow{6}{*}{ Eigene Kinder } & keine Kinder & $17(31,5 \%)$ \\
\hline & $1 \mathrm{Kind}$ & $15(27,8 \%)$ \\
\hline & 2 Kinder & $13(24,1 \%)$ \\
\hline & 3 Kinder & $5(9,3 \%)$ \\
\hline & 4 Kinder & $3(5,6 \%)$ \\
\hline & 5 Kinder & $1(1,9 \%)$ \\
\hline \multirow{6}{*}{ Schulabschluss } & Hauptschule & $8(14,8 \%)$ \\
\hline & Realschule & $18(33,3 \%)$ \\
\hline & Polytechnische Oberschule & $4(7,4 \%)$ \\
\hline & Fachhochschule & $8(14,8 \%)$ \\
\hline & Gymnasium & $15(27,8 \%)$ \\
\hline & anderer Schulabschluss & $1(1,9 \%)$ \\
\hline \multirow{7}{*}{ Ausbildungsart } & keine Ausbildung/Abschluss & $5(9,3 \%)$ \\
\hline & beruflich-betriebliche Ausbildung & $14(25,9 \%)$ \\
\hline & schulische Ausbildung & $13(24,1 \%)$ \\
\hline & $\begin{array}{l}\text { Fach-/Meister-/Technikerschule/ } \\
\text { Berufs-/Fachakademie }\end{array}$ & $8(14,8 \%)$ \\
\hline & Fachhochschule & $4(7,4 \%)$ \\
\hline & Hochschule & $6(11,1 \%)$ \\
\hline & anderer Berufsabschluss & $4(7,4 \%)$ \\
\hline \multirow{2}{*}{ Ausbildung beendet? } & ja & $51(94,4 \%)$ \\
\hline & Nein & $3(5,6 \%)$ \\
\hline \multirow{4}{*}{ Berufstätigkeit } & nicht erwerbstätig & $35(64,8 \%)$ \\
\hline & $<15 \mathrm{~h} /$ Woche & $4(7,4 \%)$ \\
\hline & 15-35h/Woche & $4(7,4 \%)$ \\
\hline & >35h/Woche (Vollzeit) & $11(20,4 \%)$ \\
\hline
\end{tabular}

Betrachtet man das Risikoprofil der Patientinnen ergibt sich folgendes Bild (Tabelle 4, Seite 41): 35,2\% haben einen erhöhten Body Mass Index (BMI 25-30), welcher im Bereich einer Präadipositas einzuordnen ist, 29,6\% sind adipös (BMI >35). Der Mittelwert für den BMI lag bei 27,72 (SD=6,45). 42,6\% der Patientinnen gehen keiner regelmäßigen sportlichen Betätigung nach, der Großteil treibt weniger als zwei Stunden Sport pro Woche (38,9\%), im Durchschnitt 1,27 Stunden pro Woche $(\mathrm{SD}=1,93)$. Die subjektiven Angaben zum Alkoholkonsum ergaben, dass die Mehrzahl der Patientinnen keinen Alkohol trinkt (68,6\%), der maximal angegebene Wert lag bei 30 drinks per week, der Mittelwert bei 2,61 drinks per week (SD=6,33). Über die Hälfte (57,4\%) der Patientinnen raucht, im Durchschnitt weisen sie 13,04 packyears auf ( $\mathrm{SD}=16,71)$. 
Tabelle 4: Risikoprofil des Gesamtkollektivs zum Zeitpunkt der Messung

\begin{tabular}{|c|c|c|c|c|c|c|}
\hline \multicolumn{2}{|c|}{$\begin{array}{l}\text { Risikoprofil } \\
\text { Gesamtkollektiv, } n=54\end{array}$} & $\begin{array}{c}\begin{array}{c}\text { Häufigkeiten } \\
\text { (Prozente) }\end{array}\end{array}$ & Mittelwert & $\begin{array}{l}\text { Standard- } \\
\text { abweichung }\end{array}$ & Minimum & Maximum \\
\hline \multirow{4}{*}{$\begin{array}{c}\text { Body Mass } \\
\text { Index BMI } \\
(\mathrm{kg} / \mathrm{m} 2)\end{array}$} & $18,5-25$ & $19(35,2 \%)$ & \multirow{4}{*}{27,72} & \multirow{4}{*}{6,45} & \multirow{4}{*}{18,93} & \multirow{4}{*}{44,92} \\
\hline & $25-30$ & $19(35,2 \%)$ & & & & \\
\hline & $30-35$ & $8(14,8 \%)$ & & & & \\
\hline & $>35$ & $8(14,8 \%)$ & & & & \\
\hline \multirow{6}{*}{$\begin{array}{l}\text { Sportliche } \\
\text { Betätigung } \\
\text { (h/Woche) }\end{array}$} & kein Sport & $23(42.6 \%)$ & \multirow{6}{*}{1,27} & \multirow{6}{*}{1,93} & \multirow{6}{*}{0} & \multirow{6}{*}{$>10$} \\
\hline & $<0,5$ & $4(7,4 \%)$ & & & & \\
\hline & $0,5-2$ & $17(31,5 \%)$ & & & & \\
\hline & $2-5$ & $8(14,8 \%)$ & & & & \\
\hline & $5-10$ & $1(1,9 \%)$ & & & & \\
\hline & $>10$ & $1(1,9 \%)$ & & & & \\
\hline \multirow{4}{*}{$\begin{array}{c}\text { Alkohol- } \\
\text { konsum } \\
\text { (drinks per } \\
\text { week) }\end{array}$} & kein Alkohol & $37(68,6 \%)$ & \multirow{4}{*}{2,61} & \multirow{4}{*}{6,33} & \multirow{4}{*}{0} & \multirow{4}{*}{30} \\
\hline & $0,5-5 \mathrm{dpw}$ & $8(14,8 \%)$ & & & & \\
\hline & 5-10 dpw & $6(11,1 \%)$ & & & & \\
\hline & $>10 \mathrm{dpw}$ & $3(5,6 \%)$ & & & & \\
\hline \multirow{5}{*}{$\begin{array}{c}\text { Tabak- } \\
\text { abusus } \\
\text { (packyears) }\end{array}$} & Nichtraucherin & $23(42,6 \%)$ & \multirow{5}{*}{13,04} & \multirow{5}{*}{16,71} & \multirow{5}{*}{0} & \multirow{5}{*}{70} \\
\hline & $0,5-5$ py & $4(7,4 \%)$ & & & & \\
\hline & 5-10 py & $6(11,1 \%)$ & & & & \\
\hline & $10-20$ py & $8(14,8 \%)$ & & & & \\
\hline & $>20$ py & $13(24,1 \%)$ & & & & \\
\hline
\end{tabular}

\subsubsection{Klinische Daten}

\subsubsection{Traumafolgestörungen}

Das Gesamtkollektiv setzt sich bezüglich der traumaassoziierten Störungsbilder folgendermaßen zusammen (Abbildung 8, Seite 42): der Verdacht einer PTBS hat sich bei allen 54 Patientinnen bestätigt. Über $3 / 4$ der Patientinnen (79,6\%) erhielten die Diagnose chronifizierte PTBS nach Trauma Typ II (seltener nach Trauma Typ I), welche nach der ICD-10-Klassifikation den Schlüssel F43.1 trägt (WHO 2006). Eine komplexe PTBS (kPTBS) gemäß DESNOS-Kriterien (Disorder of Extreme Stress Not Otherwise Specified, DSM-IV, APA 2003) lag bei 11 Patientinnen (20,4\%) vor. 34 Patientinnen (63\%) litten zusätzlich an einer dissoziativen Symptomatik in Form einer dissoziativen Amnesie, Fugue, nicht näher bezeichneter dissoziativen Störung bzw. Identitätsstörung oder einer Depersonalisation bzw. Derealisation. Im Rahmen der zusätzlich zur PTBS ausgeprägten Persönlichkeitsstörungen (PS) überwog die emotional instabile Persönlichkeitsstörung vom Borderline-Phänotyp mit 40,7\%. Fünf Patientinnen (9,3\%) hatten eine andere Persönlichkeitsstörung (ängstlich-vermeidend, abhängig, histrionisch). 13 Patientinnen $(24,1 \%)$ wiesen zusätzlich zur PTBS bzw. kPTBS sowohl eine dissoziative Störung als auch eine Borderline-Persönlichkeitsstörung auf. 


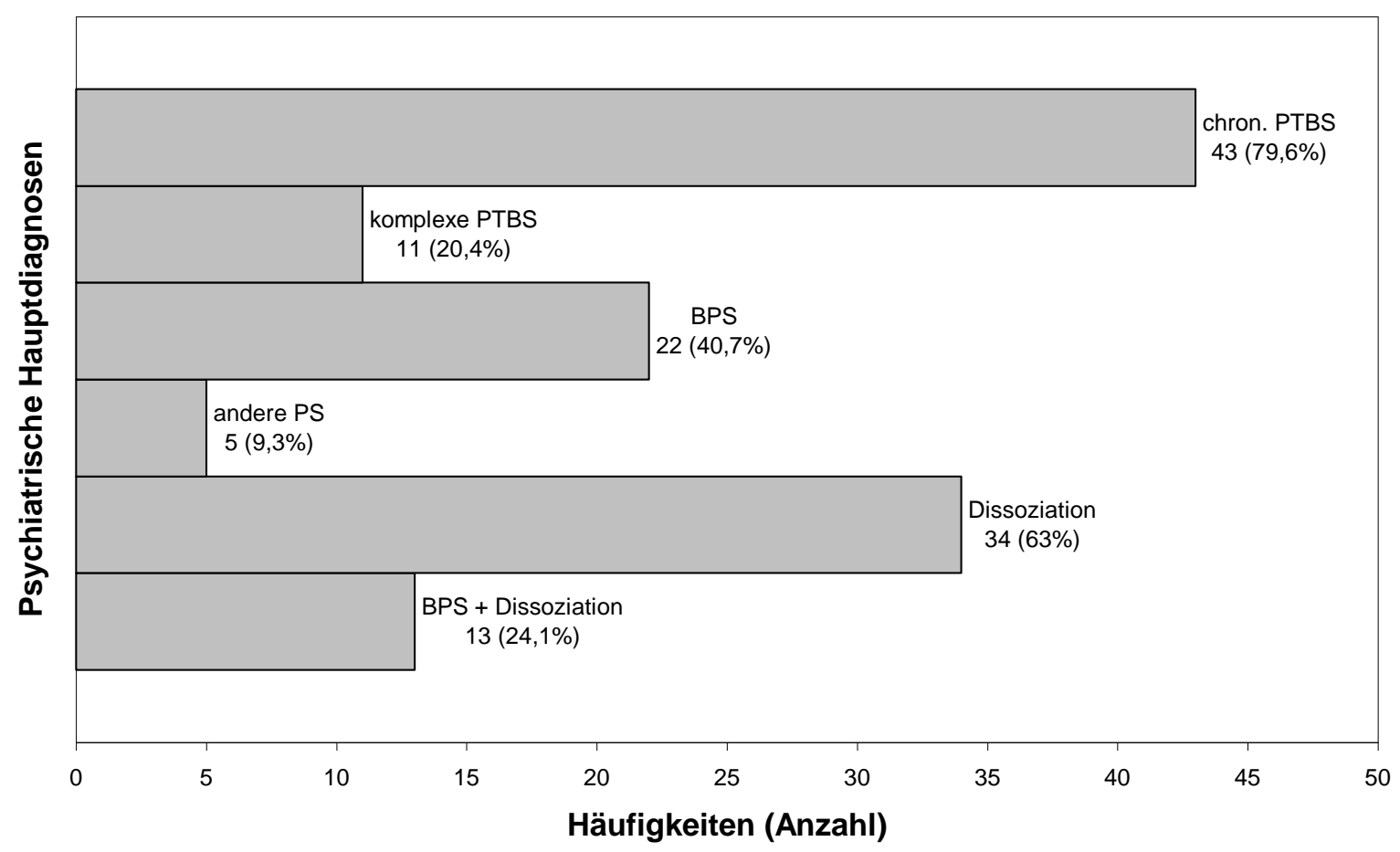

Abbildung 8: Häufigkeitsverteilung (Anzahl und Prozente) der traumaassoziierten Störungsbilder im Gesamtkollektiv $(\mathbf{n}=54)$

\subsubsection{Begleitdiagnosen}

Bezüglich weiterer Komorbiditäten ergibt sich folgendes Bild (Tabelle 5, Seite 43): der Großteil der Patientinnen (96,3\%) hatte als psychiatrische Nebendiagnose eine rezidivierende depressive Störung. Des Weiteren fanden sich bei jeweils etwa einem Drittel der Patientinnen $(31,5 \%)$ eine somatoforme Störung oder eine Angst- bzw. Panikstörung. 13 Patientinnen $(24,1 \%)$ litten unter einer Essstörung (Bulimia nervosa oder Anorexie). 40,7\% der Patientinnen zeigten selbstverletzendes Verhalten (SVV). Weitere häufige Begleiterkrankungen waren chronische Rückenschmerzen bzw. Bandscheibenvorfälle (33,3\%), Erkrankungen der Schilddrüse, v.a. Hypothyreose (25,9\%) sowie eine arterielle Hypertonie mit 18,5\% gefolgt von Atemwegserkrankungen, v.a. bronchiales Asthma (16,7\%), und Erkrankungen des Gastrointestinaltraktes, v.a. nervöse Magenbeschwerden und Reizdarmsyndrom (14,8\%). Des Weiteren waren Migräne sowie dermatologische Erkrankungen mit jeweils 9,3\% im Gesamtkollektiv zu finden. Herzrhythmusstörungen, in erster Linie anamnestisch erhoben, waren bei vier Patientinnen (7,4\%) zugegen, jedoch waren diese nur bei zwei Patientinnen höhergradig und führten daher zum Ausschluss aus der Auswertung der TFM-Messung. Selten vertretene Begleiterkrankungen waren Gefäßerkrankungen, Diabetes mellitus, Lebererkrankungen, v.a. Hepatitiden, Tinnitus, Tumorerkrankungen und Erkrankungen aus dem rheumatischen Formenkreis. 
Tabelle 5: Verteilung der Begleitdiagnosen im Gesamtkollektiv

\begin{tabular}{|l|c|}
\hline $\begin{array}{l}\text { Begleitdiagnosen } \\
\text { Gesamtkollektiv n= 54 }\end{array}$ & $\begin{array}{c}\text { Häufigkeiten } \\
\text { (Prozente) }\end{array}$ \\
\hline depressive Störung & $52(96,3 \%)$ \\
\hline somatoforme Störung & $17(31,5 \%)$ \\
\hline Angststörung & $17(31,5 \%)$ \\
\hline Essstörung & $13(24,1 \%)$ \\
\hline selbstverletzendes Verhalten (SVV) & $22(40,7 \%)$ \\
\hline $\begin{array}{l}\text { chronische Rückenschmerzen } \\
\text { /Bandscheiben-Probleme }\end{array}$ & $18(33,3 \%)$ \\
\hline Erkrankungen der Schilddrüse & $14(25,9 \%)$ \\
\hline arterielle Hypertonie & $10(18,5 \%)$ \\
\hline Herzrhythmusstörungen & $4(7,4 \%)$ \\
\hline Atemwegserkrankungen & $9(16,7 \%)$ \\
\hline Migräne & $5(9,3 \%)$ \\
\hline Erkrankungen des Gastrointestinaltraktes & $8(14,8 \%)$ \\
\hline Diabetes mellitus Typ 2 & $3(5,6 \%)$ \\
\hline Erkrankungen der Leber & $3(5,6 \%)$ \\
\hline dermatologische Erkrankungen & $5(9,3 \%)$ \\
\hline rheumatische Erkrankungen & $1(1,9 \%)$ \\
\hline Gefäßerkrankungen & $4(7,4 \%)$ \\
\hline Tumorerkrankungen & $2(3,7 \%)$ \\
\hline Tinnitus & $2(3,7 \%)$ \\
\hline & \\
\hline & 140 \\
\hline
\end{tabular}

\subsubsection{Suchtverhalten}

Das Suchtverhalten der Patientinnen lässt sich folgendermaßen beschreiben (Abbildung 9, Seite 43):

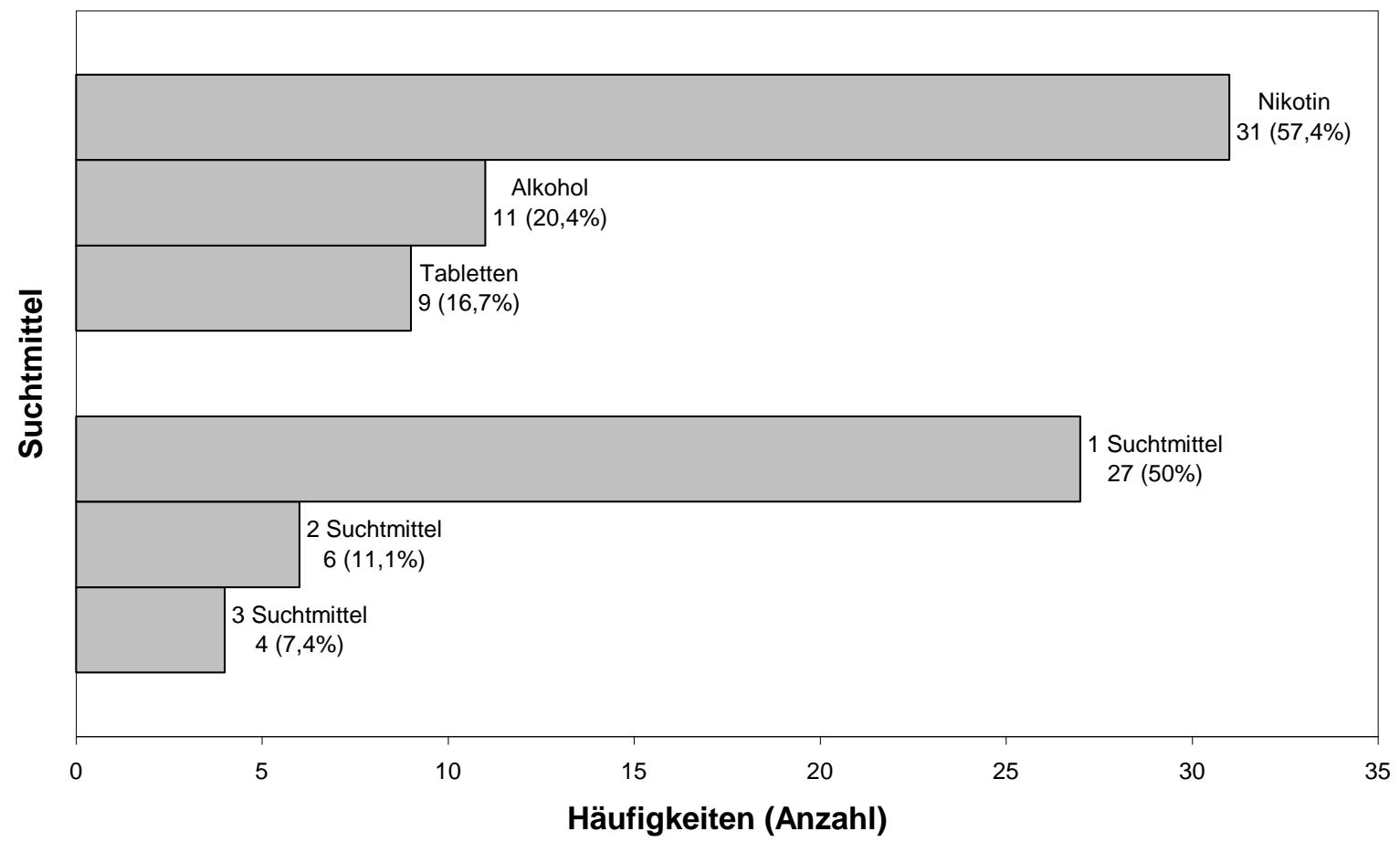

Abbildung 9: Suchtverhalten der Patientinnen ( $n=54)$ 
die Hälfte der Patientinnen nahm ein Suchtmittel zu sich, wobei Tabakkonsum überwiegt. 11 Patientinnen $(20,4 \%)$ hatten einen Alkoholabusus in der Vorgeschichte. Eine Tablettenabhängigkeit (v.a. Benzodiazepine oder Schmerzmittel) bestand bei neun Patientinnen (16,7\%). Der Anteil an Patientinnen, die sowohl rauchten, einen Alkoholabusus vor dem Stationsaufenthalt aufwiesen als auch tablettenabhängig waren, betrug $7,4 \%$.

\subsubsection{Phase des stationären Aufenthalts}

Der größere Anteil der Patientinnen (70,4\%) befand sich zum Zeitpunkt der Messung in der ein- bzw. zweiwöchigen Diagnostik- und Stabilisierungsphase auf der Station 9 des AFG. 16 Patientinnen (29,6\%) kamen zum ersten Therapieintervall mit dem Ziel der Traumasynthese.

\subsubsection{Traumaursache/Art der Traumatisierung}

Anhand eines Aktenstudiums wurde eine Übersicht über die Traumaursache bzw. Art der Traumatisierung erstellt. Ein Großteil der Patientinnen (48 von $54=88,9 \%$ ) hatte im Kindesalter eine Traumatisierung erfahren. Die Prävalenzzahlen lagen für sexuelle Gewalterfahrungen bei $74,0 \%$ und für physische Misshandlungen bei 63,0\%. Für die Mehrzahl der Patientinnen (51,85\%) sind Mehrfachtraumatisierungen dokumentiert. Es überwiegen eindeutig Typ-II-Traumata (länger anhaltend und wiederholt) mit 59,2\% im Vergleich zu Typ-I-Traumata (kurz und einmalig) mit 5,6\%. Bei 19 Patientinnen $(35,2 \%)$ hatten traumatisierende Erfahrungen vom Typ I und II stattgefunden.

\subsubsection{Medikamente}

Zur medikamentösen Behandlung der Patientinnen (Tabelle 6, Seite 45) wurden aus der Gruppe der Psychopharmaka am häufigsten Antidepressiva eingesetzt (72,2\%), wobei neben SSRI (29,6\%) und trizyklischen Antidepressiva (24,1\%) auch mehrheitlich andere Antidepressiva (38,9\%) z.B. aus der Gruppe der SNRI, tetrazyklische Antidepressiva oder Lithium gegeben wurden. 23 Patientinnen waren auf Neuroleptika eingestellt und über ein Drittel der Patientinnen (37,0\%) nahmen Tranquilizer bzw. Sedativa, wobei hier Benzodiazepine (33,3\%) als Bedarfsmedikation überwogen. Acht Patientinnen (14,8\%) nahmen regelmäßig Analgetika ein. Aus der Gruppe der Antiarrhythmika bzw. Antihypertensiva, die von etwa einem Viertel der Patientinnen eingenommen wurden, wurden mit $14,8 \%$ am häufigsten Betablocker eingesetzt, gefolgt von ACE-Hemmern bzw. AT1-Blockern (13\%) und Kalzium-Antagonisten (7,4\%). Die restlichen 3,5\% bildeten andere antiarrythmische bzw. blutdrucksenkende Medikamente, vorwiegend Kombinationspräparate mit Hydrochlorothiazid. Insgesamt nahmen über die Hälfte der Patientinnen (51,9\%) sonstige Medikamente, vor allem Schilddrüsentabletten, Protonenpumpenhemmer, Statine und die Antibabypille ein. Nur fünf Patientinnen (9,3\%) nahmen keinerlei Medikamente ein. Herzwirksame Medikamente, zu denen BBlocker, ACE-Hemmer, $\mathrm{Ca}^{2+}$-Antagonisten, andere Antihypertensiva sowie anticholinerg wirksame Medikamente gezählt wurden, nahmen insgesamt 24,1\% der Patientinnen ein. Der Einfluss dieser ggf. problematischen Medikamente auf die Stressreagibilität wurde von G. Bornschein in einer parallelen Arbeit untersucht. 
Tabelle 6: Verteilung eingenommener Medikamente

\begin{tabular}{|l|l|c|}
\hline $\begin{array}{l}\text { Medikamente } \\
\text { Gesamtkollektiv n=54 }\end{array}$ & $\begin{array}{l}\text { Häufigkeiten } \\
\text { (Prozente) }\end{array}$ \\
\hline keine Medikamente & gesamt & $5(9,3 \%)$ \\
\hline \multirow{4}{*}{ Antidepressiva } & gesamt & $39(72,2 \%)$ \\
\cline { 2 - 3 } & SSRI & $16(29,6 \%)$ \\
\cline { 2 - 3 } & Trizyklische (TZA) & $13(24,1 \%)$ \\
\cline { 2 - 3 } & andere & $21(38,9 \%)$ \\
\hline Neuroleptika & gesamt & $23(42,6 \%)$ \\
\hline \multirow{4}{*}{ Tranquilizer/Sedativa } & gesamt & $20(37,0 \%)$ \\
\cline { 2 - 3 } & Benzodiazepine & $18(33,3 \%)$ \\
\cline { 2 - 3 } Antihypertensiva & andere & $5(9,3 \%)$ \\
\hline Analgetika & gesamt & $8(14.8 \%)$ \\
\hline Antiarrhymika/ & gesamt & $13(24,1 \%)$ \\
\cline { 2 - 3 } & Betablocker & $7(14,8 \%)$ \\
\cline { 2 - 3 } & ACE-Hemmer/AT1- & $7(13,0 \%)$ \\
\cline { 2 - 3 } & Blocker & $4(7,4 \%)$ \\
\cline { 2 - 3 } & Ca2+-Antagonisten & $3(5,6 \%)$ \\
\cline { 2 - 3 } & andere & $28(51,9 \%)$ \\
\hline Sonstige Medikamente & gesamt &
\end{tabular}

\subsubsection{Psychometrischer Befund}

Tabelle 7 (Seite 46) zeigt die Ergebnisse (Mittelwerte mit Standardabweichung sowie minimale bzw. maximale angegebene Werte) der auf Station 9 eingesetzten Fragebögen mit spezifischen Subskalen zur Erstellung eines psychometrischen Befundes. Insgesamt füllten sechs Patientinnen des Gesamtkollektives ( $n=54)$ kein Fragebogen-Set aus. Von den verbleibenden 48 Teilnehmerinnen beantwortete jeweils eine Patientin den SCL-90-R bzw. den BDI-Fragebogen nicht, von vier Patientinnen fehlen die Daten des IES-R.

Der psychometrische Befund unseres Patientinnenkollektivs ist folgendermaßen zu charakterisieren:

Die Auswertung der Symptom-Checkliste (SCL-90-R) offenbart eine stark erhöhte grundsätzliche psychische Belastung (Global Severity Index (GSI): T-Wert=71). Auch die Subskalen Zwanghaftigkeit, Unsicherheit im Sozialkontakt, Depressivität und phobische Angst zeigen stark erhöhte T-Werte (>70). Die Subskalen Somatisierung, Ängstlichkeit und Psychotizismus haben deutlich erhöhte T-Werte (65-69), lediglich die Subskalen Aggressivität/Feindseligkeit sowie paranoides Denken sind nur leicht erhöht (T-Werte 60-64).

Die Patientinnen erzielten für den Fragebogen dissoziativer Symptome (FDS) einen Gesamtmittelwert von 19,6, welcher laut einer neueren ROC-Analyse von Bockers et al. (2008) als deutlich erhöht einzuschätzen ist. Bockers et al. (2008) ermittelten einen Cut-Off-Wert von 9,3 für den FDSGesamtwert.

Der Gesamtwert des Beck-Depressions-Inventars ist mit einem Mittelwert von 28,8 ( $\mathrm{SD}=10,80)$ deutlich erhöht und charakterisiert eine vorliegende depressive Episode als stark ausgeprägt. Dieser 
erhöhte Wert passt zu der im Gesamtkollektiv sehr oft gestellten Nebendiagnose einer depressiven

Störung.

Tabelle 7: Psychometrische Befunde der Patientinnen

\begin{tabular}{|c|c|c|c|c|c|c|}
\hline Psychometrische Skalen & $\mathbf{n}$ & $\begin{array}{c}\text { Mittel- } \\
\text { wert }\end{array}$ & $\begin{array}{l}\text { Standard- } \\
\text { abweichung }\end{array}$ & $\begin{array}{l}\text { Mini- } \\
\text { mum }\end{array}$ & $\begin{array}{l}\text { Maxi- } \\
\text { mum }\end{array}$ & $\begin{array}{c}\text { ggf. } \\
\text { T-Wert } \\
\text { bzw. } \\
\text { \%-Rang }\end{array}$ \\
\hline \multicolumn{7}{|l|}{ SCL-90-R } \\
\hline Somatisierung & 47 & 1,34 & 0,83 & 0 & 3,42 & 66 \\
\hline Zwanghaftigkeit & 47 & 1,91 & 0,89 & 0,20 & 3,60 & 73 \\
\hline Unsicherheit im Sozialkontakt & 47 & 1,94 & 1,00 & 0 & 3,88 & 72 \\
\hline Depressivität & 47 & 2,21 & 1,01 & 0 & 5,58 & 72 \\
\hline Ängstlichkeit & 47 & 1,85 & 0,93 & 0,30 & 3,50 & 69 \\
\hline Aggressivität/Feindseligkeit & 47 & 1,01 & 0,74 & 0 & 3,83 & 64 \\
\hline phobische Angst & 47 & 1,47 & 0,99 & 0 & 3,43 & 71 \\
\hline paranoides Denken & 47 & 1,27 & 0,83 & 0 & 3,17 & 63 \\
\hline Psychotizismus & 47 & 1,19 & 0,77 & 0 & 3,30 & 67 \\
\hline Global Severity Index & 47 & 1,64 & 0,68 & 0,29 & 2,97 & 71 \\
\hline \multicolumn{7}{|l|}{ FDS } \\
\hline FDS-Gesamtwert & 48 & 19,6 & 14,30 & 1,4 & 57,3 & \\
\hline DES-Gesamtwert & 48 & 22,6 & 16,46 & 2,1 & 62,5 & \\
\hline Dissoziative Amnesie & 48 & 11,1 & 12,64 & 0 & 48,8 & \\
\hline $\begin{array}{l}\text { Absorption (Tendenz zu } \\
\text { Imaginativen Erlebnisweisen) }\end{array}$ & 48 & 29,0 & 17,83 & 3,3 & 68,9 & \\
\hline Depersonalisation/Derealisation & 48 & 20,5 & 19,90 & 0 & 73,3 & \\
\hline $\begin{array}{l}\text { pseudoneurologische } \\
\text { Konversionssymptome }\end{array}$ & 48 & 14,2 & 13,35 & 0 & 51,1 & \\
\hline ICD-10 Symptome & 48 & 14,5 & 11,90 & 0 & 50,6 & \\
\hline \multicolumn{7}{|l|}{ IES-R } \\
\hline Intrusion & 44 & 24,64 & 7,59 & 8 & 35 & \\
\hline Vermeidung & 44 & 26,27 & 8,71 & 6 & 52 & \\
\hline Übererregung & 44 & 23,64 & 7,58 & 7 & 35 & \\
\hline \multicolumn{7}{|l|}{ BDI } \\
\hline Gesamtwert & 47 & 28,81 & 10,80 & 3 & 47 & \\
\hline \multicolumn{7}{|l|}{ BPI } \\
\hline Identitätsdiffusion & 48 & 6,25 & 3,46 & 0 & 12 & 58 \\
\hline Angst vor Nähe & 48 & 4,52 & 1,94 & 0 & 8 & 62 \\
\hline Primitive Abwehr & 48 & 4,25 & 2,46 & 0 & 13 & 57 \\
\hline mangelhafte Realitätsprüfung & 48 & 0,87 & 1,28 & 0 & 5 & 61 \\
\hline Cut-20 & 48 & 11,23 & 4,36 & 2 & 21 & 60 \\
\hline \multicolumn{7}{|l|}{ BSL } \\
\hline Selbstwahrnehmung & 48 & 25,17 & 15,13 & 3 & 64 & 40 \\
\hline Affektregulation & 48 & 25,33 & 11,85 & 7 & 49 & 34 \\
\hline Autoaggression & 48 & 16,90 & 11,50 & 0 & 44 & 31 \\
\hline Dysphorie & 48 & 31,83 & 5,14 & 21 & 40 & 45 \\
\hline soziale Isolation & 48 & 13,77 & 10,58 & 0 & 44 & 33 \\
\hline Intrusionen & 48 & 11,31 & 8,08 & 0 & 33 & 51 \\
\hline Feindseligkeit & 48 & 5,79 & 4,18 & 0 & 16 & 31 \\
\hline Gesamtskala & 48 & 152,40 & 64,49 & 56 & 290 & 28 \\
\hline
\end{tabular}


Die ermittelten Werte auf der Impact of Event Scale (IES-R) für die drei Subskalen Intrusion, Vermeidung und Übererregung entsprechen Vergleichswerten (Mittelwerte \pm Standardabweichung) von Frauen mit sexuellen Gewalterfahrungen und sind im Vergleich zu Nichttraumatisierten extrem erhöht.

Für das Borderline-Persönlichkeits-Inventar (BPI) wurde für den Cut-Off-Wert bestehend aus 20 Items (Cut-20) aus den Einzelwerten der Patientinnen ein Durchschnittswert für das Gesamtkollektiv von 11,23 ermittelt. Dieses Ergebnis unterstreicht die hohe Komorbidität einer BorderlinePersönlichkeitsstörung in unserem PTBS-Patientinnenkollektiv, denn bereits ab einem Cut-20-Wert von $\geq 10$ ist das Vorliegen einer Borderline-Persönlichkeitsstörung sehr wahrscheinlich. Die einzelnen Subskalen des BPI Identitätsdiffusion, Angst vor Nähe, primitive Abwehr sowie mangelhafte Realitätsprüfung zeigen insgesamt jedoch nur leicht erhöhte Werte (T-Werte 57-62).

Die Auswertung der Borderline-Symptom-Liste (BSL) erfolgt anhand von Prozenträngen, welche die Schwere der Symptombelastung im Vergleich zu einer Normierungsstichprobe bestehend aus Borderline-Patientinnen $(n=308)$ angeben. Unsere Patientinnen weisen in den Bereichen Affektregulation, Autoaggression, soziale Isolation sowie Feindseligkeit eine gleich große oder größere Symptombelastung als etwa ein Drittel der Normierungsstichprobe auf (Prozentränge 31-34). Eine ähnliche Stellung nimmt auch die Gesamtskala der BSL ein, mit einem Wert von 152,4 ergibt sich ein Prozentrang von 28. Für die Subskalen Selbstwahrnehmung, Dysphorie und insbesondere Intrusionen ergeben sich höhere Prozentränge (40-51) und damit eine stärkere Symptomausprägung.

\subsection{Subjektives Stressempfinden}

Im Rahmen des Ausfüllens eines allgemeinen Fragebogens zu soziodemographischen Daten wurden die Patientinnen auch gebeten, die Frage zu beantworten, ob sie sich in letzter Zeit besonders gestresst fühlten. Diesbezüglich gab ein Großteil der Patientinnen (70,4\%) an, sich besonders gestresst zu fühlen.

Betrachtet man das subjektive Stresserleben der Patientinnen, welches sie während der TFM-Messung auf einer Subjektivskala (Subject Units of Discomfort, SUD) von 0 bis 10 angegeben haben, so ergibt sich folgendes Bild (

Tabelle 8, Seite 48): Die Patientinnen gaben bereits in Ruhe ein Stressempfinden von durchschnittlich 3,3 ( $\mathrm{SD}=1,81)$ an, der Median lag bei 3,0. Beide Stresstests „Kopfrechnen“ und „Babyschreien“ führten bei den Patientinnen zu einer erwarteten, hochsignifikanten Zunahme des subjektiven Stressempfindens im Vergleich zum Ausgangsstressniveau in Ruhe (p-Wert jeweils <0,001). Die Entspannungsmusik zeigte mit einem Mittelwert von 4,2 bzw. einem Median von 4,0 nicht die erwartete Abnahme der Angaben auf der subjektiven Analogskala auf Ausgangsniveau, es bestand ein signifikanter Unterschied zwischen Baseline und Musik ( $\mathrm{p}<0,05)$. 
Tabelle 8: $\quad$ Subjektives Stressempfinden (Analogskala) während der Stressbelastungsuntersuchung (Rechnen, Babyschreien, Entspannungsmusik)

\begin{tabular}{|c|c|c|c|c|c|}
\hline $\begin{array}{c}\text { Subjektives } \\
\text { Stressempfinden } \\
\text { Gesamtkollektiv n=54 }\end{array}$ & Mittelwert & $\begin{array}{c}\text { Standard- } \\
\text { abweichung }\end{array}$ & Median & Minimum & Maximum \\
\hline $\begin{array}{c}\text { Ausgangswert in } \\
\text { Ruhe (Baseline) }\end{array}$ & 3,29 & 1,81 & 3,0 & 0 & 7,5 \\
\hline Kopfrechentest & 6,28 & 1,96 & 6,5 & 0 & 10,0 \\
\hline Babyschreien & 6,28 & 2,58 & 7,0 & 0 & 10,0 \\
\hline Entspannungsmusik & 4,21 & 2,96 & 4,0 & 0 & 10,0 \\
\hline
\end{tabular}

Der Verlauf des subjektiven Stresserlebens während der einzelnen Phasen der Stressbelastungsuntersuchung ist in der Abbildung 10 (Seite 48) dargestellt.

Im paarweisen Vergleich der beiden Stressoren zeigte sich kein signifikanter Unterschied, die Mittelwerte waren mit 6,3 identisch, lediglich der Median unterschied sich um 0,5.

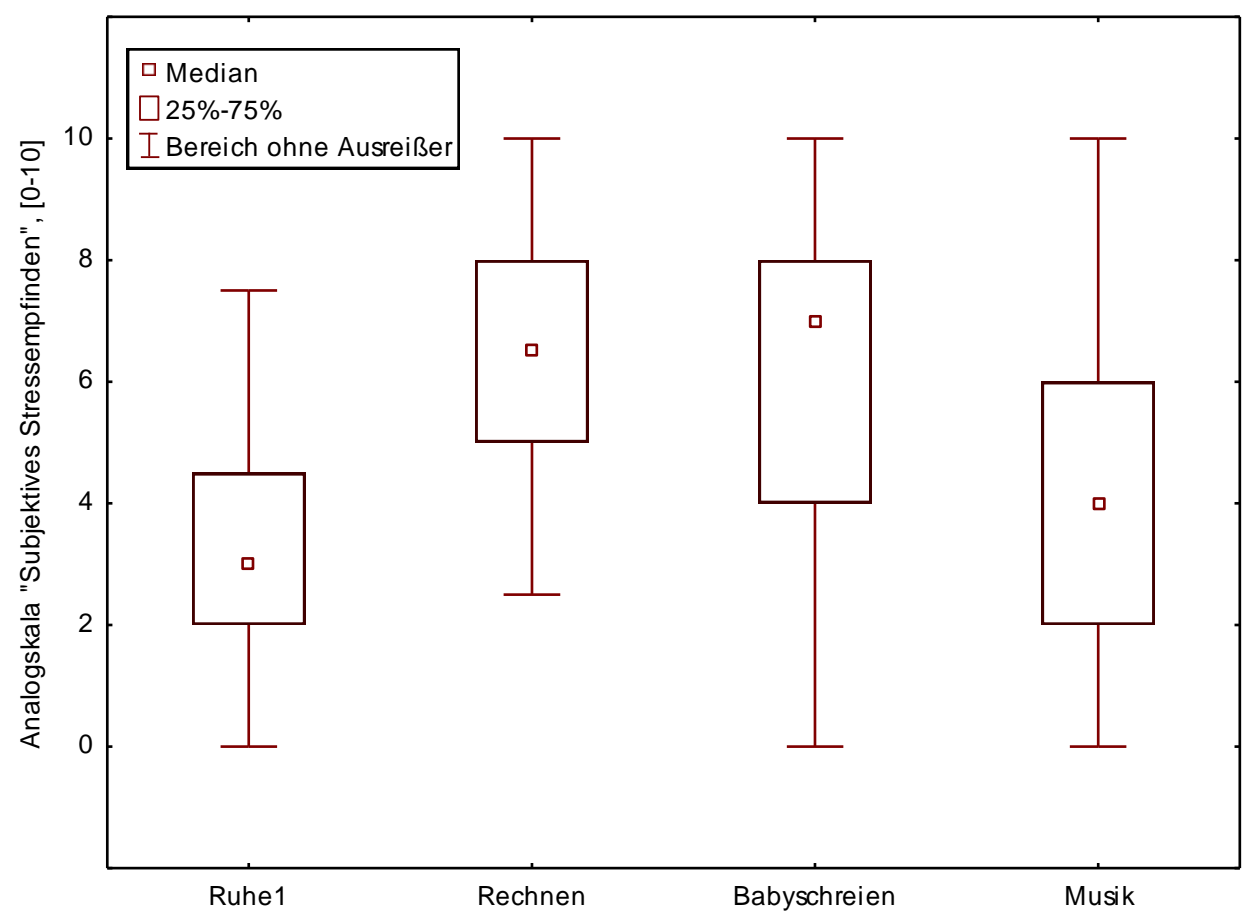

Abbildung 10: Angaben des subjektiven Stressempfindens (Analogskala: 0 =,gar kein Stress“ bis 10 $=$, ,schlimmster Stress $\left.{ }^{6}\right)$ während der Stressbelastungsuntersuchung - Verteilung als Whisker-Boxplot (Median, Quartile, Minimum, Maximum) für die einzelnen Messphasen 
Hinsichtlich der Häufigkeitsverteilung (Abbildung 11, Seite 49 und Abbildung 12, Seite 49) konnte jedoch festgestellt werden, dass das Babyschreien im Vergleich zum Rechnen die Patientinnen etwas häufiger veranlasste, den Maximalwert von 10 Punkten auf der Analogskala zu vergeben. 3,7 \% der Patientinnen empfanden das Rechnen als maximal stressvoll, während das Babyschreien sogar bei 9,3 \% der Patientinnen ein maximales Stressempfinden auslöste. Während des Babyschreiens zeigt sich im Vergleich zum Rechentest eine breitere Streuung der Angaben auf der SUD.

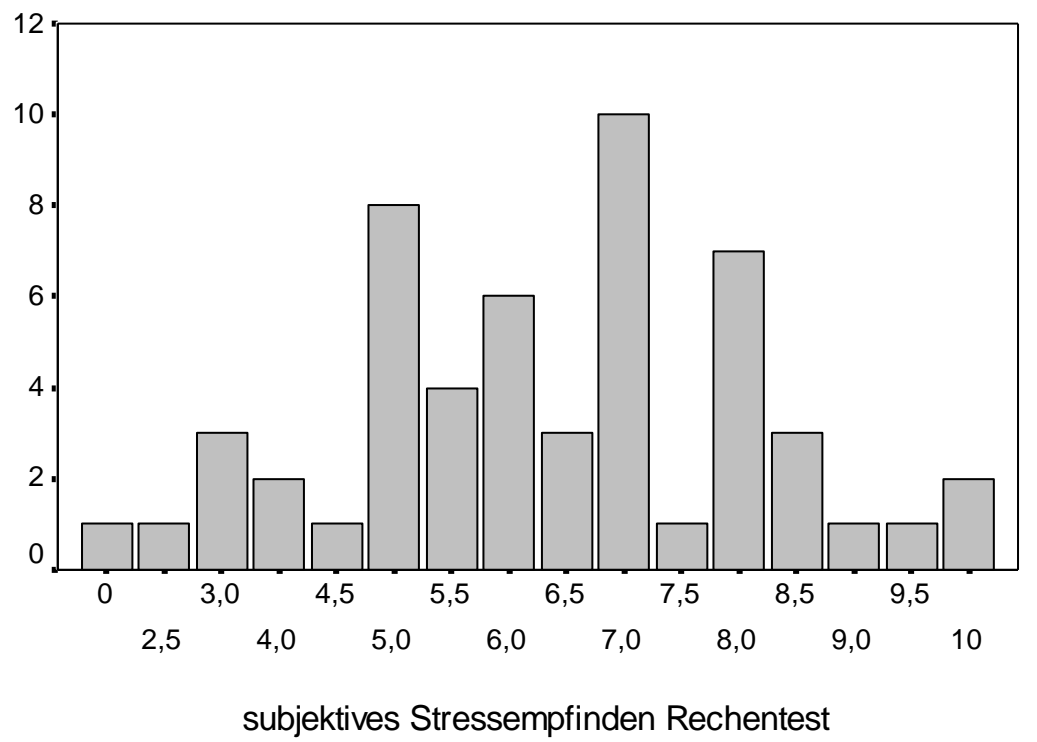

Abbildung 11: Häufigkeitsverteilung des subjektiven Stressempfindens (Analogskala 0-10) während des Kopfrechentests im Gesamtkollektiv $(n=54)$

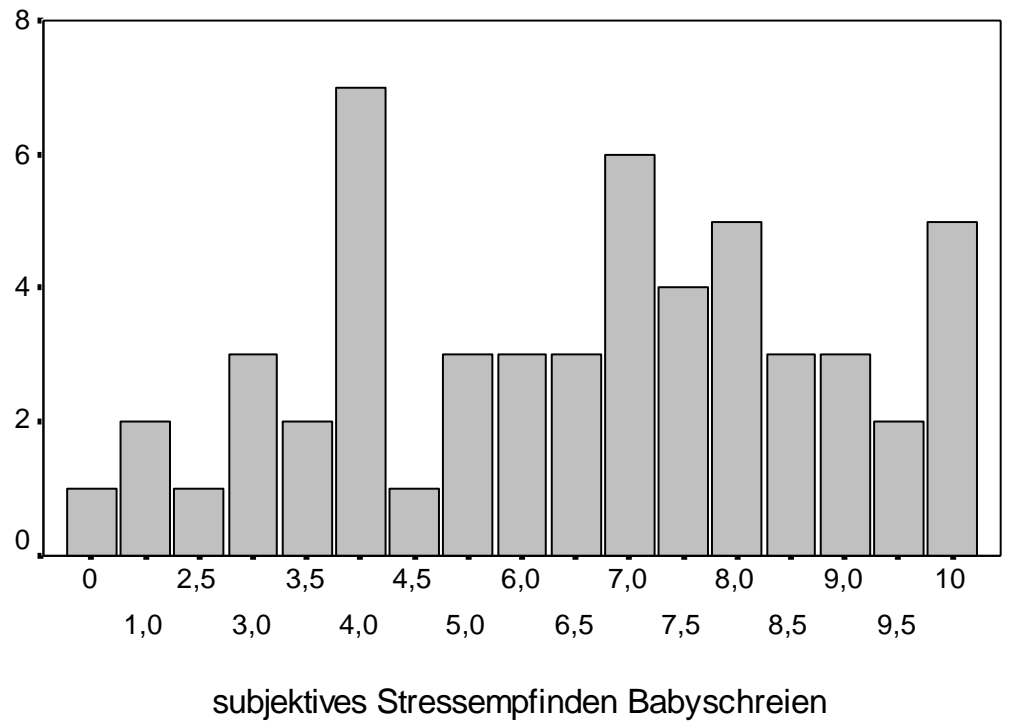

Abbildung 12: Häufigkeitsverteilung des subjektiven Stressempfindens (Analogskala 0-10) während des Babyschreiens im Gesamtkollektiv (n=54) 


\subsection{Beobachtbare emotionale bzw. vegetative Reaktionen}

Im Rahmen der Stressbelastungsuntersuchung zeigten einige Patientinnen beobachtbare emotionale bzw. vegetative Reaktionen in Form von schwerer Atmung, Erregungszuständen (Gänsehaut, Schwitzen, Herzklopfen, Zucken...), Weinen bzw. Tränen in den Augen, das Nutzen von Copingmechanismen (Coolpack, Stachelball, „Herunteratmen“...) oder Numbing-Verhalten (starrer Blick, verzögerte Reaktion auf Ansprechen...) (Tabelle 9, Seite 50).

Tabelle 9: Beobachtbare emotionale bzw. vegetative Reaktionen während der Stressbelastungstests

\begin{tabular}{|l|l|c|}
\hline $\begin{array}{l}\text { Emotionale/vegetative } \\
\text { Begleitsymptomatik } \\
\text { Gesamtkollektiv n=54 }\end{array}$ & $\begin{array}{c}\text { Häufigkeiten } \\
\text { (Prozente) }\end{array}$ \\
\hline Kopfrechentest & schweres Atmen & $2(3,7 \%)$ \\
\cline { 2 - 3 } & Erregungszustand & $4(7,4 \%)$ \\
\cline { 2 - 3 } & Weinen & $2(3,7 \%)$ \\
\cline { 2 - 3 } & Copingmechanismen & $3(5,6 \%)$ \\
\cline { 2 - 3 } & Numbing & $1(1,9 \%)$ \\
\cline { 2 - 3 } & Mundtrockenheit & $8(14,8 \%)$ \\
\cline { 2 - 3 } & Konzentrationsprobleme & $22(40,7 \%)$ \\
\hline \multirow{5}{*}{ Babyschreien } & schweres Atmen & $18(33,3 \%)$ \\
\cline { 2 - 3 } & Erregungszustand & $13(24,1 \%)$ \\
\cline { 2 - 3 } & Weinen & $6(11,1 \%)$ \\
\cline { 2 - 3 } & Copingmechanismen & $12(22,2 \%)$ \\
\cline { 2 - 3 } & Numbing & $6(11,1 \%)$ \\
\hline \multirow{5}{*}{ Entspannungsmusik } & schweres Atmen & $4(7,4 \%)$ \\
\cline { 2 - 3 } & Erregungszustand & $3(5,6 \%)$ \\
\cline { 2 - 3 } & Weinen & $2(3,7 \%)$ \\
\cline { 2 - 3 } & Copingmechanismen & $1(1,9 \%)$ \\
\cline { 2 - 3 } & Numbing & $2(3,7 \%)$ \\
\hline
\end{tabular}

Dabei konnten signifikante Unterschiede für einzelne Reaktionsformen im Vergleich von Kopfrechentest, Babyschreien und Entspannungsmusik festgestellt werden (Abbildung 13, Seite 51).

Die globale Analyse zeigte eine Signifikanz für schwere Atmung $(\mathrm{p}<0,001)$, Erregungszustände $(p<0,01)$ und das Nutzen von Copingstrategien $(p<0,01)$. Insbesondere das Babyschreien führte zu einer starken Aktivierung der Patientinnen mit Zunahme des Auftretens aller beschriebenen Reaktionsformen. Im paarweisen Vergleich von Rechnen und Babyschreien waren nach Adjustierung die $\mathrm{p}$-Werte von schwerer Atmung $(\mathrm{p}<0,001)$ und dem Nutzen von Copingmechanismen $(\mathrm{p}<0,05)$ signifikant unterschiedlich, für den Erregungszustand zeigte sich eine statistische Tendenz $(p=0,052)$. Vergleicht man das Reaktionsverhalten während des Rechentests mit den Reaktionen während der Entspannungsmusik, so zeigt sich kein signifikanter Unterschied. Jedoch gibt es einen signifikanten Unterschied in den Reaktionen während der Musik vs. Babyschreiens für das schwere Atmen $(\mathrm{p}<0,01)$, die Erregungszustände $(\mathrm{p}<0,05)$ und das Nutzen von Copingstrategien $(\mathrm{p}<0,01)$.

Während des Kopfrechentests beklagten acht Frauen (14,8\%) zusätzlich Mundtrockenheit bzw. „das Gefühl einen Kloß im Hals $\mathrm{zu}$ haben“ und 22 Patientinnen (40,7\%) hatten große Konzentrationsschwierigkeiten. 


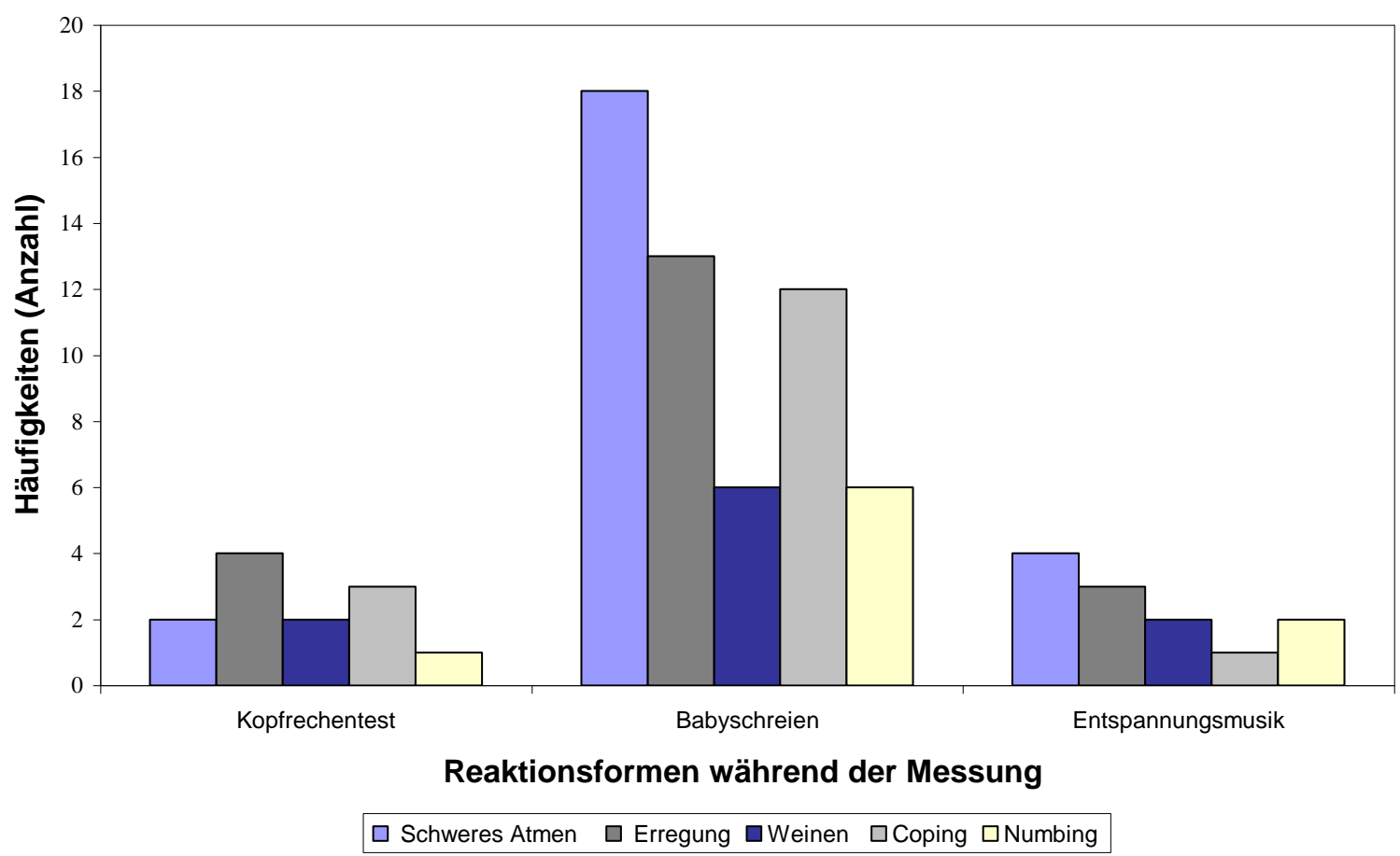

Abbildung 13: Reaktionsformen während der Stressbelastungsuntersuchung (Rechnen, Babyschreien und Entspannungsmusik)

$\mathrm{Da}$ die meisten vegetativen Reaktionen während des Babyschreiens beobachtet werden konnten, erfolgte für diese Phase eine Korrelationsanalyse, um einen Zusammenhang zwischen der Anzahl beobachteter Reaktionsformen und den physiologischen Parametern aufzudecken (Tabelle 10, Seite 51). Es zeigte sich eine signifikante Korrelation (Tau b) für die Herzfrequenz (HR) von 0,44 sowie negative signifikante Korrelationen für das Hochfrequenzband der Herzratenvariabilität (HF-HRV) von -0,36, das Niederfrequenzband der Herzratenvariabilität (LF-HRV) von $-0,30$ und die Barorezeptorsensitivität (BRS) von $-0,40$ (p-Wert jeweils <0,01). Für alle weiteren TFM-Parameter fielen die Korrelationskoeffizienten deutlich niedriger aus und waren nicht signifikant.

Tabelle 10: Korrelation nach Kendall-Tau-b zwischen der Anzahl der beobachteten vegetativen Reaktionen und TFM-Parametern während des Babyschreiens, Korrelationskoeffizient und ggf. Signifikanzniveau $(* \mathbf{p}<0,05, * * \mathbf{p}<0,01)$

\begin{tabular}{|c|c|c|c|c|c|c|c|c|c|}
\hline \multicolumn{7}{|c|}{$\begin{array}{c}\text { Korrelation der Anzahl beobachteter vegetativer Reaktionen } \\
\text { mit TFM-Parametern während des Babyschreiens }\end{array}$} \\
\hline $\begin{array}{c}\text { Korrelation } \\
\text { nach } \\
\begin{array}{c}\text { Kendall- } \\
\text { Tau-b }\end{array}\end{array}$ & HR & CI & TRPI & sBP & SDNN & $\begin{array}{c}\text { log HF- } \\
\text { HRV }\end{array}$ & $\begin{array}{c}\text { log LF- } \\
\text { HRV }\end{array}$ & $\begin{array}{c}\text { BRS } \\
\text { total }\end{array}$ \\
\cline { 2 - 8 } & \multicolumn{7}{|c|}{ Korrelationskoeffizient } \\
$\begin{array}{c}\text { Anzahl } \\
\text { vegetativer } \\
\text { Reaktionen }\end{array}$ & $0,438 * *$ & 0,010 & 0,038 & 0,088 & $-0,126$ & $-0,362 * *$ & $-0,307 * *$ & $-0,398 * *$ \\
\hline
\end{tabular}


Die als abschließende, beruhigende Musik ausgewählte Mondscheinsonate von Beethoven führte nicht bei allen Patientinnen zur erwünschten Entspannung, 19 Patientinnen empfanden die Musik sogar als unangenehm bzw. emotional aufwühlend und nicht entspannend (Abbildung 14, Seite 52).

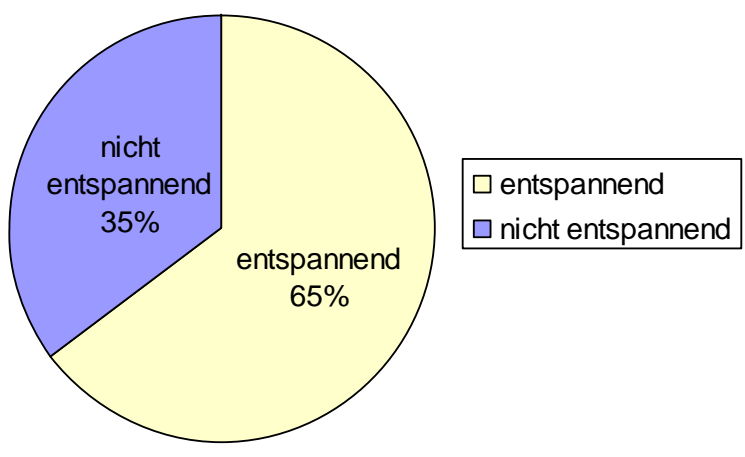

Abbildung 14: Patientinnen-Bewertung der Entspannungsmusik (n=54)

Das vorab unterbreitete Angebot, die Stressbelastungsuntersuchung jederzeit abbrechen zu können, nahmen einige Patientinnen wahr (Abbildung 15, Seite 52): eine Patientin (1,9\%) brach das Rechnen vorzeitig ab, für vier Patientinnen (7,4\%) war die Stressbelastung durch das Babyschreien zu groß und sechs Patientinnen $(11,1 \%)$ konnten unerwarteterweise die Musik nicht zu Ende hören. Die TFMMessung wurde dabei jeweils ohne Stressoreinwirkung bzw. Musikapplikation für fünf Minuten fortgesetzt. Nur bei einer Patientin musste die Messung auf Wunsch während der Musik verkürzt werden, sodass diese Phase nicht in die Auswertung einfließen konnte.

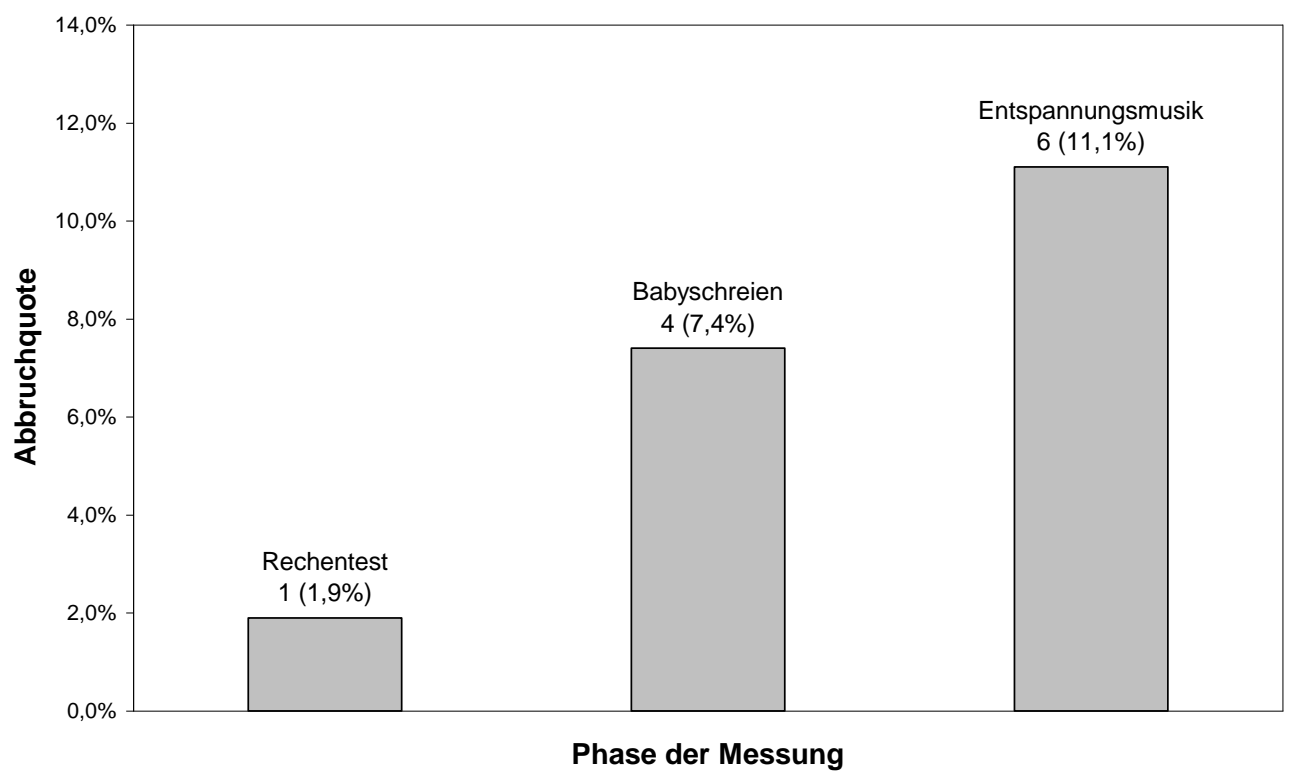

Abbildung 15: Abbruchquote im Gesamtkollektiv ( $n=54)$ für die einzelnen Stresstests während der Stressbelastungstests (Kopfrechnen, Babyschreien, Entspannungsmusik) 


\subsection{Auswertung physiologischer Stressparameter während der Stressbelastungsuntersuchung (Task-Force-Monitor-Messung)}

Zur Beurteilung der physiologischen Stressreagibilität auf hämodynamischer sowie autonomer Ebene wurden die fünf in den Hypothesen festgelegten TFM-Parameter Cardiac Index (CI), totaler peripherer Widerstands-Index (TPRI), Standardabweichung aller RR-Intervalle (SDNN) sowie zwei Komponenten der Herzratenvariabilität (HF- und LF-HRV) während der unterschiedlichen Phasen der Untersuchung betrachtet. Als weitere hämodynamische Parameter boten sich nach den Ergebnissen der globalen ANOVA-Analyse die Herzfrequenz (HR), als vorwiegend beta-adrenerg gesteuerter Parameter, sowie der Blutdruck (sBP, dBP und mBP), welcher vorwiegend alpha-adrenerg moduliert wird, für eine Verlaufsbetrachtung an. Um weitere Aussagen über die kardiale sympathovagale Balance treffen zu können, wurde zusätzlich zu den Parametern der Herzratenvariabilität (SDNN, HFHRV und LF-HRV) auch die Barorezeptorsensitivität (BRS) untersucht.

Zunächst soll der Verlauf dieser ausgewählten TFM-Parameter auf kollektiver Ebene dargestellt werden, dabei werden einzelne Phasen der Stressbelastungsuntersuchung miteinander verglichen. Ein besonderes Augenmerk liegt dabei auf dem Vergleich der beiden Stressoren „Rechnen“ und „Babyschreien“. Des Weiteren wird die Erholungsphase nach dem Babyschreien genauer untersucht, um die Rückbildungsfähigkeit der traumatisierten Frauen nach Stressanforderung besser beurteilen zu können.

\subsubsection{Verlauf der Task-Force-Monitor-Parameter auf kollektiver Ebene}

Die Phasenmittelwerte \pm Standardabweichung (SD), die Anzahl gültiger Werte [n] sowie die p-Werte der globalen einfaktoriellen ANOVA sind für die ausgewählten hämodynamischen und autonomen Parameter während der sechs Phasen der Stressbelastungsuntersuchung auf kollektiver Ebene in Tabelle 11 (Seite 54) zusammenfassend dargestellt. Im Folgenden sollen die physiologischen Parameter in ihrem Verlauf während der einzelnen Messphasen näher beschrieben werden, welcher mit Angaben der Phasenmittelwerte sowie des 95\%-Konfidenzintervalls für die spezifischen TFMParameter den Abbildung 16 bis Abbildung 23 zu entnehmen ist.

Anhand einer zunächst global durchgeführten einfaktoriellen repeated measures ANOVA konnten für die Parameter CI, HR, TPRI, sBP, dBP, mBP, SDNN, LF-HRV sowie BRS signifikante Unterschiede der Phasenmittelwerte zwischen den sechs Messphasen gefunden werden. Lediglich die HF-HRV zeigte keinen signifikanten Zeiteffekt. Im Anschluss an die globale Betrachtung folgte jeweils ein sechsfacher Paarvergleich der TFM-Parameter (Tabelle 12, Seite 55). Verglichen wurde die erste Ruhephase (Baseline) jeweils mit den beiden Stresstests (Kopfrechnen und Babyschreien), den Ruhephasen nach Stressoreinwirkung (Ruhe 2 und Ruhe 3) sowie mit der abschließenden Entspannungsmusik. Des Weiteren wurden die beiden Stressoren vergleichend gegenübergestellt. 
Tabelle 11: Hämodynamische (CI, HR, TPRI, sBP, dBP, mBP) und autonome (SDNN, HF-HRV, LFHRV, BRS) TFM-Parameter im Verlauf der Stressbelastungsuntersuchung; Mittelwerte \pm Standardabweichung, Anzahl [n], ggf. p-Wert der globalen ANOVA

\begin{tabular}{|c|c|c|c|c|c|c|c|}
\hline \multirow{2}{*}{$\begin{array}{c}\text { Task-Force- } \\
\text { Monitor- } \\
\text { Parameter }\end{array}$} & Ruhe 1 & $\begin{array}{l}\text { Rechen- } \\
\text { test }\end{array}$ & Ruhe 2 & $\begin{array}{c}\text { Baby- } \\
\text { schreien }\end{array}$ & Ruhe 3 & $\begin{array}{l}\text { Entspan- } \\
\text { nungs- } \\
\text { musik }\end{array}$ & \multirow[t]{2}{*}{ p-Wert } \\
\hline & \multicolumn{6}{|c|}{ Mittelwert \pm Standardabweichung $[\mathrm{n}]$} & \\
\hline \multicolumn{8}{|c|}{ Hämodynamisch: beta-adrenerg } \\
\hline$\underset{\left(1 / \mathrm{min}^{*} \mathrm{~m}^{2}\right)}{\mathbf{C I}}$ & $\begin{array}{c}2,90 \\
\pm 0,78 \\
{[51]}\end{array}$ & $\begin{array}{c}3,09 \\
\pm 0,80 \\
{[51]}\end{array}$ & $\begin{array}{c}2,87 \\
\pm 0,725 \\
{[51]}\end{array}$ & $\begin{array}{c}2,82 \\
\pm 0,743 \\
{[51]}\end{array}$ & $\begin{array}{c}2,86 \\
\pm 0,694 \\
{[51]}\end{array}$ & $\begin{array}{c}2,72 \\
\pm 0,686 \\
{[50]}\end{array}$ & $<0,001$ \\
\hline$\underset{\left(\mathrm{min}^{-1}\right)}{\mathbf{H R}}$ & $\begin{array}{c}76,39 \\
\pm 10,13 \\
{[52]} \\
\end{array}$ & $\begin{array}{c}79,51 \\
\pm 10,09 \\
{[52]} \\
\end{array}$ & $\begin{array}{c}75,65 \\
\pm 10,30 \\
{[52]} \\
\end{array}$ & $\begin{array}{c}75,31 \\
\pm 10,70 \\
{[52]} \\
\end{array}$ & $\begin{array}{c}75,06 \\
\pm 9,48 \\
{[52]} \\
\end{array}$ & $\begin{array}{c}73,35 \\
\pm 10,20 \\
{[51]}\end{array}$ & $<0,001$ \\
\hline \multicolumn{8}{|c|}{ Hämodynamisch: alpha-adrenerg } \\
\hline $\begin{array}{c}\text { TPRI } \\
\left(\mathrm{dyn} * \mathrm{~s} * \mathrm{~m}^{2} / \mathrm{cm}^{5}\right)\end{array}$ & $\begin{array}{c}2497,86 \\
\pm 763,68 \\
{[52]}\end{array}$ & $\begin{array}{c}2506,57 \\
\pm 804,83 \\
{[52]}\end{array}$ & $\begin{array}{c}2624,14 \\
\pm 805,16 \\
{[52]}\end{array}$ & $\begin{array}{c}2670,41 \\
\pm 895,26 \\
{[52]}\end{array}$ & $\begin{array}{c}2588,14 \\
\pm 757,02 \\
{[52]}\end{array}$ & $\begin{array}{c}2723,02 \\
\pm 861,59 \\
{[51]}\end{array}$ & $<0,001$ \\
\hline $\begin{array}{c}\mathbf{s B P} \\
(\mathrm{mmHg})\end{array}$ & $\begin{array}{c}114,44 \\
\pm 12,28 \\
{[52]} \\
\end{array}$ & $\begin{array}{c}121,98 \\
\pm 13,83 \\
{[52]} \\
\end{array}$ & $\begin{array}{c}119,57 \\
\pm 14,75 \\
{[52]} \\
\end{array}$ & $\begin{array}{c}118,43 \\
\pm 17,37 \\
{[52]}\end{array}$ & $\begin{array}{c}118,25 \\
\pm 14,14 \\
{[52]} \\
\end{array}$ & $\begin{array}{c}117,64 \\
\pm 14,73 \\
{[51]}\end{array}$ & $<0,001$ \\
\hline$\underset{(\mathrm{mmHg})}{\mathbf{d B P}}$ & $\begin{array}{c}74,95 \\
\pm 9,00 \\
{[52]} \\
\end{array}$ & $\begin{array}{c}79,41 \\
\pm 10,38 \\
{[52]} \\
\end{array}$ & $\begin{array}{c}77,71 \\
\pm 10,40 \\
{[52]} \\
\end{array}$ & $\begin{array}{c}77,39 \\
\pm 12,43 \\
{[52]}\end{array}$ & $\begin{array}{c}77,00 \\
\pm 9,07 \\
{[52]}\end{array}$ & $\begin{array}{c}77,12 \\
\pm 10,16 \\
{[51]}\end{array}$ & $<0,01$ \\
\hline$\underset{(\mathrm{mmHg})}{\mathbf{m B P}}$ & $\begin{array}{c}86,68 \\
\pm 9,69 \\
{[52]}\end{array}$ & $\begin{array}{c}92,61 \\
\pm 10,96 \\
{[52]}\end{array}$ & $\begin{array}{c}90,59 \\
\pm 11,98 \\
{[52]}\end{array}$ & $\begin{array}{c}89,80 \\
\pm 14,41 \\
{[52]}\end{array}$ & $\begin{array}{c}89,38 \\
\pm 10,90 \\
{[52]}\end{array}$ & $\begin{array}{c}89,00 \\
\pm 11,54 \\
{[51]}\end{array}$ & $<0,01$ \\
\hline \multicolumn{8}{|c|}{ Autonom: Time-Domain } \\
\hline $\begin{array}{c}\text { SDNN } \\
(\mathrm{ms})\end{array}$ & $\begin{array}{c}54,07 \\
\pm 34,56 \\
{[52]}\end{array}$ & $\begin{array}{c}49,43 \\
\pm 22,84 \\
{[52]}\end{array}$ & $\begin{array}{c}63,57 \\
\pm 30,12 \\
{[52]}\end{array}$ & $\begin{array}{c}48,22 \\
\pm 30,37 \\
{[52]}\end{array}$ & $\begin{array}{c}60,58 \\
\pm 33,95 \\
{[52]}\end{array}$ & $\begin{array}{c}43,658 \\
\pm 21,592 \\
{[51]}\end{array}$ & $<0,001$ \\
\hline \multicolumn{8}{|c|}{ Autonom: Frequency-Domain } \\
\hline$\underset{\left(\mathrm{ms}^{2}\right)}{\log \mathbf{H F}-\mathbf{H R V}}$ & $\begin{array}{c}4,84 \\
\pm 1,25 \\
{[52]} \\
\end{array}$ & $\begin{array}{c}4,74 \\
\pm 1,24 \\
{[52]} \\
\end{array}$ & $\begin{array}{c}4,84 \\
\pm 1,23 \\
{[52]} \\
\end{array}$ & $\begin{array}{c}4,85 \\
\pm 1,37 \\
{[52]} \\
\end{array}$ & $\begin{array}{c}4,88 \\
\pm 1,30 \\
{[52]} \\
\end{array}$ & $\begin{array}{c}4,91 \\
\pm 1,32 \\
{[51]} \\
\end{array}$ & n.s. \\
\hline $\begin{array}{c}\log \mathbf{L F}-\mathbf{H R V} \\
\left(\mathrm{ms}^{2}\right)\end{array}$ & $\begin{array}{c}5,29 \\
\pm 1,20 \\
{[52]}\end{array}$ & $\begin{array}{c}5,70 \\
\pm 1,13 \\
{[52]}\end{array}$ & $\begin{array}{c}5,78 \\
\pm 1,05 \\
{[52]}\end{array}$ & $\begin{array}{c}5,22 \\
\pm 1,07 \\
{[52]}\end{array}$ & $\begin{array}{c}5,69 \\
\pm 1,00 \\
{[52]}\end{array}$ & $\begin{array}{c}5,29 \\
\pm 1,05 \\
{[51]}\end{array}$ & $<0,001$ \\
\hline \multicolumn{8}{|c|}{ Autonom: Barorezeptorsensitivität } \\
\hline $\begin{array}{l}\text { BRS total } \\
(\mathrm{ms} / \mathrm{mmHg})\end{array}$ & $\begin{array}{c}11,11 \\
\pm 7,32 \\
{[52]} \\
\end{array}$ & $\begin{array}{c}9,92 \\
\pm 5,15 \\
{[52]} \\
\end{array}$ & $\begin{array}{c}10,70 \\
\pm 6,24 \\
{[52]} \\
\end{array}$ & $\begin{array}{c}12,03 \\
\pm 7,32 \\
{[52]} \\
\end{array}$ & $\begin{array}{c}10,51 \\
\pm 5,54 \\
{[52]}\end{array}$ & $\begin{array}{c}13,29 \\
\pm 9,41 \\
{[51]}\end{array}$ & $<0,001$ \\
\hline
\end{tabular}


Tabelle 12: $\quad$ Sechsfacher Paarvergleich (Ruhe 1 vs. Rechnen/ Ruhe 2/ Babyschreien/ Musik, Rechnen vs. Babyschreien) der in der globalen ANOVA signifikanten TFM-Parameter (CI, HR, TPRI, sBP, dBP, mBP, SDNN, LF-HRV, BRS) für das Gesamtkollektiv [n=52], Phasenmittelwert-Differenzen \pm Standardfehler sowie ggf. p-Wert des t-Tests

\begin{tabular}{|c|c|c|c|c|c|c|}
\hline \multirow{2}{*}{$\begin{array}{c}\text { Task-Force- } \\
\text { Monitor- } \\
\text { Parameter }\end{array}$} & $\begin{array}{c}\text { Ruhe } 1 \\
\text { vs. } \\
\text { Rechnen }\end{array}$ & $\begin{array}{c}\text { Ruhe } 1 \\
\text { vs. } \\
\text { Ruhe } 2\end{array}$ & $\begin{array}{c}\text { Ruhe } 1 \\
\text { vs. Baby- } \\
\text { schreien }\end{array}$ & $\begin{array}{c}\text { Ruhe } 1 \\
\text { vs. } \\
\text { Ruhe } 3\end{array}$ & $\begin{array}{l}\text { Rechnen } \\
\text { vs. Baby- } \\
\text { schreien }\end{array}$ & $\begin{array}{c}\text { Ruhe } 1 \\
\text { vs. } \\
\text { Musik }\end{array}$ \\
\hline & \multicolumn{6}{|c|}{$\begin{array}{l}\text { Phasenmittelwert }- \text { Differenzen } \pm \text { Standardfehler } \\
\text { sowie p-Wert bei signifikantem Paarvergleich (adjustiert) }\end{array}$} \\
\hline \multicolumn{7}{|c|}{ Hämodynamisch: beta-adrenerg } \\
\hline$\underset{\left(1 / \min ^{*} \mathrm{~m}^{2}\right)}{\mathbf{C I}}$ & $\begin{array}{c}0,191 \\
\pm 0,032 \\
\mathrm{p}<0,001 \\
\end{array}$ & n.s. & n.s. & n.s. & $\begin{array}{c}-0,228 \\
\pm 0,054 \\
p<0,001\end{array}$ & n.s. \\
\hline $\begin{array}{c}\text { HR } \\
\left(\min ^{-1}\right)\end{array}$ & $\begin{array}{c}3,104 \\
\pm 0,487 \\
\mathrm{p}<0,001\end{array}$ & n.s. & n.s. & n.s. & $\begin{array}{c}-4,495 \\
\pm 0,700 \\
\mathrm{p}<0,001\end{array}$ & $\begin{array}{c}-2,930 \\
\pm 0,593 \\
p<0,001\end{array}$ \\
\hline \multicolumn{7}{|c|}{ Hämodynamisch: alpha-adrenerg } \\
\hline $\begin{array}{c}\text { TPRI } \\
\left(\mathrm{dyn} * \mathrm{~s} * \mathrm{~m}^{2} / \mathrm{cm}^{5}\right)\end{array}$ & n.s. & $\begin{array}{l}123,360 \\
\pm 30,871 \\
p<0,001\end{array}$ & $\begin{array}{c}174,551 \\
\pm 62,694 \\
\mathrm{p}<0.05 \\
\end{array}$ & n.s. & $\begin{array}{c}165,631 \\
\pm 60,075 \\
p<0.05 \\
\end{array}$ & $\begin{array}{l}209,261 \\
\pm 41,725 \\
p<0,001\end{array}$ \\
\hline $\begin{array}{c}\mathbf{S B P} \\
(\mathrm{mmHg})\end{array}$ & $\begin{array}{c}7,423 \\
\pm 1,202 \\
\mathrm{p}<0,001\end{array}$ & $\begin{array}{c}4,579 \\
\pm 1,094 \\
p<0,001\end{array}$ & n.s. & n.s. & n.s. & n.s. \\
\hline $\begin{array}{c}\mathbf{d B P} \\
(\mathrm{mmHg})\end{array}$ & $\begin{array}{c}4,528 \\
\pm 0,701 \\
p<0.0006\end{array}$ & $\begin{array}{c}2,470 \\
\pm 0,762 \\
\mathrm{p}=0.0072\end{array}$ & n.s. & n.s. & n.s. & n.s. \\
\hline $\begin{array}{c}\mathbf{m B P} \\
(\mathrm{mmHg})\end{array}$ & $\begin{array}{c}5,876 \\
\pm 0,824 \\
p<0.0006\end{array}$ & $\begin{array}{c}3,451 \\
\pm 0,985 \\
\mathrm{p}=0.0036\end{array}$ & n.s. & n.s. & n.s. & n.s. \\
\hline \multicolumn{7}{|l|}{ Autonom } \\
\hline $\begin{array}{l}\text { SDNN } \\
(\mathrm{ms})\end{array}$ & n.s. & n.s. & n.s. & n.s. & n.s. & n.s. \\
\hline$\underset{\left(\mathrm{ms}^{2}\right)}{\log \mathbf{L F}-\mathbf{H R V}}$ & $\begin{array}{c}0,41 \\
\pm 0,09 \\
\mathrm{p}<0,001\end{array}$ & $\begin{array}{c}0,49 \\
\pm 0,07 \\
\mathrm{p}<0,001\end{array}$ & n.s. & $\begin{array}{c}0,40 \\
\pm 0,08 \\
\mathrm{p}<0,001\end{array}$ & $\begin{array}{c}0,47 \\
\pm 0,10 \\
\mathrm{p}<0,001\end{array}$ & n.s. \\
\hline $\begin{array}{l}\text { BRS total } \\
(\mathrm{ms} / \mathrm{mmHg})\end{array}$ & n.s. & n.s. & n.s. & n.s. & $\begin{array}{c}2,150 \\
\pm 0,567 \\
p<0,01\end{array}$ & $\begin{array}{c}2,226 \\
\pm 0,595 \\
p<0,01\end{array}$ \\
\hline
\end{tabular}

\subsubsection{Cardiac Index (CI)}

Abbildung 16 (Seite 56) zeigt den Verlauf des CI während der einzelnen Phasen der Stressbelastungsuntersuchung. Die Paarvergleiche der einzelnen Untersuchungsabschnitte sind in Tabelle 12 (Seite 55) dargestellt. Der CI stieg vom Ausgangswert (Ruhe 1) zum Kopfrechentest im Mittel von 2,9 1/min*m² auf 3,1 1/min*m² (Phasenmittelwert-Differenz 0,2 1/min*m²), wobei dieser Anstieg auch im Paarvergleich statistische Signifikanz erreichte $(\mathrm{p}<0,001)$. Während der Erholungsphase nach dem Rechnen (Ruhe 2) fiel der CI sogar unter Ausgangsniveau und blieb auch während des Babyschreiens mit 2,8 1/min*m² unterhalb dieses Wertes. In der dritten Ruhephase nach dem Babyschreien konnte ein minimaler Anstieg auf 2,9 1/min*m² verzeichnet werden, bevor der CI 
während der Entspannungsmusik erwartungsgemäß auf das Minimum von 2,7 1/min*m² absank. Im Paarvergleich zeigte sich weiterhin ein signifikanter Unterschied des CI $(p<0,001)$ zwischen den beiden Stressoren „Rechnen“ und „Babyschreien“ mit einer Phasenmittelwert-Differenz von 0,2 $1 / \mathrm{min}^{*} \mathrm{~m}^{2}$

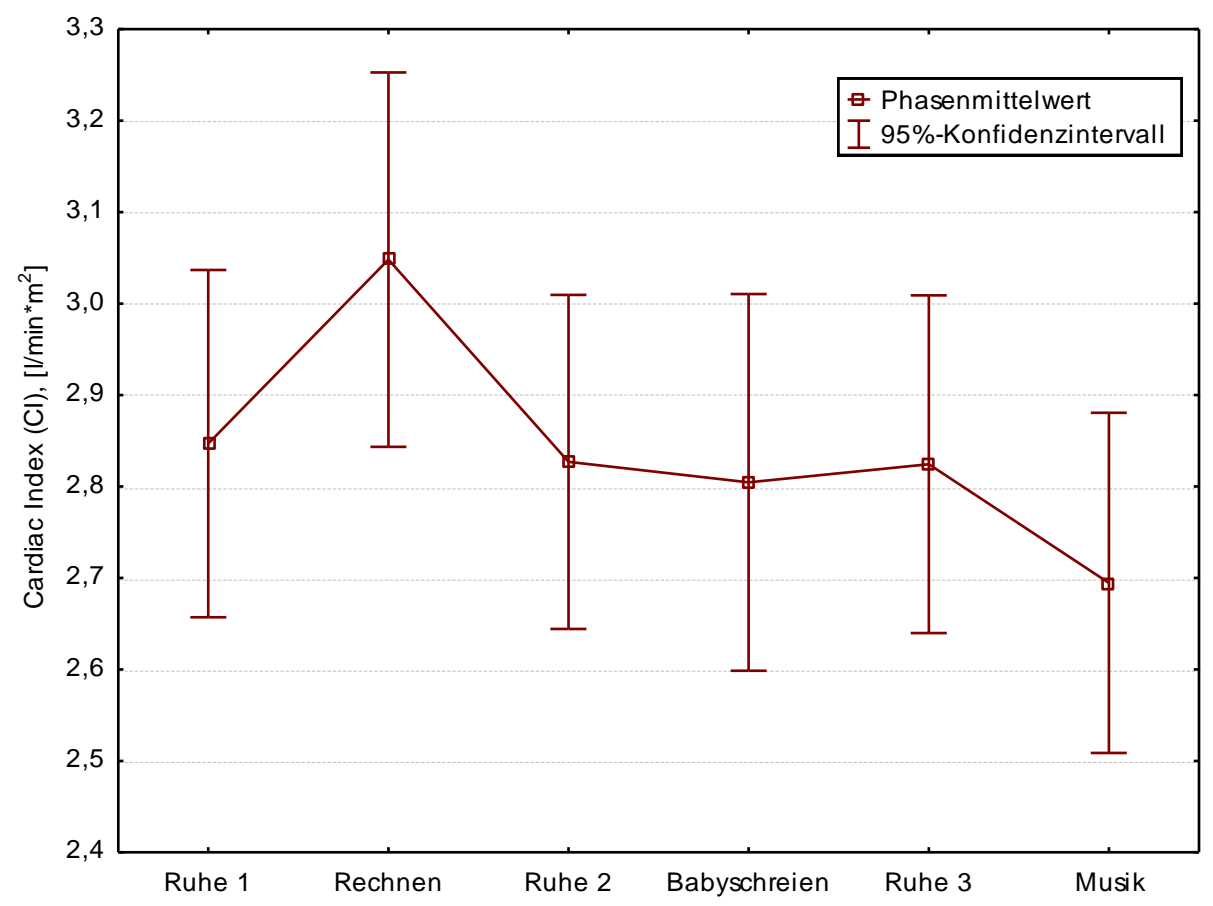

Abbildung 16: Verlauf des Cardiac Index (CI) während der Stressbelastungsuntersuchung, Phasenmittelwerte und 95\%-Konfidenzintervalle

\subsubsection{Herzfrequenz $(\mathrm{HR})$}

Der Verlauf der HR während der Stressbelastungsuntersuchung ist in Abbildung 17 (Seite 57) graphisch dargestellt. Tabelle 12 (Seite 55) zeigt die Ergebnisse der Phasenvergleiche. Die PTBSPatientinnen hatten bereits zu Beginn der Messung unter Ruhebedingungen eine mittlere HR von 76,4 /min, welche während des Rechentests erwartungsgemäß auf den höchsten Phasenmittelwert von 79,5 /min anstieg. Alle weiteren Phasen lagen im Mittel unterhalb des Ausgangswertes (Ruhe 1), wobei während der Entspannungsmusik das Minimum von 73,4/min erreicht wurde. Das Babyschreien führte nicht zu einem Anstieg der HR. Im Paarvergleich zeigte sich sowohl ein signifikanter Unterschied (p-Wert jeweils <0,001) zwischen Baseline und Rechnen (Phasenmittelwert-Differenz 3,1 /min), Baseline und Entspannungsmusik (Phasenmittelwert-Differenz -2,9/min) als auch zwischen den beiden Stressoren „Rechnen“ und „Babyschreien“ (Phasenmittelwert-Differenz -4,5/min). 


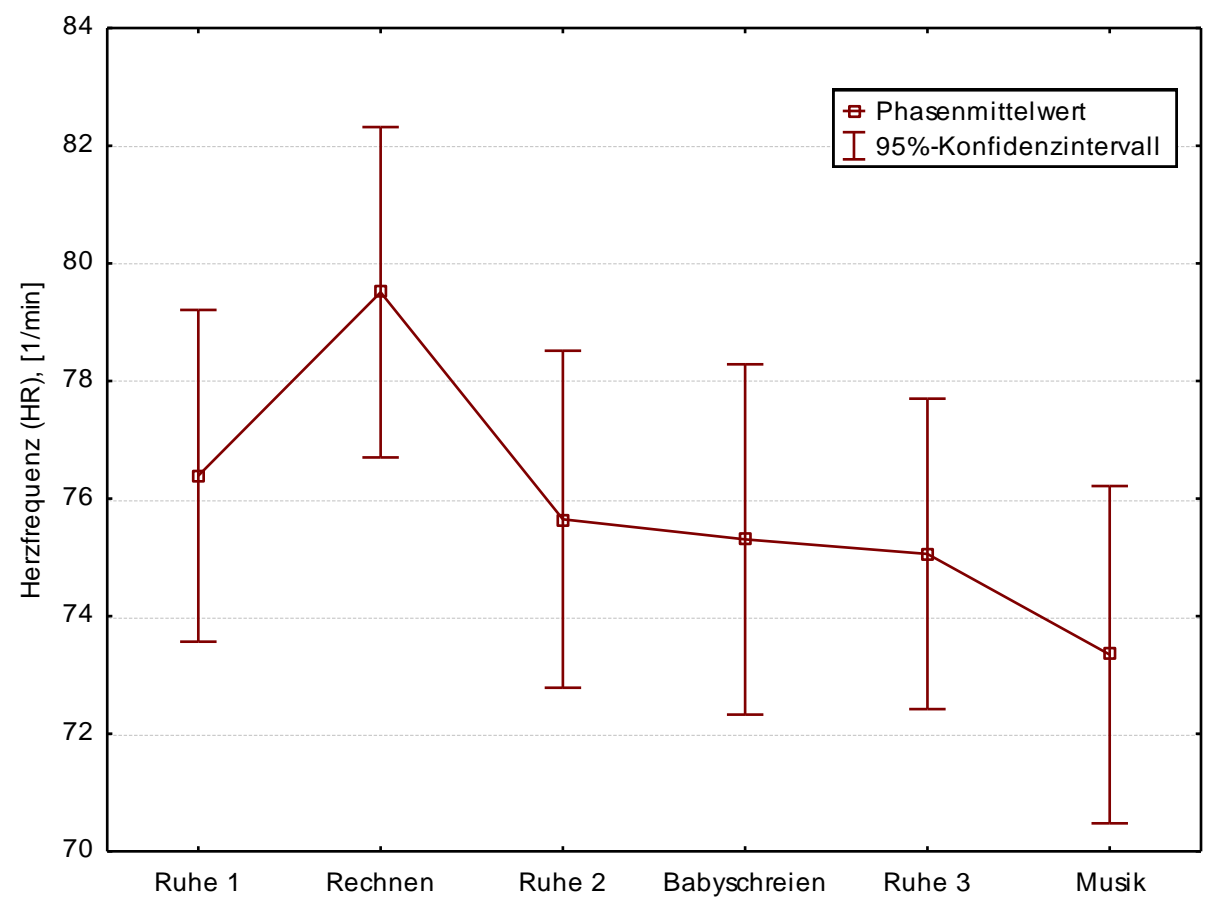

Abbildung 17:

Verlauf der Herzfrequenz (HR) während der Stressbelastungsuntersuchung, Phasenmittelwerte und 95\%-Konfidenzintervalle

\subsubsection{Totaler peripherer Widerstands-Index (TPRI)}

Der TPRI ist in seinem Verlauf in Abbildung 18 (Seite 57) dargestellt. Die einzelnen Phasenvergleiche sind Tabelle 12(Seite 55) zu entnehmen.

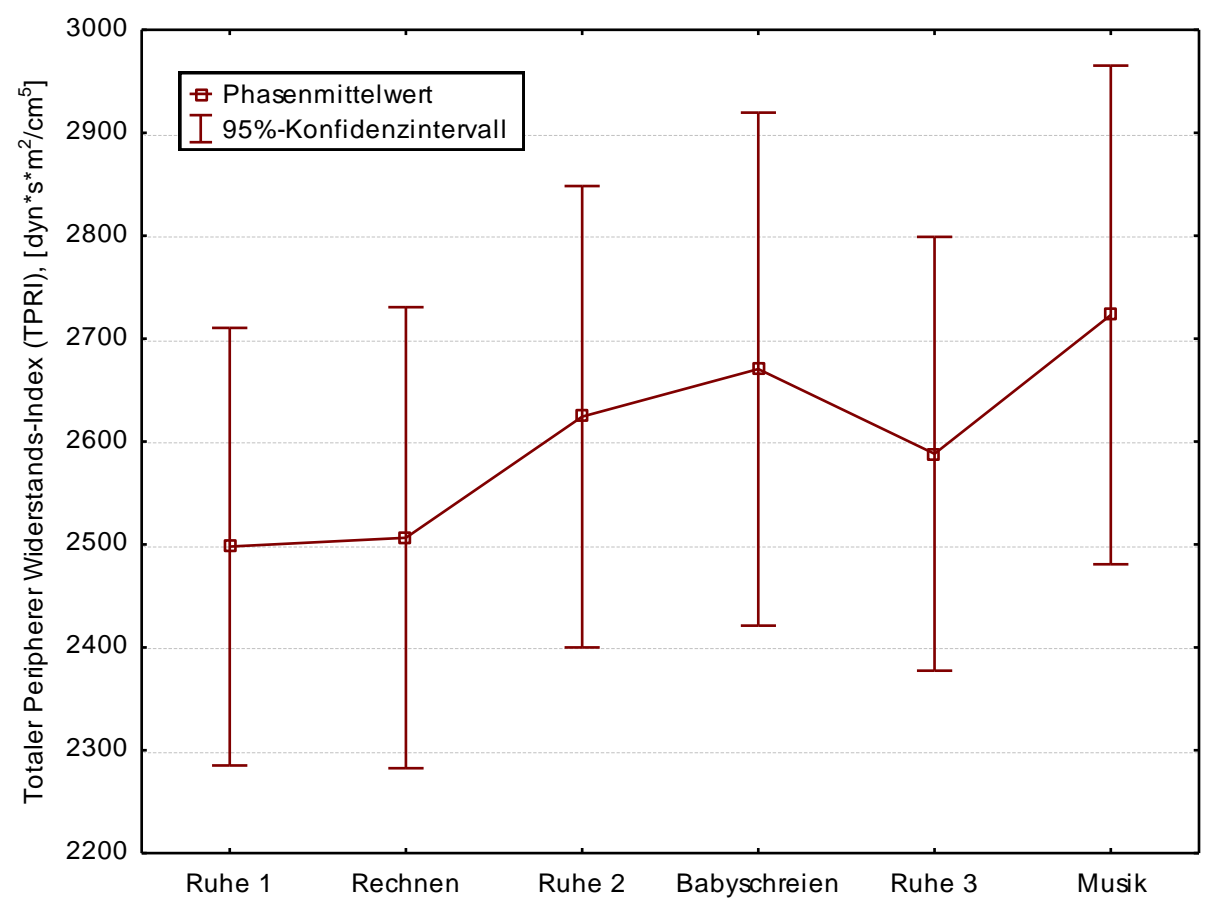

Abbildung 18: Verlauf des Totalen peripheren Widerstands-Index (TPRI) während der Stressbelastungsuntersuchung, Phasenmittelwerte und 95\%-Konfidenzintervalle 
Es zeigte sich ein Anstieg des Baseline-Wertes des TPRI $\left(2497,9\right.$ dyn* $\left.{ }^{*} \mathrm{~m}^{2} / \mathrm{cm}^{5}\right)$ sowohl während des Rechentests auf 2506,6 dyn*s* $\mathrm{m}^{2} / \mathrm{cm}^{5}$ als auch beim Babyschreien auf $2670,4 \mathrm{dyn} * \mathrm{~s} * \mathrm{~m}^{2} / \mathrm{cm}^{5}$, wobei lediglich der Einfluss des Babyschreiens mit einer Phasenmittelwert-Differenz von 174,6 dyn*s* ${ }^{2} / \mathrm{cm}^{5}$ statistisch signifikant war $(\mathrm{p}<0,05)$. Im Paarvergleich ergab sich daher ein signifikant unterschiedlicher Effekt der beiden Stressoren auf den TPRI $(p<0,05)$. Interessanterweise erreichte der TPRI seinen maximalen Phasenmittelwert mit 2723,0 dyn*s* $\mathrm{m}^{2} / \mathrm{cm}^{5}$ während der Entspannungsmusik, sodass auch hier ein signifikanter Unterschied zum Ausgangswert (Ruhe1) verzeichnet werden konnte $(\mathrm{p}<0,001)$.

\subsubsection{Systolischer, diastolischer und mittlerer arterieller Blutdruck (sBP, dBP, mBP)}

Hinsichtlich der drei Blutdruckmessgrößen (sBP, dBP und mBP) konnte eine Zunahme in allen Phasen im Vergleich zur Ruhe 1 festgestellt werden (Abbildung 19, Seite 58). Die einzelnen Paarvergleiche sind in Tabelle 12 (Seite 55) dargestellt. Der Kopfrechentest rief dabei den stärksten Effekt hervor. Stellvertretend für die drei Blutdruckmesswerte soll hier der sBP näher beschrieben werden. Dieser stieg im Mittel von 114,4 mmHg in der Ruhephase 1 auf 122,0 mmHg während des Kopfrechnens an (Phasenmittelwert-Differenz 7,4 mmHg). Die anderen zwei Blutdruckmessgrößen zeigten einen vergleichbaren Anstieg während des Rechentests. Der Paarvergleich erbrachte für diesen Stresstest für alle drei Blutdruckmessgrößen einen signifikanten Unterschied im Vergleich zum Ausgangswert in der Ruhephase 1 (p-Wert jeweils <0,001).

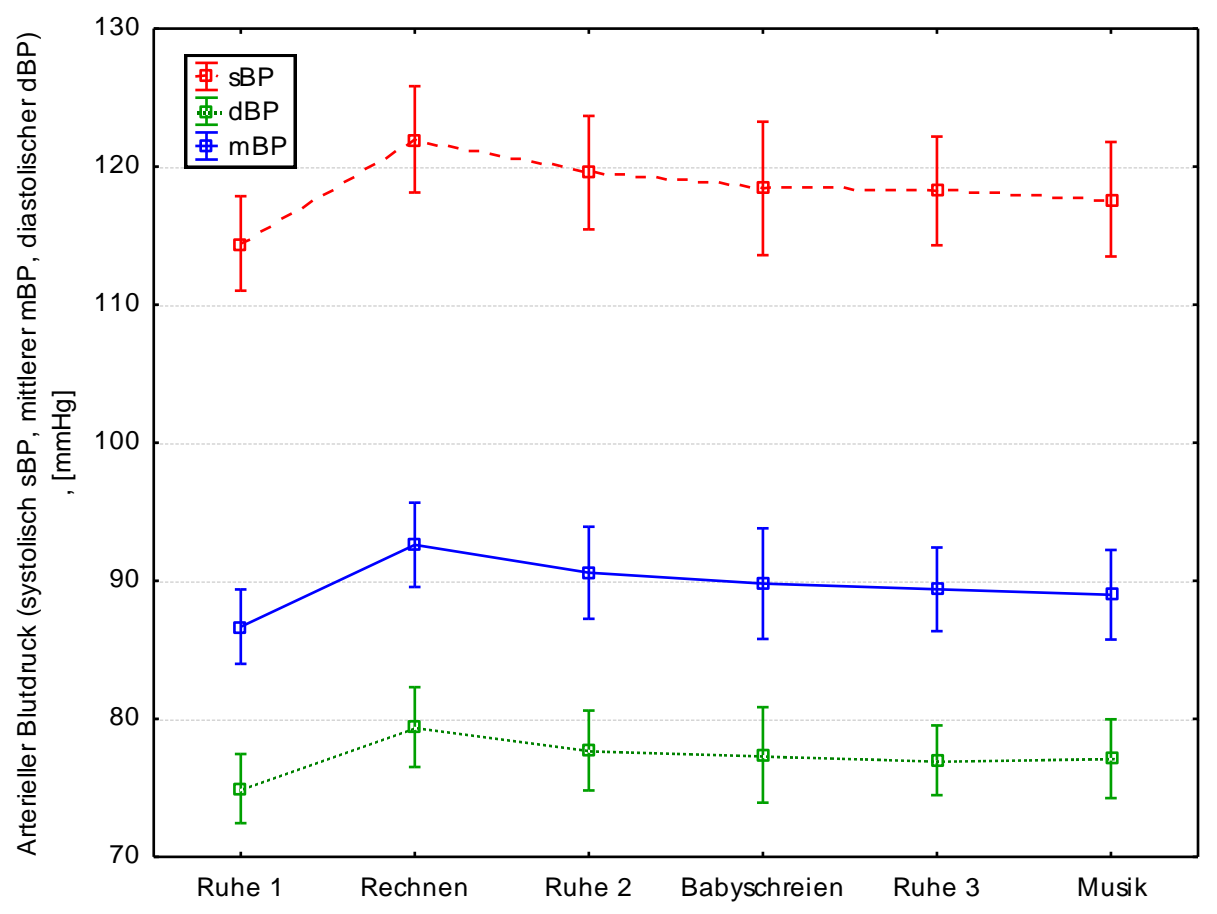

Abbildung 19: Verlauf des systolischen, diastolischen und mittleren Blutdrucks (sBP, dBP, mBP) während der Stressbelastungsuntersuchung, Phasenmittelwerte und 95\%Konfidenzintervalle 
Nach der Stressanforderung „Rechnen“ blieben alle drei Blutdruckmessgrößen weiterhin signifikant erhöht. Das Babyschreien führte unerwarteterweise zu keinem erneuten Anstieg des sBP (118,4 $\mathrm{mmHg}$ ) und folglich zu keinem signifikanten Paarvergleich mit den Werten der Ruhephase 1. Während der Entspannungsmusik sank der sBP zwar weiterhin leicht ab (117,6 mmHg), jedoch wurde der Ausgangswert (Ruhe 1) nicht erreicht.

\subsubsection{Standardabweichung aller RR-Intervalle (SDNN)}

Für die SDNN, ein Maß für die Gesamtvariabilität der HRV, konnte für beide Stresstests eine deutliche Abnahme vom Ausgangswert (54,07 ms) auf 49,43 ms beim Rechnen und 48,22 ms beim Babyschreien festgestellt werden (Abbildung 20, Seite 59). Im Gegensatz dazu stieg die SDNN in den Erholungsphasen nach Stressanforderung (Ruhe 2 und Ruhe 3) auf Werte über $60 \mathrm{~ms}$ an. Während der Entspannungsmusik fiel die SDNN auf einen minimalen Phasenmittelwert von 43,66 ms ab. Trotz dieser Unterschiede und globaler Signifikanz der Änderungen in den Phasenmittelwerten waren die hier getesteten Paarvergleiche der einzelnen Messphasen nicht signifikant (Tabelle 11, Seite 54).

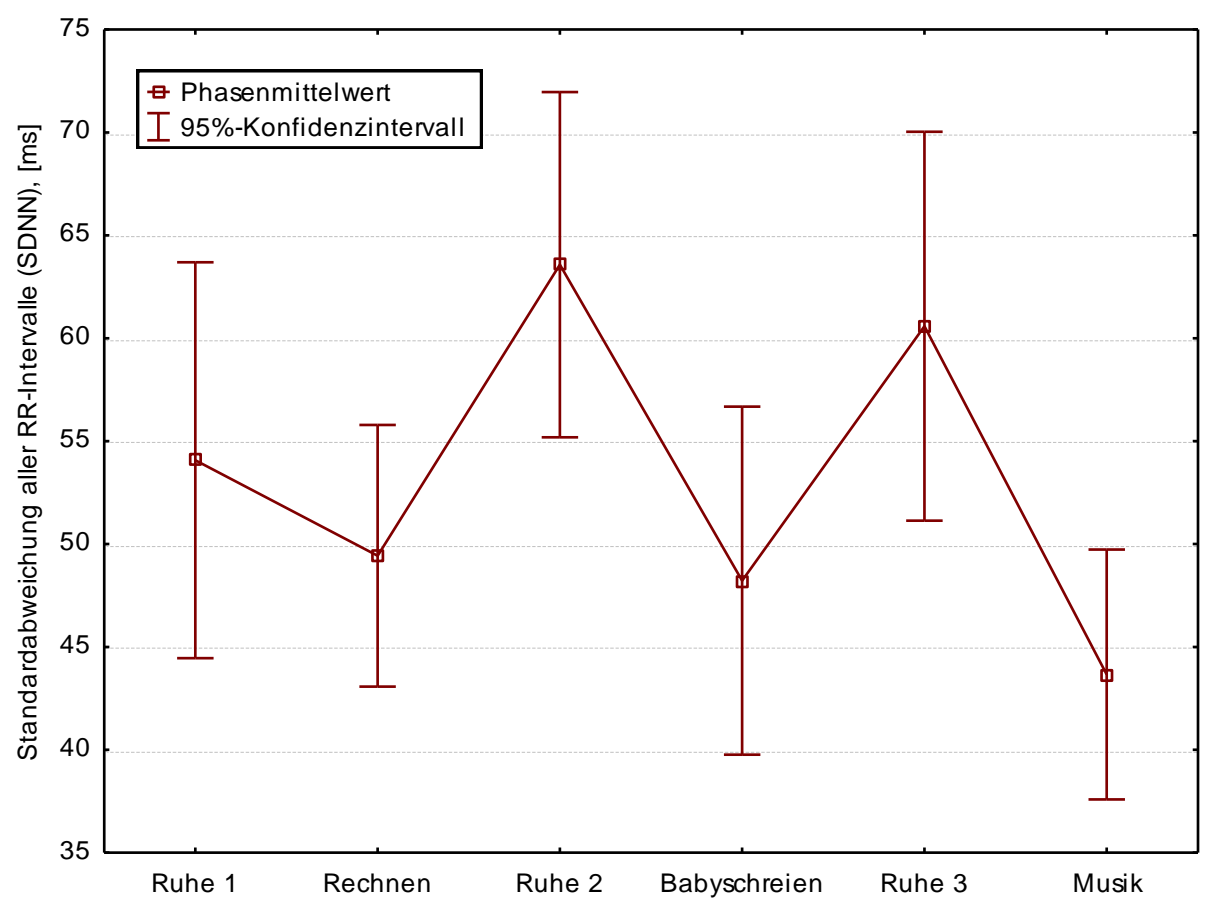

Abbildung 20:

Verlauf der Standardabweichung aller RR-Intervalle (SDNN) während der Stressbelastungsuntersuchung, Phasenmittelwerte und 95\%-Konfidenzintervalle

\subsubsection{Hochfrequenzband der Herzratenvariabilität (HF-HRV)}

Der Verlauf der Phasenmittelwerte für den Parasympathikus-Marker HF-HRV ist in Abbildung 21 (Seite 60) dargestellt. Phasenunterschiede konnten für die HF-HRV statistisch nicht bestätigt werden, bereits die globale ANOVA-Analyse war nicht signifikant. Betrachtet man jedoch den graphischen Verlauf der Phasenmittelwerte, so zeigt sich wie erwartet ein leichter Abfall der HF-HRV 
(logarithmierte Werte) während des Kopfrechentests von 4,84 $\mathrm{ms}^{2}$ (Ruhe 1) auf 4,74 $\mathrm{ms}^{2}$ sowie ein Anstieg während der Entspannungsmusik auf 4,91 ms². Während des Babyschreiens nimmt die HFHRV ähnliche Werte wie zu Beginn der Messung (Ruhe 1) an.

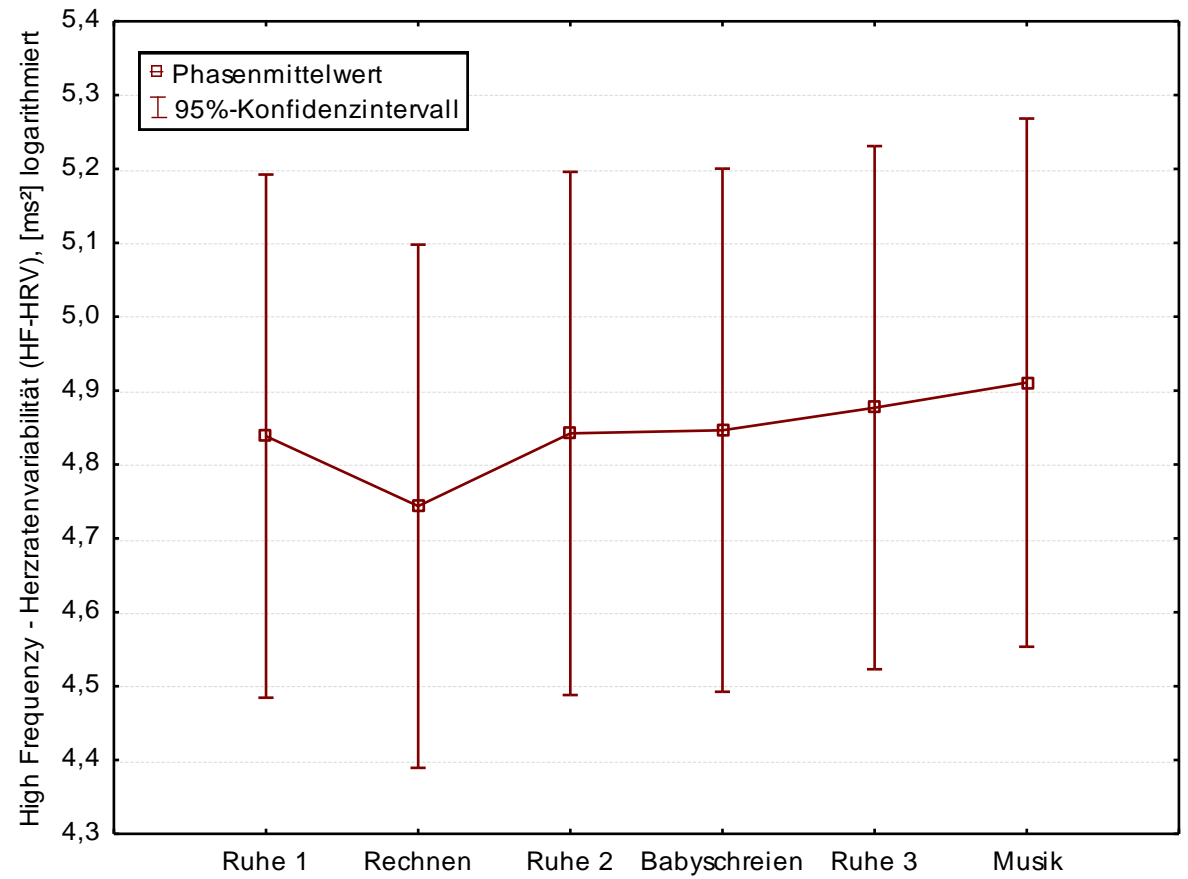

\section{Abbildung 21: Verlauf der HF-HRV (logarithmiert) während der Stressbelastungsuntersuchung, Phasenmittelwerte und 95\%-Konfidenzintervalle}

\subsubsection{Niederfrequenzband der Herzratenvariabilität (LF-HRV)}

Als weiterer autonomer Parameter, der vorwiegend sympathisch vermittelt ist, wurde die LF-HRV betrachtet (Abbildung 22, Seite 61), welche im Unterschied zur HF-HRV in der globalen Berechnung einen Zeiteffekt $(\mathrm{p}<0,001)$ und signifikante Phasenunterschiede zwischen Baseline und Rechentest sowie zwischen den beiden Stressoren aufweist (p-Wert jeweils <0,001; Tabelle 12, Seite 55).

Im Verlauf ist ein deutlicher Anstieg der logarithmierten Werte der LF-HRV von anfangs 5,29 ms ${ }^{2}$ auf $5,70 \mathrm{~ms}^{2}$ während des Rechnens festzustellen (Phasenmittelwert-Differenz 0,41 ms ${ }^{2}$ ), welcher auch in der anschließenden Ruhephase nicht absinkt. Während des Babyschreiens erreicht die LF-HRV unerwarteterweise ihren Minimalwert mit 5,22 $\mathrm{ms}^{2}$ und steigt danach wieder auf 5,69 $\mathrm{ms}^{2}$ an. Somit ergibt sich auch ein statistisch signifikanter Unterschied $(p<0,001)$ für die beiden Stressoren „Rechnen“ und „Babyschreien“ mit einer Phasenmittelwert-Differenz von 0,47 $\mathrm{ms}^{2}$. Nach den beiden Stresstests sinkt die LF-HRV nicht wie erwartet ab, sondern steigt sogar geringfügig an, sodass die Paarvergleiche zwischen der Ruhephase 1 und Ruhephase 2 (Phasenmittelwert-Differenz 0,49 $\mathrm{ms}^{2}$ ) bzw. Ruhephase 3 (Phasenmittelwert-Differenz 0,40 $\mathrm{ms}^{2}$ ) signifikant ausfallen (p-Wert jeweils $<0,001)$. Die entspannende Musik erzielte erwartungsgemäß ein Absinken der LF-HRV auf Ausgangsniveau (Ruhe 1). 


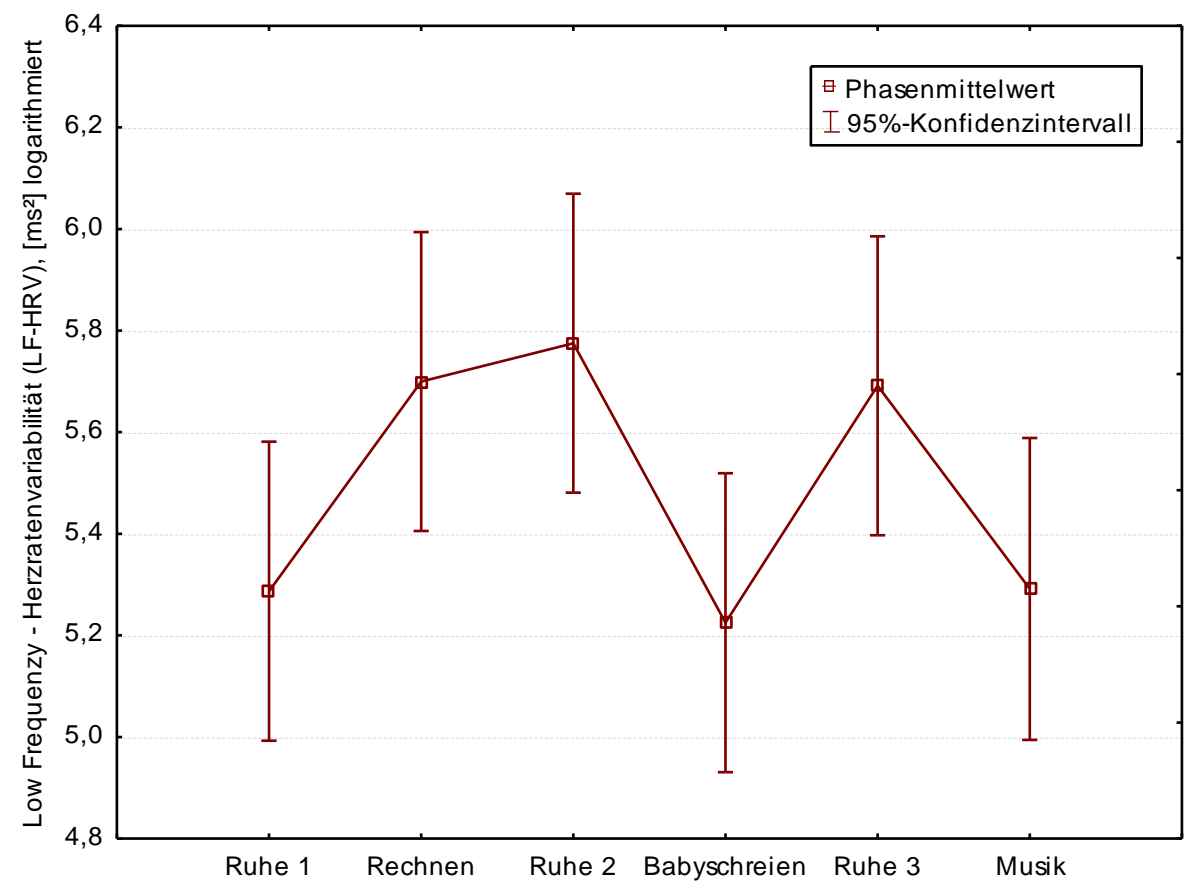

Abbildung 22: Verlauf der LF-HRV (logarithmiert) während der Stressbelastungsuntersuchung, Phasenmittelwerte und 95\%-Konfidenzintervalle

\subsubsection{Barorezeptorsensitivität (BRS)}

Der Verlauf der BRS (Slope Mean Total) ist in Abbildung 23 (Seite 61) dargestellt. Vergleichbare Ergebnisse stellten sich auch für die Up- und Down-Events der BRS heraus.

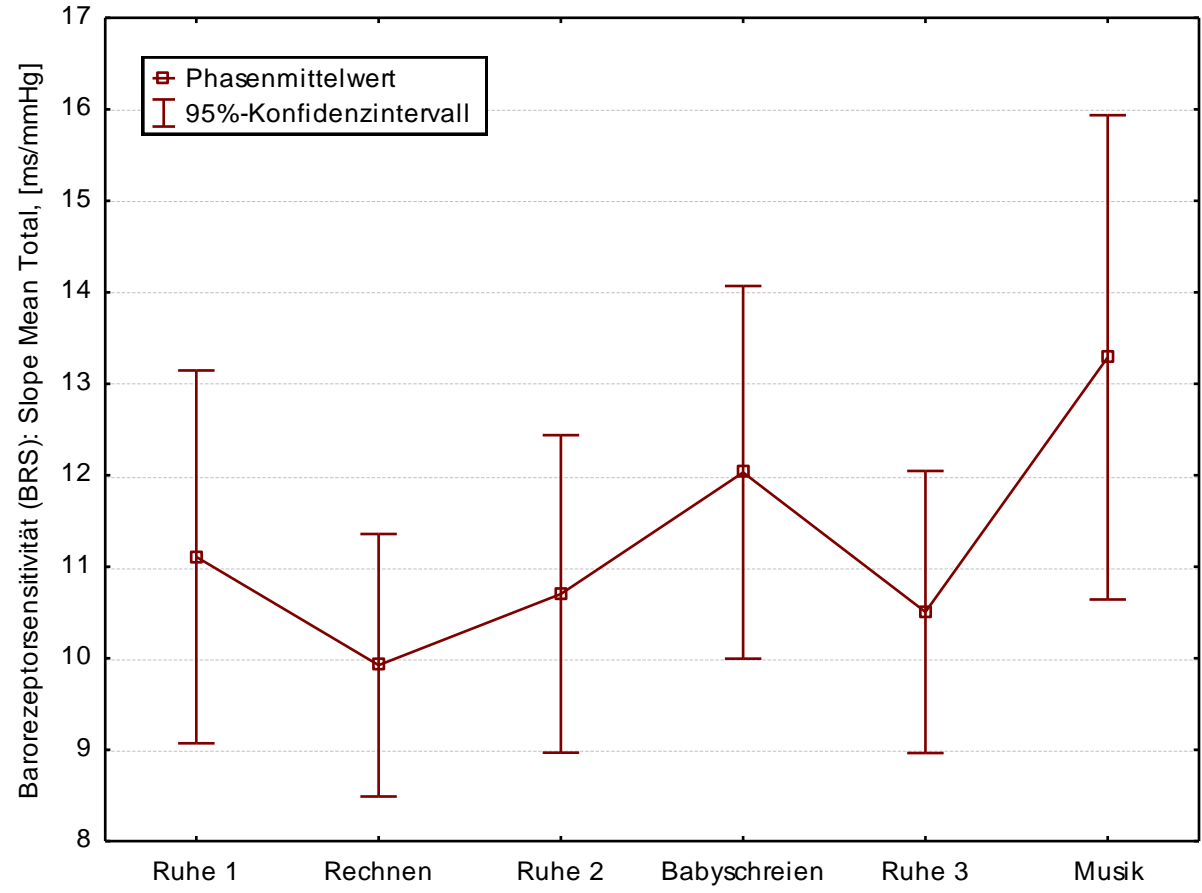

Abbildung 23: $\quad$ Verlauf der Barorezeptorsensitivität (Slope Mean Total) während der Stressbelastungsuntersuchung, Phasenmittelwerte und 95\%-Konfidenzintervalle 
Die explorative Auswertung der BRS offenbarte einen gegenläufigen Effekt der beiden Stressoren, der durch einen p-Wert <0,01 im Paarvergleich bestätigt wurde (Phasenmittelwert-Differenz 2,2 ms/mmHg; Tabelle 12, Seite 55). Während der Rechentest zu einer Abnahme der BRS von anfänglich $11,1 \mathrm{~ms} / \mathrm{mmHg}$ (Ruhe 1) auf 9,92 ms/mmHg führte, resultierte das Babyschreien in einer Zunahme der BRS auf 12,0 ms/mmHg. Darüber hinaus zeigte sich während der Entspannungsmusik eine weitere Steigerung der BRS auf den maximalen Phasenmittelwert von $13,3 \mathrm{~ms} / \mathrm{mmHg}$, die sich auch im Paarvergleich zwischen Baseline und Musik (Phasenmittelwert-Differenz 2,2 ms/mmHg) als signifikant erwies $(\mathrm{p}<0,01)$.

\subsubsection{Vergleich der beiden Stresstests „Rechnen“ und „Babyschreien“}

Die beiden spezifischen Stresstests „Kopfrechnen“ und „Babyschreien“ rufen bei den PTBSPatientinnen eine unterschiedliche autonome kardiovaskuläre Aktivierung hervor. Abbildung 24 (Seite 62) zeigt das Reaktionsverhalten der TFM-Parameter (Zunahme $\uparrow$, Abnahme $\downarrow$ bzw. kaum Veränderung $\leftrightarrow$ ) während der beiden Stresstests im Vergleich zu den Ausgangswerten in Ruhe 1 sowie ggf. Angaben des Signifikanzniveaus für die Paarvergleiche Ruhe 1 vs. Stressor bzw. Rechnen vs. Babyschreien.

Vergleich der Phasenmittelwerte: Ruhe 1 vs. Stresstests, Rechnen vs. Babyschreien

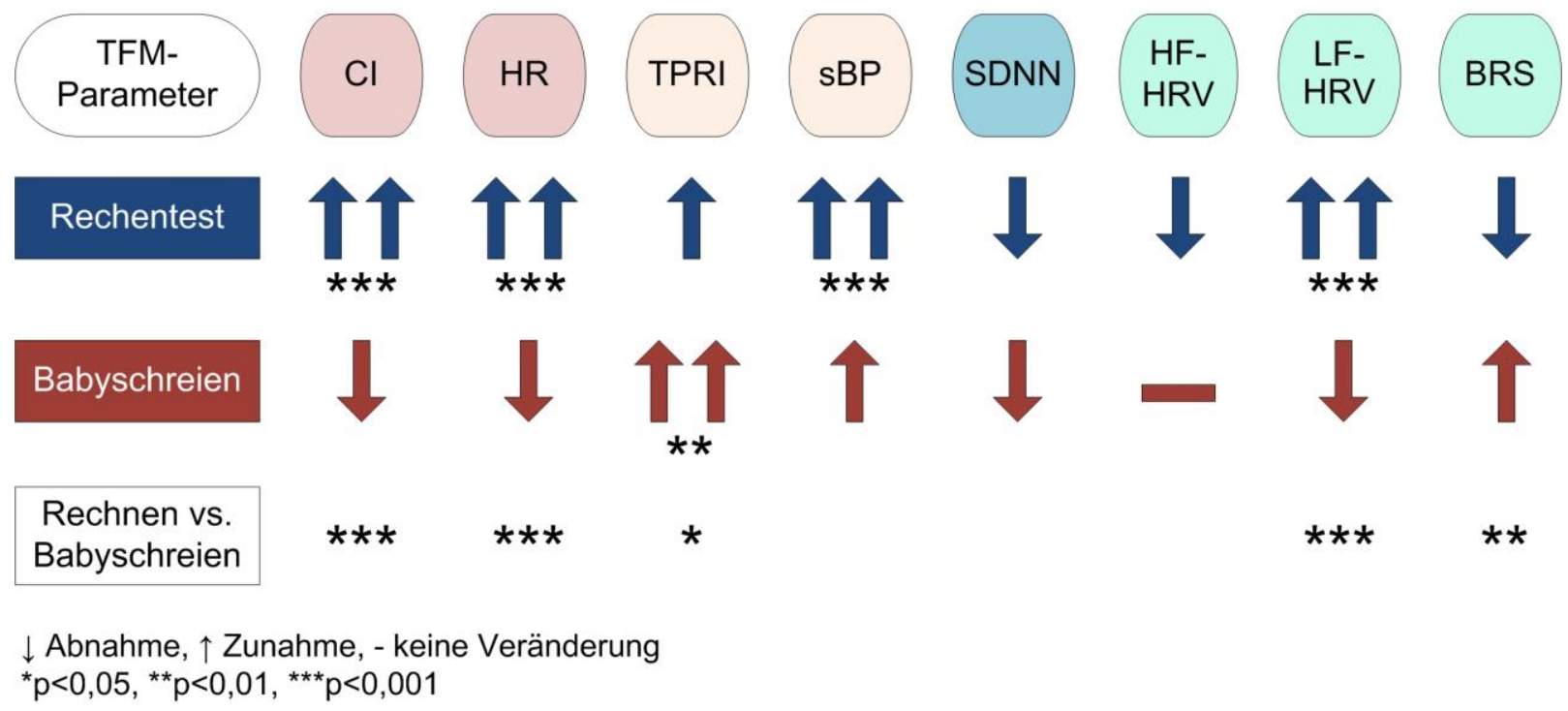

Abbildung 24: Verhalten der TFM-Parameter während der beiden Stresstests „Rechnen“ und „Babyschreien“ in Bezug zur Ruhephase 1 (Baseline), Signifikanzen des Phasenvergleiches Ruhe 1 vs. Stresstest sowie Rechnen vs. Babyschreien: *p<0,05, $* * \mathbf{p}<0,01, * * \mathbf{p}<\mathbf{0 , 0 0 1}$ (adjustiert)

Das Reaktionsmuster während des Rechentests fällt wie in den Hypothesen erwartet aus. Es zeigt sich im Vergleich zur Ruhephase 1 (Baseline) im Bereich der hämodynamischen Parameter eine Steigerung des CI $(p<0,001)$ sowie der HR $(p<0,001)$ und eine Zunahme TPRI (nicht signifikant) mit 
Anstieg des sBP $(\mathrm{p}<0,001)$. Auch die autonomen TFM-Parameter spiegeln eine typische Stressantwort mit deutlicher Zunahme der LF-HRV ( $p<0,001)$ sowie leichter Abnahme der HF-HRV, der SDNN und der BRS (jeweils insignifikanter Paarvergleich mit Ruhephase 1) wider.

Unter Babyschreien kommt es im Vergleich zum Kopfrechnen wie erwartet zu einer stärkeren Zunahme des TPRI, welche auch im Paarvergleich zwischen Ruhe 1 und Babyschreien sowie zwischen den beiden Stressoren signifikant ist ( $p$-Wert jeweils <0,05). Die einhergehende Blutdrucksteigerung während des Babyschreiens erreicht im Vergleich zum Rechnen keine Signifikanz. Der CI wird wie postuliert nicht nur geringer gesteigert sondern nimmt sogar im Vergleich zum Ausgangswert in Ruhe 1 weiter ab, hieraus ergibt sich ebenfalls ein signifikanter Unterschied zum Rechentest ( $\mathrm{p}<0,001$ ). Gleiches gilt für die HR. Der Phasenunterschied von Ruhe 1 und Babyschreien ist im Gegensatz zum Rechentest für den CI und die HR nicht signifikant. Die SDNN sinkt im Vergleich zum Rechentest stärker ab, der Unterschied ist jedoch nicht signifikant. Für die weiteren autonomen Parameter zeigt sich interessanterweise ein gegensätzliches Reaktionsverhalten während des Babyschreiens im Vergleich zum Rechentest. Die Phasenmittelwerte der BRS steigen an, während die LF-HRV sinkt. Für die LF-HRV ( $p<0,001)$ und die BRS $(p<0,01)$ ist dieser Unterschied zwischen den Stressoren statistisch signifikant. Die HF-HRV verändert sich kaum in Bezug zum Ausgangswert in Ruhe 1.

\subsubsection{Erholung nach Stressanforderung „Babyschreien“}

Um die Rückbildungsfähigkeit der Stressparameter nach der Stressanforderung „Babyschreien“ besser beurteilen zu können, wurden neben dem Vergleich der Phasenmittelwerte zusätzliche Messzeitpunkte betrachtet. Für das Babyschreien wurden fünf Herzschläge, beginnend 30 Sekunden vor Schluss ausgewertet, in der anschließenden Ruhephase wurden die Werte nach 30, 60 und 90 Sekunden bestimmt (siehe Methodenteil Kapitel 3.7.6, Seite 32).

Die Mittelwerte der fünf Herzschläge \pm Standardabweichung sowie die Ergebnisse der globalen ANOVA sind in Tabelle 13 (Seite 63) dargestellt.

Tabelle 13: Ausgewählte TFM-Parameter (CI, sBP, TPRI, RMSSD) zur Beurteilung der Erholung nach Babyschreien für vier Messzeitpunkte: Mittelwerte von jeweils fünf Herzschlägen \pm Standardabweichung und Anzahl [n], ggf. p-Wert der ANOVA

\begin{tabular}{|c|c|c|c|c|c|}
\hline \multirow{2}{*}{$\begin{array}{c}\text { TFM- } \\
\text { Parameter } \\
\text { (Erholung) }\end{array}$} & $\begin{array}{c}\text { Babyschreien } \\
(4: 30 \mathrm{~min})\end{array}$ & $\begin{array}{c}\text { Ruhe 3 } \\
(0: 30 \mathrm{~min})\end{array}$ & $\begin{array}{c}\text { Ruhe 3 } \\
\text { (1:00min) }\end{array}$ & $\begin{array}{c}\text { Ruhe 3 } \\
(1: 30) \\
\end{array}$ & \multirow{2}{*}{$\begin{array}{c}\text { p- } \\
\text { Wert }\end{array}$} \\
\hline & \multicolumn{4}{|c|}{ Mittelwert \pm Standardabweichung [n] } & \\
\hline$\underset{\left(1 / \mathrm{min}^{*} \mathrm{~m}^{2}\right)}{\mathbf{C I}}$ & $\begin{array}{c}2,79 \pm 0,78 \\
{[51]}\end{array}$ & $\begin{array}{c}3,03 \pm 0,95 \\
{[51]}\end{array}$ & $\begin{array}{c}2,89 \pm 0,77 \\
{[51]}\end{array}$ & $\begin{array}{c}2,88 \pm 0,71 \\
{[51]}\end{array}$ & n.s. \\
\hline $\begin{array}{c}\mathbf{S B P} \\
(\mathrm{mmHg})\end{array}$ & $\begin{array}{c}118,76 \pm 18,06 \\
{[52]}\end{array}$ & $\begin{array}{c}119,84 \pm 20,00 \\
{[52]}\end{array}$ & $\begin{array}{c}120,04 \pm 18,19 \\
{[52]}\end{array}$ & $\begin{array}{c}119,18 \pm 17,86 \\
{[52]}\end{array}$ & n.s. \\
\hline $\begin{array}{c}\text { TPRI } \\
\left(\text { dyn }^{2}{ }^{2} \mathrm{~m}^{2} / \mathrm{cm}^{5}\right)\end{array}$ & $\begin{array}{c}2685,33 \pm \\
941,25 \\
{[52]}\end{array}$ & $\begin{array}{c}2503,33 \\
\pm 887,63 \\
{[52]}\end{array}$ & $\begin{array}{c}2569,02 \pm \\
795,52 \\
{[52]}\end{array}$ & $\begin{array}{c}2544,19 \pm \\
710,31 \\
{[52]}\end{array}$ & n.s. \\
\hline $\begin{array}{c}\log \text { RMSSD } \\
(\mathrm{ms})\end{array}$ & $\begin{array}{c}3,80 \pm 0,89 \\
{[52]}\end{array}$ & $\begin{array}{c}3,76 \pm 0,90 \\
{[52]}\end{array}$ & $\begin{array}{c}3,69 \pm 0,85 \\
{[52]}\end{array}$ & $\begin{array}{c}3,64 \pm 0,76 \\
{[52]}\end{array}$ & n.s. \\
\hline
\end{tabular}


In diese besondere Auswertung der Erholungsphase wurden $\mathrm{zu}$ den oben angegebenen Messzeitpunkten jeweils fünf Schläge der folgenden vier Parameter eingeschlossen: der Cardiac Index (CI), der systolische Blutdruck (sBP), der totale periphere Gefäßwiderstands-Index (TPRI) und die Root Mean Square of Successive Differences (RMSSD).

Für keinen der vier TFM-Parameter fand sich eine signifikante kurzfristige Änderung in der Erholungsphase nach dem Babyschreien.

\subsection{Patientinnen-Subgruppe ,hoch Borderline“}

Der Patientinnen-Subgruppe "hoch Borderline" wurden nach den zuvor festgelegten Kriterien (BPI Cut-20 $\geq 10$, BSL-Subskala Affektregulation >25, BSL-Subskala Autoaggression >17,5) 16 Patientinnen zugeordnet. Die Vergleichsgruppe der restlichen PTBS-Patientinnen ohne ausgeprägte Borderline-Merkmale zählt 30 Teilnehmerinnen (siehe Methodenteil Kapitel 3.8, Seite 34).

Die Subgruppe wurde der Vollständigkeit halber zum Vergleich der allgemeinen soziodemographischen Daten der Vergleichsgruppe gegenübergestellt. Die t-Tests für unverbundene Stichproben bzw. der U-Test nach Mann-Whitney erbrachten keinen signifikanten Unterschied zwischen Subgruppe und Vergleichsgruppe für die Merkmale Alter (Subgruppe: 41,38 $\pm 7,36$, Kontrolle: 41,63 $\pm 8,50$ ), Familienstand, Partnerschaft, Schulabschluss, Ausbildung und Berufstätigkeit. Auch die Anzahl problematischer Medikamente (herzwirksam bzw. anticholinerg) unterschied sich nicht signifikant. Die Ergebnisse der Gegenüberstellung der psychometrischen Befunde sind tabellarisch dargestellt (Tabelle 14, Seite 65).

\subsubsection{Psychometrischer Befund der Subgruppe „hoch Borderline“}

Für den Cut-20 des BPI zeigten sich hochsignifikante Unterschiede $(\mathrm{p}<0,001)$ zwischen Subgruppe „hoch Borderline“ und Vergleichskollektiv. Ab einem Cut-20-Wert $\geq 10$ kann eine BorderlinePersönlichkeitsstörung angenommen werden. Diesen haben die Patientinnen der Subgruppe mit einem Durchschnitt von 14,25 weit überschritten und waren somit eindeutig vom Rest der untersuchten Patientinnen, die nur einen Mittelwert des Cut-20 von 9,37 erreichten, abzugrenzen. Neben dem Cut20 sind auch fast alle Subskalen des BPI signifikant unterschiedlich. Für die Subskalen Identitätsdiffusion und primitive Abwehr ergab sich ein p-Wert $<0,01$, für die Subskala Angst vor Nähe sogar ein $\mathrm{p}$-Wert $<0,001$. Lediglich die Subskala mangelhafte Realitätsprüfung war nicht signifikant unterschiedlich.

Auch für die Subskalen Affektregulation sowie Autoaggression der BSL, welche als zusätzliche Kriterien für die Subgruppenbildung festgelegt wurden, ergab sich eine deutliche Signifikanz (p-Wert jeweils <0,001). Darüber hinaus waren weitere Subskalen dieses Fragebogens im Vergleich von Subgruppe und Vergleichsgruppe signifikant unterschiedlich, nämlich die Subskalen Selbstwahrnehmung, soziale Isolation und Intrusionen sowie die Gesamtskala mit jeweils einem p- 
Wert <0,001. Lediglich die Subskala Dysphorie zeigte keinen signifikanten Unterschied. Für die

Subskala Feindseligkeit konnte eine Tendenz $(\mathrm{p}=0,067)$ festgestellt werden.

Tabelle 14: Psychometrischer Befund der Subgruppe "hoch Borderline" und Vergleichsgruppe

\begin{tabular}{|c|c|c|c|c|c|c|c|}
\hline \multirow{2}{*}{ Psychometrische Skalen } & \multicolumn{3}{|c|}{$\begin{array}{c}\text { Subgruppe } \\
\text {,hoch Borderline } 6\end{array}$} & \multicolumn{3}{|c|}{ Vergleichsgruppe } & \multirow[t]{2}{*}{ p-Wert } \\
\hline & $\mathrm{n}$ & $\begin{array}{c}\text { Mittel- } \\
\text { wert }\end{array}$ & $\mathrm{SD}$ & $\mathrm{n}$ & $\begin{array}{c}\text { Mittel- } \\
\text { wert }\end{array}$ & SD & \\
\hline \multicolumn{8}{|l|}{ SCL-90-R } \\
\hline Somatisierung & 16 & 1,62 & 0,78 & 29 & 1,23 & 0,83 & n.s. \\
\hline Zwanghaftigkeit & 16 & 2,33 & 0,77 & 29 & 1,68 & 0,91 & $<0,05$ \\
\hline Unsicherheit im Sozialkontakt & 16 & 2,74 & 0,85 & 29 & 1,50 & 0,84 & $<0,001$ \\
\hline Depressivität & 16 & 2,74 & 0,59 & 29 & 1,99 & 1,10 & $<0,01$ \\
\hline Ängstlichkeit & 16 & 2,34 & 0,79 & 29 & 1,63 & 0,92 & $<0.05$ \\
\hline Aggressivität & 16 & 1,10 & 0,53 & 29 & 0,94 & 0,86 & n.s. \\
\hline phobische Angst & 16 & 2,03 & 0,90 & 29 & 1,18 & 0,90 & $<0,01$ \\
\hline paranoides Denken & 16 & 1,70 & 0,84 & 29 & 1,04 & 0,75 & $<0,05$ \\
\hline Psychotizismus & 16 & 1,70 & 0,62 & 29 & 0,91 & 0,72 & $<0,001$ \\
\hline Global Severity Index & 16 & 2,12 & 0,48 & 29 & 1,42 & 0,66 & $<0,001$ \\
\hline \multicolumn{8}{|l|}{ FDS } \\
\hline FDS-Gesamtwert & 16 & 26,24 & 15,33 & 30 & 14,68 & 10,52 & $<0,05$ \\
\hline DES-Gesamtwert & 16 & 30,69 & 18,18 & 30 & 16,69 & 11,95 & $<0,05$ \\
\hline DES-T & 16 & 23,05 & 19,83 & 30 & 12,92 & 11,62 & n.s. \\
\hline Dissoziative Amnesie & 16 & 13,98 & 12,90 & 30 & 8,00 & 10,33 & n.s. \\
\hline $\begin{array}{l}\text { Tendenz zu Imaginativen } \\
\text { Erlebnisweisen }\end{array}$ & 16 & 38,96 & 19,26 & 30 & 22,80 & 14,23 & $<0,01$ \\
\hline Depersonalisation/Derealisation & 16 & 29,69 & 23,79 & 30 & 14,28 & 13,92 & $<0,05$ \\
\hline $\begin{array}{l}\text { pseudoneurologische } \\
\text { Konversionssymptome }\end{array}$ & 16 & 17,89 & 13,56 & 30 & 11,28 & 11,32 & n.s. \\
\hline ICD-10 Symptome & 16 & 18,42 & 11,52 & 30 & 11,17 & 9,80 & $<0,05$ \\
\hline \multicolumn{8}{|l|}{ IES-R } \\
\hline Intrusion & 16 & 25,69 & 6,03 & 26 & 24,12 & 8,68 & n.s. \\
\hline Vermeidung & 16 & 28,69 & 6,72 & 26 & 24,85 & 9,83 & n.s. \\
\hline Übererregung & 16 & 25,50 & 7,12 & 26 & 22,35 & 8,01 & n.s. \\
\hline \multicolumn{8}{|l|}{ BDI } \\
\hline Gesamtwert & 16 & 34,69 & 9,24 & 29 & 25,72 & 10,66 & $<0,01$ \\
\hline \multicolumn{8}{|l|}{ BPI } \\
\hline Identitätsdiffusion & 16 & 7,94 & 2,74 & 30 & 5,13 & 3,38 & $<0,01$ \\
\hline Angst vor Nähe & 16 & 5,69 & 1,30 & 30 & 3,80 & 1,92 & $<0,001$ \\
\hline Primitive Abwehr & 16 & 5,94 & 2,32 & 30 & 3,23 & 1,98 & $<0,01$ \\
\hline mangelhafte Realitätsprüfung & 16 & 1,13 & 1,41 & 30 & 0,70 & 1,18 & n.s. \\
\hline Cut-20 & 16 & 14,25 & 3,22 & 30 & 9,37 & 3,96 & $<0,001$ \\
\hline \multicolumn{8}{|l|}{ BSL } \\
\hline Selbstwahrnehmung & 16 & 36,56 & 13,46 & 30 & 19,83 & 12,95 & $<0,001$ \\
\hline Affektregulation & 16 & 36,50 & 6,42 & 30 & 20,07 & 9,95 & $<0,001$ \\
\hline Autoaggression & 16 & 28,00 & 7,69 & 30 & 11,87 & 8,78 & $<0,001$ \\
\hline Dysphorie & 16 & 33,50 & 5,03 & 30 & 31,33 & 5,05 & n.s. \\
\hline soziale Isolation & 16 & 22,44 & 10,28 & 30 & 9,60 & 7,97 & $<0,001$ \\
\hline Intrusionen & 16 & 17,06 & 6,77 & 30 & 8,40 & 7,38 & $<0,001$ \\
\hline Feindseligkeit & 16 & 7,25 & 4,31 & 30 & 4,80 & 3,83 & 0,067 \\
\hline Gesamtskala & 16 & 214,06 & 41,18 & 30 & 123,47 & 51,51 & $<0,001$ \\
\hline
\end{tabular}


Zusammenfassend zeigen die beiden Borderline-spezifischen Messinstrumente BPI und BSL auch auf den nicht für die Gruppeneinteilung benutzten Subskalen erwartungsgemäß deutliche Unterschiede zwischen der Subgruppe „hoch Borderline“ und der Vergleichsgruppe auf.

Da die BPS ein komplexes Symptomspektrum umfasst, zeichneten sich auch für andere Fragebögen Signifikanzen für die Patientinnen-Untergruppe mit starker Borderline-Symptomatik im Vergleich zum restlichen Patientinnenkollektiv ab. Die Patientinnen der Subgruppe „hoch Borderline“ waren laut BDI stärker depressiv $(\mathrm{p}<0,01)$ und zeigten auch erhöhte Gesamtwerte im FDS und DES ( $\mathrm{p}$-Wert jeweils <0,05). Die genauen Unterschiede für die SCL-90-R sind Tabelle 14 (Seite 65) zu entnehmen.

\subsubsection{Subjektives Stressempfinden und vegetative bzw. emotionale Reaktionen}

Die Angaben des subjektiven Stressempfindens während der TFM-Messung erbrachten keine signifikanten Unterschiede zwischen der Subgruppe „hoch Borderline“ und der Kontrollgruppe (Tabelle 15, Seite 66).

Auch die vor Beginn der Untersuchung gestellte Frage, ob sich die Patientinnen zurzeit besonders gestresst fühlten, beantworteten die Gruppen gleichermaßen.

Tabelle 15: Angaben des subjektiven Stressempfindens während der Stressbelastungsuntersuchung von Subgruppe "hoch Borderline" [n=16] und Vergleichsgruppe [n=30], Mittelwerte \pm Standardabweichung und ggf. p-Wert des t-Tests

\begin{tabular}{|c|c|c|c|c|}
\hline $\begin{array}{c}\text { Subgruppenvergleich } \\
\text { des Subjektiven } \\
\text { Stressempfindens }\end{array}$ & $\begin{array}{c}\text { Ruhe 1 } \\
\text { (Baseline) }\end{array}$ & Rechentest & Babyschreien & $\begin{array}{c}\text { Entspannungs- } \\
\text { musik }\end{array}$ \\
\cline { 2 - 5 } & \multicolumn{3}{|c|}{ Mittelwert \pm Standardabweichung, ggf. p-Wert } \\
\hline $\begin{array}{c}\text { Subgruppe } \\
\text { „hoch Borderline“ [16] }\end{array}$ & $3,90 \pm 1,89$ & $6,22 \pm 1,94$ & $6,28 \pm 2,85$ & $5,13 \pm 3,16$ \\
\hline Vergleichsgruppe [30] & $3,17 \pm 1,86$ & $6,55 \pm 1,77$ & $6,32 \pm 2,28$ & $4,27 \pm 2,78$ \\
\hline ggf. p-Wert & n.s. & n.s. & n.s. & n.s. \\
\hline
\end{tabular}

Für die während der TFM-Messung beobachteten vegetativen bzw. emotionalen Reaktionen (schweres Atmen, Erregungszustände, Weinen bzw. Tränen in den Augen, Nutzen von Copingmechanismen, Numbing) im Einzelnen fand sich im $\mathrm{Chi}^{2}$-Test kein signifikanter Unterschied zwischen den hoch Borderline-Patientinnen und den restlichen Frauen während der beiden Stresstests und während der Entspannungsmusik.

Prozentual gesehen zeigen die Patientinnen der Subgruppe „hoch Borderline“ jedoch etwas häufiger eine schwere Atmung, Weinen bzw. Tränen in den Augen sowie ein Numbing-Verhalten während des Babyschreiens. Die hoch Borderline-Patientinnen hatten zudem häufiger zwei oder mehrere Reaktionsformen gleichzeitig (43,8\%) im Unterschied zu den restlichen Patientinnen (20,0\%), was jedoch im Gruppenvergleich ebenfalls kein signifikant unterschiedliches Ergebnis ergab (Tabelle 16, Seite 67). 
Tabelle 16: Anzahl beobachteter vegetativer Reaktionen während des Babyschreiens von Subgruppe "hoch Borderline" [n=16] und Vergleichsgruppe [n=30], Häufigkeiten und Prozente

\begin{tabular}{|c|c|c|c|c|c|c|c|}
\hline \multirow{3}{*}{$\begin{array}{l}\text { Beobachtete } \\
\text { vegetative } \\
\text { Reaktionen } \\
\text { während } \\
\text { Babyschreien }\end{array}$} & \multirow[b]{2}{*}{$\begin{array}{l}\text { Schwere } \\
\text { Atmung }\end{array}$} & \multirow[b]{2}{*}{$\begin{array}{l}\text { Erre- } \\
\text { gung }\end{array}$} & \multirow[b]{2}{*}{ Weinen } & \multirow{2}{*}{$\begin{array}{l}\text { Coping- } \\
\text { mecha- } \\
\text { nismen }\end{array}$} & \multirow[b]{2}{*}{$\begin{array}{l}\text { Num- } \\
\text { bing }\end{array}$} & \multicolumn{2}{|c|}{ Gesamt } \\
\hline & & & & & & $\leq 1$ & $>1$ \\
\hline & \multicolumn{7}{|c|}{ Häufigkeiten (Prozente) } \\
\hline $\begin{array}{l}\text { Subgruppe „hoch } \\
\text { Borderline“6 [16] }^{\text {(6) }}\end{array}$ & $\begin{array}{c}6 \\
(37,5 \%)\end{array}$ & $\begin{array}{c}3 \\
(18,8 \%)\end{array}$ & $\begin{array}{c}2 \\
(12,5 \%)\end{array}$ & $\begin{array}{c}3 \\
(18,8 \%)\end{array}$ & $\begin{array}{c}3 \\
(18,8 \%)\end{array}$ & $\begin{array}{c}9 \\
(56,2 \%)\end{array}$ & $\begin{array}{c}7 \\
(43,8 \%)\end{array}$ \\
\hline $\begin{array}{c}\text { Vergleichsgruppe } \\
{[30]}\end{array}$ & $\begin{array}{c}8 \\
(26,7 \%)\end{array}$ & $\begin{array}{c}8 \\
(26,7 \%)\end{array}$ & $\begin{array}{c}2 \\
(6,7 \%)\end{array}$ & $\begin{array}{c}6 \\
(20,0 \%)\end{array}$ & $\begin{array}{c}2 \\
(6,7 \%)\end{array}$ & $\begin{array}{c}24 \\
(80,0 \%)\end{array}$ & $\begin{array}{c}6 \\
(20,0 \%)\end{array}$ \\
\hline p-Wert $\left(\mathrm{Chi}^{2}\right.$-Test $)$ & n.s. & n.s. & n.s. & n.s. & n.s. & \multicolumn{2}{|c|}{ n.s., $p=0,088$} \\
\hline
\end{tabular}

Eine Korrelationsanalyse zum Zusammenhang von beobachteten vegetativen Reaktionen (Gesamtanzahl) und physiologischen Parametern während des Babyschreiens, zeigte in beiden Gruppen signifikante Korrelationen für die HR, die LF-HRV sowie die BRS (Tabelle 17, Seite 67). Die HR korrelierte in der Subgruppe „hoch Borderline“ um 0,58 mit den vegetativen Reaktionen, bei den restlichen Patientinnen um 0,34. Für die HF-HRV fand sich eine negative Korrelation von -0,48 in der Subgruppe und -0,38 in der Vergleichsgruppe. Die LF-HRV hingegen zeigte nur in der Subgruppe eine negative Korrelation von $-0,45$. Die negative Korrelation der BRS zeigt mit $-0,47$ in der Subgruppe und -0,46 in der Kontrollgruppe ähnliche Werte in beiden Kollektiven.

Tabelle 17: Korrelation nach Kendall-Tau-b zwischen den beobachteten vegetativen Reaktionen und den TFM-Parametern während des Babyschreiens für die Subgruppe "hoch Borderline" $[n=16]$ und die Vergleichsgruppe $[n=30]$, Korrelationskoeffizient und ggf. Signifikanzniveau ( $\left.{ }^{*} \mathbf{p}<0,05, * * \mathbf{p}<0,01\right)$

\begin{tabular}{|c|c|c|c|c|c|c|c|c|}
\hline \multicolumn{7}{|c|}{$\begin{array}{c}\text { Korrelation der Anzahl beobachteter vegetativer Reaktionen } \\
\text { mit TFM-Parametern während des Babyschreiens }\end{array}$} \\
\hline $\begin{array}{c}\text { Korrelation } \\
\text { nach } \\
\text { Kendall-Tau-b }\end{array}$ & HR & CI & TRPI & sBP & SDNN & $\begin{array}{c}\text { log HF- } \\
\text { HRV }\end{array}$ & $\begin{array}{c}\text { log LF- } \\
\text { HRV }\end{array}$ & $\begin{array}{c}\text { BRS } \\
\text { total }\end{array}$ \\
\cline { 2 - 9 } & \multicolumn{7}{|c|}{ Korrelationskoeffizient } & \\
$\begin{array}{c}\text { Subgruppe } \\
\text {,hoch } \\
\text { Borderline“ } \\
{[16]}\end{array}$ & $0,578^{* *}$ & $-0,112$ & 0,193 & 0,294 & $-0,294$ & $-0,477^{*}$ & $-0,456^{*}$ & $-0,477^{*}$ \\
\hline $\begin{array}{c}\text { Vergleichs- } \\
\text { gruppe } \\
{[30]}\end{array}$ & $0,336^{*}$ & 0,046 & $-0,028$ & $-0,034$ & $-0,034$ & $-0,381^{* *}$ & $-0,171$ & $-0,461^{* *}$ \\
\hline
\end{tabular}




\subsubsection{Reagibilitätsmuster während der Stressbelastungsuntersuchung}

Zunächst wurde eine globale zweifaktorielle repeated measures ANOVA für die verschiedenen physiologischen Parameter durchgeführt, in der die sechs Messphasen und die zwei Gruppen zusammen und auf ihre Wechselwirkung hin untersucht wurden. Neben dem Reagibilitätsmuster der Subgruppe „hoch Borderline“ wurde dabei auf globale Gruppeneffekte zwischen den Gruppen geachtet. Ein Paarvergleich der einzelnen Messphasen erfolgte nur bei Vorliegen einer globalen Wechselwirkung.

In der Subgruppe „hoch Borderline“ waren die drei Blutdruckmesswerte sBP, dBP und mBP signifikant unterschiedlich in der Gegenüberstellung mit der Vergleichsgruppe (p-Wert jeweils $<0,05$ ), wobei die Patientinnen mit ausgeprägter Borderline-Symptomatik durchgehend einen signifikant höheren Blutdruck aufwiesen.

Des Weiteren fand sich in der zweifaktoriellen ANOVA in der Subgruppe eine fast signifikant niedrigere totale BRS ( $\mathrm{p}=0,051)$ sowie eine signifikante Wechselwirkung für die BRS $(\mathrm{p}<0,05)$.

Die Parameter sBP und BRS, für die ein signifikanter bzw. annähernd signifikanter Gruppeneffekt gefunden werden konnte, werden in den folgenden Abschnitten graphisch dargestellt und ausführlich beschrieben (Abbildung 26, Seite 70 und Abbildung 27, Seite 71).

Die Reaktivität der restlichen hämodynamischen und autonomen Parameter zeigte keinen signifikanten globalen Gruppenunterschied zwischen Subgruppe und Kontrollkollektiv, sodass auf eine graphische Darstellung und ausführliche Beschreibung an dieser Stelle verzichtet wird.

Des Weiteren wurde für einen besseren Vergleich der beiden Stressoren zwischen den Gruppen ein Paarvergleich Rechnen vs. Babyschreien für alle TFM-Parameter durchgeführt und die Phasenmittelwert-Differenzen der beiden Gruppen mittels t-Test verglichen (Tabelle 18, Seite 69). Hierbei zeigt sich ein signifikanter Unterschied der Änderungen des sBP und des TPRI (p-Wert jeweils $<0,05)$ sowie eine fast signifikant unterschiedliche Änderung der BRS total $(p=0,051)$ zwischen den Gruppen.

Abbildung 25 (Seite 69) veranschaulicht das Reaktionsverhalten (Zunahme $\uparrow$, Abnahme $\downarrow$ bzw. kaum Veränderung $\leftrightarrow$ ) der TFM-Parameter für den Phasenvergleich der beiden Stresstests „Rechnen“ und „Babyschreien“ und zeigt signifikant unterschiedliche Phasenmittelwert-Differenzen zwischen Subgruppe „hoch Borderline“ und Vergleichskollektiv. 
Tabelle 18: $\quad$ Paarvergleich der Stressoren (Rechnen vs. Babyschreien) für die TFM-Parameter HR, CI, TPRI, sBP, HF-HRV, LF-HRV, BRS in der Subgruppe "hoch Borderline" [n=16] und der Vergleichsgruppe $[n=30]$, Phasenmittelwert-Differenzen \pm Standardfehler sowie ggf. p-Wert

\begin{tabular}{|c|c|c|c|}
\hline \multirow{3}{*}{$\begin{array}{c}\text { Task-Force- } \\
\text { Monitor- } \\
\text { Parameter }\end{array}$} & Rechnen v & byschreien & \multirow{3}{*}{ ggf. p-Wert } \\
\hline & \multicolumn{2}{|c|}{ Phasenmittelwert -Differenzen \pm Standardfehler } & \\
\hline & $\begin{array}{c}\text { Subgruppe } \\
\text { „hoch Borderline“6 [16] }\end{array}$ & Vergleichsgruppe [30] & \\
\hline \multicolumn{4}{|c|}{ Hämodynamisch: Beta-adrenerg } \\
\hline $\begin{array}{c}\mathbf{C I} \\
\left(1 / \mathrm{min}^{*} \mathrm{~m}^{2}\right)\end{array}$ & $-0,29 \pm 0,08$ & $-0,24 \pm 0,08$ [29] & n.s. \\
\hline $\begin{array}{c}\text { HR } \\
\left(\min ^{-1}\right)\end{array}$ & $-3,34 \pm 1,91$ & $-4,35 \pm 0,74$ & n.s. \\
\hline \multicolumn{4}{|c|}{ Hämodynamisch: Alpha-adrenerg } \\
\hline $\begin{array}{c}\text { TPRI } \\
\left(\text { dyn }^{*} \mathrm{~s}^{*} \mathrm{~m}^{2} / \mathrm{cm}^{5}\right)\end{array}$ & $344,62 \pm 128,09$ & $51,31 \pm 65,66$ & $<0,05$ \\
\hline $\begin{array}{c}\mathbf{S B P} \\
(\mathrm{mmHg})\end{array}$ & $1,45 \pm 3,80$ & $-6,52 \pm 2,00$ & $<0,05$ \\
\hline \multicolumn{4}{|l|}{ Autonom } \\
\hline $\begin{array}{l}\text { SDNN } \\
(\mathrm{ms})\end{array}$ & $-1,92 \pm 7,25$ & $0,71 \pm 5,44$ & n.s. \\
\hline$\underset{\left(\mathrm{ms}^{2}\right)}{\log \mathbf{H F}-\mathbf{H R V}}$ & $-0,08 \pm 0,17$ & $0,24 \pm 0,10$ & n.s. \\
\hline $\begin{array}{c}\log \mathbf{L F}-\mathbf{H R V} \\
\left(\mathrm{ms}^{2}\right)\end{array}$ & $-0,52 \pm 0,18$ & $-0,42 \pm 0,14$ & n.s. \\
\hline $\begin{array}{c}\text { BRS total } \\
(\mathrm{ms} / \mathrm{mmHg})\end{array}$ & $0,39 \pm 0,62$ & $2,84 \pm 0,82$ & 0,051 \\
\hline
\end{tabular}

Paarvergleich Rechnen vs. Babyschreien

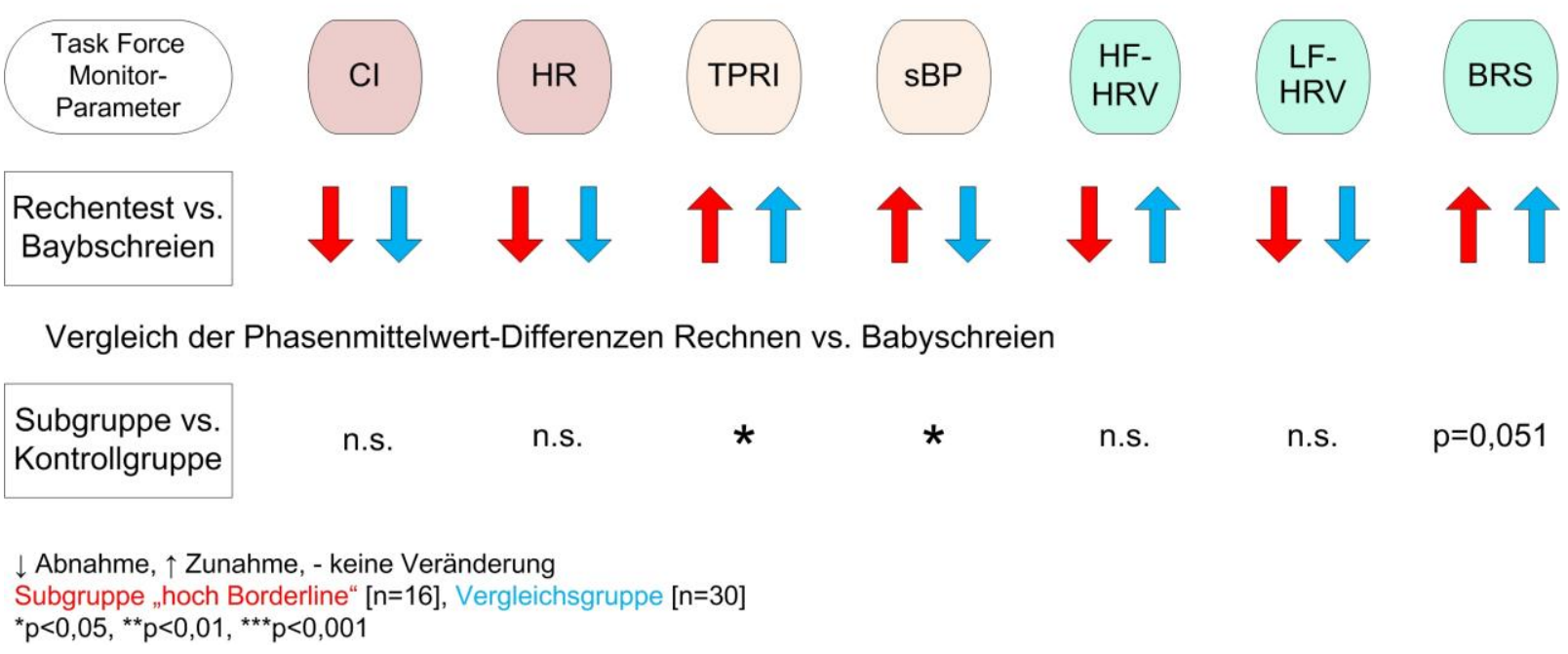

Abbildung 25: Paarvergleich Rechnen vs. Babyschreien: Verhalten der TFM-Parameter in der Subgruppe ,hoch Borderline“ [n=16] und in der Vergleichsgruppe [n=30], Signifikanzen des Gruppenvergleiches der Phasenmittelwert-Differenzen $\quad * \mathbf{p}<0,05, \quad * * \mathbf{p}<0,01$, $* * * \mathbf{p}<0,001$ 


\subsubsection{Systolischer arterieller Blutdruck (sBP)}

Die Patientinnen der Subgruppe „hoch Borderline“ weisen insgesamt deutlich höhere Blutdruckwerte als die Vergleichsgruppe auf. Es konnte ein signifikanter globaler Gruppeneffekt gefunden werden $(\mathrm{p}<0,05)$. Stellvertretend für alle Blutdruckmesswerte soll hier der sBP in seinem Verlauf während der Messphasen für die Subgruppe „hoch Borderline“ im Vergleich zur Kontrollgruppe dargestellt werden (Abbildung 26, Seite 70).

Der Ausgangsmittelwert des sBP der Subgruppe „hoch Borderline“ liegt bei 119,1 mmHg, in der Vergleichsgruppe beträgt dieser 112,0 mmHg. Während des Rechentests steigen die Blutdruckwerte in beiden Gruppen an. Während des Babyschreiens zeigt sich bei den hoch Borderline-Patientinnen ein deutlicher Anstieg auf den maximalen Phasenmittelwert von 126,5 mmHg. In der Vergleichsgruppe hingegen ist ein weiteres Absinken des Blutdruckes auf 114,3 mmHg während des Babyschreiens zu beobachten. Das unterschiedliche Verhalten des sBP in den beiden Gruppen spiegelt sich auch in einem Paarvergleich der beiden Stressoren wider, welcher in der Subgruppe eine PhasenmittelwertDifferenz von 1,5 mmHg und in der Vergleichsgruppe von -6,5 mmHg ergibt. Die Änderungen zwischen Rechnen vs. Babyschreien sind zwischen den beiden Teilkollektiven signifikant unterschiedlich $(\mathrm{p}<0,05)$. Während der Entspannungsmusik ist in beiden Gruppen ein Absinken auf annähernd Ausgangsniveau zu verzeichnen.

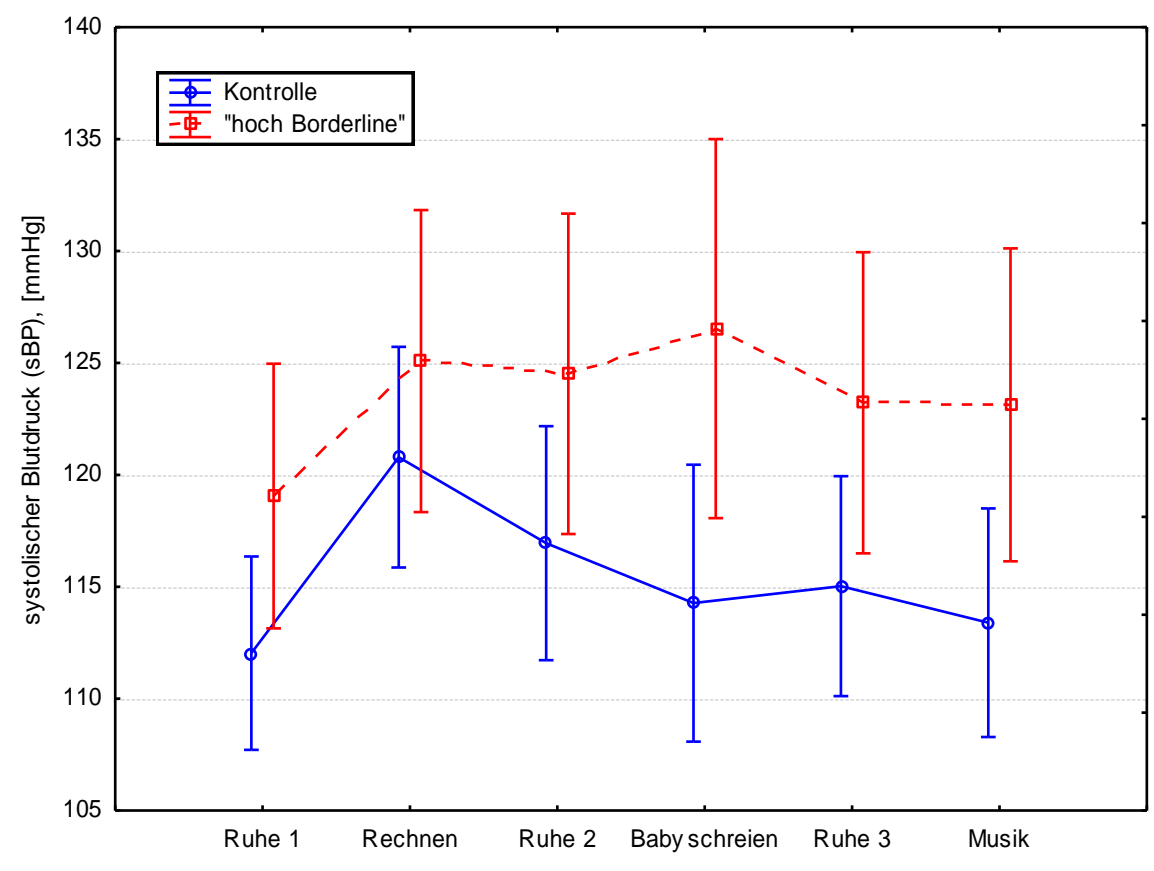

Abbildung 26: Verlauf systolischer arterieller Blutdruck (sBP) von Subgruppe „hoch Borderline“ und Vergleichsgruppe während der Stressbelastungsuntersuchung, Phasenmittelwerte und 95\%-Konfidenzintervalle 


\subsubsection{Barorezeptorsensitivität (BRS)}

Für die BRS total konnte ein fast signifikanter Gruppeneffekt in der Gegenüberstellung der Subgruppe „hoch Borderline“ und der Vergleichsgruppe gefunden werden ( $\mathrm{p}=0,051)$, wobei die hoch BorderlinePatientinnen eine durchgehend niedrigere BRS aufweisen (Abbildung 27, Seite 71). Die ermittelte globale Wechselwirkung erwies sich nach Adjustierung in den einzelnen Mittelwert-Vergleichen der Phasen zwischen den Gruppen als nicht signifikant.

Für den Ausgangsmittelwert der BRS total wurde für die Subgruppe 10,1 ms/mmHg ermittelt, der Vergleichswert der Kontrolle beträgt $12,1 \mathrm{~ms} / \mathrm{mmHg}$. Während des Rechentests sinkt die BRS in der Subgruppe auf $8,7 \mathrm{~ms} / \mathrm{mmHg}$ und in der Kontrollgruppe auf $10,8 \mathrm{~ms} / \mathrm{mmHg}$ ab. Während des Babyschreiens zeigt sich in der Vergleichsgruppe ein deutlicher Anstieg der BRS (13,7 ms/mmHg), während es in der Subgruppe ein leichtes Absinken der BRS im Vergleich zur Ruhephase 1 hervorruft $(9,1 \mathrm{~ms} / \mathrm{mmHg})$. Die Phasenmittelwert-Differenz zwischen Rechnen vs. Babyschreien beträgt in der Subgruppe 0,4 ms/mmHg und in der Vergleichsgruppe 2,8 ms/mmHg. Der Paarvergleich der beiden Stressoren (Rechnen vs. Babyschreien) offenbart somit fast signifikant unterschiedliche Änderungen zwischen den beiden Teilkollektiven für die BRS ( $\mathrm{p}=0,051$ ). Während der Entspannungsmusik nimmt die BRS sowohl in der Subgruppe $(10,9 \mathrm{~ms} / \mathrm{mmHg})$ als auch in der Kontrollgruppe $(16,3 \mathrm{~ms} / \mathrm{mmHg})$ maximale Phasenmittelwerte an.

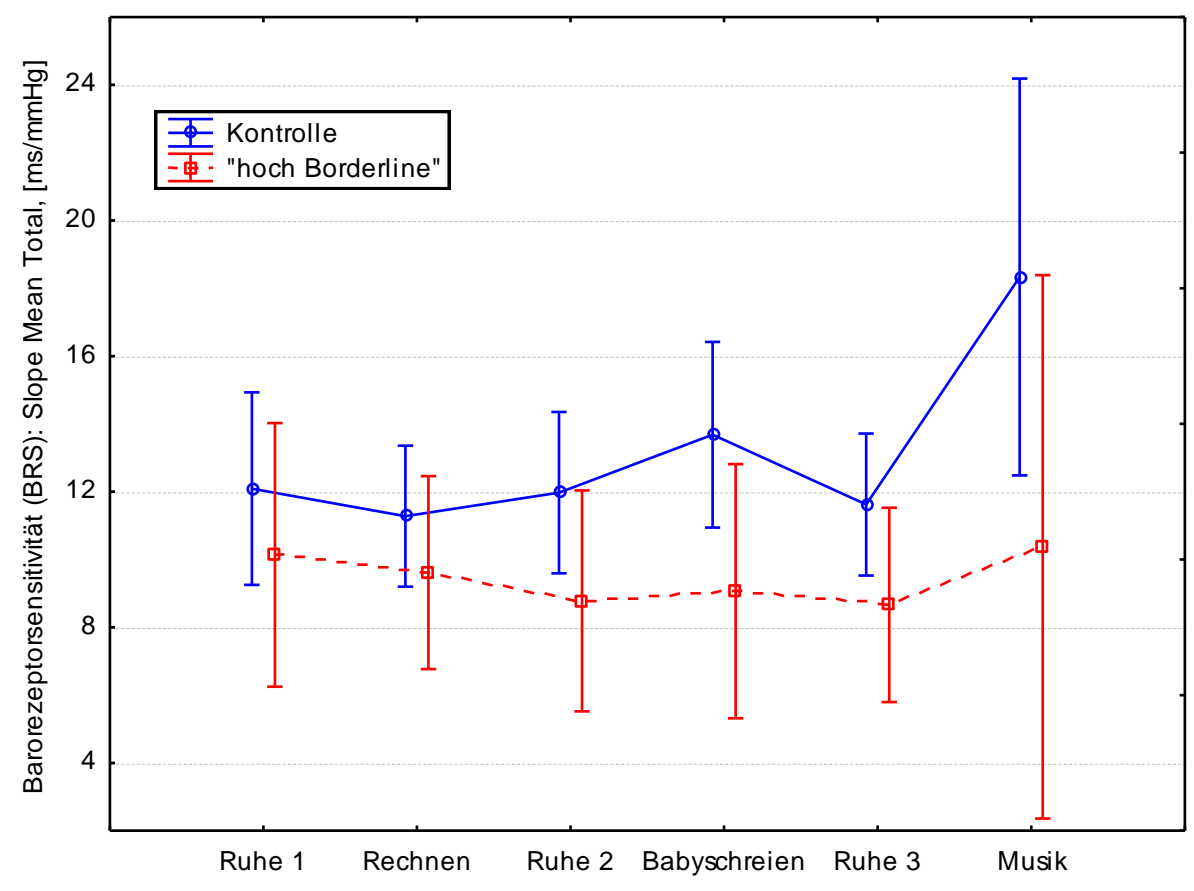

Abbildung 27: Verlauf Barorezeptorsensitivität (Slope Mean Total) von Subgruppe „hoch Borderline“6 und Vergleichsgruppe während der Stressbelastungsuntersuchung, Phasenmittelwerte und 95\%-Konfidenzintervalle 


\subsubsection{Verlauf der restlichen TFM-Parameter}

In der zweifaktoriellen ANOVA fanden sich für die restlichen TFM-Parameter CI, HR, TPRI, SDNN, HF-HRV und LF-HRV keine signifikanten Gruppen- und/oder Gruppen- mal Zeiteffekte. Im folgenden Abschnitt wird daher der Verlauf dieser Parameter sowie graphisch aufgefallene Gruppenunterschiede nur kurz beschrieben. Das Reaktionsverhalten (Zunahme $\uparrow$, Abnahme $\downarrow$ bzw. kaum Veränderung $\leftrightarrow$ ) aller TFM-Parameter während der Stresstests „Rechnen“ und „Babyschreien“ sowie signifikant unterschiedliche Phasenmittelwert-Differenzen des durchgeführten Paarvergleiches Rechnen vs. Babyschreien zwischen den Gruppen ist in Abbildung 25 (Seite 69) dargestellt.

Für die HR zeigt sich graphisch ein ähnlicher Verlauf in beiden Gruppen, wobei die Patientinnen der Subgruppe „hoch Borderline“ insgesamt etwas höhere HR-Werte aufweisen insbesondere während des Babyschreiens und in der nachfolgenden Erholungsphase (Ruhe 3).

Der Cardiac Index (CI) war bei ähnlichem, während der Stressbelastungsuntersuchung gleichbleibenden Verlauf in der Patientinnen-Untergruppe insgesamt etwas niedriger.

Der TPRI nimmt in der hoch Borderline-Gruppe insgesamt etwas höhere Werte an. Das Babyschreien führte in der Subgruppe zu einem Anstieg des TPRI, beim Rest des Ausgangskollektivs blieben die Werte auf Höhe der vorangegangen Ruhephase. Im Paarvergleich der beiden Stressoren (Rechnen vs. Babyschreien) fanden sich daher signifikant unterschiedliche Phasenmittelwert-Differenzen zwischen den Gruppen $(\mathrm{p}<0,05)$.

Ein ähnlicher Verlauf zwischen Subgruppe und Kontrolle zeigte sich für die SDNN, wobei die hoch Borderline-Patientinnen etwas kürzere RR-Intervalle aufweisen.

Die HF-HRV ist in der Subgruppe „hoch Borderline“ insgesamt etwas niedriger.

Die LF-HRV steigt während des Rechnens in der Kontrollgruppe graphisch stärker an und erzielt während dieses Tests auch höhere Werte. In beiden Gruppen wird während des Babyschreiens der minimale Phasenmittelwert bestimmt. 


\section{Diskussion}

Im Folgenden sollen die aufgeführten Ergebnisse zur psychophysiologischen Stressreagibilität bei Frauen mit (k)PTBS bzw. BPS im Kontext des aktuellen Forschungsstandes diskutiert werden. Nach einer allgemeinen Einordung des Patientinnenkollektivs mit Bezug zu den psychometrischen Daten, werden die einzelnen Abschnitte der Stressbelastungsuntersuchung (Rechentest, Babyschreien, Entspannungsmusik und Erholung) näher beleuchtet. Als Besonderheit der vorliegenden Studie wird eine spezifische Patientinnen-Subgruppe mit ausgeprägter Borderline-Symptomatik auf ihre Stressreagibilität hin mit den restlichen (k)PTBS-Patientinnen ohne Borderline-Merkmale verglichen. Abschließend werden Stärken und Schwächen der Arbeit erläutert sowie ein Ausblick und Anregungen für künftige Studien gegeben.

\subsection{Das Patientinnenkollektiv vor dem Hintergrund der Komplexität traumaassoziierter Störungsbilder}

Bei allen bisher vorliegenden Studien wurde nicht genau differenziert zwischen akuter PTBS, chronischer PTBS, komplexer PTBS und Borderline-Persönlichkeitsstörung, die oft auch zu den Traumafolgestörungen gerechnet wird. Insbesondere für die BPS besteht eine deutliche Überschneidung der Symptomatik mit einer kPTBS und auch mit dissoziativen Störungen (Sachsse et al. 1997, Driessen et al. 2002, Zanarini et al. 2006, Sack et al. 2012). Die überlappenden Störungsbilder könnten gemäß dem Konzept zweier Typen posttraumatischer Symptomatik in Form externalisierender und internalisierender Symptomatik (Miller und Resick 2007) als unterschiedliche Ausprägungen einer gemeinsamen Grundproblematik verstanden werden. Dabei entspräche die BPS dem externalisierenden Typus mit gestörter Impuls- und Affektregulation. Bei der internalisierenden Symptomatik dominieren hingegen depressive, dissoziative sowie vermeidende Verhaltensmuster, was mit der Diagnose einer kPTBS vereinbar wäre (Overkamp und Helmke-Jaeger 2010, Sack et al. 2012). Eine aktuelle Multi-Center-Studie hat ergeben, dass eine Differenzierung der diagnostischen Cluster kPTBS und BPS nicht möglich ist, beide Entitäten also vermutlich die gleiche Patientengruppe zu erfassen versuchen (Sack et al. 2012).

In der hier vorliegenden explorativen Pilot-Studie wurden insgesamt 54 Patientinnen mit einer Traumafolgestörung entsprechend der Ein- und Ausschlusskriterien in die Studie eingeschlossen. Es erfolgte eine Erhebung der Prävalenz von (k)PTBS, dissoziativen Störungen, BPS und anderen psychiatrischen Erkrankungen mit standardisierten Interviews (DDIS, IPDE, I-kPTBS) und Fragebogendiagnostik (IES-R, BSL, BPI, FDS, DES, BDI, SCL-90-R) auf der spezialisierten TraumaStation des AFG durch erfahrene Kliniker. Der Großteil der Studienteilnehmerinnen (79,6\%) erhielt die Diagnose chronifizierte PTBS nach Trauma Typ II (seltener nach Trauma Typ I), 20,4\% der Patientinnen erfüllten die Kriterien einer komplexen PTBS. 34 Patientinnen (63\%) litten zusätzlich an 
einer dissoziativen Symptomatik. Die (k)PTBS erfüllte bei 22 Patientinnen (40,7\%) auch die Kriterien einer emotional instabilen Persönlichkeitsstörung vom Borderline-Phänotyp. Anhand dieser Prävalenzzahlen wird die hohe Komorbiditätsrate der Traumafolgestörungen deutlich, wie sie bereits in zahlreichen Studien gefunden wurde (Sack et al. 2012, Dammann und Overkamp 2004, Zanarini et al. 2006). Aufgrund der bereits erläuterten weitreichenden Überlappung von Borderline- und Traumafolgesymptomen wurde auf eine weitere differentialdiagnostische Unterscheidung der posttraumatischen Störungsbilder verzichtet und das Gesamtkollektiv als solches untersucht. Eine differenzierte Gegenüberstellung akuter PTBS, chronischer PTBS und komplexer PTBS sowie eine weitere Klärung der diagnostischen Differenzierung von kPTBS und BPS bleibt künftigen Studien vorbehalten.

Dem aktuellen Stand der Forschung zur Traumaprävalenz an Patienten mit (k)PTBS bzw. BPS (Zanarini et al. 2006, Sack et al. 2012) entsprechend weist ein Großteil der hier untersuchten Patientinnen frühkindliche traumatisierende Erlebnisse auf (48 von 54, 88,9\%), wobei sexuelle Gewalterfahrungen $(74,0 \%)$ häufiger als körperliche Gewalterfahrungen $(63,0 \%)$ vertreten sind. Es überwiegen Typ-II-Traumata.

Das Spektrum der Nebendiagnosen im untersuchten (k)PTBS-Kollektiv reicht von depressiven und somatoformen Störungen über Angst- bzw. Panikstörungen bis hin zu Essstörungen, Adipositas und Hypertonie und entspricht damit dem typischen Bild an Komorbiditäten bei PTBS (Sachsse 2002, Sack et al. 2012). 40,7\% der Patientinnen zeigten selbstverletzendes Verhalten (SVV), welches bei Patienten mit (k)PTBS bzw. BPS oft beobachtet wird und neben dem Stressabbau und der Selbsttröstung vor allem auch der Beendigung dissoziativer Zustände und emotionaler Taubheit dient (Sachsse 2002). Passend zum bei PTBS weit verbreiteten Substanzmissbrauch findet sich im hier untersuchten Patientinnenkollektiv eine hohe Anzahl an Raucherinnen (57,4\%), Tablettenabhängigkeit $(16,7 \%)$ und Alkoholabusus in der Vorgeschichte (20,4\%). Erwartungsgemäß nehmen die Studienteilnehmerinnen mit (k)PTBS bzw. BPS zahlreiche Medikamente, schwerpunktmäßig Psychopharmaka, ein.

Zusammenfassend kann das hier untersuchte Patientinnenkollektiv sowohl hinsichtlich traumassoziierter und anderer Komorbiditäten als auch der Prävalenz Traumatisierungen im Kindesalter als typisches (k)PTBS-Kollektiv beurteilt werden.

\subsection{Die Stressbelastungsuntersuchung}

Mit Hilfe des Task Force Monitor, einer gut validierten, nicht-invasiven Messmethode zur Erfassung hämodynamischer und autonomer Parameter (Gratze et al. 1998, Fortin et al. 2001), war es möglich eine differenzierte Betrachtung der psychophysiologischen Reaktivität multidimensional durchzuführen. Während vorangegangene Studien nur einzelne Herzkreislauf-Parameter untersuchten, erfolgte hier eine Analyse sowohl vorwiegend beta- (HR und CI) und vorwiegend alpha-adrenerger (sBP und TPRI) als auch autonomer Parameter unterschiedlicher, insbesondere auch vagal regulierter 
Bereiche (SDNN, HF- und LF-Komponente der HRV sowie BRS). Des Weiteren erlaubte der Versuchsaufbau mit Ruhephasen und dem Einsatz zweier Stresstests (Rechnen und Babyschreien) die Beurteilung der Reagibilität in Ruhe, auf verschiedene Alltagsstressoren und die Rückbildungsfähigkeit nach Stressanforderung. Das Babyschreien wurde dabei unseres Wissens erstmals bei PTBS-Patienten als Stressor eingesetzt.

In den folgenden Abschnitten wird zunächst das Reaktionsverhalten der einzelnen TFM-Parameter während der zwei Stresstests, in den Erholungsphasen und während der Entspannungsmusik in Bezug zu anderen Studien gesetzt und eine mögliche Interpretation vorgenommen. Anschließend erfolgt die Betrachtung der Subgruppe „hoch Borderline“ im Vergleich zum Restkollektiv.

\subsubsection{Rechentest}

Als kognitiver Stressor diente ein bereits in zahlreichen Studien eingesetzter Rechentest (Kop et al 2004, Lache 2006), welcher hier unseres Wissens erstmals bei Patientinnen mit (k)PTBS bzw. BPS unter einem multidimensionalen Blickwinkel auf verschiedene Stressparameter hin untersucht wurde.

Der Rechentest ruft bei den Patientinnen im Vergleich zur Ruhephase (Baseline) eine autonome kardiovaskuläre Aktivierung hervor. Auf beta-adrenerger Ebene zeigt sich ein erwarteter signifikanter Anstieg der HR sowie eine Steigerung des CI. Die vorwiegend alpha-adrenerge Modulation ruft zwar einen signifikanten Anstieg des arteriellen Blutdrucks (sBP, dBP und mBP) hervor, die erwartete Zunahme des TPRI ist jedoch nicht signifikant. Die vorwiegend vagal bestimmten Parameter SDNN, HF-HRV und BRS sinken während des Rechnens ab, was einer Abnahme des vagalen Tonus entspricht. Diese ist jedoch statistisch nicht signifikant. Wie erwartet steigt die LF-HRV, als vorwiegend sympathisch modulierte HRV-Komponente, signifikant an. Das Reaktionsmuster während des Rechentests kann insgesamt als eine Aktivierung des Kampf-Flucht- bzw. Furcht-Systems mit Sympathikusanstieg und Vagusabfall interpretiert werden (Panksepp et al. 1991), wobei die vagale Modulation bei den PTBS-Patientinnen eingeschränkt zu sein scheint.

Analog zur physiologischen Aktivierung während des Rechentests zeigten die Patientinnen auch emotionale bzw. vegetative Reaktionen und gaben ein zunehmendes Stressempfinden auf einer Analogskala (SUD 0-10) an. Das mittlere Stresserleben während des Rechnens lag bei 6,3 und war damit signifikant im Vergleich zum Ausgangsstressniveau in Ruhe gestiegen. Somit konnte durch Simulation alltäglicher Stressbelastungen (Kopfrechnen) bei Patientinnen mit (k)PTBS bzw. BPS eine subjektive Aktivierung in Form erhöhter Mittelwerte auf der SUD im Vergleich zum subjektiven Stressempfinden in Ruhe induziert werden. Der Mittelwert dieses Patientinnenkollektivs ist vergleichbar mit Angaben vorheriger Studien, welche im Rahmen von traumaassoziierten Stimuli zwischen 5,8 und 6,9 auf der SUD lagen (Sack et al. 2004, Keane et al. 1998).

Bei einigen Patienten wurden passend zum angegebenen Stressempfinden während der Rechenaufgabe emotionale bzw. vegetative Reaktionen in Form von schwerer Atmung, Erregungszuständen, Weinen und das Nutzen von Copingmechanismen beobachtet. Eine Patientin zeigte ein Numbing-Verhalten, was dem Bereich der dissoziativen Stressbewältigungsstrategie zuzuordnen wäre. Zusätzlich beklagten 
einige Frauen Mundtrockenheit bzw. das Gefühl „einen Kloß im Hals zu haben“, dies könnte als eine zusätzliche Aktivierung des Paniksystems verstanden werden (Hüther und Sachsse 2007). Des Weiteren hatte ein Großteil der Patientinnen ausgeprägte Konzentrationsprobleme während des Kopfrechentests, was ein PTBS-Symptom (erhöhtes Arousal, Kriterium D) sein kann, aber natürlich nicht sein muss. Aus einer aktuellen Metaanalyse zu einem mittels EEG ermittelten speziellen evozierten Potentials (P300) bei PTBS-Patienten, zogen Karl et al. (2006b) die Schlussfolgerungen, dass die beobachteten erniedrigten P300-Potentiale auf neutrale Stimuli eine verminderte Konzentrationsfähigkeit abbilden und gleichzeitig erhöhte P300-Potentiale auf traumaassoziierte Stimuli eine gesteigerte Aufmerksamkeit widerspiegeln. Dieses Muster entspricht klinischen Angaben von Konzentrationsproblemen einerseits und Hypervigilanz andererseits unter relativ alltäglichen Stressbelastungen. Die in der vorliegenden Studie beobachteten Konzentrationsprobleme passen darüber hinaus zu neurobiologischen Erkenntnissen einer Hippokampusatrophie bei PTBS (für eine Übersicht siehe Karl et al. 2006a). Schädigungen der Hippokampusformation sind im Allgemeinen mit kognitiven Defiziten vergesellschaftet. Betroffen sind vor allem rechtshemisphärische Funktionen der Aufmerksamkeit, des visuellen Gedächtnisses sowie der Handlungsintelligenz (Weniger et al. 2008). Verminderte Volumina von Hippokampus und Amygdala sowie kognitive Defizite konnten sowohl für die PTBS als auch für eine traumabedingte kPTBS/BPS nachgewiesen werden (Schmahl et al. 2003, Irle et al. 2009). Im Gegensatz dazu scheinen Patienten, die in Folge einer Traumatisierung eine dissoziative Störung entwickeln, keine Hippokampus- bzw. Amygdala-Atrophie aufzuweisen. Es bestehen bei hoch dissoziativen Patienten auch keine kognitiven Minderleistungen (Irle et al. 2009, Weniger et al. 2008). Anhand dieser Befunde ist es offensichtlich, dass bei den Traumafolgestörungen eine klare Differenzierung einer komorbiden Dissoziation erfolgen muss. Hier liegt möglicherweise auch ein Erklärungsansatz für die individuell so unterschiedlichen Reaktionsmuster während der Stressbelastungsuntersuchung und warum nicht alle Patientinnen mit (k)PTBS Konzentrationsschwierigkeiten während des Rechentests aufweisen. Zudem ist die bekannte Hippokampusatrophie bei chronifizierter PTBS eine statistische Verschiebung des Mittelwertes, keine monokausale Verbindung; es gibt also durchaus Menschen mit chronischen PTBS-Symptomen, die keine Hippokampus-Atrophie aufweisen. Bei dem hier untersuchten Klientel wurde bekanntlich keine Hirndiagnostik durchgeführt.

\subsubsection{Hämodynamische Parameter während Rechnen}

Einer der am besten beforschten Herzkreislauf-Parameter bei PTBS-Patienten sowohl in Ruhe als auch unter Stresseinwirkung ist die HR (Literatur siehe unten). Dieser sowohl beta-adrenerg als auch vagal modulierte Parameter wurde in der vorliegenden Studie durch den bisher kaum berücksichtigten CI ergänzt. Unter Ruhebedingungen wiesen die PTBS-Patientinnen bereits eine mittlere HR von 76/min auf. Während des Rechentests stieg die HR erwartungsgemäß an und erreichte sogar den höchsten Phasenmittelwert der gesamten Stressbelastungsuntersuchung. Der Unterschied zwischen Baseline und Rechnen war statistisch signifikant, sodass von einer adäquaten HR-Regulation bei den 
Patientinnen ausgegangen werden kann. Eine exakte Zuordung zu einer spezifischen Komponente des ANS ist für die hier gewählten prototypischen Parameter HR und CI nicht eindeutig möglich.

Eine aktuelle Metaanalyse von Pole (2007) offenbarte bisher inkonsistente Ergebnisse bezüglich der Ruhe-HR bei PTBS mit Tendenz zu einer Erhöhung. Viele Studien fanden eine erhöhte HR in Ruhe bei PTBS-Patienten (u.a. Cohen et al. 1997, Keane et al. 1998, Metzger et al. 1999). In manchen Studien wurde jedoch kein Unterschied der HR in Ruhe beim Vergleich von PTBS-Patienten und Gesunden gesehen (u.a. Sahar et al. 2001). Unter Einbeziehen der respiratorischen Sinusarrhythmie (RSA) machen neuere Studien einen defizitären Parasympathikus mitverantwortlich für eine erhöhte Ruhe-Herzfrequenz bei PTBS (Sack et al. 2004, Hopper et al. 2006), sodass nicht bei allen Patienten mit PTBS eine erhöhte HR zu erwarten ist, sondern nur wenn ein erniedrigter Vagotonus in Ruhe vorliegt. Pole (2007) untersuchte weiterhin Studien zur Reaktivität auf verschiedene neutrale und traumaassoziierte Stimuli bei PTBS. Es zeigte sich eine erhöhte Schreckreaktion auf erschreckende Töne mit einer stärkeren Reaktivität von HR, Hautwiderstand und M. orbicularis oculi-EMG sowie eine langsamere Habituation (Shalev et al. 1992, Metzger et al. 1999). Auch auf standardisierte und individuelle traumaassoziierte Bilder reagierten die PTBS-Patienten mit einer stärker ausgeprägten Aktivierung mit deutlichem Anstieg der HR im Vergleich zu Gesunden bzw. Traumatisierten ohne PTBS (Blanchard et al. 1996, Keane et al. 1998, Ehlers et al. 2010). Erinnerungen an das Trauma lösen demnach ähnliche Reaktion wie das Trauma selbst aus (Triggerwirkung), was auf einer operanten Konditionierung in Zusammenspiel mit Vermeidungsverhalten beruht. In Widerspruch zu diesen Ergebnissen fand die Arbeitsgruppe Cohen et al. (1998, 2000) während eines Trauma Recall keinen weiteren Anstieg der HR bei PTBS-Patienten, in der Vergleichsgruppe hingegen stieg die HR an. Cohen et al. führten dies auf eine bereits in Ruhe erhöhte HR als Zeichen einer chronischen sympathovagalen Imbalance bei PTBS ohne Reaktionsfähigkeit unter Stressanforderung zurück. Diese These konnte jedoch in der vorliegenden Studie für den trauma-neutralen Stressor „Rechentest“ nicht bestätigt werden.

Wenige Studien setzten bisher einen Kopfrechentest zur Beurteilung der Reaktivität der HR bei PTBS ein. Der signifikante Anstieg der HR während des hier eingesetzten Kopfrechentests entspricht den Beobachtungen vorangegangener Studien, in denen diese Form der mentalen Stressanforderung ebenfalls eine HR-Steigerung bei PTBS-Patienten bewirkte (Blanchard et al. 1996, Keane et al. 1998, Keary et al. 2009). Blanchard et al. (1996) konnten keinen Unterschied in der HR-Reaktion (Anstieg) zwischen Motorradunfallopfern mit und ohne PTBS nachweisen. Ähnliche Ergebnisse wie die Arbeitsgruppe Blanchard et al. (1996) konnten durch Keary et al. (2009) an Frauen mit PTBS repliziert werden, die ebenso wie die Vergleichsgruppe einen Anstieg der HR während eines Rechentests aufwiesen. Keane et al. (1998) hingegen fanden eine geringer ausgeprägte Steigerung der HR während eines Rechentests bei Kriegsveteranen mit PTBS. Das Antwortverhalten der HR auf traumaassoziierte Stimuli kann eventuell auch zur PTBS-Diagnostik herangezogen werden (Blanchard et al. 1996, Keane et al. 1998, Orr und Roth 2000). 
Analog zur HR stieg der CI während des Kopfrechentests an. Dieser Anstieg erreichte auch im Paarvergleich statistische Signifikanz. Bisher gibt es kaum Studien, die eine Impedanzkardiographie bei PTBS-Patienten durchführten, um Veränderungen des Herzzeitvolumens bzw. des CI während einer Stressanforderung $\mathrm{zu}$ beurteilen. Peckerman et al. (2003) fanden einen Anstieg des Herzzeitvolumens während des Berichtens einer unangenehmen Situation, nicht jedoch während eines Rechentests, was in Widerspruch zu den vorliegenden Ergebnissen steht.

Wie die vorliegende Studie zeigen konnte, führen auch vermeintlich neutrale Alltagsstressoren wie ein Rechentest bei Patientinnen mit (k)PTBS bzw. BPS zu einem Anstieg von HR und CI. Dies kann als intakte Regulationsfähigkeit der vagal bzw. beta-adrenerg modulierten Herzkreislauf-Parameter HR und CI in Stresssituationen bei PTBS im Sinne einer Kampf-Flucht Reaktion verstanden werden.

Während der arterielle Blutdruck in einigen Studien untersucht wurde, war der periphere Gefäßwiderstand kaum Gegenstand der bisherigen Forschung zur Stressphysiologie bei PTBS.

Der sBP - stellvertretend für alle drei erhobenen Blutdruckwerte - stieg während des Kopfrechnens signifikant an, was als klassische Stressreaktion verstanden werden kann. Der dementsprechend ursächlich zu erwartende Anstieg des totalen peripheren Widerstands-Index (TPRI) fiel gering aus, sodass der Unterschied zwischen den Phasenmittelwerten des TPRI in Ruhe und während des Rechentests keine statistische Signifikanz erreichte. Hier scheint eine veränderte Regulationsfähigkeit des peripheren Gefäßwiderstands unter mentaler Stressanforderung vorzuliegen. Dies entspricht Befunden von Peckerman et al. (2003), die bei Kriegsveteranen mit chronischer Fatigue und komorbider PTBS keinen Anstieg des totalen peripheren Widerstands sowohl während des Berichtens einer unangenehmen Situation als auch während eines Kopfrechentests im Vergleich zu Veteranen ohne PTBS-Symptomatik sowie gesunden Kontrollprobanden fanden. Zwar wurde für beide Stressoren ein Anstieg des sBP und dBP gesehen, dieser war jedoch in der PTBS-Gruppe geringer ausgeprägt. Ähnliche Ergebnisse einer geringer ausgeprägten Blutdrucksteigerung, insbesondere des dBP, bei PTBS-Patienten im Vergleich zu gesunden Kriegsveteranen während eines Rechentests berichteten bereits Keane et al. (1998). Auch die Arbeitsgruppe Blanchard et al. (1996) fand bei Motorradunfallopfern mit PTBS einen geringeren Anstieg des sBP als bei solchen ohne PTBS (Blanchard et al. 1996). Inkonsistent zu diesen Beobachtungen konnte in einer neueren Studie von Keary et al. (2009) jedoch kein signifikanter Unterschied in der Blutdruckreaktion von Frauen mit und ohne PTBS sowohl auf einen neutralen (Rechnen) als auch auf einen traumaassoziierten Stressor (Trauma Recall) gesehen werden. In beiden Gruppen stieg sBP und dBP während der Stressanforderung gleichermaßen an. Keane et al. (1998) wiesen wiederum eine stärkere Blutdruckreaktivität von PTBS-Patienten im Vergleich zu Gesunden auf traumaassoziierte Stimuli nach. Auch in einer aktuellen Metaanalyse von Pole (2007) stellte sich eine erhöhte Reaktivität des dBP auf traumaassoziierte Stressoren bei PTBS heraus. Während Pole auch erhöhte Blutdruckwerte (sBP und dBP) bei PTBS-Patienten in Ruhe herauskristallisierte, fanden Hughes et al. (2007) keinen 
Unterschied des Ruhe-Blutdrucks im Vergleich zu Gesunden. Im Rahmen eines Orthostasestests zeigten PTBS-Patienten eine eingeschränkte Blutdruckregulation mit einem steileren Abfall des sBP, was auf eine ausgeprägte Vasodilatation durch einen im Vergleich stärkeren Abfall des TPR zurückzuführen war und mit einer kurzfristigen Steigerung des Herzauswurfvolumens einherging, sowie eine langsamere Erholungs-Zeit nach dem plötzlichen Aufstehen (Peckerman et al. 2003). Peckerman et al. (2003) vermuten eine gestörte Integrität höherer Hirnstrukturen zur zentralen Regulation vasomotorischer Aktivität als Ursache für die gesehene Hyporeaktivität auf mentale Stressoren bei PTBS. Walczewska et al. (2011) fanden darüber hinaus einen Zusammenhang zwischen dem PTBS-Schweregrad und einer erhöhten Pulswellengeschwindigkeit (Pulse Wave Velocity PWV), als Index einer erhöhten Steifigkeit der Arterien. Ein chronisches Hyperarousal, wie es für die PTBS typisch ist, kann demnach zu ausgeprägten Blutdruckschwankungen mit hypertensiven Episoden führen und letztendlich eine Steifigkeit der Gefäße begünstigen (Walczewska et al. 2011). Diese Ergebnisse passen zur hohen Prävalenzrate an arterieller Hypertonie im hier untersuchten (k)PTBSPatientinnenkollektiv.

Trotz zum Teil inkonsistenter Studienergebnisse der Vergangenheit scheint in der Zusammenschau mit den aktuellen Ergebnissen dieser Studie zwar ein Anstieg des Blutdrucks, jedoch eine eingeschränkte Reaktivität des peripheren Gefäßwiderstandes auf mentale Stresseinwirkung (Rechnen) bei PTBS vorzuliegen.

\subsubsection{Autonome Parameter während Rechnen}

Die HRV gilt als Maß für die individuelle Fähigkeit sich an stressvolle Situationen anzupassen und ist in den letzten Jahrzehnten vermehrt in Bezug auf Stressfolgestörungen wie der PTBS erforscht worden. Die Standardabweichung aller RR-Intervalle (SDNN) als Maß für die Gesamtvariabilität der HRV, welche im Zeitbereich direkt bestimmt wird, wurde dabei jedoch bisher im Kontext von verschiedenen Stresseinwirkungen kaum betrachtet. Die Powerspektralanalyse der HRV hingegen wurde in einigen Studien an PTBS-Patienten ausgewertet. Hierbei standen insbesondere die HF- und LF-HRV sowie die RSA im Vordergrund. Im Rahmen der vorliegenden Stressbelastungsuntersuchung bei Frauen mit (k)PTBS bzw. BPS wurden daher sowohl die SDNN (Zeitbereich) als auch die HF- und LF-HRV (Frequenzbereich) sowie als ergänzender autonomer Parameter die BRS untersucht.

Während des Rechentests zeigte sich eine Abnahme der SDNN. Diese war statistisch jedoch nicht signifikant. Es liegt hier eine stressbezogene Modulation des ANS vor, die sowohl sympathische als auch parasympathische Einflüsse widerspiegelt. Eine erniedrigte SDNN ist als eine Dysregulation des autonomen Nervensystems mit eingeschränkter vagaler Modulation zu verstehen (Task Force 1996). Bei Kriegsveteranen mit PTBS wurde eine herabgesetzte HRV in Form einer erniedrigten SDNN, gemessen über einen Zeitraum von 5 Minuten, in Ruhe im Vergleich zu Veteranen ohne PTBSDiagnose gefunden (Tan et al. 2011). Auch Motorradunfallopfer mit PTBS-Symptomatik weisen eine herabgesetzte SDNN auf (Shaikh al arab et al. 2012). Eine bereits in Ruhe erniedrigte SDNN bei Vorliegen einer PTBS könnte eine Erklärung für ein eingeschränktes Absinken während einer 
Stressanforderung ohne signifikanten Unterschied zur Baseline darstellen. Oder aber es zeigt sich hier eine eingeschränkte Regulationsfähigkeit der RR-Intervalle bei PTBS.

Eine Unterteilung der sympathischen und parasympathischen Einflüsse auf die HRV ist mit Hilfe einer Frequenzdichteanalyse möglich. Der Vagotonus kann hierbei durch die HF-HRV $(0.15-0.4 \mathrm{~Hz})$ oder die RSA $(0.12-0.4 \mathrm{~Hz})$ abgebildet werden. Die LF-HRV $(0.04-0.15 \mathrm{~Hz})$ spiegelt in erster Linie sympathisch vermittelte Oszillationen wider, sie unterliegt jedoch auch einem über den Baroreflex vermittelten vagalen Einfluss und gibt daher Auskunft sowohl über den sympathischen als auch den vagalen Tonus (Task Force 1996). Als Zeichen eines stressbedingten Abfalls der Vagusaktivität zeigte sich ein erwarteter Abfall der HF-HRV während des Kopfrechentests, welcher jedoch statistisch nicht signifikant war. Analog zu dieser Beobachtung stieg die LF-HRV während des Rechnens deutlich an. Dieser Anstieg erreichte einen signifikanten Unterschied der Phasenmittelwerte der LF-HRV zwischen Baseline und Rechentest. Hier stellt sich eine typische Stressreaktion mit Aktivierung des Sympathikus und Absinken des parasympathischen Tonus dar. Zum Thema HRV bei PTBS gibt es derzeit zwei unterschiedliche Auffassungen:

- Auf der einen Seite postuliert die Arbeitsgruppe Cohen et al. (1997, 1998, 2000) eine erniedrigte Parasympathikus-Aktivität (erniedrigte HF-HRV) mit gleichzeitig gesteigerter Sympathikus-Wirkung (erhöhte LF-HRV) bei PTBS-Patienten in Ruhe sowie eine fehlende Reaktivität unter Stressanforderung.

Aufgrund des Hyperarousal in Ruhe zeigt sich bei Stressbelastung kein Unterschied der HRV-Werte zwischen Gesunden und PTBS-Patienten. Während die HF-HRV in der Kontrollgruppe beim Berichten eines beängstigenden Erlebnisses absinkt, zeigt sich bei den PTBS-Patienten kein weiteres Absinken der ohnehin bereits erniedrigten Vagusaktivität während eines Trauma Recall (Cohen et al. 1998, 2000). Die PTBS-Patienten zeigen demnach in Ruhe ein ähnliches autonomes Aktivitätsmuster wie gesunde Probanden unter Einfluss eines stressreichen Stimulus. Cohen et al. (1998) sehen darin die Unfähigkeit zur physiologischen Stressantwort aufgrund einer chronischen autonomen Überstimulation. Diese Unfähigkeit auf einen stressvollen Stimulus mit autonomer Modulation zu reagieren, konnte auch in der Abgrenzung zu Patienten mit einer Panikstörung gefunden werden (Cohen et al. 2000). Zwar hatten Patienten mit einer Panikstörung ebenfalls eine erhöhte LFKomponente sowie eine erniedrigte HF-Komponente der HRV in Ruhe, sie zeigten jedoch eine signifikante Stressantwort für beide Komponenten während des Berichtens einer Panikattacke. Blechert et al. (2007) fanden beim Vergleich von PTBS-Patienten und Patienten mit Panikstörung auch in Ruhe einen erniedrigten Vagotonus (erniedrigte RSA) sowie eine erhöhte sympathische Aktivität (erhöhte elektrodermale Leitfähigkeit) in der PTBS-Gruppe. Unter Androhung eines kleinen Elektroschocks als Stresstest hatten die PTBS-Patienten eine abgestumpfte elektrodermale Antwort, was der oben beschriebenen Interpretation von Cohen et al. (1998) entspricht. Die Ergebnisse der vorliegenden Studie zeigen graphisch einen Abfall des Vagotonus und einen signifikanten Anstieg der Sympathikus-Aktivität während eines Rechentests und konnten daher die These einer fehlenden 
Reaktivität auf Stress bei PTBS nicht bestätigen. Da der Unterschied zwischen Baseline und Rechnen für die HF-HRV und SDNN jedoch nicht signifikant war, kann eventuell auf eine eingeschränkte vagale Modulation bei intakter Sympathikus-Reaktivität geschlossen werden.

- Im Gegensatz zur Theorie einer autonomen Überstimulation in Ruhe bei PTBS-Patienten mit fehlender Reaktivität auf Stress (Cohen et al. 1997, 1998) beschreiben andere Forscher keinen Unterschied der HRV in Ruhephasen im Vergleich zu Gesunden, sondern eine erhöhte Reaktivität auf Stressoren.

In einer aktuellen Studie von Keary et al. (2009) beispielsweise zeigte sich kein signifikanter Unterschied für die HRV-Komponenten HF- und LF-HRV im Vergleich von Frauen mit und ohne PTBS in Ruhe. Auch Sahar et al. (2001) fanden in Ruhe keinen Unterschied der RSA, die dem Hochfrequenzband der HRV zuzuordnen ist, zwischen PTBS-Patienten und Trauma-Exponierten ohne PTBS. Als Stressoren setzten Keary et al. (2009) sowohl einen Rechentest als auch ein Trauma Recall ein. Die Patientinnen reagierten ähnlich wie die Kontrollgruppe auf beide Stressoren mit einem Absinken der HF-HRV und einem entsprechendem Anstieg der LF-Komponente, was dem Reaktionsmuster des hier untersuchten Patientinnenkollektivs annähernd entspricht. Keary et al. (2009) wiesen in der PTBS-Gruppe aber einen deutlich stärkeren, im Vergleich zur Kontrolle signifikanten Abfall der HF-HRV unter Stressanforderung nach. Auch in der Studie von Sahar et al. (2001) rief ein Rechentest einen größeren Rückgang der Vagusaktivität (RSA-Abfall) bei Männern mit PTBS im Vergleich zu Kontroll-Traumatisierten ohne PTBS hervor. Während in der Vergleichsgruppe der Index für den Vagotonus (RSA) mit Veränderungen der HR korrelierte, zeigte sich in der PTBSGruppe eine Entkopplung von RSA und HR, die möglicherweise für eine gestörte Selbstregulation während mentaler Herausforderung verantwortlich ist (Sahar et al. 2001). Studien von Sack et al. (2004) und Hopper et al. (2006) machen darauf aufmerksam, dass nicht alle Patienten ein PTBStypisches Hyperarousal mit erhöhter Ruhe-Herzfrequenz aufweisen, sondern dass diese mit dem Vagotonus (RSA) zusammenhängt. Nur Subgruppen mit niedriger Baseline-RSA weisen eine erhöhte HR in Ruhe auf (Sack et al. 2004, Hopper et al. 2006). Patienten mit niedriger RSA zeigen zudem einen verlängerten Anstieg der HR mit langsamerer Erholungs-Zeit während eines traumaassoziierten Stressors. Ein herabgesetzter Vagotonus könnte somit als eine Ursache - zusätzlich zu einer erhöhten Sympathikusaktivität - für ein gestörtes Hyperarousal mit fehlender inhibitorischer Kontrolle der HR und eine eingeschränkte Emotionsregulation bei PTBS angesehen werden (Sack et al. 2004).

In der vorliegenden Studie konnte eine Anpassungsfähigkeit der HRV auf einen Rechentest bei (k)PTBS-Patientinnen gezeigt werden, wobei nur der Anstieg der LF-Komponente signifikant war. Die Modulationsfähigkeit des vagalen Tonus könnte demnach eingeschränkt sein.

Zur Ergänzung der autonomen Parameter wurde auch die BRS ausgewertet, die bisher nur von einer Forschungsgruppe (Hughes et al. 2006, 2007) bei PTBS-Patienten untersucht wurde. Die BRS sank während des Rechentests auf den minimalen Phasenmittelwert der Stressbelastungsuntersuchung ab. Jedoch war der Unterschied zur Baseline analog zu den Ergebnissen für SDNN und HF-HRV 
statistisch nicht signifikant. Niedrige BRS-Werte sind in erster Linie auf eine Abnahme des Vagotonus zurückzuführen (La Rovere et al. 1995). Der Rechentest löst bei den Patienten eine stressbezogene Modulation mit erniedrigter Parasympathikus-Aktivität aus, die jedoch lediglich eine geringe Effektstärke $(0,16)$ und keine statistische Signifikanz erreicht. Die vagale Modulationsfähigkeit scheint hier demnach eingeschränkt zu sein scheint. Die Arbeitsgruppe Hughes et al. (2007) beobachtete niedrigere BRS-Werte bei PTBS-Patienten im Vergleich $\mathrm{zu}$ gesunden Probandinnen sowohl in Ruhe als auch während einer Ärgerexposition (Anger Recall). Zwar zeigte die Kontrollgruppe einen stärken Abfall der BRS von Baseline zu Stressor (Anger Recall), dies führten Hughes et al. jedoch auf den signifikant niedrigeren Ausgangswert der PTBS-Patientinnen zurück. Eine erniedrigte Ruhe-BRS bei PTBS-Patienten hatten Hughes et al. bereits in einer Vorläuferstudie im Jahre 2006 gefunden. Diese Tatsache könnte auch eine mögliche Erklärung sein, warum der hier beobachtete Unterschied zwischen Baseline und Stressor „Rechentest“" keine statistische Signifikanz erzielte. Oder aber PTBS-Patientinnen weisen unter Stressanforderung eine eingeschränkte parasympathische Herabregulation auf, wie es bereits für die SDNN und HF-HRV diskutiert wurde. Eine erniedrigte BRS scheint PTBS-spezifisch zu sein, denn Hughes et al. (2007) konnten keine Unterschiede in der Ruhe-BRS zwischen PTBS-Patientinnen mit und ohne komorbide depressive Störung nachweisen. Auch die für die PTBS oft nachgewiesene erhöhte Ruhe-Herzfrequenz könnte durch eine erniedrigte BRS als Index einer erniedrigten Vagusaktivität bedingt sein (Hughes et al. 2007). Es bleibt zu überprüfen, ob die PTBS für eine erniedrigte BRS verantwortlich ist oder ob eine niedrige BRS als Prädiktor für eine PTBS angesehen werden muss.

Zusammenfassend konnte in der vorliegenden Studie gezeigt werden, dass ein Rechentest bei Patientinnen mit (k)PTBS bzw. BPS eine subjektive, emotionale sowie psychophysiologische Aktivierung auf verschiedenen Ebenen hervorrufen kann. Analog zu vielen Studien zur HRReaktivität bei PTBS wurde während des Rechnens ein signifikanter HR-Anstieg gesehen. Als ergänzender Parameter der beta-adrenergen Aktivierung hat sich hier auch der bisher kaum bei PTBS untersuchte $\mathrm{CI}$ bewährt. Auf beta-adrenerger Ebene (HR und CI) zeigt sich keine Einschränkung in der Regulationsfähigkeit auf den kognitiv-mentalen Stressor „Kopfrechnen“ bei Patientinnen mit (k)PTBS bzw. BPS. Die Ergebnisse für die alpha-adrenerge Modulation lassen eine eingeschränkte Regulationsfähigkeit für den peripheren Gefäßwiderstand vermuten, denn dieser nahm unter Stresseinwirkung entgegen den Erwartungen nicht signifikant zu. Für den arteriellen Blutdruck (sBP, $\mathrm{dBP}$ und $\mathrm{mBP}$ ) konnte aber ein adäquater Anstieg (offenbar vorwiegend durch den erhöhten CI bedingt) verzeichnet werden. Für die autonomen Parameter zeigt sich zwar eine erwartete Abnahme des Vagotonus während des Rechentests, diese fällt jedoch sowohl für SDNN, HF-HRV als auch BRS statistisch nicht signifikant aus. Hier lässt sich eine eingeschränkte vagale Modulation während einer mentalen Stressanforderung bei PTBS vermuten. Der Sympathikus hingegen scheint auch bei PTBSPatientinnen eine adäquate Regulationsfähigkeit aufzuweisen, wobei zu berücksichtigen ist, dass die 
LF-HRV nicht als rein sympathisch modulierte HRV-Komponente interpretiert werden darf. Es wird deutlich, dass nicht allein eine gesteigerte sympathische Aktivierung für das PTBS-spezifische Hyperarousal verantwortlich ist, sondern es ist zu vermuten, dass defizitäre parasympathische Einflüsse eine entscheidende Rolle in der Pathophysiologie der PTBS spielen.

\subsubsection{Babyschreien}

Als zweiter Stresstest diente eine Tonbandaufnahme mit Babyschreien, welcher unseres Wissens bisher nicht im Rahmen von psychophysiologischen Studien an PTBS-Kollektiven eingesetzt wurde.

Babyschreien zählt zu den sogenannten Distress Vocalisations, die zum Ziel haben bei der Mutter eine Stresssituation mit physiologischer Aktivierung auszulösen, welche sie durch Zuwendung zum Kind und Fürsorge beenden kann (Panksepp et al. 1991, Del Vecchio et al. 2009).

Ebenso wie der Rechentest ruft auch das Babyschreien bei den PTBS-Patientinnen insbesondere subjektiv ein Arousal hervor, wobei sich jedoch deutliche Unterschiede für einzelne hämodynamische sowie autonome Parameter zwischen den beiden Stressoren herausstellten. Unter Babyschreien kommt es im Vergleich zum Kopfrechnen wie erwartet zu einer signifikant stärkeren Zunahme des TPRI mit einhergehender Blutdrucksteigerung. Interessanterweise wurden für die HR und für den CI während des Babyschreiens eine Abnahme bzw. annähernd gleiche Werte im Vergleich zum Ausgangswert in Ruhe beobachtet. Hier zeigt sich in der vorliegenden Untersuchung ein signifikant unterschiedlicher Effekt der beiden Stressoren auf die vorwiegend beta-adrenerg modulierten hämodynamischen Parameter, die während des Rechentests angestiegen waren. Während die SDNN wie erwartet leicht absinkt, bleibt die HF-HRV auf Ausgangsniveau und für die weiteren autonomen Parameter zeigt sich ein gegensätzliches Reaktionsverhalten während des Babyschreiens im Vergleich zum Rechentest. Die Phasenmittelwerte der BRS steigen an und die LF-HRV sinkt, was einer Zunahme der vagalen bzw. einer Abnahme der sympathischen Aktivität entspricht. Jedoch waren diese Veränderungen der autonomen Parameter nicht signifikant im Vergleich zur Ruhephase 1. Im Paarvergleich zwischen den beiden Stressoren zeigte sich aber ein signifikanter Unterschied von LF-HRV und BRS. Somit wird beim Babyschreien eine andere Achse der autonomen Aktivierung stimuliert, die in den folgenden Abschnitten näher erläutert und diskutiert werden soll.

Auch das Babyschreien führte zu einer erwarteten, hochsignifikanten Zunahme des subjektiven Stressempfindens im Vergleich zum Ausgangsstressniveau in Ruhe. Jedoch fiel die subjektive Aktivierung während des Babyschreiens nicht wie erwartet stärker als während der Rechenaufgabe aus, sondern die Mittelwerte waren mit 6,3 auf der Analogskala identisch. Die Patientinnen bewerteten das Babyschreien aber deutlich häufiger mit dem Maximalwert von 10 Punkten auf der Analogskala. Dies passt zu subjektiven Bewertungen von Videoaufnahmen schreiender Kinder, die bei Erwachsenen ein stärkeres Gefühl von Feindseligkeit, Gereiztheit, Traurigkeit, Verzweiflung und Empathie als Aufnahmen lachender Kinder auslösten (Brewster et al. 1998). Für vier Patientinnen war die Stressbelastung durch das Babyschreien in der vorliegenden Studie sogar so groß, dass sie diesen 
Stresstest vorzeitig abbrechen mussten. Im Vergleich dazu führte der Rechentest nur bei einer Patientin zum Abbruch.

Im Unterschied zum Rechentest löste das Babyschreien auch eine deutlich stärker ausgeprägte emotionale bzw. vegetative Begleitsymptomatik in Form von schwerer Atmung, Erregungszuständen, Weinen, das Nutzen von Copingmechanismen sowie Numbing-Verhalten aus. Der Unterschied erwies sich für die Reaktionsformen schwere Atmung und das Nutzen von Copingmechanismen als statistisch signifikant und für den Erregungszustand zeigte sich eine statistische Tendenz. Die vermehrt beobachtete vegetative Komponente (Atmung, Erregung, Weinen) deutet auf eine stärkere emotionale Belastung durch das Babyschreien im Vergleich zum Rechentest hin und führt vermutlich zum häufigeren Einsatz von Kompensationsmechanismen (Copingstrategien, Numbing). Es gibt zahlreiche selbstschützende und selbstregulierende Manöver, die PTBS-Patienten nutzen, um sich aus der Stresssituation auszuklinken und somit auch ggf. die physiologische Reaktivität abzuschwächen. Es wird vermutet, dass Patienten, die auf ein Trauma mit Numbing, Freezing, Dissoziation und/oder einem physiologischen Hypoarousal reagieren auch in Stressbelastungsuntersuchungen eine gedämpfte psychophysiologische Reaktivität aufweisen (Lanius et al. 2006). Ein vermehrtes NumbingVerhalten während des Babyschreiens könnte demnach als mögliche Ursache für das vom Rechentest abweichende Reaktionsmuster angenommen werden.

In einer Korrelationsanalyse zeigte sich für die Anzahl der beobachteten vegetativen bzw. emotionalen Reaktionen während des Babyschreiens eine Korrelation mit der HR von 0,44. Des Weiteren korrelierten die autonomen Parameter negativ mit der Gesamtanzahl an Reaktionsformen (BRS -0,40, HF-HRV -0,36, LF-HRV -0,30). Dies zeigt, dass eine ausgeprägte vegetative Symptomatik mit einer erhöhten HR, einer herabgesetzten HRV und erniedrigtem Vagotonus einhergeht bzw. ein eingeschränkter vagaler Einfluss eine vegetative Begleitsymptomatik begünstigt.

\subsubsection{Hämodynamische Parameter während Babyschreien}

Das Babyschreien führte im Vergleich zum Ausgangswert in Ruhe nicht wie erwartet zu einem Anstieg von HR und CI sondern sogar zu einer geringfügigen, nicht signifikanten Abnahme. Hieraus ergibt sich ein signifikanter Unterschied zwischen den beiden Stressoren „Rechnen“ und „Babyschreien“" mit gegensätzlichem Reaktivitätsverhalten dieser Parameter.

Babyschreien löst normalerweise bei Erwachsenen ein physiologisches Arousal aus, welches zu einer sofortigen Zuwendung zum Kind führt, um das Schreien und das damit verbundene Stressempfinden zu beenden (Del Vecchio et al. 2009). So führten Videoaufnahmen (Frodi und Lamb 1980) und Tonbandaufnahmen (Crowe und Zeskind 1992, Riem et al. 2011) von schreienden Kindern bei Erwachsenen zu einem Anstieg der HR. Die hier untersuchten Patientinnen scheinen demnach ein abweichendes Reaktionsverhalten der HR auf Babyschreien aufzuweisen. Casanova et al. (1994) fanden sowohl bei Müttern mit als auch ohne erlebte körperliche Misshandlungen in der Kindheit keine Veränderung der HR während Babyschreien. Dies würde zu dem hier beobachteten Reaktionsmuster passen. Während Videoaufnahmen eines schreienden Babys bei Müttern ohne 
Missbrauchserfahrung eine erwartete sympathische Aktivierung in Form eines Anstiegs der Hautleitfähigkeit hervorriefen, blieb dieser bei Müttern mit erlebten körperlichen Misshandlungen in der Kindheit aus. Mütter mit kindlichen Misshandlungserfahrungen scheinen weniger sensitiv auf einen negativen emotionalen Status des Kindes anzusprechen (Casanova et al. 1994). Diese Schlussfolgerung könnte auch auf die untersuchten Frauen mit PTBS übertragen werden, denen in der Mehrzahl körperlicher bzw. sexueller Missbrauch in der Kindheit widerfahren und dieser als ursächlich für ihre PTBS-Diagnose anzusehen ist. Eine sehr stark ausgeprägte Reagibilität auf aversive kindbezogene Stimuli (Babyschreien) in Form eines höheren Anstiegs von HR und Hautleitwert scheint mit einem harschen Antwortverhalten gegenüber dem Kind verbunden zu sein (Crowe und Zeskind 1992) und wurde auch für physisch misshandelnde Mütter nachgewiesen (Frodi und Lamb 1980). Einige Studien fanden einen höheren HR-Anstieg bei Männern im Vergleich zu Frauen während Babyschreien (Brewster et al. 1998, Out et al. 2010). In einer aktuellen Studie von Tkaczyszyn et al. (2012) zum Einfluss von Babyschreien auf hämodynamische Parameter bei jungen gesunden Probanden zeigte sich keine Veränderung der HR im Vergleich zur Baseline. Der CI nahm geringfügig, jedoch nicht signifikant zu (Tkaczyszyn et al. 2012). In neueren Studien wird eine genetische Komponente als wichtige Determinante für die HR-Reaktivität auf Babyschreien diskutiert (Out et al. 2010, Riem et al. 2011). So wurde ein spezifischer Genotyp des Oxytocin Rezeptors (OXTR GG) identifiziert, der mit einer sensibleren Kindererziehung vergesellschaftet ist. Mütter mit diesem Genotyp zeigten eine stärker ausgeprägte physiologische Reaktivität auf wiederholtes Babyschreien (HR-Anstieg), außer wenn sie zusätzlich depressive Symptome aufwiesen (Riem et al. 2011). Mütterliche Sensibilität könnte demnach mit stärkerer physiologischer Reaktivität auf Babyschreien assoziiert sein, die wiederum vom Genotyp des Oxytocin Rezeptors beeinflusst wird. Oxytocin wirkt beruhigend auf das Panik- bzw. Bindungssystem, welches durch Distress Vocalisations, zu denen Babyschreien zählt, aktiviert wird (Panksepp et al. 1991). Feldman et al. (2007) wiesen eine Korrelation des Oxytocin-Plasmaspiegels bei Müttern und deren Kindern mit dem Ausmaß von sozialem Bindungsverhalten nach. Oxytocin spielt möglicherweise eine wichtige Rolle bei der Interaktion von Mutter und Kind und ist positiv assoziiert mit sensiblerer Kindererziehung (Feldman et al. 2007). Es sollte in künftigen Studien überprüft werden, ob die eingeschränkte HRReaktion der PTBS-Patientinnen auch mit Veränderungen des Oxytocin-Spiegels zusammenhängt. Der von Riem et al. (2011) beobachtete negative Effekt depressiver Symptome auf die physiologische Reaktivität auf Babyschreien unterstreicht den oft nachgewiesenen negativen Einfluss mütterlicher Depression auf die Mutter-Kind-Interaktion und Sensibilität auf kindliche Distress-Signale (Donovan et al. 1998). Folglich ist auch nicht auszuschließen, dass die hohe Anzahl an komorbiden depressiven Störungen im untersuchten PTBS-Patientinnenkollektiv die physiologische Reaktivität (HR-Anstieg) auf das Babyschreien reduzierte. Ein weiterer Erklärungsansatz ist das während des Babyschreiens im Vergleich zum Rechentest häufiger beobachtete Numbing-Verhalten der Patientinnen. Für die traumatisierten Frauen stellt das Babyschreien ggf. einen Trigger für dissoziative Verhaltensweisen 
dar. Dissoziative Symptome könnten zu einem geringeren Bewusstmachen des aversiven kindbezogenen Stimulus (Babyschreien) führen und somit die autonome Reaktivität in Form von eingeschränkter HR-Reaktivität beeinflussen (Lanius et al. 2006). Reagiert eine Mutter mit Dissoziation statt Zuwendung auf Distress Vocalisations ihres Kindes, kann dies fatale Folgen für die Entwicklung des Bindungssystems haben und ebenfalls zu dysfunktionalen Stressregulationsmechanismen mit dissoziativen Zügen beim Kind führen (Sachsse 2003).

Zusammenfassend scheinen PTBS-Patientinnen auf einen emotionalen Stressor (Babyschreien) mit einer geringeren Aktivierung von $\mathrm{HR}$ und $\mathrm{CI}$ im Vergleich zu einem kognitiven Stressor (Kopfrechentest) zu reagieren. Mögliche Erklärungsversuche reichen von Einfluss stärker ausgeprägter dissoziativer oder depressiver Symptome bis hin zu einer gestörten Anpassungsfähigkeit des Bindungssystems. Oder aber die beiden gewählten Stresstests aktivieren generell unterschiedliche Stressverarbeitungssysteme.

Das Babyschreien führte wie erwartet zu einem stärkeren Anstieg des TPRI als der Rechentest, welcher auch im Paarvergleich statistische Signifikanz erreichte. Ein emotionaler Stressor ist demnach in der Lage, eine vorwiegend alpha-adrenerge Aktivierung in Form eines Anstiegs des Gefäßwiderstandes hervorzurufen. Der Anstieg des sBP fiel jedoch während des Babyschreiens weniger stark aus, sodass der Unterschied zum Ausgangswert im Gegensatz zum Rechnen nicht signifikant war. Die vorliegenden Ergebnisse wären möglicherweise anders ausgefallen, wenn der Stressor „Babyschreien“ vor dem Stressor „Rechentest“ eingesetzt worden wäre. Frodi und Lamb (1980) hingegen hatten einen Anstieg des dBP bei gesunden Müttern während Babyschreien beobachtet, welcher besonders stark bei misshandelnden Müttern ausgeprägt war. Tkaczyszyn et al. (2012) fanden interessanterweise eine Abnahme des TPRI unter Babyschreien bei gesunden, jungen Probanden sowie eine geringe Abnahme des diastolischen Blutdrucks.

Der bei den PTBS-Patientinnen gering ausgeprägte bzw. fehlende Anstieg des Blutdrucks während des Babyschreiens kann zum einen auf die ausgebliebene Erholung nach dem vorangegangenen Stresstest (Rechnen) zurückzuführen sein. Andererseits könnte auch eine eingeschränkte Regulationsfähigkeit des Blutdrucks vermutet werden, wie sie von einigen Forschern für die PTBS postuliert wurde (Peckerman et al. 2003). Während der Rechentest als kognitiver Stressor in der vorliegenden Studie auf eine eingeschränkte Reaktivität des peripheren Gefäßwiderstandes hindeutet, scheint das Babyschreien als emotionaler Stressfaktor in erster Linie die Blutdruckregulation zu beeinflussen. Trauma-neutrale Stimuli führten auch in vorangegangenen Studien bei PTBS-Patienten im Vergleich zu Kontrollprobanden zu einer geringer ausgeprägten Blutdrucksteigerung (Peckerman et al. 2003, Keane et al. 1998, Blanchard et al. 1996), was zu den vorliegenden Ergebnissen während des Babyschreiens passt. Traumaassoziierte Stimuli hingegen lösten eine stärkere Blutdruckreaktivität bei den PTBS-Patienten aus (Keane et al. 1998, Pole 2007). In Widerspruch zu diesen Ergebnissen fanden Keary et al. (2009) bei Frauen mit und ohne PTBS einen vergleichbaren Blutdruckanstieg unter 
Stressanforderung. Der von Peckerman et al. (2003) bei Kriegsveteranen mit chronischer Fatigue und komorbider PTBS beobachtete eingeschränkte Anstieg des TPR während verschiedener Stresstests, konnte für das Babyschreien nicht bestätigt werden.

Der Rechentest und das Babyschreien scheinen unterschiedliche Defizite in der Regulation von Blutdruck und Gefäßwiderstand bei PTBS aufzudecken. Während beim Rechnen der TPRI nicht signifikant ansteigt, bleibt während des Babyschreiens ein erneuter Blutdruckanstieg aus, obwohl der TPRI adäquat erhöht wird. Möglicherweise liegt hier eine fehlerhafte Integrität höherer Hirnstrukturen zur Blutdruckregulation bei PTBS zugrunde (Peckerman et al. 2003).

\subsubsection{Autonome Parameter während Babyschreien}

Die SDNN sinkt erwartungsgemäß während des Babyschreiens im Vergleich zum Ausgangswert (Ruhe 1) ab. Dieser Unterschied ist jedoch nicht signifikant. Das Absinken der SDNN während des Babyschreiens spiegelt eine psychophysiologische Aktivierung der Patientinnen mit SympathikusAnstieg bzw. Vagusabfall durch diesen emotionalen Stresstest wider (Task Force 1996), wie sie auch während des Rechentests stattfand. Auch Tkaczyszyn et al. (2012) sahen bei gesunden, jungen Probanden eine Abnahme der SDNN als Zeichen eines vagalen Rückzugs unter Babyschreien. Die Anpassungsfähigkeit der RR-Intervalle scheint jedoch bei den hier untersuchten PTBS-Patientinnen eingeschränkt zu sein, denn während beider Stresstests waren die Paarvergleiche zur Ruhe 1 bzw. zwischen Rechnen und Babyschreien nicht signifikant.

Für die weiteren autonomen Parameter zeigt sich interessanterweise ein unterschiedliches bzw. gegensätzliches Reaktionsverhalten während des Babyschreiens im Vergleich zum Rechentest. Die vagal modulierte HF-HRV verändert sich während des Babyschreiens kaum in Bezug zum Ausgangswert in Ruhe 1. Die LF-HRV, als vorwiegend sympathisch modulierte HRV-Komponente, nahm während des Babyschreiens unerwarteterweise ab. Hier zeigt sich ein signifikanter, gegensätzlicher Effekt der beiden Stressoren „Rechnen“ und „Babyschreien“, denn der Rechentest hatte zu einer sympathischen Aktivierung (signifikanter Anstieg der LF-HRV) geführt. Auch auf die Sensitivität der Barorezeptoren (BRS) haben die beiden Stresstests signifikant unterschiedliche Auswirkungen. Während der Rechentest im Vergleich zur Ruhephase 1 graphisch zu einer Abnahme der BRS führte, resultierte das Babyschreien in einer Zunahme der BRS. Das Babyschreien löst bei den PTBS-Patienten demnach eher eine parasympathische Aktivierung aus. Der unterschiedliche Effekt der beiden Stresstests auf die BRS erwies sich im Paarvergleich als signifikant.Das Babyschreien entspricht nicht dem typischen Stressreaktivitätsmuster mit Vagusabfall und Zunahme der sympathischen Aktivität und ist daher nicht mit klassischen Stresstests wie Rechenaufgaben oder traumaassoziierten Stimuli gleichzusetzen. In der Studie von Tkaczyszyn et al. (2012) zeigte sich eine signifikante Abnahme der HF-HRV während des Babyschreiens. Diese war bei Frauen sogar stärker ausgeprägt als bei Männern. Ein schreiendes Baby verursacht demnach bei gesunden Frauen einen deutlicheren Rückzugs der vagalen Aktivität und verdeutlicht die spezifische Rolle des Stimulus Babyschreien, kindlichen Distress zu signalisieren und bei der Mutter bzw. Betreuungsperson eine 
Reaktion zu erzwingen. Bei den hier untersuchten PTBS-Patientinnen scheint diese natürliche Reaktion einer Vagusabnahme unter Babyschreien nicht auslösbar bzw. eingeschränkt zu sein. Stattdessen spiegeln die autonomen Parameter bei ihnen ein Überwiegen des Parasympathikus wider. Im Bereich der vorwiegend alpha-adrenergen Modulation konnte jedoch für den TPRI eine Aktivierung gesehen werden. Der Anstieg der BRS sowie der Abfall der LF-HRVpassenzur beobachteten leichten Abnahme der HR während des Babyschreiens. Als potentiell steigernder Einflussfaktor auf die BRS muss der vermehrte Einsatz von kompensierenden Atemübungen (vertieftes „Herunteratmen“) durch die Patientinnen während des Babyschreiens in Betracht gezogen werden (Bernardi et al. 2002).

Es zeigt sich also während des Babyschreiens für die autonomen Parameter eine Aktivitätssteigerung des Parasympathikus (BRS-Anstieg) und eine abnehmende LF-HRV und somit in diesen Parametern ein signifikant gegensätzliches Reaktivitätsmuster im Vergleich zum Rechentest, während der TPRI während des Babyschreiens anders als in der gesunden Stichprobe von Tkaczyszyn et al. (2012) in unserer Patientinnengruppe signifikant stärker anstieg als während des Rechentests. Jedoch sind abgesehen von der TPRI-Steigerung die Veränderungen aller autonomen Parameter während des Babyschreiens im Vergleich zur Baseline nicht signifikant, sodass die bereits für den Rechentest diskutierte eingeschränkte vagale Modulationsfähigkeit auch während des Babyschreiens zum Tragen kommt. Die Dissoziation gilt als parasympathikotone, hypometabolische Regulationsstrategie, die in Stresssituationen einem Ausklinken dient (Lanius et al 2006). Das während des Babyschreiens bei den Patientinnen vermehrt beobachtete Numbing-Verhalten könnte somit eine mögliche Erklärung für den im Vergleich zum Rechentest gesteigerten Vagotonus bieten. Unerwarteterweise zeigte auch die in einer parallelen Arbeit von G. Bornschein auf gleiche Weise untersuchte gesunde Kontrollgruppe während des Babyschreiens einen deutlichen, sogar viel stärker ausgeprägten Anstieg des Vagotonus in Form einer erhöhten HF-HRV und BRS (mündliche Mitteilung vom 23.10.2012). Vor diesem Hintergrund kann die Reaktion auf das Babyschreien als eine Aktivierung des parasympathisch modulierten Bindungssystems verstanden werden, die bei den PTBS-Patientinnen im Vergleich zu gesunden Frauen eingeschränkt zu sein scheint (kein Absinken der HF-HRV, keine signifikanten Veränderungen der autonomen Parameter im Vergleich zu den Ausgangswerten).

Schlussendlich zeigte sich während des Babyschreiens zwar ein ausgeprägtes subjektives Stressempfinden und eine vermehrte vegetative Begleitsymptomatik, aber in Bezug auf die Herzkreislauf-Parameter konnte keine klassische Stressreaktion im Sinne einer Aktivierung des Furchtsystems- wie sie ansatzweise während des Kopfrechentests gesehen wurde - nachgewiesen werden. Es ergaben sich signifikant unterschiedliche Paarvergleiche von Rechnen und Babyschreien für die Parameter HR, CI, TPRI, LF-HRV und BRS. Überraschenderweise führte das Babyschreien zu einem Absinken von HR und CI bzw. zu keinem signifikanten Unterschied zur Ruhephase 1und entsprach folglich nicht dem oft beobachteten Arousal auf Babyschreien mit HR-Zunahme bei 
gesunden Erwachsenen (u.a. Frodi und Lamb 1980). Die zur HR-Abnahme passende parasympathische Dominanz (Zunahme der BRS, Abnahme der LF-HRV) könnte einerseits auf beobachtete dissoziative Copingmechanismen zurückzuführen sein. Die Patientinnen zeigten nämlich ein vermehrtes Numbing-Verhalten während des Babyschreiens im Vergleich zum Rechentest. Andererseits zeigte eine gesunde Kontrollgruppe ebenfalls eine Zunahme des Vagotonus (HF-HRV und BRS), die sogar deutlich stärker ausgeprägt war und eventuell eine Aktivierung des parasympathisch modulierten Bindungssystems widerspiegelt. Diesbezüglich wäre ein fehlender HFHRV Anstieg der PTBS-Patientinnen und eine insignifikante Zunahme der BRS bzw. Abnahme der LF-HRV während des Babyschreiens als eine ineffiziente Regulation des Bindungssystems bei (k)PTBS bzw. BPS zu interpretieren. Insgesamt kommt auch hier die eingeschränkte vagale Regulationsfähigkeit bei PTBS heraus. Zudem wurde eine eingeschränkte Blutdrucksteigerung beobachtet, während der TPRI signifikant und im Vergleich zum Rechentest sogar verstärkt anstieg.

\subsubsection{Entspannungsmusik}

Zahlreiche psychophysiologische Studien an Gesunden haben gezeigt, dass Musik ein sehr potenter Stimulus ist, um beim Hörer eine Vielzahl von Emotionen hervorzurufen, die mit entsprechenden physiologischen Veränderungen einhergehen (u.a. Krumhansl 1997, Khalfa et al. 2008, Iwanaga et al. 2005, Orini et al. 2010). Sinkende Cortisolwerte durch Musikapplikation deuten auf eine Entspannung hin (Baule 2003). In der vorliegenden Studie wurde deshalb zum Abschluss der Stressbelastungsuntersuchung intuitiv die Mondscheinsonate von Beethoven gewählt, um bei den Patientinnen eine Entspannung zu erzielen. Sowohl das subjektive Stressempfinden als auch einige physiologische Parameter sanken jedoch während der Entspannungsmusik nicht wie erwartet auf das Ausgangsniveau zu Beginn der Untersuchung ab, sodass nicht bei allen Patientinnen die gewünschte Entspannung eingetreten zu sein scheint. Wie erwartet sanken HR, CI und LF-HRV während der beruhigenden Musik ab und HF-HRV und BRS stiegen an. Im Bereich der vorwiegend alphaadrenergen Parameter wurde jedoch ein Anstieg des TPRI gesehen, was auf ein Arousal hindeutet, und auch die Blutdruckwerte sanken nicht adäquat ab. Auch das Absinken der SDNN spricht nicht für eine vollkommene physiologische Entspannung durch die Mondscheinsonate.

Die Ergebnisse der subjektiven Analogskala zum Stressempfinden mit einem Mittelwert von 4,2 und einer breiten Streuung der Werte zeigen, dass es entgegen den Erwartungen große interindividuelle Unterschiede bei den hervorgerufenen Emotionen während des Musikhörens gab. $35 \%$ der Patientinnen empfanden die gewählte Sonate sogar als unangenehm bzw. emotional aufwühlend und nicht entspannend, sodass sechs Patientinnen diesen Teil der Untersuchung vorzeitig abbrechen mussten. Dass das gleiche Musikstück so verschieden wahrgenommen und emotional bewertet wurde, ist vermutlich auf unterschiedliche Interpretationen und frühere Erfahrungen bzw. Erinnerungen in Verbindung mit dieser oder ähnlicher Musik zurückzuführen. In einer Untersuchung von G. Baule (2003) zur Wirkung einer Klangliege auf psychologische und physiologische Parameter zeigte sich insbesondere bei Frauen eine stärkere subjektive Aktivierung trotz Beruhigung des Vegetativums 
(keine Steigerung der sympathischen Parameter). Es kann also davon ausgegangen werden, dass das subjektive Stressempfinden nicht zwangsläufig Rückschlüsse auf die physiologische Erholung durch Musik zulässt. Ebenfalls unerwartet waren die beobachteten emotionalen bzw. vegetativen Begleiterscheinungen (schweres Atmen, Erregungszustände, Weinen, Nutzen von Copingmechanismen, Numbing) während der beruhigenden Musik, die zwar weniger stark ausgeprägt im Vergleich zum Babyschreien waren, aber dennoch auf eine innere Erregtheit der Patientinnen hinweisen. Möglicherweise liegt in diesem ähnlichen Verhaltensmuster während Musik und Babyschreien auch eine Erklärung für die tendenziell vergleichbaren physiologischen Veränderungen im Rahmen dieser zwei Abschnitte der Untersuchung.

\subsubsection{Hämodynamische Parameter während Musik}

Wie erwartet führte die Entspannungsmusik zu einem Absinken von HR und CI sogar unter Ausgangsniveau (Ruhe 1). Für die HR ergab sich dadurch sogar ein signifikanter Unterschied zur Ruhephase 1. Dies entspricht Befunden zahlreicher Studien, die ein Absinken der HR durch beruhigende Musik bei gesunden Probanden nachwiesen (Krumhansl 1997, Knight und Rickard 2001, Bernardi et al. 2006). Krumhansl (1997) fand einen HR-senkenden Effekt durch musikalische Stimuli generell. Sowohl traurige, angsteinflößende als auch fröhliche Musikausschnitte führten bei gesunden Probanden zu einer Abnahme der HR, wobei diese bei dem traurigen Stimulus am ausgeprägtesten war. Diese und andere Studien machen deutlich, dass die emotionale Bewertung während des Musikhörens einen entscheidenden Einfluss auf die HR-Reaktivität auszuüben scheint. Entgegen den Beobachtungen von Krumhansl (1997) gibt es auch Studien, die einen HR-Anstieg durch musikalische Stimuli beobachteten, wobei die Ergebnisse in Bezug auf die emotionale Bewertung inkonsistent sind. In einigen Studien führte positive Musik zu einem stärkeren HR-Anstieg (Orini et al. 2010, Iwanaga und Moroki 1999). Andere Studien fanden wiederum keinen Unterschied der HR-Reaktivität im Vergleich von trauriger und fröhlicher (Khalfa et al. 2008) bzw. erregender und beruhigender Musik (Iwanaga und Tsukamoto 1997). In einer Studie von Bernardi et al. (2006) war der HR-Anstieg abhängig vom Tempo des Musikstückes, je schneller desto höher die HR.

In Bezug auf die Parameter HR und CI scheint die Mondschein-Sonate von Beethoven hier wie erwartet einen entspannenden Einfluss auf das Vegetativum der PTBS-Patientinnen auszuüben.

Interessanterweise erreichte der TPRI seinen maximalen Phasenmittelwert während der Entspannungsmusik, sodass sich ein signifikanter Unterschied zum Ausgangswert (Ruhe 1) ergab. Dies könnte auf erregende Eigenschaften der Musik für die PTBS-Patientinnen hinweisen. Der sBP hingegen fiel während der Sonate leicht ab, sodass kein signifikanter Unterschied zum Baseline-Wert bestand. Diastolischer und mittlerer arterieller Blutdruck blieben auf gleichem Niveau wie während der vorangegangenen Ruhephase, in der der Blutdruck nach dem Babyschreien nicht wie erwartet abgesunken war. Der Einfluss von Musik auf den arteriellen Blutdruck wurde in einigen psychophysiologischen Studien - jedoch nicht an PTBS-Kollektiven - untersucht, wobei die 
Ergebnisse sehr uneinheitlich sind. Krumhansl (1997) postulierte, dass unterschiedliche Emotionen mit spezifischen physiologischen Reaktionsmustern assoziiert sind. Er teste drei musikalisch induzierte Emotionen (traurig, ängstlich und fröhlich), die alle zu einem Anstieg der drei BlutdruckMessgrößen sBP, dBP und mBP bei gesunden Probanden führten. Dabei war der Blutdruckanstieg am stärksten ausgeprägt während des traurigen Musikausschnittes. Auch andere Studien fanden einen Blutdruckanstieg durch Musik, besonders stark ausgeprägt bei schnellem Tempo (Bernardi et al. 2006) und bei fröhlichem (Khalfa et al. 2008) bzw. erregendem Charakter der Musik (Iwanaga und Moroki 1999). Dieser Blutdruck steigernde Effekt durch Musik wurde in der vorliegenden Studie bei den PTBS-Patientinnen nicht bestätigt. In Ansätzen konnte bezogen auf den sBP ein mildernder Einfluss der Entspannungsmusik auf den zuvor Stress-induziert gesteigerten Blutdruck gesehen werden. Dies entspricht annähernd dem in einigen Studien beobachteten Blutdruck senkenden und angstlösenden Effekt von Musik im präoperativem Setting (Allen et al. 2001) bzw. in stressvollen Situationen (Knight und Rickard 2001). Jedoch wurde der Ausgangswert des systolischen Blutdrucks während der Entspannungsmusik nicht erreicht und der Gefäßwiderstand stieg sogar weiter an, sodass davon ausgegangen werden muss, dass das gewählte Musikstück nicht den erwünschten Entspannungscharakter für die Patientinnen entfaltete. Insgesamt ist die Reaktivität des TPRI und des arteriellen Blutdrucks während der Musik vergleichbar mit dem Reaktionsmuster während des Babyschreiens und demnach eher einem Arousal mit Stresserleben zuzuordnen. Lediglich für den sBP könnten Entspannungstendenzen eingeräumt werden, da kein signifikanter Unterschied zur Baseline bestand. Ungewöhnlich ist das entkoppelte Antwortverhalten des TPRI und des arteriellen Blutdrucks, normalerweise wäre ein gleichgesinntes Änderungsmuster zu erwarten gewesen. Diese Entkopplung war bereits während der Stresstests „Rechnen“ und „Babyschreien“ aufgefallen und dort als mögliche zentral fehlgesteuerte Blutdruckregulationsfähigkeit interpretiert worden (Peckerman et al. 2003).

\subsubsection{Autonome Parameter während Musik}

Im Bereich der autonomen Parameter zeigte sich lediglich für die BRS ein signifikanter Paarvergleich von Baseline und Musik, was bereits zeigt, dass sich die Werte der übrigen Indizes während der Entspannungsmusik an das Ausgangsniveau annäherten. Die HF-HRV stieg während der Entspannungsmusik erwartungsgemäß an und erreichte sogar den maximalen Phasenmittelwert. Die entspannende Musik erzielte analog dazu ein Absinken der LF-HRV auf Ausgangsniveau (Ruhe 1). Dies entspricht dem klassischen Bild von Erholung mit Sympathikus-Rückzug und vagaler Dominanz. Passend zu diesem Bild, konnte auch für die BRS eine Zunahme verzeichnet werden, die sogar den Ausgangswert (Ruhe 1) signifikant überstieg. Die SDNN fiel während der Entspannungsmusik unerwarteterweise unter den Ausgangswert in Ruhe $1 \mathrm{ab}$, der Paarvergleich war jedoch nicht signifikant. Betrachtet man die autonomen Parameter aus dem Frequenzbereich, so ist dieser Abfall am ehesten auf ein Absinken der sympathischen Aktivität zurückzuführen. Da jedoch zusätzlich der Vagotonus gestiegen ist (Zunahme von HF-HRV und BRS), wäre eine gleichbleibende SDNN bzw. ein Anstieg derselben zu erwarten gewesen. Das Absinken der SDNN könnte demnach einen 
versteckten Hinweis auf eine fehlende Entspannung bei manchen Patientinnen geben. In anderen Studien hatte traurige (Krumhansl 1997) bzw. langsame (Bernardi et al. 2006) Musik zu einer Zunahme der RR-Intervalle geführt.Van der Zwaag et al. (2011) untersuchten den Einfluss von langsamer und schneller Musik auf HRV-Parameter aus dem Zeitbereich (RR-Intervalle, RMSSD). Ein schnelleres Tempo der Musik führte zu einer Abnahme der HRV und gleichzeitig zu einer zunehmenden subjektiven Anspannung bei den gesunden Teilnehmern. Dies deutet darauf hin, dass erregende Musik ein Absinken der parasympathischen Aktivität herbeiführen kann. Langsame Musik hat hingegen einen positiven Effekt auf die HRV (van der Zwaag et al. 2011). Einige Studien beschrieben den Einfluss von Musik und musikalisch induzierten Emotionen auf HRV-Parameter aus dem Frequenzbereich. In einer Studie von Iwanaga et al. (2005) stiegen LF-HRV und LF/HF Ratio, während die Teilnehmer Musik (beruhigende und erregende) hörten bzw. sanken während Ruhephasen ohne Musik. Die HF-HRV war während der beruhigenden Musik höher als während der erregenden Musik, jedoch genauso hoch wie in Ruhephasen. Auch subjektiv induzierte die beruhigende Musik ebenso wie die Ruhephasen ein beruhigendes Gefühl. Es zeigt sich, dass erregende Musik die Aktivität des parasympathischen Nervensystems herabsetzt, während beruhigende Musik diese zu steigern vermag. Iwanaga et al. (2005) bezeichnen die HF-HRV als sensitiven Index zur Bestimmung der emotionalen Entspannung durch Musik. Diese Ergebnisse passen annähernd zu den hier beobachteten Veränderungen der HF-HRV. Die HRV-Parameter aus dem Frequenzbereich, die hier eine vagale Dominanz repräsentieren, stellen den entspannenden Charakter der gewählten Sonate heraus. Vergleichbare beruhigende Effekte durch Musik auf subjektiver und physiologischer Ebene wurden auch in anderen Studien gesehen. Lee et al. (2011) spielten Patienten, die auf eine Operation warteten, Instrumentalmusik vor. Durch die Musikapplikation sanken sowohl die Angaben der Patienten auf einer visuellen Analogskala für Angst als auch die LF-HRV und LF/HF-Ratio, als Indizes sympathischer Aktivität, im Vergleich zur Kontrollgruppe ohne Musik. Iwanaga und Tsukamoto (1997) berichteten, dass beruhigende Musik einen größeren Anstieg der HF-HRV induziert als erregende Musik. Bernardi et al. (2006) untersuchten neben zahlreichen kardiovaskulären Parametern und der HRV auch die BRS. Passives Musikhören führte zu einem proportionalen Anstieg der LF/HF Ratio abhängig vom Tempo der Musik, was eine sympathische Aktivierung widerspiegelt. Die BRS sank tendenziell mit ansteigendem Tempo des Musikstïckes ab. Musik hat auf der einen Seite einen erregenden Effekt, in erster Linie abhängig vom Tempo. Auf der anderen Seite kann langsame oder meditative Musik zu einer Entspannung und zu einer Zunahme der BRS führen (Bernardi et al. 2006). Diese Ergebnisse entsprechen den Beobachtungen der vorliegenden Studie. Die erhöhte BRS steht für eine Zunahme des Vagotonus (La Rovere et al. 1995) und unterstreicht ebenso wie die HRVParameter aus dem Frequenzbereich den entspannenden Einfluss der Mondscheinsonate auf die PTBSPatientinnen. 
Zusammenfassend offenbarte die emotionale Bewertung der Entspannungsmusik große interindividuelle Unterschiede, sodass die Angaben auf der Analogskala zum subjektiven Stressempfinden nicht das Ausgangsniveau $\mathrm{zu}$ Beginn der Untersuchung erreichten. Die Veränderungen der beta-adrenergen Parameter HR und CI sowie der autonomen Parameter HF-HRV, LF-HRV und BRS spiegeln einen beruhigenden Effekt der Musik wider. Der starke Anstieg des TPRI. die eingeschränkte Abnahme des arteriellen Blutdrucks sowie die fehlende Zunahme der SDNN und das Auftreten vegetativer bzw. emotionaler Begleitsymptome deuten auf erregende Eigenschaften der Sonate für einige Patientinnen hin. Zahlreiche Studien haben Angst lösende und entspannende Eigenschaften von Musik beispielsweise im prä-/perioperativen Setting (Allen et al. 2001, Lee et al. 2011) herausgestellt. Auch für die PTBS gibt es erste Ansätze, Musik therapeutisch zu nutzen (Behrens 2008, Carr et al. 2012). Musiktherapie dient der Reintegration von Sinneseindrücken und Emotionen sowie der Entwicklung emotionaler Copingstrategien. Ein physiologisches Hyperarousal, nicht integrierte fragmentierte Erinnerungen, welche durch Trigger zu einem ständigen Wiedererleben des Traumas führen, sowie das Unvermögen, aufgrund einer reduzierten linkshemisphärischen Aktivität über das Trauma zu sprechen, prägen das klinische Bild der PTBS (Sachsse et al. 2009). Musik kann dazu beitragen, Emotionen und Reaktionen zu kontrollieren und zu modulieren und langfristig die traumatischen Erfahrungen zu integrieren. Behrens (2008) erzielte mit kriegstraumatisierten Kindern aus Palästina (West Bank), die eine hohe Inzidenz von PTBS, Angststörungen, Depression und Verhaltensauffälligkeiten zeigten, erste musiktherapeutische Behandlungserfolge. In einer aktuellen Studie von Carr et al. (2012) konnte eine GruppenMusiktherapie die Schwere der PTBS- sowie der depressiven Symptomatik bei traumatisierten Patienten, die nicht auf eine kognitive Verhaltenstherapie ansprachen, signifikant senken. Die PTBSPatienten empfanden die Musik als sehr hilfreich. Auch wenn die gewählte Musik der vorliegenden Studie (Mondscheinsonate von Beethoven) nicht für alle Patientinnen die gewünschte Beruhigung herbeiführte, so stellten doch der Großteil der physiologischen Parameter eine innere Entspannung und damit auch den Nutzen von musikalischen Stimuli zur Herabregulation eines Stress-induzierten Arousal bei PTBS-Patientinnen heraus. Diese Ergebnisse sind vielversprechend für den zukünftigen Einsatz von Musik im Rahmen der PTBS-Therapie, jedoch sollten die Patientinnen die Musik evtl. nach eigenen Präferenzen wählen können.

\subsubsection{Rückbildungsfähigkeit nach Stressanforderung (Recovery)}

$\mathrm{Zu}$ den maladaptiven physiologischen Merkmalen der PTBS zählt neben veränderten RuheParametern sowie einer Hyperreaktivität auf stressvolle Stimuli auch eine gestörte Rückbildungsfähigkeit nach Stressanforderung (Recovery). Nach den Stresstests „Rechnen“ und „Babyschreien“ erfolgte deshalb jeweils eine fünfminütige Ruhephase, in der die Abnahme des Erregungsniveaus ermittelt und die Phasenmittelwerte der physiologischen Parameter mit den Ausgangswerten (Ruhe 1) verglichen wurden. Die meisten Parameter näherten sich nach den 
Stresstests im Mittel dem Ausgangsniveau zu Beginn der Untersuchung an, sodass die Paarvergleiche für den CI, die HR, die SDNN, die HF-HRV und die BRS nicht signifikant waren. Für den TPRI und die drei Blutdruck-Messgrößen (sBP, dBP und mBP) zeigten sich in der Ruhephase 2 nach dem Rechentest signifikant höhere Werte im Vergleich zur Ruhe 1. Die LF-HRV nahm in den Erholungsphasen (Ruhe 2 und 3) nach den zwei Stresstests „Rechnen“ und „Babyschreien“ jeweils signifikant höhere Werte als zu Beginn der Untersuchung (Ruhe 1) an. Somit scheint die Rückbildungsfähigkeit auf alpha-adrenerger und sympathischer Ebene bei den (k)PTBS-Patientinnen eingeschränkt.

Bei Gesunden fanden Mezzacappa et al. (2001) nach Stressanforderung u.a. durch einen Rechentest während der Erholungsphase eine niedrigere HR sowie eine höhere HRV (RMSSD) als zu Beginn des Experiments. Die Arbeitsgruppe schlussfolgerte, dass die kardiovaskuläre Erholung von Stress mit einer erhöhten vagalen Modulation (vagal rebound) assoziiert ist. Eine eingeschränkte vagale Erholung nach Stressanforderung ist zudem mit Standardrisikofaktoren für kardiovaskuläre Erkrankungen (männliches Geschlecht, positive Familienanamnese bezüglich kardiovaskulärer Erkrankungen) verknüpft. Auch Cole et al. (1999) stellten die These auf, dass ein eingeschränkter HRAbfall nach körperlicher Betätigung eine herabgesetzte vagale Reflexaktivität widerspiegelt und diese einen wichtigen Mortalitätsprädiktor darstellt. Die hier untersuchten PTBS-Patientinnen zeigten sowohl nach kognitiver (Rechentest) als auch emotionaler (Babyschreien) Stressanforderung lediglich eine geringe bzw. keine Zunahme des Vagotonus (HF-HRV und BRS) und die Werte waren nicht höher im Vergleich zum Ausgangsniveau in Ruhe. Die vagale Erholung scheint demnach eingeschränkt. Gleichzeitig sinkt die LF-HRV als vorwiegend sympathisch modulierter HRVParameter nach dem Rechentest kaum ab und steigt nach dem Babyschreien sogar an, sodass zusätzlich zur eingeschränkten vagalen Modulation von einer erhöhten sympathischen Aktivität in der Erholungsphase bei den PTBS-Patientinnen ausgegangen werden muss. Die Arbeitsgruppe Keary et al. (2009) beurteilte die Rückbildungsfähigkeit bei PTBS nach einem Rechentest und einem Trauma Recall. Insbesondere nach dem trauma-assoziierten Stressor erreichten die Werte für sBP, LF-HRV und HF-HRV nicht das Ausgangsniveau. In der vorliegenden Studie zeigte sich ein ähnliches Reaktionsmuster für den sBP und die LF-HRV jedoch nach dem trauma-neutralen Stressor „Rechnen“. Unterschiedlich war auch die Rückbildung der HR, welche in der Studie von Keary et al. (2009) nach dem Rechentest nicht auf Ruhelevel absank. Sack et al. (2004) fanden eine signifikant verlängerte Erholungs-Halbwertszeit (Half Recovery Time) für die HR bei PTBS-Patientinnen mit niedriger Ruhe-RSA im Vergleich zu PTBS-Patientinnen mit hoher Ruhe-RSA, was einer negativen Korrelation von Erholungs-Halbwertszeit und Baseline-RSA entspricht. Das bedeutet Patientinnen mit bereits in Ruhe eingeschränkter vagaler Kontrolle weisen auch eine verzögerte Rückbildungsfähigkeit der HR nach Stressanforderung auf.

Um die Rückbildungsfähigkeit nach dem emotionalen Stressor „Babyschreien“ besser beurteilen zu können, wurden neben den Phasenmittelwerten zusätzliche Messzeitpunkte einzelner Parameter 
betrachtet (siehe Kapitel 3.7.6, Seite 32). Es zeigte sich kein signifikanter Unterschied zwischen dem Ende des Babyschreiens und den drei Messzeitpunkten während der Ruhephase 3 für den CI, den sBP, den TPRI sowie die Root Mean Square of Successive Differences (RMSSD), was auf eine inadäquate Herabregulierung nach der Stressbelastung „Babyschreien“ hinweist. Der CI und der sBP stiegen zu den Messzeitpunkten nach dem Babyschreien sogar leicht an und die RMSSD, als ParasympathikusMarker, fiel überraschenderweise ab. Hierin zeigt sich deutlich, dass eine für die kardiovaskuläre Gesundheit entscheidende vagale Erholung (Mezzacappa et al. 2001, Cole et al. 1999) nach emotionaler Stressbelastung (Babyschreien) bei den PTBS-Patientinnen ausbleibt. Es sollte jedoch bedacht werden, dass die physiologische Erregung bei den PTBS-Patientinnen während des Babyschreiens in manchen Dimensionen sehr gering ausgeprägt war und daher eine schnelle Abnahme des Erregungsniveaus auch weniger zu erwarten gewesen ist als beispielsweise nach ErgometerBelastung oder Ärger-Expostition. Es könnte vermutet werden, dass das während des Babyschreiens vermehrt beobachtete Numbing-Verhalten die physiologische Stressreaktion währenddessen mildert, wie es für dissoziative Copingstrategien bekannt ist (Lanius et al. 2006), und deshalb die eigentliche Reaktion erst nach Ende des Stressors zum Vorschein tritt. Gutner et al. (2010) untersuchten die Rückbildungsfähigkeit verschiedener physiologischer Parameter nach trauma-assoziierten Monologen als möglichen prospektiven Prädiktor von PTSD-Symptomen. Die Erholung von HR und Hautwiderstand erwiesen sich als signifikante Prädiktoren für PTBS-typische Symptome des Wiedererlebens. Die HR-Erholung stellte sich zudem als signifikanter Prädiktor für verstärkte Numbing-Symptome heraus. Diese Ergebnisse zeigen, dass eine ausgedehnte Reaktivität bzw. eine verzögerte Rückbildungsfähigkeit nach Stressanforderung PTBS-typische Symptome sowohl aus dem Bereich Intrusion (Wiedererleben) als auch Vermeidungsverhalten (Numbing) begünstigen (Gutner et al. 2010). Pole stellte in seiner Metaanalyse zur psychophysiologischen Reagibilität bei PTBS neben einer veränderten Reaktivität auch insbesondere die gestörte Erholung bzw. Adaptationsfähigkeit an stressvolle Stimuli heraus (Pole 2007), welche in der vorliegenden Studie insbesondere nach dem Babyschreien bestätigt werden konnte.

\subsection{Patientinnen-Subgruppe ,hoch Borderline“}

Eine generelle emotionale Vulnerabilität (Anspannung, leicht zu triggernde affektive Reaktionen) wird als Hauptsymptom der BPS beschrieben (Linehan 1993), jedoch gibt es bisher nur wenige psychophysiologische Studien zu dieser These und die Ergebnisse sind zum Teil inkonsistent. Einige Studien konnten eine verstärkte emotionale Reaktion in Form eines physiologischen Hyperarousal auf Borderline-spezifische Stimuli nachweisen (Lobbestael und Arntz 2010, Elices et al. 2012, Limberg et al. 2011), andere Studien fanden ein physiologisches Hypoarousal bei BPS-Patienten (Herpertz et al. 1999, Schmahl et al. 2004). Der Tatsache einer hohen Komorbiditätsrate von PTBS und BPS (u.a. Sack et al. 2012) geschuldet, ist nicht eindeutig auszumachen, welche der beiden Pathologien die emotionale physiologische Reaktivität auf welche Art und Weise beeinflusst. Nur zwei Studien 
untersuchten unseres Wissens BPS- und PTBS-Patienten im Vergleich (Schmahl et al. 2004) bzw. den Einfluss einer komorbiden PTBS auf die physiologische Reagibilität bei Borderline-Patienten (Limberg et al. 2011). Um der Forschungslücke des Einflusses einer ausgeprägten BorderlineSymptomatik auf die Stressphysiologie von (k)PTBS-Patientinnen näher auf den Grund gehen zu können, wurde in der vorliegenden Studie eine Patientinnen-Untergruppe "hoch Borderline" anhand psychometrischer Merkmale gebildet und deren Reaktionsmuster während der Stressbelastungsuntersuchung mit den restlichen (k)PTBS-Patientinnen des Ausgangskollektivs ohne ausgeprägte Borderline-Symptomatik verglichen.

Der Subgruppe „hoch Borderline“ wurden anhand zuvor festgelegter Kriterien (BPI Cut-20 $\geq 10$, BSLSubskala Affektregulation >25 und BSL-Subskala Autoaggression >17,5) 16 Patientinnen zugeordnet (siehe Methodenteil Kapitel 3.8). Für die beiden Borderline-spezifischen Messinstrumente BPI und BSL zeigen sich erwartungsgemäß signifikant höhere Werte in der Subgruppe „hoch Borderline“ im Vergleich zum Rest des Ausgangskollektivs. Dem komplexen Symptomspektrum einer BPS entsprechend, weisen die (k)PTBS-Patientinnen mit starker Borderline-Symptomatik auch auf anderen Fragebögen erhöhte Werte im Vergleich zur Kontrollgruppe auf. Die Patientinnen der Subgruppe „hoch Borderline“ gaben stärker ausgeprägte depressive (BDI) und dissoziative Symptome (FDS, DES) sowie eine stärker erhöhte grundsätzliche psychische Belastung (SCL-90-R) an. Dieser psychometrische Befund der Subgruppe „hoch Borderline“ entspricht den Auswertungen einer aktuellen multizentrischen Studie zu Traumafolgestörungen bei BPS-Patienten (Sack et al.2012). Patienten, die sowohl die Kriterien für eine BPS als auch eine komplexe PTBS erfüllten, berichteten über eine signifikant stärker ausgeprägte depressive und dissoziative Symptomatik sowie somatoforme Körperbeschwerden und damit insgesamt über eine höhere Symptombelastung.

Während der TFM-Messung ergab sich kein Unterschied zwischen Sub- und Kontrollgruppe hinsichtlich der Angaben auf der Analogskala zum subjektiven Stressempfinden (SUD). Auch in der Studie von Schmahl et al. (2004) gaben PTBS- und BPS-Patienten vergleichbare Werte auf der SUD an.

Für die während der Stressbelastungsuntersuchung beobachtete vegetative Begleitsymptomatik wurde für die einzelnen Reaktionsformen schwere Atmung, Erregungszustand, Weinen, Einsatz von Copingmechanismen und Numbing-Verhalten kein signifikanter Gruppenunterschied gefunden. Eine Einteilung in Patientinnen mit $\leq 1$ vs. Patientinnen mit $>1$ vegetativen Reaktionsformen erbrachte keinen signifikanten Unterschied zwischen den Gruppen, auch wenn die hoch Borderline-Patientinnen prozentual gesehen häufiger mehrere vegetative Begleitsymptome vereint während einer emotionalen Stressbelastung (Babyschreien) aufwiesen. Die beobachteten vegetativen bzw. emotionalen Reaktionen passen zu Linehans Hypothese der generellen emotionalen Vulnerabilität mit affektiver Dysregulation bei Borderline-Patienten (Linehan 1993). Eine Korrelation zwischen der Anzahl vegetativer Reaktionen und den physiologischen Parametern war sowohl in der Subgruppe „hoch Borderline" als auch in der Vergleichsgruppe nachweisbar. 
Das physiologische Reagibilitätsmuster während der Stressbelastungsuntersuchung offenbarte signifikant höhere Blutdruckmesswerte ( $\mathrm{sBP}, \mathrm{dBP}$ und $\mathrm{mBP}$ ) sowie eine global fast signifikant herabgesetzte BRS bei den Patientinnen mit ausgeprägter Borderline-Symptomatik. Die restlichen hämodynamischen und autonomen Parameter zeigten keinen globalen signifikanten Unterschied zwischen Subgruppe und Kontrolle, jedoch konnte insbesondere während des Babyschreiens graphisch ein tendenzielles Hyperarousal in der Subgruppe „hoch Borderline“ beobachtet werden. Der durchgeführte Paarvergleich der beiden Stressoren (Rechnen vs. Babyschreien) ergab zwischen den Gruppen signifikant unterschiedliche Änderungen (Phasenmittelwert-Differenzen) für den sBP, den TPRI sowie fast signifikant für die BRS.

\subsubsection{Vergleich der hämodynamischen Parameter}

Im Verlauf der Stressbelastungsuntersuchung weisen die Patientinnen der Subgruppe „hoch Borderline“ graphisch insgesamt höhere HR-Werte auf (jedoch nicht signifikant). Der Rechentest führte in beiden Gruppen zu einer signifikanten Pulsbeschleunigung sowie Steigerung des CI. Während des Babyschreiens nahm die HR in der Kontrollgruppe ab, die Patientinnen mit ausgeprägter Borderline-Symptomatik zeigten hingegen einen HR-Anstieg. Die Phasenmittelwert-Differenzen des Paarvergleiches Rechnen vs. Babyschreien waren zwischen den Gruppen nicht signifikant unterschiedlich. In der dem Babyschreien anschließenden Erholungsphase (Ruhe 3) sinkt die HR in der Borderline-Gruppe nicht ab, was auf eine stärker eingeschränkte Rückbildungsfähigkeit nach Stressanforderung hinweisen könnte. Während der Entspannungsmusik sinken HR und CI als Zeichen einer physiologischen Herabregulation in beiden Gruppen ab.

Die Ergebnisse vorangegangener Studien zur HR-Reaktivität bei Patienten mit BPS auf emotionale Stimuli sind uneinheitlich. Auf neutrale bzw. nicht Borderline-bezogene Stimuli wurde oftmals ein Hypoarousal beobachtet (Schmahl et al. 2004, Elices et al. 2012), Borderline-spezifische Stressoren hingegen führten bei BPS-Patienten in verschiedenen Studien $\mathrm{zu}$ einer physiologischen Hyperreaktivität mit verstärktem HR-Anstieg (Lobbestael und Arntz 2010, Elices et al. 2012, Limberg et al. 2011). Herpertz et al. (1999) untersuchten als erste Arbeitsgruppe die Reaktivität von HR, Hautwiderstand und Schreckreaktion auf angenehme, neutrale und unangenehme Bilder bei Borderline-Patientinnen. Weder subjektive noch physiologische Daten erbrachten einen Hinweis, dass Borderline-Patientinnen eine gesteigerte affektive Reaktivität im Vergleich zu Gesunden aufweisen. Die Teilnehmerinnen mit BPS zeigten sogar eine herabgesetzte elektrodermale Reaktivität auf alle drei Stimuli, was ein physiologisches Hypoarousal widerspiegelt. Dieses überraschende Ergebnis steht in Widerspruch zur Linehans Hypothese einer biologisch verankerten affektiven Hyperreagibilität bei BPS (Linehan 1993). Herpertz et al. (1999) vermuten, dass chronischer Stress bei Personen mit BPS einen herab regulierenden Prozess mit nachfolgender autonomer Hyporeagibilität auslöst. Auch Schmahl et al. (2004) fanden keinen Unterschied der HR in Ruhe bei BPS-Patienten im Vergleich zu PTBS-Patienten ohne BPS und traumatisierten gesunden Kontrollprobanden. Die Borderline-Patienten zeigten auf Trauma-bezogene Stimuli im Vergleich zur PTBS-Gruppe eine eingeschränkte HR- 
Reaktivität. Zwar wurde in der hier vorliegenden Studie ebenfalls kein signifikanter Unterschied der HR zwischen der Subgruppe „hoch Borderline“ und der Vergleichsgruppe im gesamten Verlauf der Stressbelastungsuntersuchung gefunden, aber insbesondere während des Babyschreiens weist die hoch Borderline-Gruppe eine höhere HR auf und im Intragruppenvergleich zeigt sich in dieser Subgruppe kein signifikanter Unterschied zum Rechentest. In der Kontrollgruppe hingegen war die HR zwischen Rechnen und Babyschreien signifikant unterschiedlich. Eine von Herpertz et al. (1999) und Schmahl et al. (2004) beobachtete Hyporeaktivität der HR wurde hier während der beiden Stressoren „Rechnen“ und „Babyschreien“ nicht bestätigt. Das Reaktionsmuster der Subgruppe „hoch Borderline“ während des Babyschreiens ist ansatzweise vergleichbar mit einem vielfach gesehenen Hyperarousal (u.a. HR-Anstieg) auf Borderline-spezifische Stimuli. Im Rahmen einer Konfrontation mit Missbrauch-bezogenem Filmmaterial zeigten Borderline-Patienten eine Übererregbarkeit sowohl einiger psychophysiologischer Parameter (HR, Hautwiderstand und sBP) als auch für subjektive Angaben zu negativen Affekten sowie vermehrt maladaptive Verhaltensmuster (Lobbestael und Arntz 2010). Auch Elices et al. (2012) nutzten Emotionen-induzierende Filme u.a. auch mit Borderlinespezifischen Inhalten (sexueller Missbrauch, emotionale Abhängigkeit, Verstoßung usw.), um die emotionale Reaktivität von Borderline-Patienten anhand von physiologischen Parametern (HR und Hautwiderstand) besser beurteilen zu können. Der HR-Anstieg während Angst, Ärger und Trauer auslösender Filme war in der Borderline-Gruppe niedriger als in der Kontrollgruppe. Borderlinespezifische Inhalte hingegen lösten bei den BPS-Patienten ein Hyperarousal aus. Limberg et al. (2011) fanden bei Borderline-Patienten eine verstärkte Schreckreaktion sowie ein erhöhtes Arousal (HRAnstieg) in Bezug auf Borderline-spezifische Szenen (Verstoßung, Vernachlässigung). Standardisierte emotionale Skripte riefen keinen Unterschied in der emotionalen Modulation von Abwehrreflexen und autonomer Reaktion von BPS-Patienten und gesunden Kontrollen hervor. BPS-Patienten scheinen demnach nicht generell physiologisch hyperreaktiv auf emotionale Stimuli zu reagieren, sondern zeigen eine erhöhte emotionale Vulnerabilität, wenn Borderline-spezifische Schemata aktiviert werden (Limberg et al. 2011). Da auch das Babyschreien graphisch zu einem leichten Anstieg der HR ausschließlich in der Borderline-Gruppe führte, kann vermutet werden, dass dieser emotionale Stressor bei einigen Patientinnen als Borderline-spezifischer Trigger wirkt. Passend zum klinischen Merkmal der affektiven Dysregulation bei BPS geben diese Ergebnisse Hinweise auf eine gestörte Verarbeitung unangenehmer emotionaler Stimuli mit Hyperreagibilität bei Borderline-Patienten. Limberg et al. (2011) untersuchten weiterhin den Einfluss einer komorbiden PTBS auf die emotionale Reaktivität bei Borderline-Patienten. Lag zusätzlich eine PTBS vor, so zeigten die BPS-Patienten nicht nur ein Hyperarousal (HR-Anstieg) auf Borderline-spezifische Reize sondern auch auf andere aversive Bilder, was jedoch nicht bei Fehlen der PTBS-Diagnose beobachtet werden konnte. Die emotionale Reaktivität von BPS-Patienten mit komorbider PTBS scheint folglich gesteigert und auf verschiedene Stimuli erweitert zu sein (Limberg et al. 2011). Auf das hier untersuchte Patientinnenkollektiv mit (k)PTBS und die Subgruppe mit ausgeprägter Borderline-Symptomatik übertragen, kann spekuliert 
werden, dass eine zusätzlich zur Traumafolgestörung vorliegende BPS eine überspitzte physiologische Affektregulation beispielsweise auf alltägliche emotionale Stressoren wie Babyschreien begünstigt. Solche Trauma-Patientinnen mit ausgeprägter Borderline-Symptomatik bedürfen daher einer besonderen Behandlung mit Hinblick auf Affektstabilisierung, um physiologische Hyperarousal im Alltag besser kontrollieren zu können.

In der Subgruppe „hoch Borderline“ war sowohl der sBP als auch der TPRI im Mittel insgesamt deutlich erhöht im Vergleich zum Rest des Ausgangskollektivs. Für den sBP war dieser Gruppeneffekt signifikant. Während des Rechentests konnte in beiden Gruppen ein Blutdruckanstieg gesehen werden, während des Babyschreiens zeigen die beiden Untersuchungsgruppen graphisch unterschiedliche Reaktionsmuster. In der Borderline-Gruppe steigen sBP und TPRI während des emotionalen Stressors auf die jeweiligen maximalen Phasenmittelwerte an, was auf eine starke emotionale Erregung hindeutet. Die Kontrollfrauen ohne ausgeprägte Borderline-Symptomatik reagieren hingegen auf das Babyschreien mit einem Absinken von sBP und TPRI. Hieraus ergibt sich ein signifikanter Gruppenunterschied für die Phasenmittelwert-Differenzen des Paarvergleiches Rechnen vs. Babyschreien, was das unterschiedliche Reaktionsmuster während des Babyschreiens statistisch stützt. Die Arbeitsgruppe Schmahl et al. (2004) bestimmte u.a. Veränderungen des arteriellen Blutdrucks während neutraler sowie Trauma- und Borderline-bezogener Stimuli bei Patienten mit BPS oder PTBS und traumatisierten Kontrollen ohne PTBS. In Ruhe zeigte sich kein Unterschied des Blutdrucks zwischen BPS- und PTBS-Gruppe. Die PTBS-Patienten und die gesunden Teilnehmer mit Missbrauchserfahrung zeigten einen stärkeren Blutdruckanstieg als BPS-Patienten während Traumaassoziierter Skripte. Insgesamt zeigte sich bei den BPS-Patienten auf beide Stressoren eine eingeschränkte Reaktivität des Blutdrucks, was Schmahl et al. (2004) als Zeichen einer eingeschränkten physiologischen Reagibilität auf stressvolle Skripte bei BPS - eventuell auch bedingt durch höhere dissoziative Eigenschaften in der BPS-Gruppe - deuteten. Diese Ergebnisse konnten in der vorliegenden Studie nicht bestätigt werden, denn die hoch Borderline-Gruppe zeigte insbesondere auf den emotionalen Stressor „Babyschreien“ eine höhere Reaktivität (sBP-Anstieg) und hatte während der gesamten Stressbelastungsuntersuchung höhere Blutdruckwerte als die Vergleichsgruppe. Es muss auch berücksichtigt werden, dass die von Schmahl et al. (2004) untersuchten BorderlinePatienten teilweise auch eine komorbide PTBS hatten und es sich nicht um strikt distinkte Gruppen handelte und daher ein Einfluss posttraumatischer Zustände auf die physiologische Reagibilität nicht ausgeschlossen werden kann. In Widerspruch zu der von Schmahl et al. (2004) postulierten Hyporeagibilität bei BPS und im Einklang mit den hier vorgestellten Ergebnissen fanden Lobbestael und Arntz (2010) einen ausgeprägten Blutdruckanstieg bei Borderline-Patienten im Rahmen einer Konfrontation mit Missbrauch-bezogenem Filmmaterial. Borderline-spezifische Stimuli, aber anscheinend auch allgemeine emotionale Stimuli wie Babyschreien, scheinen demnach in der Lage zu 
sein bei (k)PTBS-Patientinnen mit ausgeprägter Borderline-Symptomatik ein physiologisches Hyperarousal mit Blutdruckanstieg auszulösen.

\subsubsection{Vergleich der autonomen Parameter}

Im Bereich der autonomen Parameter zeigt sich eine vagale Dysfunktion mit insgesamt fast signifikant erniedrigten Werten der BRS bei Vorliegen einer ausgeprägten Borderline-Symptomatik. Die HRV scheint in der Subgruppe „hoch Borderline“ eingeschränkter zu sein (insgesamt niedrigere Phasenmittelwerte für SDNN, HF- und LF-HRV), wobei kein signifikanter globaler Gruppeneffekt nachgewiesen werden konnte. Das Babyschreien löst in beiden Gruppen keine signifikanten Veränderungen im Vergleich zur Baseline für die HRV-Parameter aus, deutet jedoch in der Kontrollgruppe wie bereits für das Gesamtkollektiv beschrieben auf ein eher vagal dominiertes Reaktionsmuster hin (Anstieg der HF-HRV sowie Absinken der LF-HRV). Die BRS hingegen sinkt während des Babyschreiens in der Borderline-Gruppe ab, während diese in der Kontrollgruppe signifikant im Vergleich zum Ausgangswert in Ruhe 1 ansteigt. Hier könnte ein Hinweis auf einen stärker herabgesetzten Vaguseinfluss in der hoch Borderline-Subgruppe während des Babyschreiens liegen, denn auch der durchgeführte Paarvergleich der beiden Stressoren (Rechnen vs. Babyschreien) ergab fast signifikant unterschiedliche Änderungen zwischen den Gruppen ( $\mathrm{p}=0,051)$.

Bisher gibt es nur wenige Studien, die autonome Parameter bei Patienten mit BPS untersuchten und keine Studie nahm unseres Wissens eine Gegenüberstellung von (k)PTBS-Patientinnen mit und ohne ausgeprägter Borderline-Symptomatik vor, daher kann die Interpretation der hier vorgestellten Ergebnisse nur vorläufigen Charakter haben und muss in Folgestudien ergänzt und erweitert werden. In einer Studie von Kuo und Linehan (2009) war eine BPS-Diagnose assoziiert mit einer erniedrigten basalen RSA (reduzierter Vagotonus) im Vergleich zu gesunden Kontrollen, was als erhöhte biologische Vulnerabilität für eine emotionale Dysregulation gedeutet wurde. Des Weiteren hatten die Teilnehmer mit BPS eine erhöhte negative emotionale Erregtheit in Form einer erhöhten Hautleitfähigkeit in Ruhe und gaben ausgeprägte negative Empfindungen an. Die BPS-Gruppe zeigte jedoch keine erhöhte Reaktivität auf Emotionen-induzierende Filme im Vergleich zur gesunden Kontrollgruppe (Kuo und Linehan 2009). Die vorliegenden Ergebnisse bestätigen eine reduzierte vagale Aktivität auch bei Vorliegen einer ausgeprägten Borderline-Symptomatik bei (k)PTBSPatienten in Form einer global fast signifikant erniedrigten BRS. Es ist davon auszugehen, dass hohe Werte auf den Borderline-spezifischen Messinstrumenten (BPS und BPI) die bei der PTBS bekannte vagale Dysfunktion noch verstärkt. Im Unterschied zu Kuo und Linehan (2009) fanden Austin et al. (2007) keinen Unterschied des Vagotonus (RSA) zwischen BPS- Patienten und Gesunden in Ruhe. Austin et al. (2007) zeigten Borderline-Patienten des Weiteren Videoclips mit unterschiedlichem emotionalen Inhalt und bestimmten dabei die RSA als parasympathischen Marker. Die gesunde Vergleichsgruppe entwickelte im Verlauf des Experiments einen steigenden vagalen Einfluss auf das Herz, während die vagale Kontrolle in der BPS-Gruppe abnahm. Dieser parasympathische Rückzug in der BPS-Gruppe ist dem physiologischen Status von Mobilisierung (fight and flight) zuzuordnen. Im 
Gegensatz dazu wäre der steigende Vagotonus der Kontrollgruppe als ein spontanes soziales Interaktionsverhalten $\mathrm{zu}$ verstehen. Austin et al. (2007) schlussfolgerten, dass die BPS mit Schwierigkeiten der vagalen Regulation in sozialen Settings assoziiert ist. Diese Ergebnisse können ansatzweise auch auf das hier untersuchte Babyschreien übertragen werden. Während die PTBSKontrollgruppe ohne ausgeprägte Borderline-Symptomatik eine vagale Modulation während des Babyschreiens aufweist (signifikanter Anstieg der BRS Up-Events im Vergleich zur Ruhephase 1), ist diese in der Subgruppe ,,hoch Borderline“ deutlich eingeschränkt (kein BRS-Anstieg).

Tritt zu einer (k)PTBS eine ausgeprägte Borderline-Symptomatik hinzu, muss neben einer erhöhten Symptombelastung ermittelt durch Messinstrumente zu Borderline-spezifischen, depressiven und dissoziativen Symptomen auch mit einer stärker veränderten Pathophysiologie unter Stressanforderung gerechnet werden. Insbesondere das Babyschreien löste bei den Patientinnen der Subgruppe „hoch Borderline“ ein anderes Reaktionsmuster als bei den restlichen Patientinnen aus, welches ansatzweise mit einem Arousal während des Rechentests bzw. Borderline-spezifischer Stimuli vergleichbar ist mit graphischem Anstieg von HR, sBP und TPRI sowie Abfall der BRS und SDNN. Die Kontrollgruppe zeigte auf Babyschreien eher ein Reaktionsmuster im Sinne einer vagalen Aktivierung (signifikante Zunahme der BRS). Es zeigten sich signifikant unterschiedliche Änderungen zwischen den Gruppen im Paarvergleich Rechnen vs. Babyschreien für den sBP, den TPRI und annähernd für die BRS. Während in der Kontrollgruppe eine parasympathisch modulierte Aktivierung des Bindungssystems durch das Babyschreien vermutet werden kann, scheint der Subgruppe „hoch Borderline“ dieses Stressbewältigungssystem weniger zur Verfügung zu stehen. Eine ausgeprägte Borderline-Symptomatik ist vermutlich mit einem niedrigeren Vagotonus (fast signifikant herabgesetzte BRS) und einer insgesamt eingeschränkteren HRV (niedrigere SDNN und HRVParameter aus dem Frequenzbereich) assoziiert. Die für die PTBS oftmals postulierte vagale Dysfunktion konnte in der vorliegenden Studie bei einer spezifischen Subgruppe mit zusätzlich stark ausgeprägten Borderline-Merkmalen im Vergleich zu PTBS-Patienten ohne Borderline-Symptome verstärkt ausgeprägt nachgewiesen werden. Vor dem Hintergrund dieser Ergebnisse erscheint es sinnvoll, bei Patientinnen mit Traumafolgestörungen eine explizite BPS-Diagnostik zu betreiben, um in einem multimodalen Therapiekonzept auch Borderline-spezifische Symptome wie eine gestörte Affekt- und Impulsregulation anzugehen und im Folgeschluss ein physiologisches Hyperarousal in Alltagsstresssituation vermeiden zu können. Da beide Distress-Systeme, das Furcht- und das Bindungssystem, bei dieser Patientinnengruppe besonders beeinträchtigt zu sein scheinen, sollte die Therapie sowohl Anti-Furcht- als auch Anti-Panik-Anteile enthalten (Müller und Sachsse 2009). 


\subsection{Stärken, Schwächen und Ausblick}

Die vorliegende Studie hat viele Stärken, weist aber auch einige Limitationen auf, die im Folgenden erläutert werden sollen. Gleichzeitig sollen offen gebliebene Fragen angesprochen und Anregungen für künftige Studien gegeben werden.

Mit 54 Patientinnen war die Stichprobengröße im Vergleich zu vorangegangen psychophysiologischen Studien zur PTBS relativ groß und es wurde daher eine zufriedenstellende statistische Power für das Gesamtkollektiv erreicht. Zwar wurden in dieser Studie nur Frauen mit (k)PTBS bzw. BPS untersucht und damit ist die Generalisierbarkeit auf beide Geschlechter eingeschränkt, es sollte jedoch berücksichtigt werden, dass sich der Großteil der vorangegangenen Studien bisher schwerpunktmäßig auf männliche Kriegsveteranen fokussierte. Zudem haben Frauen generell ein höheres Risiko, nach einem traumatischen Ereignis eine PTBS zu entwickeln (Kessler et al. 1995).

Besonders positiv hervorzuheben ist die ausführliche Diagnostik der Patientinnen auf der spezialisierten Trauma-Station des AFG nicht nur anhand von zahlreichen standardisierten und gut validierten Fragebögen sondern auch anhand ausgewählter strukturierter Interviews. Die Diagnose (k)PTBS ist im untersuchten Kollektiv somit deutlich fundierter als in einigen Studien, die ausschließlich Selbstbeurteilungs-Fragebögen verwendeten. Es erfolgte jedoch keine differentialdiagnostische Unterscheidung von chronifizierter PTBS, komplexer PTBS und BPS. Dies bleibt künftigen Studien vorbehalten. Neben den Traumafolgestörungen wurden hier weitere Nebendiagnosen erfasst. Aufgrund der großen Anzahl von Komorbiditäten im untersuchten PTBSPatientinnenkollektiv - vor allem in Bezug auf Depression und Angst - ist ein Einfluss anderer psychischer Störungen auf die erhobenen kardiovaskulären Daten nicht sicher auszuschließen. Es wird immer wieder diskutiert, ob die physiologische Komponente der Angst allen Angststörungen gemeinsam ist und die erhobenen Ergebnisse daher nicht als PTBS-spezifisch einzuordnen sind. Auch bei Patienten mit depressiven Störungen wurde beispielsweise immer wieder eine erniedrigte HRV gefunden (Ehrenthal et al. 2010a, Kemp et al. 2010). Der Einfluss von einigen Komorbiditäten auf die psychophysiologische Reaktivität und das PTBS-typische Hyperarousal konnte jedoch auch schon öfters widerlegt werden: Metzger et al. (1999) fanden nach Adjustierung von selbst beurteilter Angst und depressiver Symptome keinen Einfluss auf die Ergebnisse und sahen sie daher als PTBSspezifisch an. Auch Patienten mit Panikstörungen zeigten nicht das gleiche pathophysiologische Reaktionsmuster wie PTBS-Patienten im Rahmen von Recall-Stresstests (Cohen et al. 2000, Blechert et al. 2007). Hughes et al. (2007) identifizierten eine niedrige Ruhe-BRS als PTBS-spezifisch, da das Vorhandensein bzw. die Abwesenheit einer komorbiden Depression keine Änderung der Ergebnisse erbrachte. In Folgestudien sollte nichts desto trotz der Einfluss von komorbiden Störungen auf die psychophysiologische Reagibilität genauer untersucht werden.

Ein weiterer Vorteil der Studie ist, dass das Patientinnenkollektiv eine große Heterogenität in Bezug auf die Traumaentität aufweist, sodass die Ergebnisse breiter übertragbar sind und nicht nur für bestimmte, bisher oft untersuchte Gruppen wie Kriegsveteranen (z.B. Keane et al. 1998, Peckerman et 
al. 2003, Tan et al. 2011) oder Motorradunfallopfer (z.B. Blanchard et al. 1996, Shaikh al arab et al. 2012) gelten, sondern auch insbesondere Typ-II-Traumatisierungen einschließen.

$\mathrm{Zu}$ den größten Stärken der vorliegenden Studie zählt die differenzierte multidimensionale Betrachtung der Stressreagibilität der traumatisierten Frauen. In bisherigen Studien wurde der Begriff „psychophysiologisches Arousal“ sehr allgemein verwendet und nur einzelne Herzkreislauf-Parameter untersucht. Hier erfolgte nun mittels TFM-Messung eine Analyse in fünf verschiedenen Dimensionen: vorwiegend beta-adrenerg (HR und $\mathrm{CI}$ ), vorwiegend alpha-adrenerg (sBP, dBP, mBP und TPRI), HRV-Analyse sowohl im Zeit- als auch im Frequenzbereich sowie BRS. Der Task Force Monitor ist ein gut validiertes Messinstrument (Fortin et al. 2001) und erwies sich hier auch bei PTBSPatientinnen unter psychischer Stressbelastung als praktikabel. Alle hämodynamischen Parameter wurden in einer Beat-to-Beat-Basis erfasst und in Echtzeit wiedergegeben. Besonders hervorzuheben ist die nicht-invasive kontinuierliche Blutdruckmessung des TFM, die sich als äquivalent zu anerkannten Blutdruckmesssystemen herausstellte (Fortin et al 2001). Neben der Erfassung von Blutdruckänderungen peripher erfolgt automatisch eine Korrektur mit dem oszillometrischen Blutdruck am Oberarm. In vorangegangenen Studien erfolgte die Blutdruckmessung meistens diskontinuierlich mit alter Technik und ist daher weniger präzise. Trotz guter Praktikabilität des TFM gab es doch einige methodische Probleme wie kurzzeitige Ausfälle von Messsignalen. Erwartungsgemäß waren die Impedanzkardiographie sowie der Blutdruck (Vascular-UnloadingTechnik) anfälliger als das EKG. Es erfolgte deshalb eine ausführliche Einzelfallanalyse und Plausibilitätskontrolle, um eine valide und repräsentative Bildung von Phasenmittelwerten gewährleisten zu können. Es erfolgte ein direkter Vergleich verschiedener Marker für das periphere Nervensystem (SDNN, LF- und HF-HRV sowie BRS), welcher in Vorgängerstudien oftmals als fehlend bemängelt worden war. Insbesondere der Vergleich von HF-HRV und BRS ist hier zu nennen. Da es bereits zahlreiche HRV-Studien mit Parametern aus dem Frequenzbereich gibt, wurde zusätzlich Wert auf eine Auswertung der SDNN aus dem Zeitbereich gelegt. Die SDNN ist eine direkte, einfache, praktische und zugleich auch präzisere Methode, um autonome Funktionen zu beurteilen, da eine Power-Spektralanalyse zur Beurteilung von HRV-Parametern aus dem Frequenzbereich zwangsläufig durch Logarithmieren der Werte mit einer Abschwächung der statistischen Power einhergeht (Kuss et al. 2008). Darüber hinaus sollte bedacht werden, dass die LF-HRV keine rein sympathisch modulierte HRV-Komponente ist. Es gibt Kritiker, die keine Vergesellschaftung von Adrenalin-Level und LF-HRV postulieren (Sloan et al. 1996). Für eine präzise Unterscheidung von sympathischen und parasympathischen Einflüssen auf die Herzfrequenz könnten künftige Studien zusätzlich pharmakologische Blockaden einsetzen. Die Frage, ob die beobachtete gestörte physiologische Reagibilität der (k)PTBS-Patientinnen konstitutionell oder erworben ist, kann mit dem vorliegendem Studiendesign nicht klärend beantwortet werden. Entweder führt eine PTBS wie hier angenommen zu einer eingeschränkten vagalen Modulationsfähigkeit oder aber eine präexistente reduzierte HF-HRV bzw. BRS stellt einen Risikofaktor für die Entwicklung einer PTBS dar. Die hier 
vorgestellte multidimensionale Testung von Alltagsstressoren (Rechnen und Babyschreien) sollte in folgenden Untersuchungen um andere Alltags- sowie traumaassoziierte Stressoren erweitert werden. Inzwischen sind viele Faktoren wie beispielsweise Rauchen, Koffein oder Medikamente bekannt, die in der Lage sind, die sympathovagale Balance zu beeinflussen (Cohen et al. 1997, Löllgen 1999). Es war nicht möglich, im Rahmen des Studiendesigns alle Einflussfaktoren, die die HRV ggf. verändern können, zu kontrollieren. Die bewusst eng gewählten Ausschlusskriterien haben demnach sowohl Vorteile als auch Nachteile. Da das Alter gemäß der Framingham Studie (Tsuji et al. 1996) als maßgebliche Determinante der HRV gilt, wurde hier im Vergleich zu anderen Studien eine obere Altersgrenze von 59 Jahren festgelegt. Die Medikamenteneinnahme der Patientinnen wurde dokumentiert, sie führte jedoch nicht zum Studienausschluss. Der Einfluss anticholinerg und stark herzwirksamer Medikamente war nicht Gegenstand der vorliegenden Arbeit, wurde jedoch in einer parallel durchgeführten Auswertung am gleichen Patientinnenkollektiv von G. Bornschein untersucht. Es ist möglich, dass eine Medikamenteneinnahme die physiologische Reaktivität einzelner Teilnehmerinnen reduzierte bzw. veränderte. Vorgenommene Medikamentenadjustierungen vorangegangener Studien erbrachten jedoch keinen wesentlichen Einfluss der Medikamente auf die Ergebnisse von HR (z.B. Metzger et al. 1999), BRS (Hughes et al. 2007) oder RSA (Sahar et al. 2001). In der vorliegenden Studie erfolgte auch keine Anweisung an die Patientinnen, ihren Nikotinbzw. Koffeingenuss vor Durchführung der Untersuchung zu unterbinden. In Vorgängerstudien zeigte sich jedoch kein Einfluss von Nikotin (Ehrenthal et al. 2010a, Hughes et al. 2007) oder Koffein (Hopper et al. 2006) auf autonome Parameter. Weiterhin erfolgte keine Kontrolle des Einflusses von Respiration und Atemveränderungen während des Arousal oder durch das Nutzen von Atemübungen als Copingmechanismen, welche die HRV und die BRS beeinflusst haben könnten (Bernardi et al. 2002). Ein großer Vorteil der wenigen Ausschlusskriterien ist die so gewährleistete bessere Generalisierbarkeit, denn das Patientinnenkollektiv ist somit deutlich repräsentativer für typische PTBS-Patienten, die bekanntermaßen sehr häufig rauchen und Psychopharmaka oder andere Medikamente einnehmen. Somit sind die Ergebnisse besser auf reale, alltägliche Gegebenheiten übertragbar. Während viele Studien die Reagibilität auf spezifische traumaassoziierte Stimuli untersuchten, wurden hier bewusst zwei Alltagsstressoren aus dem kognitiven (Kopfrechnen) und emotionalen (Babyschreien) Bereich gewählt. Das Babyschreien wurde unseres Wissens bisher in keiner PTBS-Studie angewandt und erwies sich als praktikabler Stresstest, der Hinweise auf unterschiedliche Reaktionsmuster bieten kann. Durch den Versuchsaufbau mit mehreren Ruhephasen zwischen den Stresstests war es möglich, auch die Rückbildungsfähigkeit nach Stressanforderung (Recovery) der Patientinnen zu beurteilen. Positiv zu erwähnen ist die eingesetzte Analogskala zum Stressempfinden der Patientinnen während der verschiedenen Abschnitte der Stressbelastungsuntersuchung, die es ermöglichte, neben der physiologischen auch die subjektive Aktivierung der Patientinnen zu erfassen. Trotz der teilweise sehr großen subjektiven Stressbelastung 
für diese emotional labile Patientinnengruppe, war die Mitarbeit der Patientinnen sehr gut und nur wenige mussten die Stresstests vorzeitig abbrechen.

Eine weitere Besonderheit dieser Studie ist die Untersuchung einer Patientinnen-Untergruppe mit stark ausgeprägter Borderline-Symptomatik im Vergleich zum Rest des Ausgangskollektivs. Zur Bildung der Subgruppe „hoch Borderline“ wurde sich an allgemein üblichen Messinstrumenten für die Borderline-Persönlichkeitsstörung orientiert, dem Borderline-Persönlichkeits-Inventar (BPI, Leichsenring, 1997) und der Borderline-Symptom-Liste (BSL, Bohus et al. 2001). Als Subgruppendefinierende Kriterien wurden ein Cut-20-Wert $\geq 10$ des BPI sowie Werte auf den BSL-Subskalen Affektregulation >25 und Autoaggression >17,5 festgelegt (siehe Methodenteil, Seite 34). Soweit bekannt gibt es nur zwei weitere Studien, die die psychophysiologische Reaktivität von PTBS und BPS gegenüberstellend untersuchten (Schmahl et al. 2004, Limberg et al. 2011). Generell wurde die Betrachtung von PTBS-Subtypen bislang weitestgehend vernachlässigt. In Bezug auf die SubgruppenBildung ergibt sich hier jedoch eine relativ kleine Stichprobengröße, sodass die statistische Power nicht ausreichend gewesen wäre für eine zusätzliche Analyse der Effekte anderer psychometrischer Merkmale auf die Stressphysiologie. Zukünftige Kollektivgrößen sollten noch größer angesetzt werden, sodass verschiedene abgrenzbare Subgruppen mit unterschiedlich stark ausgeprägten psychometrischen Befunden gebildet werden können. Interessant wären neben einer ausgeprägten Borderline-Symptomatik auch hoch dissoziative, depressive sowie intrusive Eigenschaften.

Zusammenfassend lässt sich festhalten, dass PTBS-Patientinnen eine pathophysiologische Stressreagibilität, multiple Komorbiditäten, partnerschaftliche und familiäre Dysfunktionen, berufliche Beeinträchtigung sowie ein erhöhtes Risiko für kardiovaskuläre Erkrankungen aufweisen und daher weitere Bemühungen, dieses erhebliche öffentliche Gesundheitsproblem zu verstehen, zu messen und zu therapieren unabdingbar sind. Die hier erhobenen Befunde erfordern eine Wiederholung und Erweiterung in Bezug auf Stimuli, Einfluss von Komorbiditäten und Confounder sowie Subgruppen in nachfolgenden Untersuchungen.

Eventuell ergibt sich aus der hier vorgestellten Stressbelastungsuntersuchung mittels Task Force Monitor zur Erfassung hämodynamischer und autonomer Parameter in Ruhe sowie unter Einsatz verschiedener Alltagsstressoren eine diagnostische Möglichkeit, um Patientinnen mit Traumafolgestörungen besser in spezifische Subgruppen einteilen zu können. Eine aktuelle MultiCenter-Studie hat ergeben, dass eine Differenzierung der diagnostischen Cluster kPTBS und BPS nicht eindeutig möglich ist, beide Entitäten also vermutlich die gleiche Patientengruppe zu erfassen versuchen (Sack et al. 2012). Möglicherweise könnten durch eine psychophysiologische Datenerhebung aber hyper- bzw. hyporeagible Patienten identifiziert und entsprechende individuelle Behandlungskonzepte entworfen werden. Eine psychophysiologische Untersuchung mittels TFM ließe sich als ergänzende Diagnostik zu Selbstbeurteilungs-Messinstrumenten und strukturierten Interviews etablieren, um eine initiale umfangreichere Beurteilung der PTBS und ggf. eine Vorhersage des PTBS- Schweregrads zu erreichen. Auch eine Erfolgsüberprüfung der spezifischen Traumatherapie 
anhand von TFM-Werten wäre denkbar. Ehrenthal et al. (2010b) stellten beispielsweise den Nutzen kardiovaskulärer Messungen zur Vorhersage des Behandlungserfolges von depressiven Patienten heraus. Es gilt zu überprüfen, ob die psychophysiologische Stressreaktivität auch als Prädiktor für die Entwicklung einer Borderline-Symptomatik bei (k)PTBS dienen kann. In der geplanten Follow-UpStudie wird das PTBS-Patientinnenkollektiv nach Erhalt einer multimodalen Traumatherapie erneut auf ihre Psychophysiologie hin untersucht. Dies kann ggf. Auskunft über Therapieerfolg und mögliche Einflussfaktoren für eine Chronifizierung der PTBS-Symptomatik geben. 


\section{Zusammenfassung}

Komplexen Traumafolgestörungen liegt eine gestörte Stressverarbeitung mit sympathovagaler Dysregulation zugrunde, die in banalen Belastungssituationen $\mathrm{zu}$ vegetativen Übererregungssymptomen (Hyperarousal) oder dissoziativen Reaktionen führt. Die Ergebnisse vorangegangener Studien zur Psychophysiologie bei PTBS sind jedoch uneinheitlich, umfassen oftmals nur einzelne Herz-Kreislauf-Parameter und haben lange Zeit den starken Einfluss des Parasympathikus vernachlässigt. Die vorliegende Studie nutzte ein multidimensionales Untersuchungsmodell zur besseren Erfassung der Stressreagibilität bei Frauen mit (k)PTBS bzw. BPS. Es wurden insgesamt 54 Patientinnen in die Studie eingeschlossen, bei denen anhand strukturierter Interviews und Fragebogendiagnostik eine (k)PTBS und ggfs. andere komorbide Traumafolgestörungen diagnostiziert wurden. Es erfolgte eine nicht-invasive kardiovegetative Funktionsmessung in Ruhe, unter Einwirkung verschiedener Stressoren (Rechentest und Babyschreien), in anschließenden Erholungsphasen sowie während einer Entspannungsmusik. Für eine umfassende Beurteilung der psychophysiologischen Aktivierung der PTBS-Patientinnen wurden sowohl alpha- und beta-adrenerge Reaktionsparameter als auch HRV-Parameter aus dem Zeit- sowie Frequenzbereich und die Barorezeptorsensitivität ausgewertet. Darüber hinaus wurde eine anhand psychometrischer Merkmale gebildete Subgruppe „hoch Borderline“ in Gegenüberstellung zum Rest des Ausgangskollektivs ohne ausgeprägte Borderline-Symptomatik untersucht.

Durch Simulation alltäglicher Stressbelastungen war bei den Patientinnen eine subjektive, emotionale sowie psychophysiologische Aktivierung auf verschiedenen Ebenen induzierbar. Während des kognitiven Stressors „Rechnen“ zeigte sich zwar eine sympathische Aktivierung mit signifikanter Zunahme von HR, CI, sBP und LF-HRV, der erwartete parasympathische Rückzug unter Stress war jedoch eingeschränkt (keine signifikante Abnahme von SDNN, HF-HRV und BRS), sodass eine dysfunktionale vagale Modulation vermutet werden kann. Während der emotionalen Stresseinwirkung „Babyschreien“ wurde hingegen ein Absinken der vorwiegend beta-adrenergen Parameter sowie eine vagale Dominanz (Zunahme der BRS, Abnahme der LF-HRV) beobachtet, sodass sich für diese Parameter signifikante Paarvergleiche zwischen den beiden Stressoren ergaben. Zudem wurden während des Babyschreiens vermehrt vegetative bzw. emotionale Reaktionen und im Anschluss eine verzögerte Rückbildungsfähigkeit der Stressparameter beobachtet. Eine zusätzlich zur (k)PTBS vorliegende ausgeprägte Borderline-Symptomatik erhöhte die Symptombelastung (Borderlinespezifisch, depressiv und dissoziativ) der Patientinnen. Die für die PTBS bekannte vagale Dysfunktion scheint bei den Patientinnen der Subgruppe „hoch Borderline“ verstärkt (herabgesetzte BRS). Des Weiteren wiesen die hoch Borderline-Patientinnen im Vergleich zu den restlichen Patientinnen einen signifikant erhöhten Blutdruck auf. Beim Paarvergleich der beiden Stressoren (Rechentest vs. Babyschreien) zeigten sich im Gruppenvergleich signifikant unterschiedliche Phasenmittelwert- 
Differenzen für den SBP und den TPRI sowie fast signifikant unterschiedliche Änderungen der BRS, was ein unterschiedliches Reaktionsmuster zwischen den Gruppen während der Stresseinwirkung „Babyschreien“ unterstreicht.

Zusammenfassend zeigt sich, dass bei der (k)PTBS nicht allein, wie lange angenommen, eine gesteigerte sympathische Aktivierung für ein Hyperarousal verantwortlich ist, sondern defizitäre parasympathische Einflüsse eine entscheidende Rolle spielen. Beide Distress-Systeme, das Furchtund das Bindungssystem, scheinen insbesondere bei komorbider Borderline-Symptomatik beeinträchtigt zu sein. Die hier erhobenen Befunde erfordern eine Wiederholung und Erweiterung in Bezug auf Stimuli, Einfluss von Komorbiditäten und Confounder sowie spezifische Subgruppen in nachfolgenden Studien. 


\section{$7 \quad$ Literaturverzeichnis}

Allen K, Golden LH, Izzo JL Jr, Ching MI, Forrest A, Niles CR, Niswander PR, Barlow JC (2001): Normalization of hypertensive responses during ambulatory surgical stress by perioperative music. Psychosom Med $\underline{63}$, 487-92

APA - American Psychiatric Association: Diagnostic and Statistical Manual of Mental Disorders DSM-III. American Psychiatric Association, Washington DC 1980

APA - American Psychiatric Association: Diagnostic and Statistical Manual of Mental Disorders DSM-IV. Deutsche Übersetzung hrsg. v. Saß H, Wittchen HU, Zaudig M, Houben I: Diagnostisches und Statistisches Manual Psychischer Störungen DSM-IV. Hogrefe, Göttingen 2003

Austin MA, Riniolo TC, Porges SW (2007): Borderline personality disorder and emotion regulation: insights from the Polyvagal Theory. Brain Cogn $\underline{65}$, 69-76

Baule G: Wirkung einer Klang-Vibrationsliege auf psychologische und physiologische Parameter. Psychologische Diplomarbeit, Göttingen 2003

Beck AT, Ward CH, Mendelson, M (1961): An inventory for measuring depression. Arch Gen Psychiatry $4,561-571$

Behrens GA (2008): Using music therapy to understand emotional needs of Palestinian children traumatized by war. Paper presented at a meeting of the American Music Therapy Association, St. Louis 2008

Bernardi L, Porta C, Spicuzza L, Bellwon J, Spadacini G, Frey AW, Yeung LY, Sanderson JE, Pedretti R, Tramarin R (2002): Slow breathing increases arterial baroreflex sensitivity in patients with chronic heart failure. Circulation 105, 143-5

Bernardi L, Porta C, Sleight P (2006): Cardiovascular, cerebrovascular, and respiratory changes induced by different types of music in musicians and non-musicians: the importance of silence. Heart 92, 445-52

Bernstein EM, Putnam FW (1986): Development, reliability, and validity of a dissociation scale. J Nerv Ment Dis $174,727-735$

Blanchard EB, Hickling EJ, Buckley TC, Taylor AE, Vollmer A, Loos WR (1996): Psychophysiology of posttraumatic stress disorder related to motor vehicle accidents: replication and extension. $\mathrm{J}$ Consult Clin Psychol 64, 742-751

Blechert J, Michael T, Grossman P, Lajtman M, Wilhelm FH (2007): Autonomic and respiratory characteristics of posttraumatic stress disorder and panic disorder. Psychosom Med 69, 935-43

Bockers E, U, Jacobs S, Strack M (2008): Dissoziativität - zur Sensitivität und Spezifität des FDS. Persönlichkeitsstörungen - Theorie und Therapie (PTT) 12, 186-188

Bohus M, Limberger M, Sender I, Gratwohl T, Stieglitz R (2001): Entwicklung der BorderlineSymptom-Liste. Psychother Psychosom Med Psychol 1ㅣ, 201-211

Bohus M, Limberger M, Frank U, Chapman A, Kühler T, Stieglitz RD (2007): Psychometric properties of the Borderline Symptom List (BSL). Psychopathology 40, 126-132

Brandao ML, Zanoveli JM, Ruiz-Martinez RC, Oliveira LC, Landeira-Fernandez J (2008): Different patterns of freezing behavior organized in the periaqueductal gray of rats: association with different types of anxiety. Behav Brain Res $\underline{188}, 1-13$

Bremner JD, Steinberg M, Southwick SM, Johnson DR, Charney DS (1993): Use of the Structured Clinical Interview for DSM-IV Dissociative Disorders for systematic assessment of dissociative symptoms in posttraumatic stress disorder. Am J Psychiatry 150, 1011-1014 
Bremner JD, Randall PR, Scott TM, Bronen RA, Seibyl JP, Southwick SM, Delaney RC, McCarthy G, Charney DS, Innis RB (1995): MRI-based measurement of hippocampal volume in patients with combat-related posttraumatic stress disorder. Am J Psychiatry 152, 973-981

Bremner JD, Randall PR, Vermetten E, Staib L, Bronen RA, Mazure C, Capelli S, McCarthy G, Innis RB, Charney DS (1997): Magnetic resonance imaging-based measurement of hippocampal volume in posttraumatic stress disorder related to childhood physical and sexual abuse-a preliminary report. Biol Psychiatry 41, 23-32

Brewster AL, Nelson JP, McCanne TR, Lucas DR, Milner JS (1998): Gender differences in physiological reactivity to infant cries and smiles in military families. Child Abuse Negl $\underline{22}$, $775-88$

Boroske-Leiner K, Hofmann A, Sack M (2008): Ergebnisse zur internen und externen Validität des Interviews zur komplexen Posttraumatischen Belastungsstörung (I-kPTBS). Psychother Psychsom Med Psychol 58, 192-199

Cannon WB (1914): The emergency function of the adrenal medulla in pain and the major emotions. Am J Physiol 33, 356-372

Carr C, d'Ardenne P, Sloboda A, Scott C, Wang D, Priebe S (2012):Group music therapy for patients with persistent post-traumatic stress disorder - an exploratory randomized controlled trial with mixed methods evaluation. Psychol Psychother $\underline{85}$, 179-202

Casanova GM, Domanic J, McCanne TR, Milner JS(1994): Physiological responses to child stimuli in mothers with and without a childhood history of physical abuse. Child Abuse Negl 18, 9951004

Cohen H, Kotler M, Matar MA, Kaplan Z, Miodownik H, CassutoY (1997): Power spectral analysis of heart rate variability in posttraumatic stress disorder patients. BiolPsychiatry 41, 627-629

Cohen H, Kotler M, Matar MA, Kaplan Z, Loewenthal U, Miodownik H, Cassuto Y (1998): Analysis of heart rate variability in posttraumatic stress disorder patients in response to a trauma-related reminder. Biol Psychiatry $\underline{44}, 1054-1059$

Cohen H, Benjamin J, Geva AB, Matar MA, Kaplan Z, Kotler M (2000): Autonomic dysregulation in panic disorder and in post-traumatic stress disorder: application of power spectrum analysis of heart rate variability at rest and in response to recollection of trauma or panic attacks. Psychiatry Res $\underline{96}, 1-13$

Cole CR, Blackstone EH, Pashkow FJ, Snader CE, Lauer MS (1999): Heart-rate recovery immediately after exercise as a predictor of mortality. N Engl J Med $\underline{341}, 1351-7$

Crowe HP, Zeskind PS (1992): Psychophysiological and perceptual responses to infant cries varying in pitch: comparison of adults with low and high scores on the Child Abuse Potential Inventory. Child Abuse Negl 16, 19-29

Da Costa JM (1871): On irritable heart: a clinical study of a form of functional cardiac disorder and its consequences. Am J Med Sci $\underline{61}$, 17-52

Dammann G, Overkamp B: Diagnose, Differentialdiagnose und Komorbidität dissoziativer Störungen des Bewusstseins. In: Psychotherapie der dissoziativen Störungen, hrsg. v. Reddemann L, Hofmann A, Gast U. Thieme, Stuttgart 2004, 3-25

Del Vecchio T, Walter A, O'Leary SG (2009): Affective and physiological factors predicting maternal response to infant crying. Infant Behav Dev $\underline{32}, 117-22$

Derogatis L, Lipman RS, Covi L (1973): SCL-90: A outpatient psychiatric rating scale - Preliminary report. Psychopharmacol Bull $\underline{9}, 13-28$

Donovan WL, Leavitt LA, Walsh RO (1998): Conflict and depression predict maternal sensitivity to infant cries. Infant Behav Dev 21, 505-17

Driessen M, Beblo T, Reddemann L, Rau H, Lange W, Silva A, Berea RC, Wulff H, Ratzka S (2002): Ist die Borderline-Persönlichkeitsstörung eine komplexe posttraumatische Störung? Zum Stand der Forschung. Nervenarzt $\underline{73}$, 820-829 
Ehlers A, Suendermann O, Boellinghaus I, Vossbeck-Elsebusch A, Gamer M, Briddon E, Martin MW, Glucksman E (2010): Heart rate responses to standardized trauma-related pictures in acute posttraumatic stress disorder. Int J Psychophysiol 78, 27-34

Ehrenthal JC, Herrmann-Lingen C, Fey M, Schauenburg H (2010a): Altered cardiovascular adaptability in depressed patients without heart disease. World J Biol Psychiatry 11, 586-93

Ehrenthal JC, Fey M, Herrmann-Lingen C, Schauenburg H (2010b): Psychophysiologie als Prädiktor für Behandlungserfolg: Eine Pilotstudie. Psychother Psychosom Med Psychol 60, 474-8

Elices M, Soler J, Fernández C, Martín-Blanco A, Jesús Portella M, Pérez V, Alvarez E, Carlos Pascual J (2012): Physiological and self-assessed emotional responses to emotion-eliciting films in borderline personality disorder. Psychiatry Res 므, 437-43

Feldman R, Weller A, Zagoory-Sharon O, Levine A (2007): Evidence for a neuroendocrinological foundation of human affiliation: plasma oxytocin levels across pregnancy and the postpartum period predict mother-infant bonding. Psychol Sci 18, 965-970

Fischer G, Riedesser P: Lehrbuch der Psychotraumatologie. Ernst Reinhardt Verlag, München 1998, 79

Fortin J, Haitchi G, Bojic A, Habenbacher W, Grüllenberger R, Heller A, Pacher R, Wach P, Skrabal F (2001): Validation and Verification of the Task Force Monitor. Results of Clinical Studies for FDA 510 (k) no: K014063, 1-7

Fortin J, Marte W, Grüllenberger R, Hacker A, Habenbacher W, Heller A, Wagner Ch, Wach P, Skrabal F (2006a): Continuous non-invasive blood pressure monitoring using concentrically interlocking control loops. Comput Biol Med $\underline{36}, 941-57$

Fortin J, Habenbacher W, Heller A, Hacker A, Grüllenberger R, Innerhofer J, Passath H, Wagner C, Haitchi G, Flotzinger D (2006b): Non-invasive beat-to-beat cardiac output monitoring by an improved method of transthoracic bioimpedance measurement. Comput Biol Med $\underline{36}$, 11851203

Franke GH: Die Symptom-Checkliste von Derogatis. Deutsche Version - Manual. Beltz, Göttingen 1995

Franke GH (2001): Möglichkeiten und Grenzen im Einsatz der Symptom-Checkliste SCL-90-R. VPP $\underline{33}, 475-485$

Freyberger HJ, Spitzer C, Stieglitz RD: Der Fragebogen zu dissoziativen Symptomen (FDS). Hans Huber Verlag, Bern 1999

Frodi AM, Lamb ME (1980): Child abusers' responses to infant smiles and cries. Child Dev $\underline{51}$, 23841

Frör K: Dissoziative Identitätsstörung - eine polysymptomatische Störung? Eine Validierungsstudie mit dem SIDDS. Diplomarbeit Psychologie, Marburg 2000

Geuze E, Vermetten E, Bremner JD (2005): MR-based in vivo hippocampal volumetrics: Findings in neuropsychiatric disorders. Mol Psychiatry 10, 160-184

Gratze G, Fortin J, Holler A, Grasenick K, Pfurtscheller G, Wach P, Schönegger J, Kotanko P, Skrabal F (1998): A software package for non-invasive, real-time beat-to-beat monitoring of stroke volume, blood pressure, total peripheral resistance and for assessment of autonomic function. Comput Biol Med $\underline{28}, 121-142$

Gunderson JG, Kolb JE, Austin V (1981): The Diagnostic Interview for Borderline Patients. Am J Psychiatry $\underline{138}, 896-903$

Gutner CA, Pineles SL, Griffin MG, Bauer MR, Weierich MR, Resick PA (2010): Physiological predictors of posttraumatic stress disorder. J Trauma Stress $\underline{23}, 775-84$

Heffernan K, Cloitre M (2000): A comparison of posttraumatic stress disorder with and without borderline personality disorder among women with a history of childhood sexual abuse: Etiological and clinical characteristics. J Nerv Ment Dis $\underline{188}$, 589-595

Herman JL: Trauma and Recovery. Basic Books, New York 1992 
Herman JL: Die Narben der Gewalt. Traumatische Erfahrungen verstehen und überwinden. Junfermann, Paderborn 2003

Herpertz SC, Kunert HJ, Schwenger UB, Sass H (1999): Affective responsiveness in borderline personality disorder: a psychophysiological approach. Am J Psychiatry 156, 1550-6

Herrmann-Lingen C (2000): Biopsychosoziale Faktoren in Genese und Manifestation der Koronaren Herzkrankheit. Z Psychosom Med Psychother 46, 315-330

Herrmann-Lingen C, Lomb J und Schunk T (2010): Multidimensionality of mental stress-induced autonomic activation in a psychosomatic patient population. Psychosom Med $\underline{72}$, A-128

Hilz MJ, Dütsch M (2006): Quantitative studies of autonomic function. Muscle Nerve 33, 6-20

Hopper JW, Spinazzola J, Simpson WB, van der Kolk BA (2006): Preliminary evidence of parasympathetic influence on basal heart rate in posttraumatic stress disorder. J Psychosom Res $\underline{60}, 83-90$

Horowitz MJ, Wilner N, Alvarez W (1979): Impact of Event Scale: A measure of subjective stress. Psychosom Med 41, 209-218

Hüther G (1996): The central adaption syndrome. Psychosocial modification and reorganization of neuronal networks. Psychiatry Res $\underline{87}, 83-95$

Hüther G, Sachsse U (2007): Angst- und stressbedingte Störungen. Auf dem Weg zu einer neurobiologisch fundierten Psychotherapie. Psychotherapeut 52, 166-179

Hughes JW, Feldman ME, Beckham JC (2006): Posttraumatic stress disorder is associated with attenuated baroreceptor sensitivity among female, but not male, smokers. Biol Psychol $\underline{71}$, 296-302

Hughes JW, Dennis MF, Beckham JC (2007): Baroreceptor sensitivity at rest and during stress in women with posttraumatic stress disorder or major depressive disorder. J Trauma Stress $\underline{20}$, 667-76

Irle E, Lange C, Sachsse U, Weniger G (2009): Further evidence that post-traumatic stress disorder but not dissociative disorders are related to amygdala and hippocampal size reduction in traumaexposed individuals. Acta Psychiatr Scand 119, 330-331

Iwanaga M, Tsukamoto M (1997): Effects of excitative and sedative music on subjective and physiological relaxation. Percept Mot Skills $\underline{85}$, 287-96

Iwanaga M, Moroki Y (1999): Subjective and Physiological Responses to Music Stimuli Controlled Over Activity and Preference. J Music Ther 36, 26-38

Iwanaga M, Kobayashi A, Kawasaki C (2005): Heart rate variability with repetitive exposure to music. Biol Psychol 70, 61-6

Karl A, Schaefer M, Malta LS, Därfel D, Rohleder N, Werner A (2006a): A meta-analysis of structural brain abnormalities in PTSD. Neurosci Biobehav Rev 30, 1004-1031

Karl A, Malta LS, Maercker A (2006b): Meta-analytic review of event-related potential studies in posttraumatic stress disorder. Biol Psychol 71,123-147

Keane TM, Kolb LC, Kaloupek DG, Orr SP, Blanchard EB, Thomas RG et al. (1998): Utility of psychophysiological measurement in the diagnosis of posttraumatic stress disorder: results from a Department of Veterans Affairs Cooperative Study. J Consult Clin Psychol 66, 914923

Keary TA, Hughes JW, Palmieri PA (2009): Women with posttraumatic stress disorder have larger decreases in heart rate variability during stress tasks. Int J Psychophysiol $\underline{73}$, 257-264

Kemp AH, Quintana DS, Gray MA, Felmingham KL, Brown K, Gatt JM (2010): Impact of depression and antidepressant treatment on heart rate variability: a review and meta-analysis. Biol Psychiatry 67, 1067-74

Kernberg OF (1967): Borderline Personality Organization. Am J Psychoanal Assoc 15, 641-685

Kernberg OF: Borderline-Persönlichkeitsorganisation und Klassifikation der Persönlichkeitsstörungen. In: Handbuch der Borderline-Störungen; Kernberg OF, Dulz B, Sachsse U; Schattauer, Stuttgart 2000, 45-56 
Kessler RC, Sonnega A, Bromet E, Hughes M, Nelson CB (1995): Posttraumatic stress disorder in the National Comorbidity Survey. Arch Gen Psychiatry $\underline{52}$, 1048-60

Khalfa S, Roy M, Rainville P, Dalla Bella S, Peretz I (2008): Role of tempo entrainment in psychophysiological differentiation of happy and sad music? Int J Psychophysiol 68, 17-26

Klinke R, Silbernagl S: Lehrbuch der Physiologie; 4.Auflage. Thieme, Stuttgart 2005, 199

Knight WE, Rickard PhD NS (2001): Relaxing music prevents stress-induced increases in subjective anxiety, systolic blood pressure, and heart rate in healthy males and females. J Music Ther $\underline{38}$, 254-72

Kop WJ, Krantz DS, Nearing BD, Gottdiener JS, Quigley JF, O'Callahan M, DelNegro AA, Friehling TD, Karasik P, Suchday S, Levine J, Verrier RL (2004): Effects of acute mental stress and exercise on T-wave alternans in patients with implantable cardioverter defibrillators and controls. Circulation $\underline{109}, 1864-9$

Krumhansl CL (1997): An exploratory study of musical emotions and psychophysiology. Can J Exp Psychol 51, 336-53

Kuo JR, Linehan MM (2009): Disentangling emotion processes in borderline personality disorder: physiological and self-reported assessment of biological vulnerability, baseline intensity, and reactivity to emotionally evocative stimuli. J Abnorm Psychol 118, 531-44

Kuss O, Schumann B, Kluttig A, Greiser KH, Haerting J (2008): Time domain parameters can be estimated with less statistical error than frequency domain parameters in the analysis of heart rate variability. J Electrocardiol $\underline{41}, 287-91$

Lache B: Beitrag psychometrischer und psychophysiologischer Diagnostik zur Vorhersage arrhythmischer Ereignisse bei Patienten mit implantiertem Defibrillator. Med. Diss. Göttingen 2006

Ladwig KH, Baumert J, Marten-Mittag B, Kolb C, Zrenner B, Schmitt C (2008): Posttraumatic stress symptoms and predicted mortality in patients with implantable cardioverter-defibrillators: results from the prospective living with an implanted cardioverter-defibrillator study. Arch Gen Psychiatry 65, 1324-1330

Lanius RA, Bluhm R, Lanius U, Pain C (2006): A review of neuroimaging studies in PTSD: heterogeneity of response to symptom provocation. J Psychiat Res 40, 709-729

La Rovere MT, Mortara A, Schwartz PJ (1995): Baroreflex sensitivity. J Cardiovasc Electrophysiol $\underline{6}$, 761-774

La Rovere MT, Bigger JT Jr, Marcus FI, Mortara A, Schwartz PJ (1998): Baroreflex sensitivity and heart-rate variability in prediction of total cardiac mortality after myocardial infarction. ATRAMI (Autonomic Tone and Reflexes After Myocardial Infarction) Investigators. Lancet $\underline{351}, 478-84$

Lee KC, Chao YH, Yiin JJ, Chiang PY, Chao YF (2011): Effectiveness of different music-playing devices for reducing preoperative anxiety: a clinical control study. Int J Nurs Stud $\underline{48}, 1180-7$

Leichsenring F: Borderline-Persönlichkeits-Inventar (BPI). Manual. Hogrefe, Göttingen 1997

Leichsenring F, Chabrol H (2006): Borderline-Persoenlichkeits-Inventar (BPI) - ein Selbstbeurteilungs-Instrument zur Erfassung der Borderline-Persönlichkeitsorganisation. Persönlichkeitsstörungen - Theorie und Therapie (PTT) $\underline{10}$, 31-42

Limberg A, Barnow S, Freyberger HJ, Hamm AO (2011): Emotional vulnerability in borderline personality disorder is cue specific and modulated by traumatization. Biol Psychiatry $\underline{69}, 574-$ 82

Linehan M: Cognitive Behavioral Treatment of Borderline Personality Disorder. Guilford Press, New York 1993

Lobbestael J, Arntz A (2010): Emotional, cognitive and physiological correlates of abuse-related stress in borderline and antisocial personality disorder. Behav Res Ther $\underline{48}, 116-24$

Löllgen H (1999): Herzfrequenzvariabilität. Dtsch Ärztebl 96, A2029-32

Loranger AW: Personality Disorder Examination (PDE). Manual. Yonkers, New York 1988 
Loranger AW: IPDE: International Personality Disorder Examination: DSM-IV and ICD-10 Interviews. Psychological Assessment Resources, Odessa 1999

Loranger AW, Sartorius N, Andreoli A, Berger P, Buchheim P, Channabasavanna SM, Coid B, Dahl A, Diekstra RF, Ferguson B (1994): The International Personality Disorder Examination. The World Health Organization/Alcohol, Drug Abuse, and Mental Health Administration international pilot study of personality disorders. Arch Gen Psychiatry 51, 215-24.

Lyons-Ruth K (2008): Contributions of the mother-infant relationship to dissociative, borderline, and conduct symptoms in young adulthood. Infant Ment Health J 29, 203-218

Maercker A, Schützwohl M (1998): Erfassung von psychischen Belastungsfolgen: Die Impact of Event Skala-revidierte Version (IES-R). Diagnostica 44, 130-141

Malik M, Camm AJ (1993): Components of heart rate variability--what they really mean and what we really measure. Am J Cardiol $\underline{72}, 821-2$

Metzger LJ, Orr SP, Berry NJ, Ahern CE, Lasko NB, Pitman RK (1999): Physiologic reactivity to startling tones in women with posttraumatic stress disorder. J Abnorm Psychol 108, 347-352

Mezzacappa ES, Kelsey RM, Katkin ES, Sloan RP (2001): Vagal rebound and recovery from psychological stress. Psychosom Med 63, 650-7

Miller MW, Resick PA (2007): Internalizing and Externalizing Subtypes in Females Sexual Assault Survivors: Implications for the Understanding of Complex PTSD. Behav Ther 38, 58-71

Müller S, Sachsse U: Langfristige Chancen und Grenzen in der Traumatherapie. Beobachtungen zum Langzeitverlauf der komplexen posttraumatischen Belastungsstörung (kPTBS) In: Neurowissenschaften und Traumatherapie, hrsg. v. Jacobs S; Universitätsverlag, Göttingen 2009, 83-106

NICE (National Institute of Clinical Excellence) (2005): Posttraumatic Stress disorder - the management of PTSD in adult and children in primary and secondary care. National Clinical Practice guideline Number 26 http://www.nice.org.uk/guidance/

Orini M, Bailón R, Enk R, Koelsch S, Mainardi L, Laguna P (2010):A method for continuously assessing the autonomic response to music-induced emotions through HRV analysis. Med Biol Eng Comput 48, 423-33

Orr SP, Roth WT (2000): Psychophysiological assessment: clinical applications for PTSD. J Affect Disord 61, 225-240

Out D, Pieper S, Bakermans-Kranenburg MJ, van Ijzendoorn MH (2010): Physiological reactivity to infant crying: a behavioral genetic study. Genes Brain Behav $\underline{9}$, 868-76

Overkamp B: Differentialdiagnostik der dissoziativen Identitätsstörung (DIS) in Deutschland: Validierung der Dissociative Disorders Interview Schedule (DDIS). Med.Diss. München 2005

Overkamp B, Helmke-Jaeger S (2010): Dissoziative Störungen bei Patienten mit BorderlinePersönlichkeitsstörung und die Traumaätiologie. Persönlichkeitsstörungen - Theorie und Therapie (PTT) $\underline{14}, 117-125$

Panksepp J, Sacks DS, Crepeau LJ, Abbott BB: The psycho- and neurobiology of fear systems in the brain. In: Aversive Events and Behavior; hrsg v. Denny MR; Erlbaum, Hillsdale 1991, 7-59

Parati G, Di Rienzo M, Bertinieri G, Pomidossi G, Casadei R, Groppelli A, Pedotti A, Zanchetti A, Mancia G (1988): Evaluation of baroreceptor-heart rate reflex by 24-hour intra-arterial blood pressure monitoring in humans. Hypertension 12, 214-22

Peckerman A, Dahl K, Chemitiganti R, LaManca JJ, Ottenweller JE, Natelson BH (2003): Effects of posttraumatic stress disorder on cardiovascular stress responses in Gulf War veterans with fatiguing illness. Auton Neurosci 108, 63-72

Pelcovitz D, van der Kolk BA, Roth S, Mandel F, Kaplan S, Resick P (1997): Development of a criteria set and a structured interview for Disorders of Extreme Stress (SIDES). J Trauma Stress $\underline{10}, 3-16$

Penaz J, Voigt A, Teichmann W (1976): Contribution to the continous indirect blood pressure measurement. Z Gesamte Inn Med 31, 1030-1033 
Pole N (2007): The psychophysiology of posttraumatic stress disorder: a meta-analysis. Psychol Bull $133,725-746$

Reddemann L: Psychodynamisch imaginative Traumatherapie PITT: Das Manual. Pfeiffer, Stuttgart 2004

Reddemann L, Sachsse U (1997): Praxis der traumazentrierten Psychotherapie I: Stabilisierung. Persönlichkeitsstörungen - Theorie und Therapie (PTT) 1 , 113-147

Reddemann L, Sachsse, U (1998): Traumazentrierte Psychotherapie II: Traumaexposition. Persönlichkeitsstörungen - Theorie und Therapie (PTT) 2, 72-108

Richter P, Werner J, Heerlein A, Kraus A, Sauer H (1998): On the validity of the Beck-DepressionInventory. A review. Psychopathology $\underline{31}, 160-8$

Riem MM, Pieper S, Out D, Bakermans-Kranenburg MJ, van Ijzendoorn MH (2011): Oxytocin receptor gene and depressive symptoms associated with physiological reactivity to infant crying. Soc Cogn Affect Neurosci $\underline{6}, 294-300$

Ross CA, Heber S, Norton RG, Anderson D, Anderson G, Barchet P (1989): The Dissociative Disorder Interview Schedule: A Structured Interview. Dissociation 2, 169-189

Ross CA, Miller SD, Bjornson L, Reagor P, Fraser GA, Anderson G (1990): Structured Interview Data on 102 Cases of Multiple Personality Disorder from four Centers. Am J Psychiatry 147, 596601

Sachsse U (2002): Die posttraumatische Belastungsstörung: neurobiologisches Wissen und therapeutische Perspektiven. WsFPP $\underline{9}$, 79-100

Sachsse U (2003): Distress-Systeme des Menschen. Persönlichkeitsstörungen - Theorie und Therapie (PTT) $\underline{7}, 4-15$

Sachsse U, Eßlinger K, Schilling L (1997): Vom Kindheitstrauma zur schweren Persönlichkeitsstörung. Fund Psych 11, 12-20

Sachsse U, Vogel C, Leichsenring F (2006): Results of psychodynamically orientiented traumafocused inpatient treatment for women with complex posttraumatic stress disorder (PTSD) and borderline personality disorder (BPD). Bull Menninger Clinic 므, 125-144

Sachsse U, Weniger G, Lange C, Irle E: Neurobiologische Veränderungen nach Straftaten und anderen Traumatisierungen. In: Neurobiologie forensisch-relevanter Störungen. Grundlagen, Störungsbilder, Perspektiven; Müller J; Kohlhammer, Stuttgart 2009, 153-163

Sack M (2004): Diagnostische und klinische Aspekte der komplexen posttraumatischen Belastungsstörung. Nervenarzt $\underline{75}, 451-459$

Sack M, Hofmann A (2001): Interview zur komplexen posttraumatischen Belastungsstörung (IkPTBS), unveröffentlicht. Hannover

Sack M, Hopper JW, Lamprecht F (2004): Low respiratory sinus arrhythmia and prolonged psychophysiological arousal in posttraumatic stress disorder: heart rate dynamics and individual differences in arousal regulation. Biol Psychiatry 55, 284-290

Sack M, Sachsse U, Overkamp B, Dulz B (2012): Traumafolgestörungen bei Patienten mit BorderlinePersönlichkeitsstörung - Ergebnisse einer Multicenterstudie. (Nervenarzt, im Druck)

Sahar T, Shalev AY, Porges SW (2001): Vagal modulation of responses to mental challenge in posttraumatic stress disorder. Biol Psychiatry 49, 637-43

Schlögl A, Flotzinger D, Pfurtscheller G (1997): Adaptive autoregressive modeling used for singletrial EEG classification. Biomed Tech $\underline{42}, 162-167$

Schmahl CG, Vermetten E, Elzinga BM, Bremner JD (2003): Magnetic resonance imaging of hippocampal and amygdala volume in women with childhood abuse and borderline personality disorder. Psychiatry Res Neuroimag 122, 193-198.

Schmahl CG, Elzinga BM, Ebner UW, Simms T, Sanislow C, Vermetten E, McGlashan TH, Bremner JD (2004): Psychophysiological reactivity to traumatic and abandonment scripts in borderline personality and posttraumatic stress disorders: a preliminary report. Psychiatry Res $\underline{126}, 33-42$

Selye H: Einführung in die Lehre vom Adaptationssyndrom. Thieme, Stuttgart 1953 
Shaikh al arab A, Guédon-Moreau L, Ducrocq F, Molenda S, Duhem S, Salleron J, Chaudieu I, Bert D, Libersa C, Vaiva G (2012):Temporal analysis of heart rate variability as a predictor of post traumatic stress disorder in road traffic accidents survivors. J Psychiatr Res $\underline{46}, 790-6$

Shalev AY, Orr SP, Peri T, Schreiber S, Pitman RK (1992): Physiologic responses to loud tones in Israeli patients with posttraumatic stress disorder. Arch Gen Psychiatry $\underline{49}, 870-875$

Shapiro F: Eye movement desensitization and reprocessing: Basic principles, protocols and procedures. Guilford, New York 1995

Sloan RP, Shapiro PA, Bagiella E, Bigger JT Jr, Lo ES, Gorman JM (1996): Relationships between circulating catecholamines and low frequency heart period variability as indices of cardiac sympathetic activity during mental stress. Psychosom Med 58, 25-31

Spitzer C, Effler K, Freyberger H (2000): Posttraumatische Belastungsstörung, Dissoziation und selbstverletzendes Verhalten bei Borderline-Patienten. Z Psychosom Med Psychother 4ㅌ, $273-$ 285

Stein MB, Yehuda R, Koverola C, Hanna C (1997): Enhanced dexamethasone suppression of plasma cortisol in adult women traumatized by childhood sexual abuse. Biol Psychiatry $\underline{42}, 680-6$

Tan G, Dao TK, Farmer L, Sutherland RJ, Gevirtz R (2011): Heart rate variability (HRV) and posttraumatic stress disorder (PTSD): a pilot study. Appl Psychophysiol Biofeedback $\underline{36}, 27-$ 35

Task Force of the European Society of Cardiology and the North American Society of Pacing and Electrophysiology (1996): Heart rate variability. Standards of measurement, physiological interpretation, and clinical use. Eur Heart J $\underline{17}, 354-81$

Thayer JF, Lane RD (2000): A model of neurovisceral integration in emotion regulation and dysregulation. J Affect Disor 61, 201-216

Tkaczyszyn M, Olbrycht T, Makowska A, Soboń K, Paleczny B, Rydlewska A, Jankowska EA (2012): The influence of the sounds of crying baby and the sounds of violence on haemodynamic parameters and autonomic status in young, healthy adults. Int J Psychophysiol $\underline{87}, 52-9$

Tsuji H, Larson MG, Venditti FJ Jr, Manders ES, Evans JC, Feldman CL, Levy D (1996): Impact of reduced heart rate variability on risk for cardiac events. The Framingham Heart Study. Circulation 94, 2850-5

van der Zwaag MD, Westerink J, van den Broek E (2011): Emotional and psychophysiological responses to tempo, mode, and percussiveness. Music Sci $\underline{15}$, 250-269

van Ijzendoorn MH, Schuengel C (1996): The measurement of dissociation in normal and clinical populations: Meta-analytic validation of the Dissociative Experiences Scale (DES). Clin Psychol Rev 16, 365-382

Walczewska J, Rutkowski K, Wizner B, Cwynar M, Grodzicki T (2011): Stiffness of large arteries and cardiovascular risk in patients with post-traumatic stress disorder. Eur Heart J $\underline{32}, 730-6$

Weiss DS, Marmar CR: The Impact of Event Scale - Revised. In: Assessing psychological trauma and PTSD; Wilson JP, Keane TM; Guilford, New York 1996, 399-411

WHO - Weltgesundheitsorganisation, Dilling H, Mombour W, Schmidt MH: Internationale Klassifikation psychischer Störungen. ICD-10 Kapitel V (F). Klinisch diagnostische Leitlinien. Hans Huber Verlag, Bern-Göttingen-Toronto 2006

Weniger G, Lange C, Sachsse U, Irle E (2008): Amygdala and hippocampal volumes and cognition in adult survivors of childhood abuse with dissociative disorders. Acta Psychiatr Scand 118, 281290

Wöller W; Kruse J (2003): Persönlichkeitsstörungen und die Psychopathologie in der Folge von Traumen. Überlegungen zur diagnostischen Klassifikation. Nervenarzt 74, 972-976

Wolpe, J: Psychotherapy by Reciprocal Inhibition. Stanford University Press, Stanford 1958

Yehuda R (2002): Current status of cortisol findings in post-traumatic stress disorder. Psychiatr Clin North Am 25, 341-68 
Yehuda R, Kahana B, Binder-Brynes K, Southwick SM, Mason JW, Giller EL (1995): Low urinary cortisol excretion in Holocaust survivors with posttraumatic stress disorder. Am J Psychiatry $152,982-6$

Zanarini MC, Williams AA, Lewis RE, Reich RB, Vera SC, Marino MF, Levin A, Yong L, Frankenburg FR (1997): Reported pathological childhood experiences associated with the development of borderline personality disorder. Am J Psychiatry 154, 1101-6

Zanarini MC, Frankenburg FC, Hennen J, Reich DB, Silk KR (2006): Prediction of the 10-year course of borderline personality disorder. Am J Psychiatry 163, 827-832

Zucker M, Spinazzola J, Blaustein M, Van der Kolk B (2006): Dissociative symptomatology in posttraumatic stress disorder and disorders of extreme stress. J Trauma Dissociation $\underline{7}, 19-31$ 


\section{Abbildungsverzeichnis}

Abbildung 1: Hauptsymptomatik der posttraumatischen Belastungsstörung .................................. 7

Abbildung 2: Studienablauf: einzelne Untersuchungsabschnitte für Patientinnen .............................22

Abbildung 3: Messprotokoll der Stressbelastungsuntersuchung .....................................................28

Abbildung 4: Hämodynamische und autonome Task-Force-Monitor-Parameter zur Beurteilung der

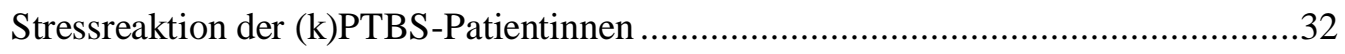

Abbildung 5: Kriterien für die Zugehörigkeit zur Subgruppe „hoch Borderline“. „Hoch Borderline“ wird angenommen, wenn alle drei genannten Kriterien erfüllt sind.

Abbildung 6: Vorhandene Datensätze [n] für die Teiluntersuchungen der Studie (Diagnostische Interviews, Fragebögen, TFM-Messung, Analogskala zum subjektiven

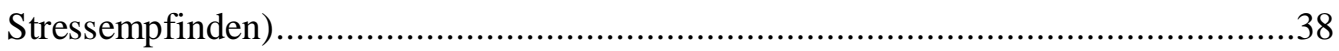

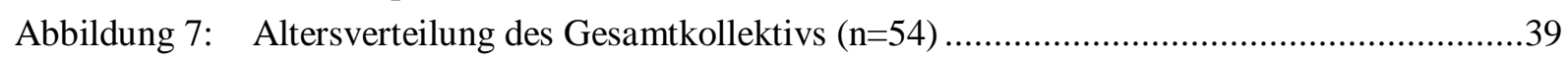

Abbildung 8: Häufigkeitsverteilung (Anzahl und Prozente) der traumaassoziierten Störungsbilder im

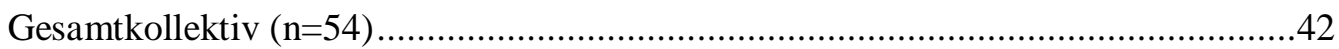

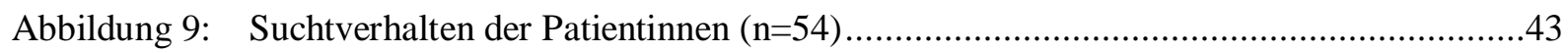

Abbildung 10: Angaben des subjektiven Stressempfindens (Analogskala: $0=$,gar kein Stress“ bis 10 =,,schlimmster Stress“) während der Stressbelastungsuntersuchung - Verteilung als Whisker-Boxplot (Median, Quartile, Minimum, Maximum) für die einzelnen Messphasen. 48

Abbildung 11: Häufigkeitsverteilung des subjektiven Stressempfindens (Analogskala 0-10) während des Kopfrechentests im Gesamtkollektiv ( $\mathrm{n}=54)$

Abbildung 12: Häufigkeitsverteilung des subjektiven Stressempfindens (Analogskala 0-10) während des Babyschreiens im Gesamtkollektiv ( $\mathrm{n}=54)$

Abbildung 13: Reaktionsformen während der Stressbelastungsuntersuchung (Rechnen, Babyschreien und Entspannungsmusik).

Abbildung 14: Patientinnen-Bewertung der Entspannungsmusik $(\mathrm{n}=54)$........................................52

Abbildung 15: Abbruchquote im Gesamtkollektiv ( $n=54)$ für die einzelnen Stresstests während der Stressbelastungstests (Kopfrechnen, Babyschreien, Entspannungsmusik)..................52

Abbildung 16: Verlauf des Cardiac Index (CI) während der Stressbelastungsuntersuchung, Phasenmittelwerte und 95\%-Konfidenzintervalle ...................................................56

Abbildung 17: Verlauf der Herzfrequenz (HR) während der Stressbelastungsuntersuchung, Phasenmittelwerte und 95\%-Konfidenzintervalle.................................................57

Abbildung 18: Verlauf des Totalen peripheren Widerstands-Index (TPRI) während der Stressbelastungsuntersuchung, Phasenmittelwerte und 95\%-Konfidenzintervalle .......57

Abbildung 19: Verlauf des systolischen, diastolischen und mittleren Blutdrucks (sBP, dBP, mBP) während der Stressbelastungsuntersuchung, Phasenmittelwerte und 95\%-

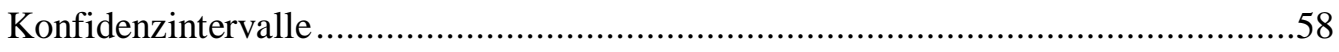

Abbildung 20: Verlauf der Standardabweichung aller RR-Intervalle (SDNN) während der Stressbelastungsuntersuchung, Phasenmittelwerte und 95\%-Konfidenzintervalle .......59

Abbildung 21: Verlauf der HF-HRV (logarithmiert) während der Stressbelastungsuntersuchung, Phasenmittelwerte und 95\%-Konfidenzintervalle. 60 
Abbildung 22: Verlauf der LF-HRV (logarithmiert) während der Stressbelastungsuntersuchung, Phasenmittelwerte und 95\%-Konfidenzintervalle . .61

Abbildung 23: Verlauf der Barorezeptorsensitivität (Slope Mean Total) während der Stressbelastungsuntersuchung, Phasenmittelwerte und 95\%-Konfidenzintervalle ......61

Abbildung 24: Verhalten der TFM-Parameter während der beiden Stresstests „Rechnen“ und „Babyschreien“ in Bezug zur Ruhephase 1 (Baseline), Signifikanzen des Phasenvergleiches Ruhe 1 vs. Stresstest sowie Rechnen vs. Babyschreien: * $\mathrm{p}<0,05$, $* * \mathrm{p}<0,01, * * \mathrm{p}<0,001$ (adjustiert)

Abbildung 25: Paarvergleich Rechnen vs. Babyschreien: Verhalten der TFM-Parameter in der Subgruppe "hoch Borderline“ $[n=16]$ und in der Vergleichsgruppe [n=30], Signifikanzen des Gruppenvergleiches der Phasenmittelwert-Differenzen $* p<0,05$, $* * \mathrm{p}<0,01, * * * \mathrm{p}<0,001$

Abbildung 26: Verlauf systolischer arterieller Blutdruck (sBP) von Subgruppe „hoch Borderline“ und Vergleichsgruppe während der Stressbelastungsuntersuchung, Phasenmittelwerte und 95\%-Konfidenzintervalle. . .70

Abbildung 27: Verlauf Barorezeptorsensitivität (Slope Mean Total) von Subgruppe „hoch Borderline" und Vergleichsgruppe während der Stressbelastungsuntersuchung, Phasenmittelwerte und 95\%-Konfidenzintervalle 


\section{Tabellenverzeichnis}

Tabelle 1: $\quad$ Kriterien der kPTBS nach Herman 1992

Tabelle 2: Diagnostische Kriterien der Borderline-Persönlichkeitsstörung nach DSM-IV (APA 2003)

Tabelle 3: $\quad$ Soziodemographische Daten des Gesamtkollektivs zum Zeitpunkt der Messung .......40

Tabelle 4: $\quad$ Risikoprofil des Gesamtkollektivs zum Zeitpunkt der Messung ................................41

Tabelle 5: $\quad$ Verteilung der Begleitdiagnosen im Gesamtkollektiv ...............................................43

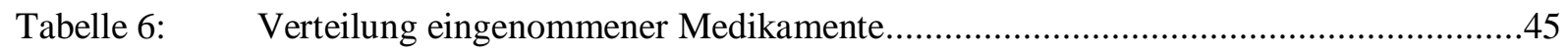

Tabelle 7: Psychometrische Befunde der Patientinnen...................................................................

Tabelle 8: $\quad$ Subjektives Stressempfinden (Analogskala) während der

Stressbelastungsuntersuchung (Rechnen, Babyschreien, Entspannungsmusik)

Tabelle 9: Beobachtbare emotionale bzw. vegetative Reaktionen während der

Stressbelastungstests

Tabelle 10: Korrelation nach Kendall-Tau-b zwischen der Anzahl der beobachteten vegetativen Reaktionen und TFM-Parametern während des Babyschreiens, Korrelationskoeffizient und ggf. Signifikanzniveau $\left({ }^{*} \mathrm{p}<0,05, * * \mathrm{p}<0,01\right)$

Tabelle 11: Hämodynamische (CI, HR, TPRI, sBP, dBP, mBP) und autonome (SDNN, HF-HRV, LF-HRV, BRS) TFM-Parameter im Verlauf der Stressbelastungsuntersuchung; Mittelwerte \pm Standardabweichung, Anzahl [n], ggf. p-Wert der globalen ANOVA...54

Tabelle 12: Sechsfacher Paarvergleich (Ruhe 1 vs. Rechnen/ Ruhe 2/ Babyschreien/ Musik, Rechnen vs. Babyschreien) der in der globalen ANOVA signifikanten TFM-Parameter (CI, HR, TPRI, sBP, dBP, mBP, SDNN, LF-HRV, BRS) für das Gesamtkollektiv [ $n=52]$, Phasenmittelwert-Differenzen \pm Standardfehler sowie ggf. $p$-Wert.

Tabelle 13: $\quad$ Ausgewählte TFM-Parameter (CI, sBP, TPRI, RMSSD) zur Beurteilung der Erholung nach Babyschreien für vier Messzeitpunkte: Mittelwerte von jeweils fünf Herzschlägen \pm Standardabweichung und Anzahl [n], ggf. p-Wert der ANOVA .......63

Tabelle 14: Psychometrischer Befund der Subgruppe "hoch Borderline" und Vergleichsgruppe ..65 Tabelle 15: Angaben des subjektiven Stressempfindens während der Stressbelastungsuntersuchung von Subgruppe "hoch Borderline" [n=16] und Vergleichsgruppe [n=30], Mittelwerte \pm Standardabweichung und ggf. $p$-Wert.

Tabelle 16: Anzahl beobachteter vegetativer Reaktionen während des Babyschreiens von Subgruppe "hoch Borderline" [ $n=16]$ und Vergleichsgruppe [n=30], Häufigkeiten und Prozente

Tabelle 17: Korrelation nach Kendall-Tau-b zwischen den beobachteten vegetativen Reaktionen und den TFM-Parametern während des Babyschreiens für die Subgruppe "hoch Borderline" [n=16] und die Vergleichsgruppe [n=30], Korrelationskoeffizient und ggf. Signifikanzniveau $(* \mathrm{p}<0,05, * * \mathrm{p}<0,01)$

Tabelle 18: $\quad$ Paarvergleich der Stressoren (Rechnen vs. Babyschreien) für die TFM-Parameter HR, CI, TPRI, sBP, HF-HRV, LF-HRV, BRS in der Subgruppe "hoch Borderline" [n=16] und der Vergleichsgruppe [n=30], Phasenmittelwert-Differenzen \pm Standardfehler sowie ggf. p-Wert. 


\section{Anhang}

\subsection{Patientinnenaufklärung}

\section{UNIVERSITÄTSMEDIZIN :
GÖTTINGEN $=1 / 0$}

Universitätsmedizin Göttingen, 37099 Göttingen

Psychosomatische Medizin und Psychotherapie, von-Siebold-Str. 5, 37075 Göttingen
Zentrum Psychosoziale Medizin Psychosomatische Medizin und Psychotherapie Direktor: Prof. Dr. med. Christoph Herrmann-Lingen

Ärztiche Leltung

37099 Göttingen Briefpos 37075 Göttingen Adress 0551/39-6707 Telefon 0551/39-19150 Fax

E-Mail

Aktenzeichen 12. März 2013 Datum

Patientinnenaufklårung

Klinische Studie:

Physiologische Stressreagibilität bei Frauen mit komplexer posttraumatischer Belastungsstörung bzw. Borderline-Persönlichkeitsstörung vor und nach psychotherapeutischer Behandlung sowie im Vergleich zu gesunden Frauen

Sehr geehrte Patientin,

im Rahmen einer wissenschaftlichen Studie möchten wir Sie bitten und einladen, sich an einer freiwilligen Untersuchung zu beteiligen. Wir wollen mögliche Zusammenhänge zwischen HerzKreislauf-Funktion und einer komplexen posttraumatischen Belastungsstörung bzw. BorderlinePersönlichkeitsstörung ermitteln. Eine Frage ist dabei, wie das Herz-Kreislauf-System in Ruhe/Entspannung, aber auch auf alltägliche Stressbelastungen reagiert. Von den Studienergebnissen erhoffen wir uns ein besseres Verständnis der Zusammenhänge von psychischer Befindlichkeit einerseits und einer veränderten Herz-Kreislauf-Funktion andererseits.

Wenn Sie der Studienteilnahme zustimmen, würden wir eine EKG-Ableitung und eine Messung von Blutdruck und Herzschlag durchführen. Während der Messung sind 2 kurze Tests zur Simulation alltäglicher Stressbelastung sowie eine Entspannungsphase geplant. Die Untersuchung dauert insgesamt ca. 2 Stunden. Ergebnisse der Untersuchungen teilen wir Ihnen auf Wunsch gern mit. Zusätzlich würden wir einige Befunde aus Ihrer Krankenakte bei der Auswertung verwenden. Prof. Sachsse wird diesbezüglich von seiner ärztlichen Schweigepflicht entbunden. 


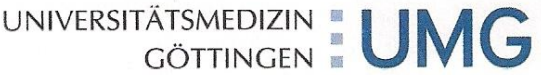

Ihre Studienteilnahme hat keinerlei Einfluss auf Ihre medizinische Behandlung, auch entsteht für Sie durch die Studienteilnahme kein unmittelbarer Nutzen aber auch kein zusätzliches Risiko, zumal keine Blutentnahme und keine zusätzliche Medikamentengabe stattfinden.

Die für die Studie erhobenen bzw. Ihrer Krankenakte entnommenen Befunde werden unter strenger Beachtung der Schweigepflicht nur in pseudonymisierter Form verarbeitet und sind Dritten nicht zugänglich.

Für Fragen stehen wir Ihnen persönlich oder telefonisch gern zur Verfügung.

Mit freundlichen Grüßen,

\section{Prof. Dr. Ch. Herrmann-Lingen}

Universitätsmedizin Göttingen

Abt. Psychosomatische Medizin und Psychotherapie von Siebold-Str. 5, 37075 Göttingen

Tel. 0551 - 396707

\section{Prof. Dr. U. Sachsse}

Asklepios Fachklinikum Göttingen Abteilung Psychotherapie Rosdorfer Weg 70, 37081 Göttingen Tel. 0551 - 4021010 


\subsection{Patientinneneinwilligung}

\section{UNIVERSITÄTSMEDIZIN $=1 / 9$
GÖTTINGEN $=$}

Universitätsmedizin Göttingen, 37099 Göttingen

(1)
Zentrum Psychosoziale Medizin Psychosomatische Medizin und Psychotherapie Direktor: Prof. Dr. med. Christoph Herrmann-Lingen

Årztliche Leitung

37099 Gö̈ttingen Briefpost von Siebold-Str. 5, 37075 Göttingen Adresse 0551 / 39-6707 Telefon $0551 / 39-19150$ Fax E-Mail

Aktenzeichen 12. März 2013 Datum

(Patientinnenaufkleber bzw. Probandinnencode)

\section{Einwilligungserklårung}

Klinische Studie: Physiologische Stressreagibilitüt bei Frauen mit komplexer posttraumatischer Belastungsstörung brw. Borderline-Persönlichkeitsstörung vor und mach psychotherapeutischer Behandlung sowie im Vergleich zu gesunden Frauen

Hiermit erkläre ich mein Einverståndnis mit der Teilnahme an der oben genannten klinischen Studie zur Bedeutung des Einflusses einer komplexen posttraumatischen Belastungsstörung bzw. Borderline-Persönlichkeitsstörung auf die Herz-Kreislauf-Funktion.

Ich wurde über den Inhalt der Studie und die geplanten Maßnahmen informiert. Alle meine Fragen im Zusammenhang mit der Studie wurden hinreichend beantwortet.

Mir ist bekannt, dass ich meine Einwilligung jederzeit ohne Angaben von Gründen widerrufen kann, ohne dass mir daraus irgendwelche Nachteile entstehen.

Bei dieser Studie werden die Vorschriften über die ärztliche Schweigepflicht und den Datenschutz eingehalten. Es werden persönliche Daten und Befunde über Sie erhoben, gespeichert und verschlüsselt (pseudonymisiert, d.h. weder Ihr Name noch Ihre Initialen oder das exakte Geburtsdatum erscheinen im Verschlüsselungscode) weitergegeben.

Im Falle Ihres Widerrufs der Einwilligung werden die pseudonymisiert gespeicherten Daten vernichtet. 


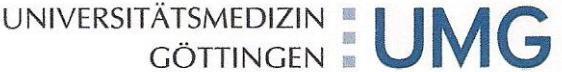

Der Zugang zu den Originaldaten und zum Verschlüsselungscode ist auf folgende Personen beschränkt: Studienleiter: Prof. Dr. Ch. Herrmann-Lingen, Prof. Dr. U. Sachsse sowie im Projekt tätige Doktorandinnen. Die Unterlagen werden in der Abteilung Psychosomatische Medizin und Psychotherapie der Universität Göttingen unter Verschluss aufbewahrt.

Im Falle von Veröffentlichungen der Studienergebnisse bleibt die Vertraulichkeit der persönlichen Daten ebenfalls gewährleistet.

Ich möchte über die Ergebnisse dieser Untersuchung in Kenntnis gesetzt werden.

ja

nein

Göttingen, den

(Unterschrift der Patientin bzw. Probandin)

Göttingen, den

(Unterschrift der aufklärenden Versuchsleiterin/des aufklärenden Versuchsleiters)

Ansprechpartner für Rückfragen:

Prof. Dr. Ch. Herrmann-Lingen

Universitätsmedizin Göttingen

Abt. Psychosomatische Medizin und Psychotherapie

von Siebold-Str. 5, 37075 Göttingen

Tel. $0551-396707$
Prof. Dr. U. Sachsse

Asklepios Fachklinikum Göttingen

Abteilung Psychotherapie

Rosdorfer Weg 70, 37081 Göttingen

Tel. $0551-40210$ 


\subsection{Allgemeiner Fragebogen zur Erhebung soziodemographischer Daten}

\section{UNIVERSITÄTSMEDIZIN $=\mathbb{N}$
GÖTTINGEN}

Studie zur physiologischen Stressreagibilität bei Frauen mit komplexer posttraumatischer Belastungsstörung bzw. Borderline-Persönlichkeitsstörung vor und nach psychotherapeutischer Behandlung sowie im Vergleich zu gesunden Frauen

\section{Allgemeiner Fragebogen}

1) Wie alt sind Sie?

2) Wie groß sind Sie?

3) Wieviel wiegen Sie?

4) Befinden Sie sich in einer festen Partnerschaft?

Wenn ja:

Leben Sie mit Ihrem Partner / Ihrer Partnerin zusammen? Sind sie verheiratet?

5) Sind Sie geschieden?

6) Sind Sie verwitwet?

7) Haben Sie Kinder?

Wenn ja: Welches Alter haben Ihre Kinder?

8) Treiben Sie regelmäßig Sport?
Wieviele Stunden pro Woche?
$O \leq 1 / 2 h$
$\mathrm{O} 1 / 2-2 \mathrm{~h}$
3-5h

Code: Jahre $\mathrm{cm}$ $\mathrm{kg}$

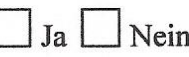

$\square_{\text {Ja }} \square$ Nein $\square_{\text {Ja }} \square$ Nein $\square$ Ja $\square$ Nein $\square$ Ja $\square$ Nein $\square$ Ja $\square$ Nein Jahre

9) Wieviel Alkohol trinken Sie pro Woche?

$$
\begin{aligned}
& \text { (Wird vom Untersucher ausgefüllt: } \\
& (0,251 \mathrm{Bier} / 0,11 \text { Wein/ } 2 \mathrm{cl} \text { Schnaps = } 1 \text { Drink) }
\end{aligned}
$$

entspricht dpw)

10) Rauchen Sie?

Seit wann?

Wenn ja:

Wieviele Packungen pro Tag?

(1 Packung $\cong 20$ Zigaretten)

(Wird vom Untersucher ausgefüllt:

$($ Packyears $=$ tägl. konsumierte Zigarettenpackungen $\mathrm{x}$ Jahre Zigarettenkonsum $)$ 


\section{UNIVERSITÄTSMEDIZIN $=\square$
GÖTTINGEN $=0$}

11) Welchen höchsten allgemeinbildenden Schulabschluss haben Sie?

O ohne Schulabschluss

O Hauptschule / Volksschule

O Realschule (Mittlere Reife)

O Polytechnische Oberschule

O Fachhochschule

O Hochschulreife / Abitur (Gymnasium oder EOS)

$O$ anderer Schulabschluss:

12) Welchen beruflichen Ausbildungsabschluss haben Sie?

(Bitte alle zutreffenden Antworten ankreuzen.)

ich habe keinen Berufsabschluss und befinde mich nicht in einer Ausbildung

$O$ ich befinde mich noch in meiner Ausbildung

$O$ beruflich-betriebliche Ausbildung (Lehre)

O beruflich-schulische Ausbildung (Berufsfach- / Handelsschule)

O Fach- / Meister- / Technikerschule / Berufs- / Fachakademie

O Fachhochschule

O Hochschule

$O$ anderer Berufsabschluss:

13) Sind Sie zur Zeit erwerbstätig?

Wenn ja: Wieviele Stunden / Woche?

O Vollzeit $\geq 35 \mathrm{~h} /$ Woche

O Teilzeit $15 \ldots<35 \mathrm{~h} /$ Woche

$\bigcirc$ Teilzeit (auch stundenweise) $<15 \mathrm{~h} /$ Woche

14) Nehmen Sie Medikamente? Welche?

(z.B. Pille, Herzmittel,...)

15) Haben Sie Vorerkrankungen?

(z.B. Bluthochdruck, Diabetes, ...)

16) Fühlen Sie sich in letzter Zeit besonders gestresst? 


\section{Danksagung:}

An dieser Stelle möchte ich die Gelegenheit nutzen, all denen zu danken, die die vorliegende Studie ermöglicht und mich beim Verfassen meiner Doktorarbeit unterstützt haben.

Ich danke Prof. Herrmann-Lingen für die freundliche Überlassung des hochinteressanten Themas und seine fachliche Betreuung mit konstruktiver Kritik während des gesamten Entstehungsprozesses meiner Arbeit.

Prof. Sachsse möchte ich herzlich für die Hilfe bei der Rekrutierung der Patientinnen und seine wertvollen inhaltlichen Erläuterungen zum Themenkomplex der Traumafolgestörungen danken, die mich auf den Umgang mit dieser vulnerablen Patientengruppe gut vorbereitet und währenddessen begleitet haben.

Ich danke dem gesamten Team der Station 9 des AFG, die den organisatorischen Ablauf der Studie ermöglicht haben, sei es durch Vorbereitung und Gespräche mit den Patientinnen oder Datenerhebung im Rahmen der Diagnostik.

Ich danke den Mitarbeitern der medizinischen Statistik, insbesondere Herrn Simon Schneider, für die Hilfe bei der statistischen Auswertung der erhobenen Daten.

Ein besonderer Dank gilt meiner Mitdoktorandin Gesine Bornschein für die außerordentlich gute Zusammenarbeit.

Nicht zuletzt möchte ich meine Anerkennung und Dankbarkeit den Patientinnen und Probandinnen aussprechen, die sich bereit erklärt haben, an der Studie teilzunehmen, um den Forschungsstand zur Stressreagibilität bei komplexen Traumafolgestörungen voranzubringen. 


\section{Lebenslauf:}

Ich, Juliane Albrecht, wurde am 18.06.1985 als Tochter von Dr. Wolfgang Albrecht und Petra Albrecht, geb. Dietze, in Erfurt geboren. Von 1992 bis 1996 besuchte ich die Grundschule am Roten Berg in Erfurt. Die Gymnasialzeit absolvierte ich an der Edith-Stein-Schule in Erfurt, wo ich im Juni 2004 das Abitur ablegte. Anschließend war ich zehn Monate als Volontärin in einem Kinderheim in Greytown, Südafrika tätig und absolvierte mein Pflegepraktikum in einem Erfurter Krankenhaus. Im Oktober 2005 begann ich das Studium der Humanmedizin an der Georg-August-Universität in Göttingen und bestand dort im September 2007 das Physikum. Während des Studiums machte ich zahlreiche Praktika im In- und Ausland und war als studentische Hilfskraft im Studentischen Trainingszentrum Ärztlicher Praxis und Simulation der Universitätsmedizin Göttingen tätig. Das praktische Jahr absolvierte ich ab Februar 2011 in Durban, Südafrika (Chirurgie), Bremen (Innere Medizin) und Göttingen (Anästhesie), wo ich im April 2012 mein Studium erfolgreich mit der zweiten ärztlichen Prüfung beendete. Die Approbation als Ärztin erhielt ich im Juni 2012. Anschließend arbeitete ich an der 2009 begonnenen, hier vorliegenden klinischen Dissertation in der Klinik für Psychosomatische Medizin und Psychotherapie der UMG in Zusammenarbeit mit dem Asklepios Fachklinikum unter der Betreuung von Prof. Herrmann-Lingen und Prof. Sachsse. Seit Januar 2013 bin ich als Assistenzärztin in der zweiten Medizinischen Klinik des Helios Klinikums Erfurt tätig. 